\title{
Repair mechanisms in abdominal tissue
}

Citation for published version (APA):

Liu, H. (2021). Repair mechanisms in abdominal tissue: promotion of fascial healing and prevention of intra-abdominal adhesions. [Doctoral Thesis, Maastricht University]. ProefschriftMaken.

https://doi.org/10.26481/dis.20210518hl

Document status and date:

Published: 01/01/2021

DOI:

10.26481/dis.20210518hl

Document Version:

Publisher's PDF, also known as Version of record

\section{Please check the document version of this publication:}

- A submitted manuscript is the version of the article upon submission and before peer-review. There can be important differences between the submitted version and the official published version of record.

People interested in the research are advised to contact the author for the final version of the publication, or visit the DOI to the publisher's website.

- The final author version and the galley proof are versions of the publication after peer review.

- The final published version features the final layout of the paper including the volume, issue and page numbers.

Link to publication

\footnotetext{
General rights rights.

- You may freely distribute the URL identifying the publication in the public portal. please follow below link for the End User Agreement:

www.umlib.nl/taverne-license

Take down policy

If you believe that this document breaches copyright please contact us at:

repository@maastrichtuniversity.nl

providing details and we will investigate your claim.
}

Copyright and moral rights for the publications made accessible in the public portal are retained by the authors and/or other copyright owners and it is a condition of accessing publications that users recognise and abide by the legal requirements associated with these

- Users may download and print one copy of any publication from the public portal for the purpose of private study or research.

- You may not further distribute the material or use it for any profit-making activity or commercial gain

If the publication is distributed under the terms of Article $25 \mathrm{fa}$ of the Dutch Copyright Act, indicated by the "Taverne" license above, 


\section{Repair mechanisms in abdominal tissue}

Promotion of fascial healing and prevention of intra-abdominal adhesions 
Copyright (C) Hong Liu 2021

All rights reserved. No part of this thesis may be reproduced or distributed in any form or by any means, without the prior written permission of the author or the publisher.

Cover: Hong Liu

Layout: Tiny Wouters

Printing: ProefschriftMaken

ISBN: 978-94-6423-203-5

Printing of this thesis was financially supported by Maastricht University, 


\title{
Repair mechanisms in abdominal tissue
}

Promotion of fascial healing and prevention of intra-abdominal adhesions

\author{
DISSERTATION \\ To obtain the double doctoral degree at Maastricht University, the Netherlands \\ on the authority of Rector Magnificicus prof. dr. Rianne M. Letschert \\ in accordance with the decision of the Board of Deans, \\ to be defended in public on \\ Tuesday May $18^{\text {th }} 2021$ at 16:00 hours
}

by

Hong Liu 


\section{Promotor}

Prof. dr. N.D. Bouvy

\section{Copromotor}

Dr. K. Lenaerts

\section{Beoordelingscommissie}

Prof. dr. L.P.S Stassen, voorzitter

Prof. dr. R. Van der Hulst

Prof. dr. J. Lange

Prof. dr. L. Moroni

Dr. R.P.G. ten Broek 


\section{Table of contents}

$\begin{array}{lll}\text { Chapter } 1 \text { General introduction and outline of the thesis } & 7\end{array}$

$\begin{array}{lll}\text { Part I } & 19\end{array}$

Chapter 2 Do ARRIVE guidelines improve the quality of mesh repair 21 experiments in animals?

Chapter 3 Baseline performance of the ischaemic button model for 55 induction of adhesions in laboratory rats

Part II Repair of fascia in the abdominal wall $\quad 71$

$\begin{array}{lll}\text { Chapter } 4 & \text { Temporal changes in cell populations and gene expression } & 73\end{array}$ during abdominal wall fascial healing

Chapter 5 Abdominal fascial healing in the first postoperative week: a MALDI-TOF mass spectrometry imaging to investigate lipids in rats

$\begin{array}{ll}\text { Part III Too much repaired tissue in case of adhesions } & 111\end{array}$

Chapter 6 Comparing five new polymer barriers for the prevention of 113 intra-abdominal adhesions in a rat model

Chapter 7 Prevention of intra-abdominal adhesions by a hyaluronic acid gel; 127 an experimental study in rats

Chapter 8 Comparison of coated meshes for intraperitoneal placement in animal studies: a systematic review and meta-analysis

$\begin{array}{ll}\text { Part IV Discussion and summary } & 181\end{array}$

$\begin{array}{lll}\text { Chapter } 9 & \text { General discussion and future perspectives } & 183\end{array}$

$\begin{array}{lll}\text { Chapter } 10 & \text { Summary } & 197\end{array}$

第十章 中文总结 203

$\begin{array}{lll}\text { Chapter } 11 & \text { Valorisation } & 207\end{array}$

$\begin{array}{llr}\text { Part V Appendix } & 215\end{array}$

$\begin{array}{ll}\text { Acknowledgements } & 217\end{array}$

$\begin{array}{ll}\text { List of publications } & 223\end{array}$

$\begin{array}{ll}\text { Curriculum Vitae } & 227\end{array}$ 



\section{Chapter 1}

General introduction and outline of the thesis 



\section{Introduction}

In 1807, the first elective laparotomy with satisfying recovery was performed successfully. ${ }^{1}$ Currently, more than 20,000 major abdominal surgeries are performed annually in the Netherlands. ${ }^{2,3}$ Incisional hernias $(\mathrm{IH})^{4}$ and intra-abdominal adhesions ${ }^{5,6}$ are two of the most common complications following abdominal surgeries; these complications are related to abnormal healing processes. Generally, the abdominal healing process after surgical injuries involves regeneration or repair of damaged tissues. The term 'regeneration' is usually defined as new growth of tissues completely restoring the structure and function of the damaged tissues to their original state. ${ }^{7}$ Tissue repair may restore part of the structure and function of the damaged tissues but may form abnormal structures that impair the normal function of the original tissues. ${ }^{7}$ Optimal fascial healing in abdominal wall is essential to reduce the formation of $\mathrm{IH}^{8}{ }^{8}$ while intraperitoneal adhesions are linked to over-restored fibrous tissues.

\section{Postoperative fascial healing}

The fascia is a layer of fibrous connective tissue distributing ubiquitously in different locations of the body; it covers virtually every structure within the body, providing every organ with structural support and function. ${ }^{9}$ Abdominal fascial continuity plays an important role in preventing abdominal $\mathrm{IH}$, a protrusion of internal organs through a surgical scar defect in the abdominal wall ${ }^{10}$ (Figure 1.1). IH remains one of the most frequent complications in visceral surgery especially following a midline incision in the abdominal wall. The incidence of IH after laparotomy is around $13 \%$ at two postoperative years and numbers can increase with longer follow-up. ${ }^{11,12}$ The related costs for Europe are billions of Euros every year. ${ }^{13,14}$ It is considered that early mechanical failure of fascial healing, via aberrant fibroblast function, leads to the formation of $\mathrm{IH}^{15,16}$

Several risk factors for IH development have been identified, including obesity, diabetes, abdominal aortic aneurysm, current smoking, male sex, increasing age, previous laparotomy, and co-morbidities increasing intra-abdominal pressure. ${ }^{11,18}$ Identifying patients who are at risk to develop IH will help surgeons with their decision process and will provide strategies preventing IH. A surgery-specific decision-support interface tool has been developed to predict high-risk IH patients. ${ }^{19}$ Moreover, an electronic nose measuring volatile organic compounds in expiration can distinguish patients at risk of hernia recurrence from healthy controls. ${ }^{20}$ 

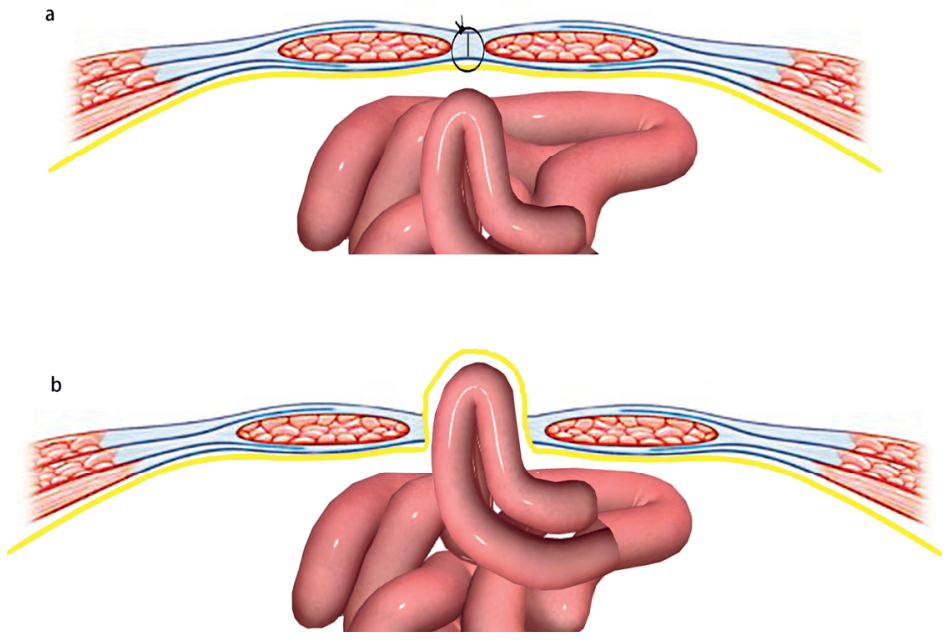

Figure 1.1 In (a), a transverse section from above arcuate line in abdominal wall is presented. Midline incision of fascia is closed by chronic absorbable suture and healed normally. In (b), a loop of small intestine covered with intact peritoneum protrudes through a defect of fascia in abdominal wall, forming an IH.

Note. This illustration is a modification from a picture of Bingöl et al. (2016). ${ }^{17}$

Generally, there are four stages in wound healing, including hemostasis, inflammation, proliferation, and remodelling. Despite the fact that normal healing of virtually all acutely injured soft tissues passes through similar stages, the underlying molecular events differ between specific tissues. ${ }^{21}$ Abdominal fascial healing is better studied as a separate entity rather than being studied within the context of skin wound healing. Evidence has shown that fascial incisions differ from skin wounds concerning the rate of the healing process. Controversy exists regarding whether fascia heals faster or skin heals faster. European Hernia Society guidelines recommend using slowly absorbable suture rather than rapidly absorbable suture in a single layer aponeurotic closure technique for abdominal wall fascial closure. ${ }^{22}$ This recommendation is mainly based on the evidence that the use of a slowly absorbable suture significantly reduces the incidence of IH compared to the use of a rapidly absorbable suture. ${ }^{22}$ For instance, polydioxanone suture, a recommended slowly absorbable suture commonly used for fascia closure, can remain up to 42 days for wound support despite the gradual decrease in tensile strength during the postoperative period. In contrast, the suture supporting skin wound closure can be removed in only one to two weeks. However, Franz et al. and Dubay et al. have reported that fascia heals faster than skin with respect to the 
regaining of mechanical strength, and has greater fibroblast activity. ${ }^{23,24}$ In contrast, Douglas has reported that skin requires six weeks while fascia needs at least 14 weeks to regain $60 \%$ of its unwounded strength. ${ }^{25}$ Lemonnier et al. have demonstrated that fascial fibroblasts have longer cell-doubling times, larger cell volume, and higher glucose requirements than dermis fibroblast. ${ }^{26}$

Following acute surgical injury of the abdominal fascia, neutrophils, monocytes, macrophages, and fibroblasts are involved in the healing process. ${ }^{27,28}$ However, the previously described cellular events in normal fascial healing are only comparable to a limited extent due to the use of different animal models. ${ }^{29}$ Furthermore, most of these studies investigate the fascial healing process after one week, disregarding the importance of the cellular and molecular events in the early healing stages. There is a paucity of the temporal changes of cells and related gene expressions, especially in the early postoperative period. Additionally, lipid mediators can affect cellular biology related to wound healing. ${ }^{30}$ For example, diacylglycerol, lysophosphatidic acid, arachidonic acid, and eicosanoids have been described to be involved in healing process. ${ }^{31-34}$ Few studies have illustrated the temporal healing process of fascia in abdominal wall on the molecular level.

\section{Postoperative adhesions}

Intra-abdominal adhesions can be considered as internal overhealing, ${ }^{5}$ referring to bands of fibrous tissues spanning two or more abdominal organs, although an official definition is lacking. ${ }^{35,36}$ Virtually all patients who underwent laparotomy can develop intra-abdominal adhesions, ${ }^{37}$ leading to complications such as pain, intestinal obstruction, female infertility, and difficulty in reoperation. ${ }^{38}$ Usually, postoperative intra-abdominal adhesion formation initiates from an inflammatory response caused by surgical trauma. ${ }^{39}$ A fibrin network ultimately results in permanent adhesion formation. ${ }^{40,41}$

Peritoneal adhesions can form between omentum, internal organs, and the abdominal wall. $^{42}$ To prevent peritoneal adhesions, one of the major strategies is to use physical barriers separating the surfaces of two tissues (Figure 1.2, a). Two FDA-approved barriers, Seprafilm (Genzyme Corporation) and Interceed (Johnson \& Johnson) are most commonly applied in clinical practice. However, limitations and controversies exist for both products concerning the efficacy and/or the biocompatibility. ${ }^{43}$ Therefore, a search for the ideal solid and liquid barriers remains highly relevant.

Clinically, IH can be repaired using intraperitoneal onlay mesh technique (IPOM) to achieve a tension-free repair. Intraperitoneal placement of meshes reduces the recurrence of abdominal wall hernia significantly, compared to hernia repair only with 
sutures. ${ }^{44}$ However, adhesions can form from internal organs to the IPOM mesh (Figure $1.2, \mathrm{~b}){ }^{45}$ Antiadhesive coating layers on the visceral side of IPOM meshes have been used to reduce the to-mesh adhesions (Figure 1.2, c). A large number of animal experiments have been conducted to compare the antiadhesion effect of mesh coatings. However, it is a challenge to compare mesh coatings in different animal experiments due to the lack of a thorough overview checking efficacy of an identical antiadhesive coating in various experimental conditions.

\section{In vivo animal experiments}

Animal models are commonly used for normal fascial healing, due to ethical impossibility to isolate functionally healed fascia from patients. Histological results in humans and rats seem comparable in a hernia model, facilitating the translation of animal results to humans. ${ }^{46} \mathrm{~A}$ midline incision for a colectomy has a significantly higher incidence of $\mathrm{IH}$ in humans, compared to a transverse muscle cutting incision. ${ }^{47-50}$ A colectomy animal model with midline incision of fascia could mimic both laparoscopic and open colectomy in patients using a midline fascial incision.

Several animal models have been developed for adhesion formation, using techniques including ischaemic buttons established by suturing ligation of parietal peritoneum, abrasion of peritoneum/cecum/uterine horns, suturing connection of internal organs and peritoneum, excision of peritoneum, and electrocoagulation of peritoneum. A previous study has demonstrated that the ischaemic button model is the most consistent and reproducible model compared with the abrasion of peritoneum or cecum as well as the excision of peritoneum. ${ }^{51}$ The ischaemic button model uses a suture tied around a $5 \mathrm{~mm}$ portion of the parietal peritoneum, resulting in ischaemic buttons of the ligated tissue and consequently the development of adhesions between internal organs and the ischaemic button. ${ }^{52}$ This model helps the evaluation and development of antiadhesive substances to prevent intraperitoneal adhesion formation.

Gender-related differences in animal models are an important aspect to take into account when refining animal testing. ${ }^{53}$ The ischaemic button model is frequently used for evaluating efficacy of antiadhesive substances. However, the baseline performance, in terms of quality and quantity of adhesion formation as well as IH formation, between male and female animals has never been investigated in this model before. Both male and female rats have been used in previous studies using the ischaemic button model, however, most studies only use male rats without a clear reason for the gender selection. The evaluation of the baseline performance of both male and female rats could provide evidence-based data for sex consideration for further animal experiments. 
a

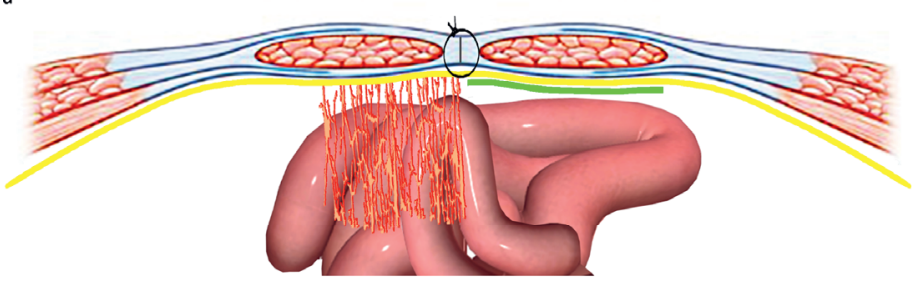

b

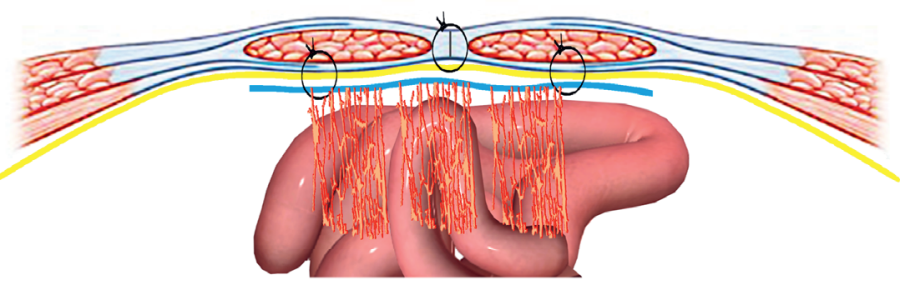

c

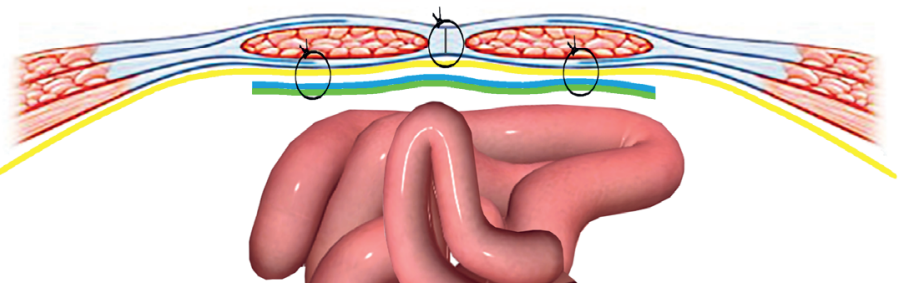

Figure 1.2 In (a), adhesions (red-yellowish strands) between small intestines and peritoneum is depicted. A barrier (green) is placed between small intestines and peritoneum preventing adhesion formation. In (b), adhesions (red-yellowish) forms between small intestines and an IPOM mesh (blue) that is used to correct an IH. In (c), antiadhesion coatings (green) on the IPOM mesh is used to prevent to-mesh-adhesion formation.

Note. This illustration is a modification from a picture of Bingöl et al. (2016). ${ }^{17}$

Animal models are essential for current biomedical research. ${ }^{54}$ Alternative methods have been developed to minimize the use of animals, such as computer models, cell and tissue cultures, alternative organisms, and human volunteers. ${ }^{55,56}$ In spite of these efforts, more than ten million animals were used for scientific research across the European Union per year. ${ }^{57}$ In order to maximize information published and avoid 
unnecessary animal studies, the ARRIVE (Animal Research: Reporting of In Vivo Experiments) guidelines have been published from 2010 and updated in 2019. With respect to animal experiments using meshes for abdominal wall hernia repair, there were hundreds of animal experiments published previously. ${ }^{29}$ Before implantation of new meshes in patients, evaluation of the efficacy and biocompatibility of these meshes in preclinical experiments is essential despite promising in vitro or ex vivo results. ${ }^{58}$ Despite more than 70 commercially available meshes, the search for an ideal surgical mesh for abdominal wall repair is ongoing and further animal experiments testing meshes will be expected. ${ }^{59}$ However, evaluation of the reporting quality of these experiments is lacking over the years.

\section{Thesis outline}

This thesis on the abdominal fascia healing process includes three sections, (I) efforts to optimize animal research, (II) the investigation of the temporal cellular and molecular changes in the normal healing process, and (III) the evaluation of preventive measures of adhesion formation within fascia healing.

Most of the chapters in this thesis include animal experiments. The reporting quality of manuscripts complying the 3Rs (Replacement, Reduction and Refinement) principles is of importance for study reproducibility. Therefore, to assess the effect of the ARRIVE guidelines on improving reporting quality of articles, Chapter 2 presents the comparison of reporting quality of publications before and after the ARRIVE guidelines were introduced, focusing on abdominal hernia repaired with meshes using animal models.

Gender differences in baseline performance, regarding the quality and quantity of intraperitoneal adhesion formation as well as the fascial healing, is an essential consideration for further animal experiments investigating antiadhesive barriers. The objective of Chapter 3 is to compare the baseline performance between male and female rats in an ischaemic button model.

IH is a common complication after abdominal operations, caused by abnormal fascial healing. Knowledge of normal fascial healing could provide prediction or treatment strategies for abnormal fascial healing. Cells and related genes play a crucial role in the fascial healing process. In Chapter 4, we present the temporal changes of cellular populations and related gene expression levels in the first postoperative week of fascia healing in rats. Furthermore, since the importance of lipids in this healing process has 
been reported in previous studies, Chapter 5 aims to provide new insight into fascial healing on the lipid level in the first postoperative week in rats.

Animal testing is of importance as a bridge connecting promising in vitro results and safe human interpretation. Peritoneal adhesion formation is one of the common complications after abdominal surgical procedures. Chapter 6 aims to compare five solid antiadhesive polymers regarding the quality and quantity of peritoneal adhesions using an ischaemic button adhesion model in rats. Using the identical ischaemic button adhesion model in rats, chapter 7 focuses on the evaluation of the antiadhesive effect of a novel hyaluronic acid gel compared to already commercially available gels.

Preventive meshes are applied clinically for enhancing weakened abdominal wall due to surgical incision. Mesh implantation can be placed prophylactically in high-risk patients developing $\mathrm{IH}$, as well as for the repair of large size IH. The placement of IPOM meshes can induce intestine-to-mesh adhesions with potential adhesion-related complications as a result. Mesh coatings could provide a physical barrier between the visceral side of the mesh and intestine, preventing adhesion-to-mesh formation. Chapter 8 aims to provide systematic evidence of the antiadhesion performance of coatings on meshes.

In Chapter 9, the main findings from the studies described in this thesis are integrated and discussed in a broader perspective. In addition, suggestions for future perspectives of tissue healing are provided. 


\section{References}

1. Ellis H. The first successful elective laparotomy. J Perioper Pract. 2008;18(5):211.

2. Centraal Bureau voor de Statistiek. Operaties in het ziekenhuis; soort opname, leeftijd en geslacht,1995-2010. CBS 2014.

3. Bonjer HJ, Bruining HA. Surgery in the Netherlands. Arch Surg. 1999;134(1):92-8.

4. Kossler-Ebs JB, Grummich K, Jensen K, Huttner FJ, Muller-Stich B, Seiler CM, et al. Incisional Hernia Rates After Laparoscopic or Open Abdominal Surgery-A Systematic Review and MetaAnalysis. World J Surg. 2016;40(10):2319-30.

5. Ellis H. Internal overhealing: the problem of intraperitoneal adhesions. World J Surg. 1980;4(3):303-6.

6. Duron JJ. Postoperative intraperitoneal adhesion pathophysiology. Colorectal Dis. 2007;9 Suppl 2: 14-24.

7. Krafts KP. Tissue repair: The hidden drama. Organogenesis. 2010;6(4):225-33.

8. Dubay DA, Wang X, Kuhn MA, Robson MC, Franz MG. The prevention of incisional hernia formation using a delayed-release polymer of basic fibroblast growth factor. Ann Surg. 2004;240(1):179-86.

9. Bordoni B, Mahabadi N, Varacallo M. Anatomy, Fascia. StatPearls. Treasure Island (FL)2020.

10. Sanders DL, Kingsnorth AN. The modern management of incisional hernias. BMJ. 2012;344:e2843.

11. Bosanquet DC, Ansell J, Abdelrahman T, Cornish J, Harries R, Stimpson A, et al. Systematic Review and Meta-Regression of Factors Affecting Midline Incisional Hernia Rates: Analysis of 14,618 Patients. PLoS One. 2015;10(9):e0138745.

12. Fink C, Baumann $P$, Wente MN, Knebel P, Bruckner T, Ulrich A, et al. Incisional hernia rate 3 years after midline laparotomy. Br J Surg. 2014;101(2):51-4.

13. Gillion JF, Sanders D, Miserez M, Muysoms F. The economic burden of incisional ventral hernia repair: a multicentric cost analysis. Hernia. 2016;20(6):819-30.

14. Poulose BK, Shelton J, Phillips S, Moore D, Nealon W, Penson D, et al. Epidemiology and cost of ventral hernia repair: making the case for hernia research. Hernia. 2012;16(2):179-83.

15. Pollock AV, Evans M. Early prediction of late incisional hernias. Br J Surg. 1989;76(9):953-4.

16. Thankam FG, Palanikumar G, Fitzgibbons RJ, Agrawal DK. Molecular Mechanisms and Potential Therapeutic Targets in Incisional Hernia. J Surg Res. 2019;236:134-43.

17. Bingöl D, Ozkaya O, Oymak MO. Surgical Anatomy of the Abdominal Wall. Springer, Cham.; 2017.

18. Caglia P, Tracia A, Borzi L, Amodeo L, Tracia L, Veroux M, et al. Incisional hernia in the elderly: risk factors and clinical considerations. Int J Surg. 2014;12 Suppl 2:S164-S9.

19. Basta MN, Kozak GM, Broach RB, Messa CAt, Rhemtulla I, DeMatteo RP, et al. Can We Predict Incisional Hernia?: Development of a Surgery-specific Decision-Support Interface. Ann Surg. 2019; 270(3):544-53.

20. Mommers EHH, van Kooten L, Nienhuijs SW, de Vries Reilingh TS, Lubbers T, Mees BME, et al. Can Electric Nose Breath Analysis Identify Abdominal Wall Hernia Recurrence and Aortic Aneurysms? A Proof-of-Concept Study. Surg Innov. 2020:1553350620917898.

21. Velnar T, Bailey T, Smrkolj V. The wound healing process: an overview of the cellular and molecular mechanisms. J Int Med Res. 2009;37(5):1528-42.

22. Muysoms FE, Antoniou SA, Bury K, Campanelli G, Conze J, Cuccurullo D, et al. European Hernia Society guidelines on the closure of abdominal wall incisions. Hernia. 2015;19(1):1-24.

23. Franz MG, Smith PD, Wachtel TL, Wright TE, Kuhn MA, Ko F, et al. Fascial incisions heal faster than skin: a new model of abdominal wall repair. Surgery. 2001;129(2):203-8.

24. Dubay DA, Wang X, Kirk S, Adamson B, Robson MC, Franz MG. Fascial fibroblast kinetic activity is increased during abdominal wall repair compared to dermal fibroblasts. Wound Repair Regen. 2004; 12(5):539-45.

25. Douglas DM. The healing of aponeurotic incisions. Br J Surg. 1952;40(159):79-84.

26. Lemonnier F, Gautier M, Wolfrom C, Lemonnier A. Some metabolic differences between human skin and aponeurosis fibroblasts in culture. J Cell Physiol. 1980;104(3):415-23. 
27. Jansen PL, Mertens Pr P, Klinge U, Schumpelick V. The biology of hernia formation. Surgery. 2004;136(1):1-4.

28. Franz MG. The biology of hernia formation. Surg Clin North Am. 2008;88(1):1-15, vii.

29. Vogels RRM, Kaufmann R, van den Hil LCL, van Steensel S, Schreinemacher MHF, Lange JF, et al. Critical overview of all available animal models for abdominal wall hernia research. Hernia. 2017;21(5):667-75.

30. Wijesinghe DS, Warncke UO, Diegelmann RF. Human as the Ultimate Wound Healing Model: Strategies for Studies Investigating the Dermal Lipidome. Curr Dermatol Rep. 2016;5(4):244-51.

31. Vaughan EM, You JS, Elsie Yu HY, Lasek A, Vitale N, Hornberger TA, et al. Lipid domain-dependent regulation of single-cell wound repair. Mol Biol Cell. 2014;25(12):1867-76.

32. Demoyer JS, Skalak TC, Durieux ME. Lysophosphatidic acid enhances healing of acute cutaneous wounds in the mouse. Wound Repair Regen. 2000;8(6):530-7.

33. Oh SY, Lee SJ, Jung YH, Lee HJ, Han HJ. Arachidonic acid promotes skin wound healing through induction of human MSC migration by MT3-MMP-mediated fibronectin degradation. Cell Death Dis. 2015;6:e1750.

34. Dhall S, Wijesinghe DS, Karim ZA, Castro A, Vemana HP, Khasawneh FT, et al. Arachidonic acidderived signaling lipids and functions in impaired healing. Wound Repair Regen. 2015;23(5):644-56.

35. Bruggmann D, Tchartchian G, Wallwiener M, Munstedt K, Tinneberg HR, Hackethal A. Intraabdominal adhesions: definition, origin, significance in surgical practice, and treatment options. Dtsch Arztebl Int. 2010;107(44):769-75.

36. Tabibian N, Swehli E, Boyd A, Umbreen A, Tabibian JH. Abdominal adhesions: A practical review of an often overlooked entity. Ann Med Surg (Lond). 2017;15:9-13.

37. Stommel MWJ, Ten Broek RPG, Strik C, Slooter GD, Verhoef C, Grunhagen DJ, et al. Multicenter Observational Study of Adhesion Formation After Open-and Laparoscopic Surgery for Colorectal Cancer. Ann Surg. 2018;267(4):743-8.

38. ten Broek RP, Issa Y, van Santbrink EJ, Bouvy ND, Kruitwagen RF, Jeekel J, et al. Burden of adhesions in abdominal and pelvic surgery: systematic review and met-analysis. BMJ. 2013;347:f5588.

39. Maciver AH, McCall M, James Shapiro AM. Intra-abdominal adhesions: cellular mechanisms and strategies for prevention. Int J Surg. 2011;9(8):589-94.

40. Holmdahl L. The role of fibrinolysis in adhesion formation. Eur J Surg Suppl. 1997(577):24-31. 405

41. Boland GM, Weigel RJ. Formation and prevention of postoperative abdominal adhesions. J Surg Res. 2006;132(1):3-12.

42. Arung W, Meurisse M, Detry O. Pathophysiology and prevention of postoperative peritoneal adhesions. World J Gastroenterol. 2011;17(41):4545-53.

43. Ward BC, Panitch A. Abdominal adhesions: current and novel therapies. J Surg Res. 2011;165(1): 91-111.

44. Jairam AP, Timmermans L, Eker HH, Pierik R, van Klaveren D, Steyerberg EW, et al. Prevention of incisional hernia with prophylactic onlay and sublay mesh reinforcement versus primary suture only in midline laparotomies (PRIMA): 2-year follow-up of a multicentre, double-blind, randomised controlled trial. Lancet. 2017;390(10094):567-76.

45. Moreno-Egea A, Bustos JA, Girela E, Aguayo-Albasini JL. Long-term results of laparoscopic repair of incisional hernias using an intraperitoneal composite mesh. Surg Endosc. 2010;24(2):359-65.

46. van den Hil LCL, Vogels RRM, van Barneveld KWY, Gijbels MJJ, Peutz-Kootstra CJ, Cleutjens JPM, et al. Comparability of histological outcomes in rats and humans in a hernia model. J Surg Res. 2018;229:271-6.

47. Navaratnam AV, Ariyaratnam R, Smart NJ, Parker M, Motson RW, Arulampalam TH. Incisional hernia rate after laparoscopic colorectal resection is reduced with standardisation of specimen extraction. Ann R Coll Surg Engl. 2015;97(1):17-21.

48. Lee L, Mata J, Droeser RA, Kaneva P, Liberman S, Charlebois P, et al. Incisional Hernia After Midline Versus Transverse Specimen Extraction Incision: A Randomized Trial in Patients Undergoing Laparoscopic Colectomy. Ann Surg. 2018;268(1):41-7. 
49. Braga M, Frasson M, Vignali A, Zuliani W, Civelli V, Di Carlo V. Laparoscopic vs. open colectomy in cancer patients: long-term complications, quality of life, and survival. Dis Colon Rectum. 2005;48(12):2217-23.

50. Ihedioha U, Mackay G, Leung E, Molloy RG, O'Dwyer PJ. Laparoscopic colorectal resection does not reduce incisional hernia rates when compared with open colorectal resection. Surg Endosc. 2008;22(3):689-92.

51. Whang SH, Astudillo JA, Sporn E, Bachman SL, Miedema BW, Davis W, et al. In search of the best peritoneal adhesion model: comparison of different techniques in a rat model. $\mathrm{J}$ Surg Res. 2011;167(2):245-50.

52. Rajab TK, Wauschkuhn CA, Smaxwil L, Kraemer B, Wallwiener M, Wallwiener CW. An improved model for the induction of experimental adhesions. J Invest Surg. 2010;23(1):35-9.

53. Miller LR, Marks C, Becker JB, Hurn PD, Chen WJ, Woodruff T, et al. Considering sex as a biological variable in preclinical research. FASEB J. 2017;31(1):29-34.

54. Franco NH. Animal Experiments in Biomedical Research: A Historical Perspective. Animals (Basel). 2013;3(1):238-73.

55. Doke SK, Dhawale SC. Alternatives to animal testing: A review. Saudi Pharm J. 2015;23(3):223-9.

56. Combes RD, Berridge T, Connelly J, Eve MD, Garner RC, Toon S, et al. Early microdose drug studies in human volunteers can minimise animal testing: Proceedings of a workshop organised by Volunteers in Research and Testing. Eur J Pharm Sci. 2003;19(1):1-11.

57. Taylor K, Alvarez LR. An Estimate of the Number of Animals Used for Scientific Purposes Worldwide in 2015. Altern Lab Anim. 2019;47(5-6):196-213.

58. Bringman S, Conze J, Cuccurullo D, Deprest J, Junge K, Klosterhalfen B, et al. Hernia repair: the search for ideal meshes. Hernia. 2010;14(1):81-7.

59. Baylon K, Rodriguez-Camarillo P, Elias-Zuniga A, Diaz-Elizondo JA, Gilkerson R, Lozano K. Past, Present and Future of Surgical Meshes: A Review. Membranes (Basel). 2017;7(3). 
Part I

The ideal animal model 



\section{Chapter 2}

Do ARRIVE guidelines improve the quality of mesh repair experiments in animals?

Inadequate awareness of adherence to ARRIVE guidelines, regarding reporting quality of hernia models repaired with meshes: a systematic review

Hong Liu, Maurits-Jan J. C.A.M. Gielen, Joanna W.A.M. Bosmans, Bjorn Winkens, Nicole D. Bouvy 


\section{Abstract}

\section{Purpose}

Research papers involving animal studies often display poor reporting standards, leading to lower study reproducibility. We aim to determine the difference in reporting animal studies regarding abdominal wall hernia repair with mesh placement, before and after the publication of ARRIVE-2010 (Animal Research: Reporting of In Vivo Experiments) guidelines. Furthermore, we aim to present the most up-to-date reporting quality using the updated ARRIVE-2020 as criteria.

\section{Methods}

All animal studies concerning hernia repair with meshes were systematically searched. Articles published in the five years leading up to the ARRIVE-2010 (pre-ARRIVE) and articles within the last five years until the updated ARRIVE 2.0 (post-ARRIVE) were compared for overall species and specific species separately. Articles published last year were evaluated for presenting fully reported (sub)items.

\section{Results}

The number of fully reported (sub)items per article was on average significantly higher for pre-ARRIVE than post-ARRIVE for overall species (mean (SD)=14.0 (2.8) vs. 12.6 (2.5), $P<0.001$ ). The same applies to rabbit (mean $(\mathrm{SD})=14.8$ (2.6) vs. 12.6 (2.6), $P=0.001)$ and pig studies (mean $(\mathrm{SD})=14.5(2.7)$ vs. $11.6(2.6), P=0.004)$, with no significance in rat studies (mean (SD) $=13.6(2.9)$ vs. $12.9(2.3), P=0.076)$. Significance was found in several (sub)items between pre-ARRIVE and post-ARRIVE $(n=7,3,8$, and 3 for overall species, rat, rabbit, and pig studies, respectively).

\section{Conclusion}

General reporting quality of animal experiments has been improved markedly by ARRIVE guidelines. However, more improvements are required considering the arrival of ARRIVE 2.0 guidelines. 


\section{Introduction}

Over the last centuries, animal testing has solidified its position in the medical world as an accessible replacement for humans in experimental research. ${ }^{1}$ Despite the development of non-animal models as alternatives replacing animal experiments, such as computer models, tissue and cell cultures, and human volunteer studies, animal experiments remain indispensable. ${ }^{2,3}$ However, inadequate reporting of key aspects in animal studies acts as one of the most common barriers against study reproducibility. ${ }^{4,5}$ Additionally, animal research often yields inconclusive results under the pretence that there is a need for more future research. These publications can lead to significant growth in financial costs, ethical costs, and the number of animals being used. ${ }^{6}$ Furthermore, errors or omissions in reporting, pertaining to study design, data selection, or interpretation of results and effect sizes, introduce unwanted heterogeneity among animal studies. ${ }^{7}$

Earlier research evaluating the use of animal models has shown that translatability of preclinical research to humans is largely dependent on the reporting of the same fundamental aspects normally reported in clinical research. ${ }^{8,9}$ Following these results, increasing the standards of reporting in animal research should lead to more fruitful research in the future. Therefore, to improve reporting quality in all studies using animals, the ARRIVE (Animal Research: Reporting of In Vivo Experiments) guidelines for reporting were initially published in 2010 (ARRIVE-2010), updated in July 2019 and formally published in July 2020 (ARRIVE-2020). ${ }^{10,11}$ The ARRIVE-2010 guidelines consist of 20 items that should be included in animal research manuscripts, describing comprehensive reports from study design and study samples to animal quality of life and translatability. The ARRIVE-2020 guidelines, updated with prioritised items and new added information, consist of 10 essential items and 11 recommended items. By improving reporting, ARRIVE aims to improve the quality of life for animals, quality of animal experiments, and reduction of animals needed in research. These considerations and limitations are based on the three Rs principle (focusing on the replacement, reduction, and refinement of animals in animal studies) and are basic principles of humane experimental techniques. ${ }^{12,13}$ Animal experiments of poor quality and with inconclusive results fail to adhere to the three Rs and waste countless lives of animals, adding to the existing controversy of experimentation on animals. $^{1,10,13,14}$

There are hundreds of animal experiments published previously with regard to the use of meshes for abdominal wall hernia repair in animal models. ${ }^{15}$ The search for an ideal surgical mesh for abdominal wall repair is ongoing due to mesh-related complications including hernia recurrence, infection, and to-mesh adhesions, despite more than 70 commercially available meshes. ${ }^{16}$ Before implementing new meshes in clinical 
practice, evaluation of the efficacy and biocompatibility of these meshes in preclinical experiments is essential. ${ }^{17}$ However, reporting quality of these experiments is lacking over the years.

Therefore, our primary aim is to determine the difference in reporting of animal studies regarding abdominal wall hernia repair with mesh placement, between articles published before the publication of ARRIVE-2010 (pre-ARRIVE) and after the publication of ARRIVE-2010 (post-ARRIVE). Moreover, our secondary aim is to present the reporting quality of articles published most recently, using the updated ARRIVE-2020 guidelines as criteria.

\section{Methods}

\section{Inclusion and exclusion criteria}

All studies concerning hernia repair with meshes in animals were eligible for inclusion. Furthermore, studies containing in vitro analyses or human trials were included, as long as authentic animal experiments were performed and described in the article. Reviews were excluded for the lack of description per individual animal experiment and the risk of multiple publication bias. ${ }^{18}$ Case reports were excluded due to lack of controlled experimental setting and protocol. ${ }^{19}$ Additionally, studies concerning hernia repair without placement of a mesh or barrier in vivo were excluded, as well as studies examining hernia repairs not associated with the abdominal wall (i.e. inguinal hernia, hiatal hernia, or pelvic enhancement for preventing pelvic organ prolapse). Articles that were not written in or translated to English were excluded, as well as papers without available full-text articles, letters to editors, or conference abstracts.

\section{Search strategies}

Pubmed, Embase, and Web of Science were systematically searched and updated until July 21, 2020 using a structured search query (Supplementary Table S2.1). The strategies for searching all animal experiments followed the SYRCLE (SYstematic Review Center for Laboratory animal Experimentation) guidance. The handling and removal of duplicate articles were performed independently by two researchers (HL and MG), using EndNote's own 'Find duplicates'-function, as well as removing duplicates by hand. Duplicates were manually removed by sorting and comparing the list of both authors and title; and/or by journal, issue and page number. 


\section{Study selection}

The newly formed list of papers was screened for inclusion and exclusion criteria within the title and abstract by two authors (HL and MG), eventually comparing both results. The differences in the inclusion of articles were discussed, after which a consensus between the two authors was met and one list was created. Taking into account the publication date of the original ARRIVE guidelines in July 2010 and the updated ARRIVE guidelines in July 2019, only articles published between July 2005 and June 2010 and between July 2014 and June 2019 were selected for comparison. In that way, a pre-ARRIVE group (2005-2010) and post-ARRIVE group (2014-2019) consisting of five years each, was created. Additionally, articles published between July 2019 and the new searching date (July 2020) were selected for illustrating the reporting quality of articles most recently published (post-2019 group).

\section{Evaluation}

Articles in both pre-ARRIVE and post-ARRIVE groups were evaluated using a standardized scoring form (Supplementary Table S2.2, a) on adherence to the ARRIVE-2010 guidelines, consisting of 20 items that are composed of 38 (sub)items. Articles in post-2019 group were evaluated by the essential 10 items (consisting of 23 subitems) in the ARRIVE-2020 guidelines (Supplementary Table S2.2, b). Most of these essential (sub)items overlap with the items/subitems described in the previous ARRIVE-2010 guidelines.

(Sub)items suited to be answered with 'yes' or 'no' were scored with 2 (fully reported) or 0 (not reported). An intermediate score of 1 was used in (sub)items that could be evaluated as 'partially reported', where a score 2 referred to 'fully reported' and a score 0 referred to 'not reported at all'. Additionally, a score point-scale of 0 to 3 was used in (sub)items consisting of a wider range of characteristics, receiving points based on the number of different characteristics reported. By using a broader scale, differences in reporting quality, and therefore scores, can be noticed more readily. Scores were assigned based on whether the information was reported, not on the actual influence this information could have had on the experiment. As an example, an article mentioning that no blinding took place, still received points for having reported it, even though blinding the experiment would have increased quality of the study design. The authors who had to score the articles used ARRIVE's list of examples per (sub)item in order to concretize specific guidelines, to ensure consistency, and to help determine whether a (sub)item should be scored as 'reported', 'partially reported', or 'not reported'. 10,11,20

After separate evaluation of the included articles by two researchers (HL and MG), results were compared. Inter-observer discrepancy of each (sub)item on each included 
article was solved by a discussion between the two researchers (HL and MG) and a final scoring of the articles was established.

\section{Data synthesis and statistical analysis}

All papers were assessed according to our scoring form. The difference in mean number of fully reported items/subitems per article between pre-ARRIVE and postARRIVE group was assessed using independent-samples t-test, where normality of the data within each group was confirmed with both Shapiro-Wilk test and Q-Q Plots. Differences in categorical variables between pre- and post-ARRIVE articles were assessed using Chi-square test (with Yates' correction for binary outcomes), or Fisher's exact test (Fisher-Freeman-Halton in case outcome has three or more categories), when applicable.

Above comparisons were performed using all animal species without distinction. In addition, the same comparisons were performed for specific species separately (e.g. including only studies with rats in the comparison between pre-ARRIVE and postARRIVE articles).

Two-sided p-values $\leq 0.05$ were considered statistically significant. No correction for multiple testing was performed due to the explorative nature of this study. Statistical analysis was performed using IBM SPSS Statistics for Windows (version 25.0, Armonk, NY, USA, IBM Corp.).

\section{Results}

\section{Outcome of numbers of included articles}

Initially, a total of 3754 articles were found using the aforementioned search query. After removal of duplicates, 1991 articles were left for the selection and title/abstract screening. Additional 9 articles were found meeting inclusion criteria from other sources, for example, reference and articles included in other reviews. Finally, a total of 267 papers, 107 pre-ARRIVE articles and 160 post-ARRIVE articles, were left to be analyzed by means of our scoring form (Figure 2.1). The most recent 16 articles were left in the post-2019 group for evaluation using the essential 10 items in ARRIVE2020 . 


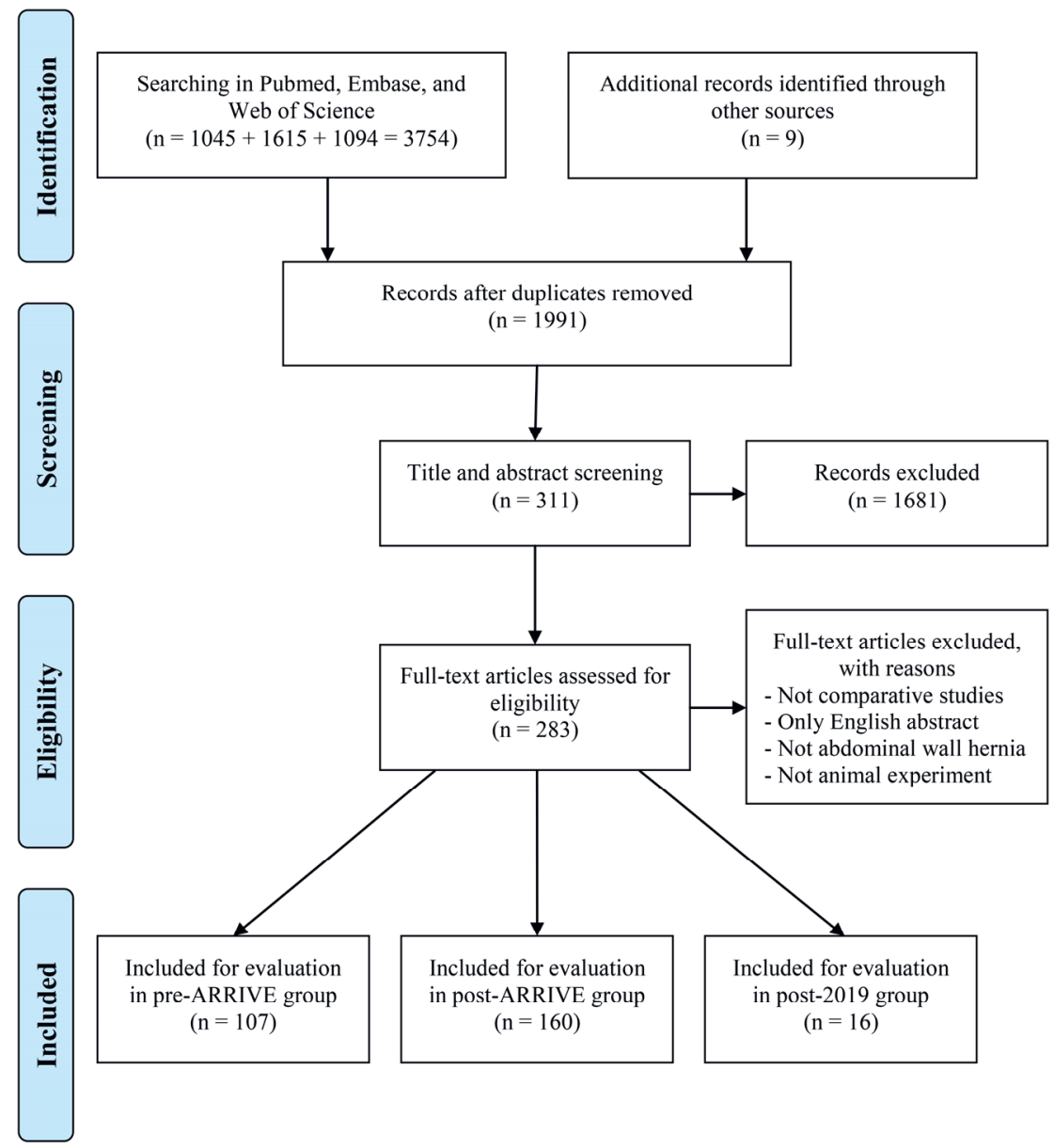

Figure 2.1 Workflow for the inclusion of articles.

\section{Distribution of animals in pre- and post-ARRIVE}

For the 267 articles that were scored, distribution of animal species that were used in pre-ARRIVE and post-ARRIVE articles are displayed (Figure 2.2.) The three most common animal species used were rats, rabbits, and pigs. After the publication of ARRIVE-2010, the proportion of studies that used rats has slightly increased from $55 / 107(51 \%)$ to $88 / 160(55 \%)$, while the proportion slightly decreased from $29 / 107$ $(27 \%)$ to $37 / 160(23 \%)$ for rabbits and considerable decreased from $17 / 107$ (16\%) to $18 / 160(11 \%)$ for pigs. 

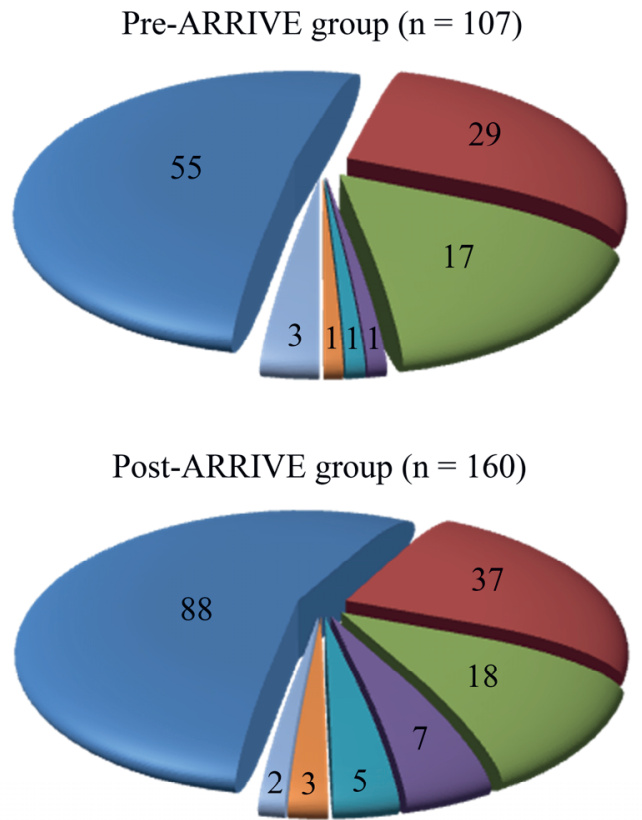

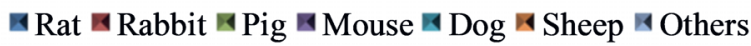

Figure 2.2 Distribution of animal species in pre-ARRIVE and post-ARRIVE groups.

\section{Number of fully reported (sub)items per article}

Ideally, a total of 38 (sub)items should be fully reported according to ARRIVE-2010. A total of 35 (sub)items were analyzed in this study for all articles in pre- and postARRIVE. Three subitems (10c, 15b, and 17b) were evaluated but not analyzed as they do not apply to all included articles in both pre- and post-ARRIVE (Subitem10c indicating the number of independent replications of each experiment; Subitem 15b referring to explain why animals or data were not included in the analysis; Subitem 17b describing any modifications to the experimental protocols made to reduce adverse events).

Compared to the pre-ARRIVE group for overall studies, the mean number of fully reported (sub)items per article in the post-ARRIVE group increased significantly $(P<0.001)$. In the comparison for studies of specific species, the mean numbers had increased significantly for the rabbit and the pig studies whereas it had no significant difference for rat studies (rat, $P=0.076$; rabbit, $P=0.001$; pig, $P=0.004$ ) (Table 2.1). 
Table 2.1 Mean numbers of fully reported (sub)items per article between pre-ARRIVE and post-ARRIVE.

\begin{tabular}{lccc}
\hline & $\begin{array}{c}\text { Pre-ARRIVE } \\
\text { Mean } \pm \mathrm{SD}\end{array}$ & $\begin{array}{c}\text { Post-ARRIVE } \\
\text { Mean } \pm \mathrm{SD}\end{array}$ & $P$-value \\
\hline Overall species & $12.6 \pm 2.5$ & $14.0 \pm 2.8$ & $<0.001$ \\
Rat studies & $12.9 \pm 2.3$ & $13.6 \pm 2.9$ & 0.076 \\
Rabbit studies & $12.6 \pm 2.6$ & $14.8 \pm 2.6$ & 0.001 \\
Pig studies & $11.6 \pm 2.6$ & $14.5 \pm 2.7$ & 0.004 \\
\hline
\end{tabular}

\section{ARRIVE guidelines clearly mentioned in articles}

A total of six out of 160 articles for post-ARRIVE group, belonging to five research teams, clearly mentioned that the reporting of their study was adhered to ARRIVE guidelines. Only one out of 16 papers for the ARRIVE-2020 group clearly reported the adherence to ARRIVE guidelines.

\section{Per-item-analysis}

Overall changes for all (sub)items based on ARRIVE-2010 in all animal species, rats, rabbits, and pigs are displayed in Table 2.2 and Supplementary Tables S2.3-S2.5, respectively. The number of (sub)items showing significant difference between preARRIVE and post-ARRIVE was seven for overall species, three for rat studies, eight for rabbit studies, and three for pig studies. Most (sub)items showed significant increase of fully reported scores for post-ARRIVE group rather than decrease, demonstrated in the description column in Table 2.2 and Supplementary Tables S2.3S2.5.

Table 2.2 Comparison of articles per (sub)item between pre-ARRIVE and post-ARRIVE for overall studies. Data are displayed as percentages of articles per score (or 'reported') of all the articles in that score. *represents essential (sub)items in the ARRIVE-2020 guidelines.

\begin{tabular}{|c|c|c|c|c|c|c|c|}
\hline \multirow{2}{*}{$\begin{array}{l}\text { Item } \\
1\end{array}$} & \multirow{2}{*}{$\begin{array}{c}\text { Score } \\
0,\end{array}$} & \multicolumn{2}{|c|}{$\begin{array}{c}\text { Pre-ARRIVE } \\
\mathrm{n}=107 \text { per item }\end{array}$} & \multicolumn{2}{|c|}{$\begin{array}{c}\text { Post-ARRIVE } \\
\mathrm{n}=160 \text { per item }\end{array}$} & \multirow{2}{*}{$\begin{array}{c}P \text {-value } \\
0.012\end{array}$} & \multirow{3}{*}{$\begin{array}{l}\text { Description } \\
\text { Score } 1 \text { presents the most significant } \\
\text { difference }\end{array}$} \\
\hline & & 58 & $54 \%$ & 78 & $49 \%$ & & \\
\hline & 1 & 0 & $0 \%$ & 11 & $7 \%$ & & \\
\hline & 2 , & 49 & $46 \%$ & 71 & $44 \%$ & & \\
\hline \multirow[t]{3}{*}{2} & 0 , & 0 & $0 \%$ & 0 & $0 \%$ & 0.798 & \\
\hline & 1 , & 64 & $60 \%$ & 99 & $62 \%$ & & \\
\hline & 2 & 43 & $40 \%$ & 61 & $38 \%$ & & \\
\hline \multirow[t]{2}{*}{$3 a$} & No, & 0 & $0 \%$ & 0 & $0 \%$ & & All articles with 'fully reported' for pre- \\
\hline & Yes, & 107 & $100 \%$ & 160 & $100 \%$ & & ARRIVE \\
\hline \multirow[t]{3}{*}{$3 b$} & 0 & 67 & $63 \%$ & 104 & $65 \%$ & 0.862 & \\
\hline & 1 , & 27 & $25 \%$ & 40 & $25 \%$ & & \\
\hline & 2 & 13 & $12 \%$ & 16 & $10 \%$ & & \\
\hline \multirow[t]{2}{*}{4} & No, & 0 & $0 \%$ & 1 & $1 \%$ & 1.000 & All articles with 'fully reported' for pre- \\
\hline & Yes, & 107 & $100 \%$ & 159 & $99 \%$ & & ARRIVE \\
\hline
\end{tabular}


Table 2.2 (continued)

\begin{tabular}{|c|c|c|c|c|c|c|c|}
\hline \multirow{2}{*}{$\begin{array}{l}\text { Item } \\
5\end{array}$} & \multirow{2}{*}{$\begin{array}{c}\text { Score } \\
0,\end{array}$} & \multicolumn{2}{|c|}{$\begin{array}{c}\text { Pre-ARRIVE } \\
\mathrm{n}=107 \text { per item }\end{array}$} & \multicolumn{2}{|c|}{$\begin{array}{l}\text { Post-ARRIVE } \\
\mathrm{n}=160 \text { per item }\end{array}$} & \multirow{3}{*}{$\begin{array}{c}P \text {-value } \\
0.016\end{array}$} & \multirow{4}{*}{$\begin{array}{l}\text { Description } \\
\text { Score } 1 \text { presents the most significant } \\
\text { difference }\end{array}$} \\
\hline & & 2 & $2 \%$ & 7 & $4 \%$ & & \\
\hline & 1 & 56 & $52 \%$ & 56 & $35 \%$ & & \\
\hline & 2 & 49 & $46 \%$ & 97 & $61 \%$ & & \\
\hline \multirow[t]{2}{*}{$6 a^{*}$} & No, & 7 & $7 \%$ & 2 & $1 \%$ & 0.450 & $>90 \%$ articles with 'fully reported' for pre- \\
\hline & Yes, & 100 & $93 \%$ & 158 & $99 \%$ & & ARRIVE \\
\hline \multirow[t]{3}{*}{$6 \mathrm{~b}$} & 0 & 44 & $41 \%$ & 66 & $41 \%$ & 1.000 & \\
\hline & 1 , & 58 & $54 \%$ & 87 & $54 \%$ & & \\
\hline & 2 & 5 & $5 \%$ & 7 & $4 \%$ & & \\
\hline \multirow[t]{2}{*}{$6 c^{*}$} & No, & 99 & $93 \%$ & 145 & $91 \%$ & 0.588 & \\
\hline & Yes, & 8 & $7 \%$ & 15 & $9 \%$ & & \\
\hline \multirow[t]{4}{*}{$7 a^{*}$} & 0 & 1 & $1 \%$ & 1 & $1 \%$ & 0.001 & Score 2 and 3 present the most significant \\
\hline & 1 , & 12 & $11 \%$ & 25 & $16 \%$ & & difference \\
\hline & 2 & 73 & $68 \%$ & 72 & $45 \%$ & & \\
\hline & 3 & 21 & $20 \%$ & 62 & $39 \%$ & & \\
\hline \multirow[t]{2}{*}{$7 b^{*}$} & No, & 107 & $100 \%$ & 160 & $100 \%$ & & None reported \\
\hline & Yes, & 0 & $0 \%$ & 0 & $0 \%$ & & \\
\hline \multirow[t]{3}{*}{$7 c^{*}$} & 0 & 74 & $69 \%$ & 121 & $76 \%$ & 0.329 & \\
\hline & 1, & 29 & $27 \%$ & 31 & $19 \%$ & & \\
\hline & 2 & 4 & $4 \%$ & 8 & $5 \%$ & & \\
\hline \multirow[t]{3}{*}{$7 d^{*}$} & 0 & 105 & $98 \%$ & 160 & $100 \%$ & 0.160 & \\
\hline & 1, & 2 & $2 \%$ & 0 & $0 \%$ & & \\
\hline & 2 & 0 & $0 \%$ & 0 & $0 \%$ & & \\
\hline \multirow[t]{4}{*}{$8 a^{*}$} & 0 & 0 & $0 \%$ & 0 & $0 \%$ & & \\
\hline & 1, & 8 & $7 \%$ & 13 & $8 \%$ & 0.105 & \\
\hline & 2 & 80 & $75 \%$ & 101 & $63 \%$ & & \\
\hline & 3 & 19 & $18 \%$ & 46 & $29 \%$ & & \\
\hline \multirow[t]{3}{*}{$8 b^{*}$} & 0 & 67 & $63 \%$ & 82 & $51 \%$ & 0.189 & \\
\hline & 1, & 36 & $34 \%$ & 69 & $43 \%$ & & \\
\hline & 2 & 4 & $4 \%$ & 9 & $6 \%$ & & \\
\hline \multirow[t]{3}{*}{$9 a$} & 0 & 76 & $71 \%$ & 110 & $69 \%$ & 0.924 & \\
\hline & 1, & 29 & $27 \%$ & 47 & $29 \%$ & & \\
\hline & 2 & 2 & $2 \%$ & 3 & $2 \%$ & & \\
\hline \multirow[t]{4}{*}{$9 b$} & 0 & 45 & $42 \%$ & 78 & $49 \%$ & & \\
\hline & 1, & 33 & $31 \%$ & 46 & $29 \%$ & 0.347 & \\
\hline & 2 & 28 & $26 \%$ & 31 & $19 \%$ & & \\
\hline & 3 & 1 & $1 \%$ & 5 & $3 \%$ & & \\
\hline \multirow[t]{3}{*}{$9 c$} & 0 & 54 & $50 \%$ & 60 & $38 \%$ & & Score 2 presents the most significant \\
\hline & 1 , & 42 & $39 \%$ & 48 & $30 \%$ & $<0.001$ & difference \\
\hline & 2 & 11 & $10 \%$ & 52 & $33 \%$ & & \\
\hline \multirow[t]{3}{*}{$10 a^{*}$} & 0 , & 2 & $2 \%$ & 5 & $3 \%$ & 0.501 & \\
\hline & 1 , & 14 & $13 \%$ & 14 & $9 \%$ & & \\
\hline & 2 & 91 & $85 \%$ & 141 & $88 \%$ & & \\
\hline \multirow[t]{2}{*}{$10 \mathrm{~b} *$} & No, & 100 & $93 \%$ & 151 & $94 \%$ & 0.757 & \\
\hline & Yes, & 7 & $7 \%$ & 9 & $6 \%$ & & \\
\hline $11 a^{*}$ & No, & 97 & $91 \%$ & 152 & $95 \%$ & 0.165 & \\
\hline & Yes, & 10 & $9 \%$ & 8 & $5 \%$ & & \\
\hline $11 b^{*}$ & No, & 89 & $83 \%$ & 150 & $94 \%$ & 0.006 & Pre-ARRIVE has more 'fully reported' \\
\hline & Yes, & 18 & $17 \%$ & 10 & $6 \%$ & & articles than post-ARRIVE \\
\hline $12 *$ & No, & 0 & $0 \%$ & 0 & $0 \%$ & & All articles with 'fully reported' for pre- \\
\hline & Yes, & 107 & $100 \%$ & 160 & $100 \%$ & & ARRIVE \\
\hline
\end{tabular}


Table 2.2 (continued)

\begin{tabular}{|c|c|c|c|c|c|c|c|}
\hline \multirow{2}{*}{$\begin{array}{l}\text { Item } \\
13 a^{*}\end{array}$} & \multirow{3}{*}{$\begin{array}{c}\text { Score } \\
\text { No, } \\
\text { Yes, }\end{array}$} & \multicolumn{2}{|c|}{$\begin{array}{l}\text { Pre-ARRIVE } \\
\mathrm{n}=107 \text { per item }\end{array}$} & \multicolumn{2}{|c|}{$\begin{array}{l}\text { Post-ARRIVE } \\
\mathrm{n}=160 \text { per item }\end{array}$} & \multirow{3}{*}{$\begin{array}{c}P \text {-value } \\
0.374\end{array}$} & \multirow{3}{*}{$\begin{array}{l}\text { Description } \\
>90 \% \text { articles with 'fully reported' for pre- } \\
\text { ARRIVE }\end{array}$} \\
\hline & & 9 & $8 \%$ & 9 & $6 \%$ & & \\
\hline & & 98 & $92 \%$ & 151 & $94 \%$ & & \\
\hline \multirow[t]{2}{*}{$13 b^{*}$} & No, & 106 & $99 \%$ & 157 & $98 \%$ & 0.916 & \\
\hline & Yes, & 1 & $1 \%$ & 3 & $2 \%$ & & \\
\hline \multirow[t]{2}{*}{$13 c^{*}$} & No, & 84 & $79 \%$ & 104 & $65 \%$ & 0.180 & \\
\hline & Yes, & 23 & $21 \%$ & 56 & $35 \%$ & & \\
\hline \multirow[t]{3}{*}{14} & 0 & 90 & $84 \%$ & 119 & $74 \%$ & 0.069 & \\
\hline & 1, & 17 & $16 \%$ & 41 & $26 \%$ & & \\
\hline & 2 & 0 & $0 \%$ & 0 & $0 \%$ & & \\
\hline \multirow[t]{2}{*}{$15 a^{*}$} & No, & 44 & $41 \%$ & 69 & $43 \%$ & 0.745 & \\
\hline & Yes, & 63 & $59 \%$ & 91 & $57 \%$ & & \\
\hline \multirow[t]{2}{*}{$16^{*}$} & No, & 32 & $30 \%$ & 33 & $21 \%$ & 0.083 & \\
\hline & Yes, & 75 & $70 \%$ & 127 & $79 \%$ & & \\
\hline \multirow[t]{2}{*}{$17 \mathrm{a}$} & No, & 23 & $21 \%$ & 41 & $26 \%$ & 0.439 & \\
\hline & Yes, & 84 & $79 \%$ & 119 & $74 \%$ & & \\
\hline \multirow[t]{2}{*}{$18 \mathrm{a}$} & No, & 0 & $0 \%$ & 0 & $0 \%$ & & All articles with 'fully reported' for pre- \\
\hline & Yes, & 107 & $100 \%$ & 160 & $100 \%$ & & ARRIVE \\
\hline \multirow[t]{2}{*}{$18 \mathrm{~b}$} & No, & 54 & $50 \%$ & 60 & $38 \%$ & 0.036 & \\
\hline & Yes, & 53 & $50 \%$ & 100 & $63 \%$ & & \\
\hline \multirow[t]{2}{*}{$18 \mathrm{c}$} & No, & 100 & $93 \%$ & 150 & $94 \%$ & 0.924 & \\
\hline & Yes, & 7 & $7 \%$ & 10 & $6 \%$ & & \\
\hline \multirow[t]{2}{*}{19} & No, & 60 & $56 \%$ & 76 & $48 \%$ & 0.170 & \\
\hline & Yes, & 47 & $44 \%$ & 84 & $53 \%$ & & \\
\hline \multirow[t]{3}{*}{20} & 0 & 58 & $54 \%$ & 17 & $11 \%$ & $<0.001$ & Score 0 presents the most significant \\
\hline & 1 , & 30 & $28 \%$ & 59 & $37 \%$ & & difference \\
\hline & 2 & 19 & $18 \%$ & 84 & $53 \%$ & & \\
\hline
\end{tabular}

The number of (sub)items fully reported in all articles in the post-2019 group

A total of 21 (sub)items were evaluated according to essential items in ARRIVE-2020. Two subitems, 6b (primary outcome measure in hypothesis-testing studies) and 10b (effect size with a confidence interval if applicable) in the ARRIVE-2020, were not evaluated since these two subitems does not always apply to our included articles in post-2019. Only eight out of 21 (sub)items in the post-2019 group has more than half rate being fully reported. The number of (sub)items fully reported in all articles in the post-2019 group is displayed in Figure 2.3. 


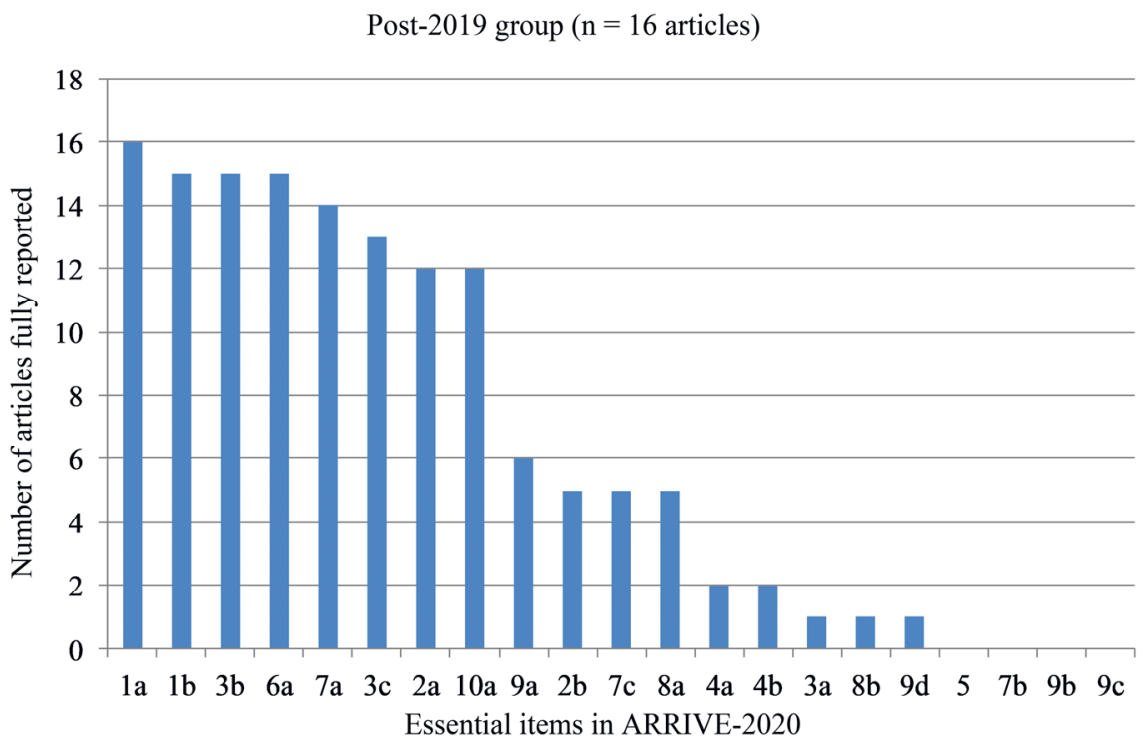

Figure 2.3 The number of articles fully reported per (sub)item in the post-2019 group.

Items 1 - 4: title, abstract, and introduction

Items 1 to 4 , referring to the reporting of title, abstract, background, and objectives respectively, displayed no significant difference between pre-ARRIVE and postARRIVE articles, except item 1 (title details). Item 1 showed a significant difference between pre- and post-ARRIVE for overall studies $(P=0.012)$, as well as for rabbitcentered $(P=0.037)$ studies and pig-centered studies $(P=0.004)$, but this difference was not found in studies focusing on rat species. The rate of articles fully reported in the post-ARRIVE group showed no significant improvement for overall species and rat studies, however, a significant decrease for rabbit studies while a significant increase for pig studies for post-ARRIVE were found for item 1. (Sub)items 3a (referring to sufficient scientific background) and 4 were reported in virtually all articles, before and after ARRIVE-2010.

Items 5 - 7: ethical statement, study design, and experimental procedures

Subitem $7 \mathrm{~b}$ (referring to time of day when conducting experiments) hasn't been reported in any of the 267 articles in pre- and post-ARRIVE groups. Item 5 stating ethical approval was found without significant difference for rat and pig studies. However, a significant difference was found in the overall species $(P=0.016)$ and rabbit sub-group $(P<0.001)$ for post-ARRIVE. A significantly increased rate of articles fully reported in this item was found for post-ARRIVE in only rabbit studies but not studies 
for overall species and other species. Subitem 7a displaying how animal experiments were performed, which consisted of a 0-to-3-point scale, was found with a significant difference between the pre-ARRIVE group and post-ARRIVE group overall $(P=0.001)$, as well as for the rat group $(P=0.004)$ and the rabbit group specifically $(P=0.023)$. Similarly, the rate of articles fully reported increased significantly for overall species, rat studies, and rabbit studies, but not pig studies.

\section{Items 8 - 9: experimental animals, housing conditions and husbandry}

Subitem 8 a regarding animal descriptors, such as the specific details of animal use and handling, is considered one of the most important causes of variability in all animal research. ${ }^{21-23}$ While this subitem could be rated a maximum of 3 points with score 2 approximating the fully reported, the score of the most articles was $\geq 2$ for both preARRIVE and post-ARRIVE, with no significant improvement whatsoever after the publication of ARRIVE-2010.

Subitems 9a, 9b and 9c, focusing on details of housing, husbandry conditions, and welfare-related assessments, respectively, are most important for the quality of housing life of the laboratory animals, yet they see very low standards of reporting in all research. Subitem 9c (welfare-related assessments), however, underwent a significant increase in quality of reporting only for overall species $(P<0.001)$ and rat and rabbit sub-groups after ARRIVE-2010 published ( $P=0.042$ for rats, $P=0.005$ for rabbits). More specifically, for this subitem, the rate of score 2 (approximating fully reported) for post-ARRIVE increased significantly for overall species and rat and rabbit subgroups.

Items 10 - 13: sample size, allocating animals to experimental groups, experimental outcomes, and statistical methods

The following few items demonstrate details given the validity and power of research, which could demonstrate whether researchers have thought, or haven't thought, about how they want their research to be built and performed in order to achieve their desired results. Details of allocations, including randomization methods (subitem 11a) and treatment orders in different experimental groups (subitem 11b), show low standards of reporting of how the allocation of the animals has been performed, something of high importance in clinical research. Surprisingly, subitem $11 \mathrm{~b}$ declined significantly in the quality of reporting (all species: pre-ARRIVE: $17 \%$; post-ARRIVE: $6 \% ; P=0.006$ ).

Among all articles, experimental outcomes were reported very well (item 12, 100\%). Statistical methods used were commonly described as well (subitem 13a, 92\% to 94\%), however, the units of analysis for each data set weren't clear at all (subitem 13b, 1\%). Besides mentioning the statistical methods used, reporting the assumptions needed to 
apply those statistical tests (subitem 13c) increased significantly since the publication of the ARRIVE-2010 guidelines for rabbit studies (pre-ARRIVE: 10\%; post-ARRIVE: $32 \% ; P=0.034)$. However, no significant difference was found in the overall species and other sub-groups.

Items 14 - 17: Baseline data, numbers analyzed, outcomes and estimation, and adverse events

The following four items deal with inclusion and exclusion of animals, the health status of animals before treatment, and discrepancies between the number of animals used for research and the number of animals actually included in the data analysis. With most studies receiving a score for not reported at all for item 14 (baseline data) and fewer than $60 \%$ reporting subitem 15 a (numbers analysed in each group) for all species, these items/subitems deserve a higher standard for reporting in preclinical research. Item 16 , which purely describes the measures of precision used (such as SD, SEM, 95\%CI), was reported in 70 to $79 \%$ of articles. The adequate reporting of all adverse events concerning animals is the subject of subitem $17 \mathrm{a}$, which has been reported in 74 to $79 \%$ of all the included animal studies.

\section{Item 18 - 20: Interpretation, limitation, translation and funding}

While articles almost always interpret their results (subitem 18a, 100\%), only around half of those mention limitations that occurred during their research (subitem 18b, 50 to $63 \%$ ). There were significant improvements for post-ARRIVE mentioned limitations for overall species $(P=0.036)$ and rabbit studies $(P=0.001)$. Results don't always lead to translation into other species or human biology (item 19), which contrasts result interpretation (subitem 18a). Results in only 44 to $53 \%$ of articles were translated based on generalizability or clinical relevancy, while $100 \%$ of articles interpreted their results. Lastly, item 20 describing funding details displayed significant difference between pre- and post-ARRIVE for overall species, as well as all sub-groups of animals (rats, $P<0.001$; Rabbits, $P<0.001$; Pigs, $P<0.001$ ). However, the rate of articles fully reported for funding details increased only in rat and rabbit studies.

\section{Discussion}

Animal studies remain of importance in current scientific studies and more than 12 million animals have been used for experiments in Europe alone in 2005. ${ }^{24}$ ARRIVE guidelines have been developed to improve the reporting quality of manuscripts, intended to reduce unnecessary animal studies. ${ }^{25}$ Although ARRIVE guidelines were published as early as 2010, no previous studies have evaluated the effect of ARRIVE guidelines on reporting quality, concerning animal hernia models repaired with mesh. 
The present study has systematically searched articles and compared all the applicable ARRIVE items between articles published five years before ARRIVE-2010 and articles published within five years until ARRIVE-2020, evaluating all species together and separately. We found that the quantity of the published articles increased a lot while the quality of reporting only improved on limited items of the ARRIVE-2010 guidelines. The mean number of fully reported (sub)items per article has significantly increased to 14 out of 35 (sub)items after ARRIVE-2010, with a large number of items remaining that should be improved further. Only 8 out of 21 (sub)items have more than half rate of articles fully reported in the post-2019 group for the most recent articles published last year, leaving room for further improving.

According to our results, only several items have been reported well after ARRIVE was published, indicating that there is still room for improvement in the future. The scientific background (subitem 3a), the aim (item 4), the experimental outcomes (item 12 ), and the interpretation of results (subitem 18a), have been reported in virtually all articles of pre-ARRIVE and post-ARRIVE. Therefore, the ARRIVE-2010 guidelines had no effect on these (sub)items. Similarly, the number of experimental groups (subitem 6a) and the details of statistical methods (subitem 13a) have been reported well in more than $90 \%$ articles for both pre-ARRIVE and post-ARRIVE for overall species. These subitems are reported not only for animal experiments but also for most other scientific papers ${ }^{26}$ and may be the more well-known standards among researchers. Subitem $11 \mathrm{~b}$ (the order for different treatments) has shown significant difference for overall species between pre-ARRIVE and post-ARRIVE articles. However, individually in the rat studies, the rabbit studies, or the pig studies, no significant differences or improvements were found regarding $11 \mathrm{~b}$ (the order for different treatments). Similarly, in spite of significant difference for (sub)item 6a (the number of groups) in pig studies, 13c (assumptions of statitistical methods) in rabbit studies, and 18c (three Rs) in rabbit studies, no significant differences for these (sub)items were found for the overall species. This might be explained by the advantage of having a larger sample size in the overall group, compared to the smaller groups evaluating separate species. In contrast, in all the three species, significant differences on item 20 (funding) were found separately of each other, as well as overall species.

Both higher restrictions and enforcing ARRIVE guidelines on being published in journals might have positive effects on the improvement of these items. Vivian et al, comparing the reporting quality of articles between journals supporting ARRIVE and journals not supporting ARRIVE, have illustrated that no significant difference was found between journals. ${ }^{27}$ Therefore, the improvement of these items could be caused by more reporters being aware of ARRIVE guidelines. However, one subitem (subitem $11 \mathrm{~b}$, allocating animals to groups) for overall species and one item (item 1, title 
information) for rabbit studies showed the degraded reporting quality (score of fully reported), making the ARRIVE-2010 effect on reporting quality complex.

From our results, ARRIVE-2010 guidelines might have imbalanced effects of improving reporting quality on different species. The numbers of (sub)items with significant significance between pre- and post-ARRIVE were three for rat studies, eight for rabbit species, and three for pig studies, indicating the imbalanced effect of ARRIVE on the reporting quality for different species.

Two possible factors influencing the reporting quality of articles need to be noted. Firstly, ARRIVE guidelines are usually used as a checklist for manuscripts to ensure the maximization of revealing the essential details of animal experiments. However, it would be better to comply with a combination of ARRIVE guidelines and PREPARE guidelines, the latter which are checked before the animal experiments actually start. Due to a large number of details needed to be reported and much information needed to be recorded during the animal experiments for the ARRIVE guidelines, details could be missed or ignored. Furthermore, in case that items reported by ARRIVE are not included in the experiment, reporting on them could become more difficult for authors. Secondly, chances are that a lot of articles do not report on some items because these are considered to be obvious. For example, some animal experiments using isoflurane as anesthesia did not mention the dose or the method of its administration, because inhalation of isoflurane is the only method of administration for isoflurane. Most animal experiments did not mention the experimental units but could be deduced from the reporting. It would be better to report these details clearly to avoid any uncertainties and maximize reproducibility.

The ARRIVE guidelines have received wide support from the scientific community. Several journals have published the updated ARRIVE guidelines, ${ }^{11,28-30}$ which is rare for scientific papers and might be because of its visibility and the urge to reach the entire scientific population. Furthermore, since the first publication of the ARRIVE guidelines in 2010, more than a thousand journals endorsed the ARRIVE in their guidance for authors. ${ }^{11}$ Despite such high-level support, the impact of ARRIVE on animal reporting remains limited. ${ }^{27,31,32}$ This is consistent with our results. In our results, few researchers clearly mentioned the adherence of the ARRIVE guidelines in their reporting even after the updated guidelines released. This may suggest that voluntary guidelines could be ignored by researchers or at least without sufficient awareness of the application of ARRIVE in their reporting for animal experiments. Disregarding the enforcement of application of ARRIVE, promoting the awareness of ARRIVE by animal welfare body before the design of an animal experiment, instead of after the completion, could help increase the appreciation of authors to adhere to ARRIVE. 
There might be some limitations to this study. The scoring of reporting quality that is reliant on the observers could produce bias. However, in this study, two researchers evaluated all of these independently. Afterwards, all the discrepancies were discussed on each (sub)item of each included article, presenting double-checked results for the following statistical analysis. This should have minimized the observer bias on reporting quality. Additionally, the strict scoring criteria in this study embodied more details than normally, so that deviating from the scoring criteria and judging subjectively was nigh impossible. Nevertheless, in consideration of the limited improvements of reporting after the ARRIVE-2010 guidelines, it would be better to alert animal experiment reporters to maximize the relevant information reported on their manuscripts.

In summary, limited items have been improved after the publication of ARRIVE-2010. Imbalanced improvements in reporting quality exist in different species. The mean numbers of fully reported (sub)items per article have been improved significantly but more improvements are required. Although these results derived from articles that focused on abdominal wall hernia repair with mesh placement, it can be assumed that this is also the case for other animal studies. Further studies and actions concerning (how to improve) the application and awareness of ARRIVE guidelines in animal experiments are necessary, especially after ARRIVE-2.0 has already been published.

\section{Conclusion}

The reporting quality regarding abdominal wall hernia repair in animal experiments has been improved much after the publication of ARRIVE-2010. However, a big gap remains with respect to full reporting for all articles using the checklists in both ARRIVE-2010 and ARRIVE-2020, requiring more efforts to promote ARRIVE adherence for animal experiments in the future. 


\section{References}

1. Ellis H. The first successful elective laparotomy. J Perioper Pract. 2008;18(5):211.

2. Centraal Bureau voor de Statistiek. Operaties in het ziekenhuis; soort opname, leeftijd en geslacht,1995-2010. CBS 2014.

3. Bonjer HJ, Bruining HA. Surgery in the Netherlands. Arch Surg. 1999;134(1):92-8.

4. Kossler-Ebs JB, Grummich K, Jensen K, Huttner FJ, Muller-Stich B, Seiler CM, et al. Incisional Hernia Rates After Laparoscopic or Open Abdominal Surgery-A Systematic Review and MetaAnalysis. World J Surg. 2016;40(10):2319-30.

5. Ellis H. Internal overhealing: the problem of intraperitoneal adhesions. World J Surg. 1980;4(3):303-6.

6. Duron JJ. Postoperative intraperitoneal adhesion pathophysiology. Colorectal Dis. 2007;9 Suppl 2: 14-24.

7. Krafts KP. Tissue repair: The hidden drama. Organogenesis. 2010;6(4):225-33.

8. Dubay DA, Wang X, Kuhn MA, Robson MC, Franz MG. The prevention of incisional hernia formation using a delayed-release polymer of basic fibroblast growth factor. Ann Surg. 2004;240(1):179-86.

9. Bordoni B, Mahabadi N, Varacallo M. Anatomy, Fascia. StatPearls. Treasure Island (FL)2020.

10. Sanders DL, Kingsnorth AN. The modern management of incisional hernias. BMJ. 2012;344:e2843.

11. Bosanquet DC, Ansell J, Abdelrahman T, Cornish J, Harries R, Stimpson A, et al. Systematic Review and Meta-Regression of Factors Affecting Midline Incisional Hernia Rates: Analysis of 14,618 Patients. PLoS One. 2015;10(9):e0138745.

12. Fink C, Baumann $\mathrm{P}$, Wente MN, Knebel P, Bruckner T, Ulrich A, et al. Incisional hernia rate 3 years after midline laparotomy. Br J Surg. 2014;101(2):51-4.

13. Gillion JF, Sanders D, Miserez M, Muysoms F. The economic burden of incisional ventral hernia repair: a multicentric cost analysis. Hernia. 2016;20(6):819-30.

14. Poulose BK, Shelton J, Phillips S, Moore D, Nealon W, Penson D, et al. Epidemiology and cost of ventral hernia repair: making the case for hernia research. Hernia. 2012;16(2):179-83.

15. Pollock AV, Evans M. Early prediction of late incisional hernias. Br J Surg. 1989;76(9):953-4.

16. Thankam FG, Palanikumar G, Fitzgibbons RJ, Agrawal DK. Molecular Mechanisms and Potential Therapeutic Targets in Incisional Hernia. J Surg Res. 2019;236:134-43.

17. Bingöl D, Ozkaya O, Oymak MO. Surgical Anatomy of the Abdominal Wall. Springer, Cham.; 2017.

18. Caglia P, Tracia A, Borzi L, Amodeo L, Tracia L, Veroux M, et al. Incisional hernia in the elderly: risk factors and clinical considerations. Int J Surg. 2014;12 Suppl 2:S164-S9.

19. Basta MN, Kozak GM, Broach RB, Messa CAt, Rhemtulla I, DeMatteo RP, et al. Can We Predict Incisional Hernia?: Development of a Surgery-specific Decision-Support Interface. Ann Surg. 2019;270(3):544-53.

20. Mommers EHH, van Kooten L, Nienhuijs SW, de Vries Reilingh TS, Lubbers T, Mees BME, et al. Can Electric Nose Breath Analysis Identify Abdominal Wall Hernia Recurrence and Aortic Aneurysms? A Proof-of-Concept Study. Surg Innov. 2020:1553350620917898.

21. Velnar T, Bailey T, Smrkolj V. The wound healing process: an overview of the cellular and molecular mechanisms. J Int Med Res. 2009;37(5):1528-42.

22. Muysoms FE, Antoniou SA, Bury K, Campanelli G, Conze J, Cuccurullo D, et al. European Hernia Society guidelines on the closure of abdominal wall incisions. Hernia. 2015;19(1):1-24.

23. Franz MG, Smith PD, Wachtel TL, Wright TE, Kuhn MA, Ko F, et al. Fascial incisions heal faster than skin: a new model of abdominal wall repair. Surgery. 2001;129(2):203-8.

24. Dubay DA, Wang X, Kirk S, Adamson B, Robson MC, Franz MG. Fascial fibroblast kinetic activity is increased during abdominal wall repair compared to dermal fibroblasts. Wound Repair Regen. 2004;12(5):539-45.

25. Douglas DM. The healing of aponeurotic incisions. Br J Surg. 1952;40(159):79-84.

26. Lemonnier F, Gautier M, Wolfrom C, Lemonnier A. Some metabolic differences between human skin and aponeurosis fibroblasts in culture. J Cell Physiol. 1980;104(3):415-23. 
27. Jansen PL, Mertens Pr P, Klinge U, Schumpelick V. The biology of hernia formation. Surgery. 2004;136(1):1-4.

28. Franz MG. The biology of hernia formation. Surg Clin North Am. 2008;88(1):1-15, vii.

29. Vogels RRM, Kaufmann R, van den Hil LCL, van Steensel S, Schreinemacher MHF, Lange JF, et al. Critical overview of all available animal models for abdominal wall hernia research. Hernia. 2017;21(5):667-75.

30. Wijesinghe DS, Warncke UO, Diegelmann RF. Human as the Ultimate Wound Healing Model: Strategies for Studies Investigating the Dermal Lipidome. Curr Dermatol Rep. 2016;5(4):244-51.

31. Vaughan EM, You JS, Elsie Yu HY, Lasek A, Vitale N, Hornberger TA, et al. Lipid domain-dependent regulation of single-cell wound repair. Mol Biol Cell. 2014;25(12):1867-76.

32. Demoyer JS, Skalak TC, Durieux ME. Lysophosphatidic acid enhances healing of acute cutaneous wounds in the mouse. Wound Repair Regen. 2000;8(6):530-7.

33. Oh SY, Lee SJ, Jung YH, Lee HJ, Han HJ. Arachidonic acid promotes skin wound healing through induction of human MSC migration by MT3-MMP-mediated fibronectin degradation. Cell Death Dis. 2015;6:e1750.

34. Dhall S, Wijesinghe DS, Karim ZA, Castro A, Vemana HP, Khasawneh FT, et al. Arachidonic acidderived signaling lipids and functions in impaired healing. Wound Repair Regen. 2015;23(5):644-56.

35. Bruggmann D, Tchartchian G, Wallwiener M, Munstedt K, Tinneberg HR, Hackethal A. Intraabdominal adhesions: definition, origin, significance in surgical practice, and treatment options. Dtsch Arztebl Int. 2010;107(44):769-75.

36. Tabibian N, Swehli E, Boyd A, Umbreen A, Tabibian JH. Abdominal adhesions: A practical review of an often overlooked entity. Ann Med Surg (Lond). 2017;15:9-13.

37. Stommel MWJ, Ten Broek RPG, Strik C, Slooter GD, Verhoef C, Grunhagen DJ, et al. Multicenter Observational Study of Adhesion Formation After Open-and Laparoscopic Surgery for Colorectal Cancer. Ann Surg. 2018;267(4):743-8.

38. ten Broek RP, Issa Y, van Santbrink EJ, Bouvy ND, Kruitwagen RF, Jeekel J, et al. Burden of adhesions in abdominal and pelvic surgery: systematic review and met-analysis. BMJ. 2013;347:f5588.

39. Maciver AH, McCall M, James Shapiro AM. Intra-abdominal adhesions: cellular mechanisms and strategies for prevention. Int J Surg. 2011;9(8):589-94.

40. Holmdahl L. The role of fibrinolysis in adhesion formation. Eur J Surg Suppl. 1997(577):24-31. 405

41. Boland GM, Weigel RJ. Formation and prevention of postoperative abdominal adhesions. J Surg Res. 2006;132(1):3-12.

42. Arung W, Meurisse M, Detry O. Pathophysiology and prevention of postoperative peritoneal adhesions. World J Gastroenterol. 2011;17(41):4545-53.

43. Ward BC, Panitch A. Abdominal adhesions: current and novel therapies. J Surg Res. 2011;165(1): 91-111.

44. Jairam AP, Timmermans L, Eker HH, Pierik R, van Klaveren D, Steyerberg EW, et al. Prevention of incisional hernia with prophylactic onlay and sublay mesh reinforcement versus primary suture only in midline laparotomies (PRIMA): 2-year follow-up of a multicentre, double-blind, randomised controlled trial. Lancet. 2017;390(10094):567-76.

45. Moreno-Egea A, Bustos JA, Girela E, Aguayo-Albasini JL. Long-term results of laparoscopic repair of incisional hernias using an intraperitoneal composite mesh. Surg Endosc. 2010;24(2):359-65.

46. van den Hil LCL, Vogels RRM, van Barneveld KWY, Gijbels MJJ, Peutz-Kootstra CJ, Cleutjens JPM, et al. Comparability of histological outcomes in rats and humans in a hernia model. J Surg Res. 2018;229:271-6.

47. Navaratnam AV, Ariyaratnam R, Smart NJ, Parker M, Motson RW, Arulampalam TH. Incisional hernia rate after laparoscopic colorectal resection is reduced with standardisation of specimen extraction. Ann R Coll Surg Engl. 2015;97(1):17-21.

48. Lee L, Mata J, Droeser RA, Kaneva P, Liberman S, Charlebois P, et al. Incisional Hernia After Midline Versus Transverse Specimen Extraction Incision: A Randomized Trial in Patients Undergoing Laparoscopic Colectomy. Ann Surg. 2018;268(1):41-7. 
49. Braga M, Frasson M, Vignali A, Zuliani W, Civelli V, Di Carlo V. Laparoscopic vs. open colectomy in cancer patients: long-term complications, quality of life, and survival. Dis Colon Rectum. 2005; 48(12):2217-23.

50. Ihedioha U, Mackay G, Leung E, Molloy RG, O'Dwyer PJ. Laparoscopic colorectal resection does not reduce incisional hernia rates when compared with open colorectal resection. Surg Endosc. 2008; 22(3):689-92.

51. Whang SH, Astudillo JA, Sporn E, Bachman SL, Miedema BW, Davis W, et al. In search of the best peritoneal adhesion model: comparison of different techniques in a rat model. J Surg Res. 2011; 167(2):245-50.

52. Rajab TK, Wauschkuhn CA, Smaxwil L, Kraemer B, Wallwiener M, Wallwiener CW. An improved model for the induction of experimental adhesions. J Invest Surg. 2010;23(1):35-9.

53. Miller LR, Marks C, Becker JB, Hurn PD, Chen WJ, Woodruff T, et al. Considering sex as a biological variable in preclinical research. FASEB J. 2017;31(1):29-34.

54. Franco NH. Animal Experiments in Biomedical Research: A Historical Perspective. Animals (Basel). 2013;3(1):238-73.

55. Doke SK, Dhawale SC. Alternatives to animal testing: A review. Saudi Pharm J. 2015;23(3):223-9.

56. Combes RD, Berridge T, Connelly J, Eve MD, Garner RC, Toon S, et al. Early microdose drug studies in human volunteers can minimise animal testing: Proceedings of a workshop organised by Volunteers in Research and Testing. Eur J Pharm Sci. 2003;19(1):1-11.

57. Taylor K, Alvarez LR. An Estimate of the Number of Animals Used for Scientific Purposes Worldwide in 2015. Altern Lab Anim. 2019;47(5-6):196-213.

58. Bringman S, Conze J, Cuccurullo D, Deprest J, Junge K, Klosterhalfen B, et al. Hernia repair: the search for ideal meshes. Hernia. 2010;14(1):81-7.

59. Baylon K, Rodriguez-Camarillo P, Elias-Zuniga A, Diaz-Elizondo JA, Gilkerson R, Lozano K. Past, Present and Future of Surgical Meshes: A Review. Membranes (Basel). 2017;7(3). 


\section{Supplemental material}

Table S2.1 Full searching strategies updated on 21st July, 2020

a

\section{Pubmed}

((mesh*[tw] OR prothes*[tw] OR prosthet*[tw]) AND (herni*[tiab] OR hernia[mesh:noexp] OR Hernia, Abdominal[mesh] OR herniorrhaphy[mesh])) AND (("animal experimentation"[MeSH Terms] OR "models, animal"[MeSH Terms] OR "invertebrates"[MeSH Terms] OR "Animals"[Mesh:noexp] OR "animal population groups"[MeSH Terms] OR "chordata"[MeSH Terms:noexp] OR "chordata, nonvertebrate"[MeSH Terms] OR "vertebrates"[MeSH Terms:noexp] OR "amphibians"[MeSH Terms] OR "birds"[MeSH Terms] OR "fishes"[MeSH Terms] OR "reptiles"[MeSH Terms] OR "mammals"[MeSH Terms:noexp] OR "primates"[MeSH Terms:noexp] OR "artiodactyla"[MeSH Terms] OR "carnivora"[MeSH Terms] OR "cetacea"[MeSH Terms] OR "chiroptera"[MeSH Terms] OR "elephants"[MeSH Terms] OR "hyraxes"[MeSH Terms] OR "insectivora"[MeSH Terms] OR "lagomorpha"[MeSH Terms] OR "marsupialia"[MeSH Terms] OR "monotremata"[MeSH Terms] OR "perissodactyla"[MeSH Terms] OR "rodentia"[MeSH Terms] OR "scandentia"[MeSH Terms] OR "sirenia"[MeSH Terms] OR "xenarthra"[MeSH Terms] OR "haplorhini"[MeSH Terms:noexp] OR "strepsirhini"[MeSH Terms] OR "platyrrhini"[MeSH Terms] OR "tarsii"[MeSH Terms] OR "catarrhini"[MeSH Terms:noexp] OR "cercopithecidae"[MeSH Terms] OR "hylobatidae"[MeSH Terms] OR "hominidae"[MeSH Terms:noexp] OR "gorilla gorilla"[MeSH Terms] OR "pan paniscus"[MeSH Terms] OR "pan troglodytes"[MeSH Terms] OR "pongo pygmaeus"[MeSH Terms]) OR ((animals[tiab] OR animal[tiab] OR mice[Tiab] OR mus[Tiab] OR mouse[Tiab] OR murine[Tiab] OR woodmouse[tiab] OR rats[Tiab] OR rat[Tiab] OR murinae[Tiab] OR muridae[Tiab] OR cottonrat[tiab] OR cottonrats[tiab] OR hamster[tiab] OR hamsters[tiab] OR cricetinae[tiab] OR rodentia[Tiab] OR rodent[Tiab] OR rodents[Tiab] OR pigs[Tiab] OR pig[Tiab] OR swine[tiab] OR swines[tiab] OR piglets[tiab] OR piglet[tiab] OR boar[tiab] OR boars[tiab] OR "sus scrofa"[tiab] OR ferrets[tiab] OR ferret[tiab] OR polecat[tiab] OR polecats[tiab] OR "mustela putorius"[tiab] OR "guinea pigs"[Tiab] OR "guinea pig"[Tiab] OR cavia[Tiab] OR callithrix[Tiab] OR marmoset[Tiab] OR marmosets[Tiab] OR cebuella[Tiab] OR hapale[Tiab] OR octodon[Tiab] OR chinchilla[Tiab] OR chinchillas[Tiab] OR gerbillinae[Tiab] OR gerbil[Tiab] OR gerbils[Tiab] OR jird[Tiab] OR jirds[Tiab] OR merione[Tiab] OR meriones[Tiab] OR rabbits[Tiab] OR rabbit[Tiab] OR hares[Tiab] OR hare[Tiab] OR diptera[Tiab] OR flies[Tiab] OR fly[Tiab] OR dipteral[Tiab] OR drosphila[Tiab] OR drosophilidae[Tiab] OR cats[Tiab] OR cat[Tiab] OR carus[Tiab] OR felis[Tiab] OR nematoda[Tiab] OR nematode[Tiab] OR nematoda[Tiab] OR nematode[Tiab] OR nematodes[Tiab] OR sipunculida[Tiab] OR dogs[Tiab] OR dog[Tiab] OR canine[Tiab] OR canines[Tiab] OR canis[Tiab] OR sheep[Tiab] OR sheeps[Tiab] OR mouflon[Tiab] OR mouflons[Tiab] OR ovis[Tiab] OR goats[Tiab] OR goat[Tiab] OR capra[Tiab] OR capras[Tiab] OR rupicapra[Tiab] OR chamois[Tiab] OR haplorhini[Tiab] OR monkey[Tiab] OR monkeys[Tiab] OR anthropoidea[Tiab] OR anthropoids[Tiab] OR saguinus[Tiab] OR tamarin[Tiab] OR tamarins[Tiab] OR leontopithecus[Tiab] OR hominidae[Tiab] OR ape[Tiab] OR apes[Tiab] OR pan[Tiab] OR paniscus[Tiab] OR "pan paniscus"[Tiab] OR bonobo[Tiab] OR bonobos[Tiab] OR troglodytes[Tiab] OR "pan troglodytes"[Tiab] OR gibbon[Tiab] OR gibbons[Tiab] OR siamang[Tiab] OR siamangs[Tiab] OR nomascus[Tiab] OR symphalangus[Tiab] OR chimpanzee[Tiab] OR chimpanzees[Tiab] OR prosimians[Tiab] OR "bush baby"[Tiab] OR prosimian[Tiab] OR bush babies[Tiab] OR galagos[Tiab] OR galago[Tiab] OR pongidae[Tiab] OR gorilla[Tiab] OR gorillas[Tiab] OR pongo[Tiab] OR pygmaeus[Tiab] OR "pongo pygmaeus"[Tiab] OR orangutans[Tiab] OR pygmaeus[Tiab] OR lemur[Tiab] OR lemurs[Tiab] OR lemuridae[Tiab] OR horse[Tiab] OR horses[Tiab] OR pongo[Tiab] OR equus[Tiab] OR cow[Tiab] OR calf[Tiab] OR bull[Tiab] OR chicken[Tiab] OR chickens[Tiab] OR gallus[Tiab] OR quail[Tiab] OR bird[Tiab] OR birds[Tiab] OR quails[Tiab] OR poultry[Tiab] OR poultries[Tiab] OR fowl[Tiab] OR fowls[Tiab] OR reptile[Tiab] OR reptilia[Tiab] OR reptiles[Tiab] OR snakes[Tiab] OR snake[Tiab] OR lizard[Tiab] OR lizards[Tiab] OR alligator[Tiab] OR alligators[Tiab] OR crocodile[Tiab] OR crocodiles[Tiab] OR turtle[Tiab] OR turtles[Tiab] OR amphibian[Tiab] OR amphibians[Tiab] OR amphibia[Tiab] OR frog[Tiab] OR frogs[Tiab] OR bombina[Tiab] OR salientia[Tiab] OR toad[Tiab] OR toads[Tiab] OR "epidalea calamita"[Tiab] OR salamander[Tiab] OR salamanders[Tiab] OR eel[Tiab] OR eels[Tiab] OR fish[Tiab] OR fishes[Tiab] OR pisces[Tiab] OR catfish[Tiab] OR catfishes[Tiab] OR : arius[Tiab] OR heteropneustes[Tiab] OR sheatfish[Tiab] OR perch[Tiab] OR 
perches[Tiab] OR percidae[Tiab] OR perca[Tiab] OR trout[Tiab] OR trouts[Tiab] OR char[Tiab] OR chars[Tiab] OR salvelinus[Tiab] OR "fathead minnow"[Tiab] OR minnow[Tiab] OR cyprinidae[Tiab] OR carps[Tiab] OR carp[Tiab] OR zebrafish[Tiab] OR zebrafishes[Tiab] OR goldfish[Tiab] OR goldfishes[Tiab] OR guppy[Tiab] OR guppies[Tiab] OR chub[Tiab] OR chubs[Tiab] OR tinca[Tiab] OR barbels[Tiab] OR barbus[Tiab] OR pimephales[Tiab] OR promelas[Tiab] OR "poecilia reticulata"[Tiab] OR mullet[Tiab] OR mullets[Tiab] OR seahorse[Tiab] OR seahorses[Tiab] OR mugil curema[Tiab] OR atlantic cod[Tiab] OR shark[Tiab] OR sharks[Tiab] OR catshark[Tiab] OR anguilla[Tiab] OR salmonid[Tiab] OR salmonids[Tiab] OR whitefish[Tiab] OR whitefishes[Tiab] OR salmon[Tiab] OR salmons[Tiab] OR sole[Tiab] OR solea[Tiab] OR "sea lamprey"[Tiab] OR lamprey[Tiab] OR lampreys[Tiab] OR pumpkinseed[Tiab] OR sunfish[Tiab] OR sunfishes[Tiab] OR tilapia[Tiab] OR tilapias[Tiab] OR turbot[Tiab] OR turbots[Tiab] OR flatfish[Tiab] OR flatfishes[Tiab] OR sciuridae[Tiab] OR squirrel[Tiab] OR squirrels[Tiab] OR chipmunk[Tiab] OR chipmunks[Tiab] OR suslik[Tiab] OR susliks[Tiab] OR vole[Tiab] OR voles[Tiab] OR lemming[Tiab] OR lemmings[Tiab] OR muskrat[Tiab] OR muskrats[Tiab] OR lemmus[Tiab] OR otter[Tiab] OR otters[Tiab] OR marten[Tiab] OR martens[Tiab] OR martes[Tiab] OR weasel[Tiab] OR badger[Tiab] OR badgers[Tiab] OR ermine[Tiab] OR mink[Tiab] OR minks[Tiab] OR sable[Tiab] OR sables[Tiab] OR gulo[Tiab] OR gulos[Tiab] OR wolverine[Tiab] OR wolverines[Tiab] OR minks[Tiab] OR mustela[Tiab] OR llama[Tiab] OR llamas[Tiab] OR alpaca[Tiab] OR alpacas[Tiab] OR camelid[Tiab] OR camelids[Tiab] OR guanaco[Tiab] OR guanacos[Tiab] OR chiroptera[Tiab] OR chiropteras[Tiab] OR bat[Tiab] OR bats[Tiab] OR fox[Tiab] OR foxes[Tiab] OR iguana[Tiab] OR iguanas[Tiab] OR xenopus laevis[Tiab] OR parakeet[Tiab] OR parakeets[Tiab] OR parrot[Tiab] OR parrots[Tiab] OR donkey[Tiab] OR donkeys[Tiab] OR mule[Tiab] OR mules[Tiab] OR zebra[Tiab] OR zebras[Tiab] OR shrew[Tiab] OR shrews[Tiab] OR bison[Tiab] OR bisons[Tiab] OR buffalo[Tiab] OR buffaloes[Tiab] OR deer[Tiab] OR deers[Tiab] OR bear[Tiab] OR bears[Tiab] OR panda[Tiab] OR pandas[Tiab] OR "wild hog"[Tiab] OR "wild boar"[Tiab] OR fitchew[Tiab] OR fitch[Tiab] OR beaver[Tiab] OR beavers[Tiab] OR jerboa[Tiab] OR jerboas[Tiab] OR capybara[Tiab] OR capybaras[Tiab]) NOT medline[subset]))

b

Embase

1 surgical equipment/ or mesh*.ab,ti. or prothes*.ab,ti. or prosthet*.ab,ti.

2 herni*.ab,ti. or hernioplasty/ or herniorrhaphy/ or herniotomy/ or hernia/ or exp abdominal wall hernia/ or incisional hernia/

3 exp animal experiment/ or exp animal model/ or exp experimental animal/ or exp transgenic animal/ or exp male animal/ or exp female animal/ or exp juvenile animal/ or animal/ or chordata/ or vertebrate/ or tetrapod/or exp fish/ or amniote/ or exp amphibia/ or mammal/or exp reptile/ or exp sauropsid/ or therian/ or exp monotremate/ or placental mammals/ or exp marsupial/ or Euarchontoglires/ or exp Afrotheria/ or exp Boreoeutheria/ or exp Laurasiatheria/ or exp Xenarthra/ or primate/ or exp Dermoptera/ or exp Glires/ or exp Scandentia/ or Haplorhini/ or exp prosimian/ or simian/ or exp tarsiiform/ or Catarrhini/ or exp Platyrrhini/ or ape/ or exp Cercopithecidae/ or hominid/ or exp hylobatidae/ or exp chimpanzee/ or exp gorilla/ or exp orang utan/ or (animal or animals or pisces or fish or fishes or catfish or catfishes or sheatfish or silurus or arius or heteropneustes or clarias or gariepinus or fathead minnow or fathead minnows or pimephales or promelas or cichlidae or trout or trouts or char or chars or salvelinus or salmo or oncorhynchus or guppy or guppies or millionfish or poecilia or goldfish or goldfishes or carassius or auratus or mullet or mullets or mugil or curema or shark or sharks or cod or cods or gadus or morhua or carp or carps or cyprinus or carpio or killifish or eel or eels or anguilla or zander or sander or lucioperca or stizostedion or turbot or turbots or psetta or flatfish or flatfishes or plaice or pleuronectes or platessa or tilapia or tilapias or oreochromis or sarotherodon or common sole or dover sole or solea or zebrafish or zebrafishes or danio or rerio or seabass or dicentrarchus or labrax or morone or lamprey or lampreys or petromyzon or pumpkinseed or pumpkinseeds or lepomis or gibbosus or herring or clupea or harengus or amphibia or amphibian or amphibians or anura or salientia or frog or frogs or rana or toad or toads or bufo or xenopus or laevis or bombina or epidalea or calamita or salamander or salamanders or newt or newts or triturus or reptilia or reptile or reptiles or bearded dragon or pogona or vitticeps or iguana or iguanas or lizard or lizards or anguis fragilis or turtle or turtles or snakes or snake or aves or bird or birds or quail or quails or coturnix 
or bobwhite or colinus or virginianus or poultry or poultries or fowl or fowls or chicken or chickens or gallus or zebra finch or taeniopygia or guttata or canary or canaries or serinus or canaria or parakeet or parakeets or grasskeet or parrot or parrots or psittacine or psittacines or shelduck or tadorna or goose or geese or branta or leucopsis or woodlark or lullula or flycatcher or ficedula or hypoleuca or dove or doves or geopelia or cuneata or duck or ducks or greylag or graylag or anser or harrier or circus pygargus or red knot or great knot or calidris or canutus or godwit or limosa or lapponica or meleagris or gallopavo or jackdaw or corvus or monedula or ruff or philomachus or pugnax or lapwing or peewit or plover or vanellus or swan or cygnus or columbianus or bewickii or gull or chroicocephalus or ridibundus or albifrons or great tit or parus or aythya or fuligula or streptopelia or risoria or spoonbill or platalea or leucorodia or blackbird or turdus or merula or blue tit or cyanistes or pigeon or pigeons or columba or pintail or anas or starling or sturnus or owl or athene noctua or pochard or ferina or cockatiel or nymphicus or hollandicus or skylark or alauda or tern or sterna or teal or crecca or oystercatcher or haematopus or ostralegus or shrew or shrews or sorex or araneus or crocidura or russula or european mole or talpa or chiroptera or bat or bats or eptesicus or serotinus or myotis or dasycneme or daubentonii or pipistrelle or pipistrellus or cat or cats or felis or catus or feline or dog or dogs or canis or canine or canines or otter or otters or lutra or badger or badgers or meles or fitchew or fitch or foumart or foulmart or ferrets or ferret or polecat or polecats or mustela or putorius or weasel or weasels or fox or foxes or vulpes or common seal or phoca or vitulina or grey seal or halichoerus or horse or horses or equus or equine or equidae or donkey or donkeys or mule or mules or pig or pigs or swine or swines or hog or hogs or boar or boars or porcine or piglet or piglets or sus or scrofa or llama or llamas or lama or glama or deer or deers or cervus or elaphus or cow or cows or bos taurus or bos indicus or bovine or bull or bulls or cattle or bison or bisons or sheep or sheeps or ovis aries or ovine or lamb or lambs or mouflon or mouflons or goat or goats or capra or caprine or chamois or rupicapra or leporidae or lagomorpha or lagomorph or rabbit or rabbits or oryctolagus or cuniculus or laprine or hares or lepus or rodentia or rodent or rodents or murinae or mouse or mice or mus or musculus or murine or woodmouse or apodemus or rat or rats or rattus or norvegicus or guinea pig or guinea pigs or cavia or porcellus or hamster or hamsters or mesocricetus or cricetulus or cricetus or gerbil or gerbils or jird or jirds or meriones or unguiculatus or jerboa or jerboas or jaculus or chinchilla or chinchillas or beaver or beavers or castor fiber or castor canadensis or sciuridae or squirrel or squirrels or sciurus or chipmunk or chipmunks or marmot or marmots or marmota or suslik or susliks or spermophilus or cynomys or cottonrat or cottonrats or sigmodon or vole or voles or microtus or myodes or glareolus or primate or primates or prosimian or prosimians or lemur or lemurs or lemuridae or loris or bush baby or bush babies or bushbaby or bushbabies or galago or galagos or anthropoidea or anthropoids or simian or simians or monkey or monkeys or marmoset or marmosets or callithrix or cebuella or tamarin or tamarins or saguinus or leontopithecus or squirrel monkey or squirrel monkeys or saimiri or night monkey or night monkeys or owl monkey or owl monkeys or douroucoulis or aotus or spider monkey or spider monkeys or ateles or baboon or baboons or papio or rhesus monkey or macaque or macaca or mulatta or cynomolgus or fascicularis or green monkey or green monkeys or chlorocebus or vervet or vervets or pygerythrus or hominoidea or ape or apes or hylobatidae or gibbon or gibbons or siamang or siamangs or nomascus or symphalangus or hominidae or orangutan or orangutans or pongo or chimpanzee or chimpanzees or pan troglodytes or bonobo or bonobos or pan paniscus or gorilla or gorillas or troglodytes).ti,ab.

$4 \quad 1$ and 2 and 3

c

Web of Science

$1 \mathrm{Tl}=$ (mesh* or prothes* or prosthet*) $\mathrm{OR} \mathrm{AB}=$ (mesh* or prothes* or prosthet*) $\mathrm{OR} \mathrm{TS}=$ (surgical equipment or surgical mesh) $\mathrm{OR} \mathrm{KP}=$ (surgical equipment or surgical mesh)

$2 \mathrm{TI}=($ herni*) $\mathrm{OR} \mathrm{AB}=($ herni*) $\mathrm{OR} \mathrm{KP}=$ (hernioplasty or herniorrhaphy or herniotomy or hernia or abdominal wall hernia or incisional hernia)

$3 \mathrm{TS}=$ (animal) OR TS=(nonhuman) OR TI=(animal or animals or rodent or rodents or rat or rats or mice or mouse or hamster or hamsters or rabbit or rabbits or sheep or dog or dogs or goat or goats or pig or pigs or porcine or swine or horse or horses or cow or cows or bovine or bull or bulls or cattle) OR 
$A B=$ (animal or animals or rodent or rodents or rat or rats or mice or mouse or hamster or hamsters or rabbit or rabbits or sheep or dog or dogs or goat or goats or pig or pigs or porcine or swine or horse or horses or cow or cows or bovine or bull or bulls or cattle)

Table S2.2 Scoring criteria

a

Item/subitem in ARRIVE-2010

\begin{tabular}{ll}
\hline $\begin{array}{l}\text { Item } \\
\text { Title } \\
1 \quad \text { Title }\end{array}$ & \\
& $\begin{array}{l}\text { 1. Animal research } \\
\text { 2. If applicable, in vitro or ex vivo } \\
\text { experiment }\end{array}$
\end{tabular}

\section{Score}

All the two items were mentioned, if applicable $=$ Fully reported $=2$

One of the two items was mentioned, if applicable $=$ Partially reported $=1$

None was mentioned $=$ No reported $=0$

\section{Abstract}

2 Abstract

1. Background

2. Aims or hypotheses

3. Animal species or strain

4. Methods

5. Findings

6. Conclusions

All the six items were mentioned $=$ Fully reported $=2$

At least one but less than six items were mentioned $=$ Partially reported $=1$

\section{Introduction}

3a Background

Background information, no need to evaluate the quality of None was mentioned $=$ No reported $=0$

3b Background background

Background information was mentioned $=$ Fully reported $=2$

None was mentioned $=$ No reported $=0$

1. How and why the specific animal The two items were both mentioned $=$ Fully model was chosen reported $=2$

2. Described previously used animal model

Only one of the two items was mentioned $=$ Partially reported $=1$

None was mentioned $=$ No reported $=0$

4 Objective

Clearly mentioned the aims, objectives or hypotheses

The item was mentioned $=$ Fully reported $=2$ None was mentioned $=$ No reported $=0$

\section{Methods}

5 Ethical statement 1. Ethical permission/relevant licences for the use of animals 2. Guidelines of animal experiments (e.g. guidelines, laws, rules, policy, act)

The two items were both mentioned = Fully reported $=2$

Only one of the two items was mentioned $=$ Partially reported $=1$

None was mentioned $=$ No reported $=0$

6a Study design

The number of experimental and control groups

The number was mentioned $=$ Fully reported $=2$

None was mentioned $=$ No reported $=0$

6b Study design

1. Randomization of the allocation

2. Blinding of the allocation

Both the randomization and blinding were mentioned $=$ Fully reported $=2$

3. Optional: If applicable, the reason why randomization and blinding was not used

Explain the reason if applicable $=$ Fully reported $=2$

Only randomization or blinding was mentioned $=$ Partially reported $=1$ None was mentioned $=$ No reported $=0$

6c Study design Experimental units 
Table S2.2 (continued)

\begin{tabular}{|c|c|c|c|}
\hline $7 a$ & $\begin{array}{l}\text { Experimental } \\
\text { procedures }\end{array}$ & $\begin{array}{l}\text { 1. Anaesthesia name, dose, and } \\
\text { route/site ( } 2 \text { or } 3 \text { of these reported } \\
\text { were considered as the mention of } \\
\text { this item) } \\
\text { 2. Analgesia name, dose, and } \\
\text { route/site ( } 2 \text { or } 3 \text { of these reported } \\
\text { were considered as the mention of } \\
\text { this item) } \\
\text { 3. Surgical procedure } \\
\text { 4. Euthanasia (method/name) } \\
\text { 5. Mesh name and mesh suppliers } \\
\text { (only both reported were } \\
\text { considered as the mention of this } \\
\text { item) }\end{array}$ & $\begin{array}{l}\text { All the five items were mentioned }=\text { Fully } \\
\text { reported }=3 \\
\text { Three or four of the five items were } \\
\text { mentioned }=\text { Mostly reported }=2 \\
\text { One or two of the five items were mentioned } \\
=\text { Partially reported }=1 \\
\text { None was mentioned }=\text { No reported }=0\end{array}$ \\
\hline $7 b$ & $\begin{array}{l}\text { Experimental } \\
\text { procedures }\end{array}$ & $\begin{array}{l}\text { Time of day (morning, afternoon, } \\
\text { evening) }\end{array}$ & $\begin{array}{l}\text { The time was mentioned }=\text { Fully reported }=2 \\
\text { None was mentioned }=\text { No reported }=0\end{array}$ \\
\hline $7 \mathrm{c}$ & $\begin{array}{l}\text { Experimental } \\
\text { procedures }\end{array}$ & $\begin{array}{l}\text { The place for operations such as } \\
\text { home cage, laboratory, and water } \\
\text { maze }\end{array}$ & $\begin{array}{l}\text { The place, e.g. standard operation room, was } \\
\text { mentioned }=\text { Fully reported }=2 \\
\text { Reported such as sterile condition or aseptic } \\
\text { environment }=\text { Partially reported }=1 \\
\text { None was mentioned }=\text { No reported }=0\end{array}$ \\
\hline $7 d$ & $\begin{array}{l}\text { Experimental } \\
\text { procedures }\end{array}$ & $\begin{array}{l}\text { 1. The reason why the anaesthetic } \\
\text { was chosen } \\
2 \text {. The reason why the route and } \\
\text { dose of anaesthetic drugs was } \\
\text { chosen }\end{array}$ & $\begin{array}{l}\text { All the two items were mentioned }=\text { Fully } \\
\text { reported }=2 \\
\text { One of the two items was mentioned }= \\
\text { Partially reported }=1 \\
\text { None was mentioned }=\text { No reported }=0\end{array}$ \\
\hline $8 a$ & $\begin{array}{l}\text { Experimental } \\
\text { animals }\end{array}$ & $\begin{array}{l}\text { 1. Species } \\
\text { 2. Strain } \\
\text { 3. Sex } \\
\text { 4. Age } \\
\text { 5. Weight }\end{array}$ & $\begin{array}{l}\text { All the five items were mentioned }=\text { Fully } \\
\text { reported }=3 \\
\text { Three or four of the five items were } \\
\text { mentioned }=\text { Mostly reported }=2 \\
\text { One or two of the five items were mentioned } \\
=\text { Partially reported }=1 \\
\text { None was mentioned }=\text { No reported }=0\end{array}$ \\
\hline $8 b$ & $\begin{array}{l}\text { Experimental } \\
\text { animals }\end{array}$ & $\begin{array}{l}\text { 1. The supplier of the animal } \\
\text { 2. Genetic information, or } \\
\text { nomenclature, or health/immune } \\
\text { status } \\
\text { 3. Previous drugs/procedures, or } \\
\text { health report, or acclimatization }\end{array}$ & $\begin{array}{l}\text { All the three items were mentioned }=\text { Fully } \\
\text { reported }=2 \\
\text { One of the three items was mentioned }= \\
\text { Partially reported }=1 \\
\text { None was mentioned }=\text { No reported }=0\end{array}$ \\
\hline $9 a$ & $\begin{array}{l}\text { Housing and } \\
\text { husbandry }\end{array}$ & $\begin{array}{l}\text { 1. Type of facility } \\
\text { 2. Type of cage } \\
\text { 3. Bedding material } \\
\text { 4. Number of companions }\end{array}$ & $\begin{array}{l}\text { All the four items were mentioned }=\text { Fully } \\
\text { reported }=2 \\
\text { One of the four items was mentioned }= \\
\text { Partially reported }=1 \\
\text { None was mentioned }=\text { No reported }=0\end{array}$ \\
\hline $9 b$ & $\begin{array}{l}\text { Housing and } \\
\text { husbandry }\end{array}$ & $\begin{array}{l}\text { 1. Room temperature } \\
\text { 2. Light/dark cycle } \\
\text { 3. Access to water and food } \\
\text { 4. Type of food } \\
\text { 5. Environmental enrichment }\end{array}$ & $\begin{array}{l}\text { All the five items were mentioned = Fully } \\
\text { reported }=3 \\
\text { Three or four of the five items were } \\
\text { mentioned }=\text { Mostly reported }=2 \\
\text { One or two of the five items were mentioned } \\
=\text { Partially reported }=1 \\
\text { None was mentioned }=\text { No reported }=0\end{array}$ \\
\hline
\end{tabular}


Table S2.2 (continued)

\begin{tabular}{|c|c|c|c|}
\hline $9 \mathrm{c}$ & $\begin{array}{l}\text { Housing and } \\
\text { husbandry }\end{array}$ & $\begin{array}{l}\text { 1. Checking weight or behaviour or } \\
\text { surgical site } \\
\text { 2. If Pain medications were given } \\
\text { or not }\end{array}$ & $\begin{array}{l}\text { The two items were both mentioned }=\text { Fully } \\
\text { reported }=2 \\
\text { Only one of the two items was mentioned }= \\
\text { Partially reported }=1 \\
\text { None was mentioned }=\text { No reported }=0\end{array}$ \\
\hline $10 \mathrm{a}$ & Sample size & $\begin{array}{l}\text { 1. The total number of animals in } \\
\text { each experiment } \\
\text { 2. The number of animals in each } \\
\text { experimental group }\end{array}$ & $\begin{array}{l}\text { The two items were both mentioned }=\text { Fully } \\
\text { reported }=2 \\
\text { Only one of the two items was mentioned }= \\
\text { Partially reported }=1 \\
\text { None was mentioned }=\text { No reported }=0\end{array}$ \\
\hline $10 \mathrm{~b}$ & Sample size & Sample size calculation & $\begin{array}{l}\text { The calculation was mentioned }=\text { Fully } \\
\text { reported }=2 \\
\text { None was mentioned }=\text { No reported }=0\end{array}$ \\
\hline $10 \mathrm{c}$ & Sample size & $\begin{array}{l}\text { Optional item: the number of } \\
\text { replication of experiments if } \\
\text { applicable }\end{array}$ & $\begin{array}{l}\text { The number was mentioned = Fully reported } \\
=2 \\
\text { None was mentioned = No reported }=0 \\
\text { If not applicable, marked as '----' }\end{array}$ \\
\hline $11 \mathrm{a}$ & $\begin{array}{l}\text { Allocating animals } \\
\text { to } \\
\text { experimental }\end{array}$ & $\begin{array}{l}\text { The details of randomization or } \\
\text { allocation to groups }\end{array}$ & $\begin{array}{l}\text { The details were mentioned }=\text { Fully reported } \\
=2 \\
\text { None was mentioned }=\text { No reported }=0\end{array}$ \\
\hline
\end{tabular}

11b Allocating animals Clear order of the allocation to to groups for treatments experimental groups

12 Experimental Primary and secondary outcomes experimental outcomes

13a Statistical methods Statistical methods

13b Statistical methods Unit of analysis for each group

13c Statistical methods Assumptions of statistical method

\section{Results}

14 Baseline data Characteristics and health status of All of the four items were mentioned = Fully animals prior to the treatment

1. Weight

2. Microbiological status

3. Drugs

4. Previous treatments before operations

15a Numbers analysed Report the number of animals in each group with absolute numbers

15b Numbers analysed Optional item:

If applicable, the reason why the animals were excluded

The order was mentioned $=$ Fully reported $=$ 2

None was mentioned $=$ No reported $=0$

The outcomes were mentioned $=$ Fully reported $=2$

None was mentioned $=$ No reported $=0$

The methods were mentioned $=$ Fully reported $=2$

None was mentioned $=$ No reported $=0$ The units were clear $=$ Fully reported $=2$ The units were not clear $=$ No reported $=0$ The assumptions were mentioned $=$ Fully reported $=2$

None was mentioned $=$ No reported $=0$ reported $=2$

One of the four items was mentioned $=$ Partially reported $=1$

None was mentioned $=$ No reported $=0$

The numbers were mentioned $=$ Fully

reported $=2$

None was mentioned $=$ No reported $=0$

The reason was mentioned $=$ Fully reported $=2$

None was mentioned $=$ No reported $=0$

If not applicable, marked as '---' 
Table S2.2 (continued)

\begin{tabular}{|c|c|c|c|}
\hline 16 & $\begin{array}{l}\text { Outcomes and } \\
\text { estimations }\end{array}$ & Measures of precision & $\begin{array}{l}\text { The measure was mentioned }=\text { Fully reported } \\
=2 \\
\text { None was mentioned }=\text { No reported }=0\end{array}$ \\
\hline $17 \mathrm{a}$ & Adverse events & $\begin{array}{l}\text { Any important adverse events in } \\
\text { each group }\end{array}$ & $\begin{array}{l}\text { Any adverse event was mentioned }=\text { Fully } \\
\text { reported }=2 \\
\text { None was mentioned }=\text { No reported }=0\end{array}$ \\
\hline $17 b$ & Adverse events & $\begin{array}{l}\text { Optional item: } \\
\text { If applicable, changes of the } \\
\text { experiments were made to decrease } \\
\text { adverse events }\end{array}$ & $\begin{array}{l}\text { The improvements were mentioned }=\text { Fully } \\
\text { reported }=2 \\
\text { None was mentioned }=\text { No reported }=0 \\
\text { If not applicable, marked as '---' }\end{array}$ \\
\hline \multicolumn{4}{|c|}{ Discussion } \\
\hline $18 \mathrm{a}$ & $\begin{array}{l}\text { Interpretation/ } \\
\text { scientific } \\
\text { implications }\end{array}$ & Interpretation of results & $\begin{array}{l}\text { The interpretation was mentioned }=\text { Fully } \\
\text { reported }=2 \\
\text { None was mentioned }=\text { No reported }=0\end{array}$ \\
\hline $18 \mathrm{~b}$ & $\begin{array}{l}\text { Interpretation/ } \\
\text { scientific } \\
\text { implications }\end{array}$ & limitations of research & $\begin{array}{l}\text { The limitations were mentioned }=\text { Fully } \\
\text { reported }=2 \\
\text { None was mentioned }=\text { No reported }=0\end{array}$ \\
\hline $18 \mathrm{c}$ & $\begin{array}{l}\text { Interpretation/ } \\
\text { scientific } \\
\text { implications }\end{array}$ & Any statement of $3 \mathrm{Rs}$ & $\begin{array}{l}\text { The statements were mentioned }=\text { Fully } \\
\text { reported }=2 \\
\text { None was mentioned }=\text { No reported }=0\end{array}$ \\
\hline 19 & $\begin{array}{l}\text { Generalisability } \\
\text { translation }\end{array}$ & $\begin{array}{l}\text { Translation of the findings to other } \\
\text { species or human biology }\end{array}$ & $\begin{array}{l}\text { The translations were mentioned }=\text { Fully } \\
\text { reported }=2 \\
\text { None was mentioned }=\text { No reported }=0\end{array}$ \\
\hline 20 & Funding & $\begin{array}{l}\text { 1. Funder } \\
\text { 2. Grant number } \\
\text { 3. The role of the funders }\end{array}$ & $\begin{array}{l}\text { All the three items were mentioned }=\text { Fully } \\
\text { reported }=2 \\
\text { Declared with no conflicts }=\text { Fully reported }= \\
2 \\
\text { One of the three items was mentioned }= \\
\text { Partially reported }=1 \\
\text { None was mentioned }=\text { No reported }=0\end{array}$ \\
\hline
\end{tabular}

b

Essential item in ARRIVE-2020

\begin{tabular}{|c|c|c|c|}
\hline \multicolumn{2}{|c|}{$\begin{array}{l}\text { ARRIVE-2020 Essential } \\
10 \text { items }\end{array}$} & \multirow{2}{*}{$\begin{array}{l}\text { Evaluated by the same criteria in } \\
\text { ARRIVE- } 2010 \\
6 \mathrm{a}\end{array}$} & \multirow[t]{3}{*}{ Score } \\
\hline Study design & $1 \mathrm{a}$ & & \\
\hline & $1 b$ & $6 c$ & \\
\hline \multirow[t]{2}{*}{ Sample size } & $2 \mathrm{a}$ & $10 \mathrm{a}$ & \\
\hline & $2 b$ & $10 \mathrm{~b}$ & \\
\hline \multirow[t]{3}{*}{$\begin{array}{l}\text { Inclusion } \\
\text { exclusion criteria }\end{array}$} & and $3 a$ & A priori for Inclusion and exclusion & $\begin{array}{l}\text { The priori was mentioned }=\text { Fully } \\
\text { reported }=2\end{array}$ \\
\hline & $3 b$ & Excluded animals and reason & $\begin{array}{l}\text { None was mentioned }=\text { No reported }=0 \\
\text { The exclusion and reason were } \\
\text { mentioned }=\text { Fully reported }=2 \\
\text { None was mentioned }=\text { No reported }=0\end{array}$ \\
\hline & $3 c$ & $15 \mathrm{a}$ & \\
\hline \multirow[t]{2}{*}{ Randomisation } & $4 a$ & $11 \mathrm{a}$ & \\
\hline & $4 \mathrm{~b}$ & $11 \mathrm{~b}$ & \\
\hline
\end{tabular}


Table S2.2 (continued)

\begin{tabular}{|c|c|c|c|}
\hline Blinding & 5 & $\begin{array}{l}\text { 1. Blinding of allocation } \\
\text { 2. Blinding for performing } \\
\text { experiment } \\
\text { 3. Blinding of outcome assessment } \\
\text { 4.Blinding of data analysis }\end{array}$ & $\begin{array}{l}\text { The four items were both mentioned }= \\
\text { Fully reported }=2 \\
\text { Only one of the four items was } \\
\text { mentioned = Partially reported }=1 \\
\text { None was mentioned }=\text { No reported }=0\end{array}$ \\
\hline \multirow[t]{2}{*}{ Outcome measures } & $6 a$ & 12 & \\
\hline & $6 \mathrm{~b}$ & Not evaluated & \\
\hline \multirow[t]{3}{*}{ Statistical methods } & $7 \mathrm{a}$ & $13 \mathrm{a}$ & \\
\hline & $7 \mathrm{~b}$ & $13 b$ & \\
\hline & $7 \mathrm{c}$ & $13 \mathrm{c}$ & \\
\hline \multirow{2}{*}{$\begin{array}{l}\text { Experimental } \\
\text { animals }\end{array}$} & $8 \mathrm{a}$ & $8 \mathrm{a}$ & \\
\hline & $8 b$ & $8 \mathrm{~b}$ & \\
\hline \multirow{4}{*}{$\begin{array}{l}\text { Experimental } \\
\text { procedures }\end{array}$} & $9 \mathrm{a}$ & $7 \mathrm{a}$ & \\
\hline & $9 b$ & $7 \mathrm{~b}$ & \\
\hline & $9 \mathrm{c}$ & $7 \mathrm{c}$ & \\
\hline & $9 \mathrm{~d}$ & $7 d$ & \\
\hline \multirow[t]{2}{*}{ Results } & $10 \mathrm{a}$ & 16 & \\
\hline & $10 \mathrm{~b}$ & Not evaluated & \\
\hline
\end{tabular}

Table S2.3 Comparison of articles per (sub)item between pre-ARRIVE and post-ARRIVE for rat studies. Data are displayed as percentages of articles per score or 'yes' (or 'reported') of all the articles in that score. *represents essential (sub)items in the ARRIVE-2020 guidelines.

\begin{tabular}{|c|c|c|c|c|c|c|c|}
\hline \multirow{2}{*}{$\begin{array}{l}\text { Item } \\
1\end{array}$} & \multirow{2}{*}{$\begin{array}{c}\text { Score } \\
0,\end{array}$} & \multicolumn{2}{|c|}{$\begin{array}{l}\text { Pre-ARRIVE } \\
\mathrm{n}=55 \text { per item }\end{array}$} & \multicolumn{2}{|c|}{$\begin{array}{l}\text { Post-ARRIVE } \\
\mathrm{n}=88 \text { per item }\end{array}$} & \multirow{2}{*}{$\begin{array}{c}P \text {-value } \\
0.207\end{array}$} & \multirow[t]{2}{*}{ Description } \\
\hline & & 31 & $56 \%$ & 43 & $49 \%$ & & \\
\hline & 1 & 0 & $0 \%$ & 5 & $6 \%$ & & \\
\hline & 2 , & 24 & $44 \%$ & 40 & $45 \%$ & & \\
\hline \multirow[t]{3}{*}{2} & 0 , & 0 & $0 \%$ & 0 & $0 \%$ & 0.722 & \\
\hline & 1 , & 36 & $65 \%$ & 54 & $61 \%$ & & \\
\hline & 2 & 19 & $35 \%$ & 34 & $39 \%$ & & \\
\hline \multirow[t]{2}{*}{$3 \mathrm{a}$} & No, & 0 & $0 \%$ & 0 & $0 \%$ & & All articles with 'fully reported' for \\
\hline & Yes, & 55 & $100 \%$ & 88 & $100 \%$ & & pre-ARRIVE \\
\hline \multirow[t]{3}{*}{$3 b$} & 0 & 35 & $64 \%$ & 55 & $63 \%$ & 0.625 & \\
\hline & 1 , & 12 & $22 \%$ & 24 & $27 \%$ & & \\
\hline & 2 & 8 & $15 \%$ & 9 & $10 \%$ & & \\
\hline \multirow[t]{2}{*}{4} & No, & 0 & $0 \%$ & 1 & $1 \%$ & 1.000 & All articles with 'fully reported' for \\
\hline & Yes, & 55 & $100 \%$ & 87 & $99 \%$ & & pre-ARRIVE \\
\hline \multirow[t]{3}{*}{5} & 0 & 0 & $0 \%$ & 5 & $6 \%$ & 0.216 & \\
\hline & 1 , & 23 & $42 \%$ & 38 & $43 \%$ & & \\
\hline & 2 & 32 & $58 \%$ & 45 & $51 \%$ & & \\
\hline \multirow[t]{2}{*}{$6 a^{*}$} & No, & 1 & $2 \%$ & 0 & $0 \%$ & 0.380 & $>90 \%$ articles with 'fully reported' \\
\hline & Yes, & 54 & $98 \%$ & 88 & $100 \%$ & & for pre-ARRIVE \\
\hline \multirow[t]{3}{*}{$6 b$} & 0 & 17 & $31 \%$ & 40 & $45 \%$ & 0.145 & \\
\hline & 1, & 36 & $65 \%$ & 47 & $53 \%$ & & \\
\hline & 2 & 2 & $4 \%$ & 1 & $1 \%$ & & \\
\hline \multirow[t]{2}{*}{$6 c^{*}$} & No, & 52 & $95 \%$ & 82 & $93 \%$ & 1.000 & \\
\hline & Yes, & 3 & $5 \%$ & 6 & $7 \%$ & & \\
\hline
\end{tabular}


Table S2.3 (continued)

\begin{tabular}{|c|c|c|c|c|c|c|c|}
\hline \multirow[t]{4}{*}{$7 a^{*}$} & 0 & 1 & $2 \%$ & 1 & $1 \%$ & & \multirow{4}{*}{$\begin{array}{l}\text { Score } 2 \text { presents the most significant } \\
\text { difference }\end{array}$} \\
\hline & 1, & 5 & $9 \%$ & 19 & $22 \%$ & 0.004 & \\
\hline & 2 & 40 & $73 \%$ & 39 & $44 \%$ & & \\
\hline & 3 & 9 & $16 \%$ & 29 & $33 \%$ & & \\
\hline \multirow[t]{2}{*}{$7 b^{*}$} & No, & 55 & $100 \%$ & 88 & $100 \%$ & & \multirow[t]{5}{*}{ None reported } \\
\hline & Yes, & 0 & $0 \%$ & 0 & $0 \%$ & & \\
\hline \multirow[t]{3}{*}{$7 \mathrm{c}^{*}$} & 0 & 36 & $65 \%$ & 66 & $75 \%$ & 0.409 & \\
\hline & 1, & 18 & $33 \%$ & 20 & $23 \%$ & & \\
\hline & 2 & 1 & $2 \%$ & 2 & $2 \%$ & & \\
\hline \multirow[t]{3}{*}{$7 d^{*}$} & 0 , & 54 & $98 \%$ & 88 & $100 \%$ & 0.385 & \\
\hline & 1, & 1 & $2 \%$ & 0 & $0 \%$ & & \\
\hline & 2 & 0 & $0 \%$ & 0 & $0 \%$ & & \\
\hline \multirow[t]{4}{*}{$8 a^{*}$} & 0 , & 0 & $0 \%$ & 0 & $0 \%$ & & \\
\hline & 1, & 1 & $2 \%$ & 4 & $5 \%$ & 0.230 & \\
\hline & 2 & 41 & $75 \%$ & 53 & $60 \%$ & & \\
\hline & 3 , & 13 & $24 \%$ & 31 & $35 \%$ & & \\
\hline \multirow[t]{3}{*}{$8 b^{*}$} & 0 , & 26 & $47 \%$ & 37 & $42 \%$ & 0.883 & \\
\hline & 1, & 26 & $47 \%$ & 46 & $52 \%$ & & \\
\hline & 2 & 3 & $5 \%$ & 5 & $6 \%$ & & \\
\hline \multirow[t]{3}{*}{$9 \mathrm{a}$} & 0 , & 32 & $58 \%$ & 56 & $64 \%$ & 0.670 & \\
\hline & 1, & 21 & $38 \%$ & 30 & $34 \%$ & & \\
\hline & 2 & 2 & $4 \%$ & 2 & $2 \%$ & & \\
\hline \multirow[t]{4}{*}{$9 b$} & 0 , & 21 & $38 \%$ & 37 & $42 \%$ & & \\
\hline & 1, & 14 & $25 \%$ & 29 & $33 \%$ & 0.102 & \\
\hline & 2 & 20 & $36 \%$ & 18 & $20 \%$ & & \\
\hline & 3 , & 0 & $0 \%$ & 4 & $5 \%$ & & \\
\hline \multirow[t]{3}{*}{$9 c$} & 0 , & 27 & $49 \%$ & 35 & $40 \%$ & 0.042 & \multirow{6}{*}{$\begin{array}{l}\text { Score } 2 \text { presents the most significant } \\
\text { difference }\end{array}$} \\
\hline & 1, & 22 & $40 \%$ & 28 & $32 \%$ & & \\
\hline & 2, & 6 & $11 \%$ & 25 & $28 \%$ & & \\
\hline \multirow[t]{3}{*}{$10 a^{*}$} & 0 , & 0 & $0 \%$ & 3 & $3 \%$ & 0.149 & \\
\hline & 1, & 8 & $15 \%$ & 6 & $7 \%$ & & \\
\hline & 2 & 47 & $85 \%$ & 79 & $90 \%$ & & \\
\hline \multirow[t]{2}{*}{$10 b^{*}$} & No, & 51 & $93 \%$ & 83 & $94 \%$ & 0.978 & \\
\hline & Yes, & 4 & $7 \%$ & 5 & $6 \%$ & & \\
\hline \multirow[t]{2}{*}{$11 a^{*}$} & No, & 52 & $95 \%$ & 83 & $94 \%$ & 1.000 & \\
\hline & Yes, & 3 & $5 \%$ & 5 & $6 \%$ & & \\
\hline \multirow[t]{2}{*}{$11 b^{*}$} & No, & 51 & $93 \%$ & 85 & $97 \%$ & 0.520 & \\
\hline & Yes, & 4 & $7 \%$ & 3 & $3 \%$ & & \\
\hline \multirow[t]{2}{*}{$12 *$} & No, & 0 & $0 \%$ & 0 & $0 \%$ & & All articles with 'fully reported' for \\
\hline & Yes, & 55 & $100 \%$ & 88 & $100 \%$ & & pre-ARRIVE \\
\hline \multirow[t]{2}{*}{$13 a^{*}$} & No, & 3 & $5 \%$ & 4 & $5 \%$ & 1.000 & $>90 \%$ articles with 'fully reported' \\
\hline & Yes, & 52 & $95 \%$ & 84 & $95 \%$ & & for pre-ARRIVE \\
\hline \multirow[t]{2}{*}{$13 b^{*}$} & No, & 54 & $98 \%$ & 87 & $99 \%$ & 1.000 & \\
\hline & Yes, & 1 & $2 \%$ & 1 & $1 \%$ & & \\
\hline \multirow[t]{2}{*}{$13 c^{*}$} & No, & 43 & $78 \%$ & 57 & $65 \%$ & 0.089 & \\
\hline & Yes, & 12 & $22 \%$ & 31 & $35 \%$ & & \\
\hline 14 & 0 & 48 & $87 \%$ & 67 & $76 \%$ & 0.076 & \\
\hline & 1 , & 7 & $13 \%$ & 21 & $24 \%$ & & \\
\hline & 2 & 0 & $0 \%$ & 0 & $0 \%$ & & \\
\hline $15 a^{*}$ & No, & 21 & $38 \%$ & 37 & $42 \%$ & 0.647 & \\
\hline & Yes, & 34 & $62 \%$ & 51 & $58 \%$ & & \\
\hline
\end{tabular}


Table S2.3 (continued)

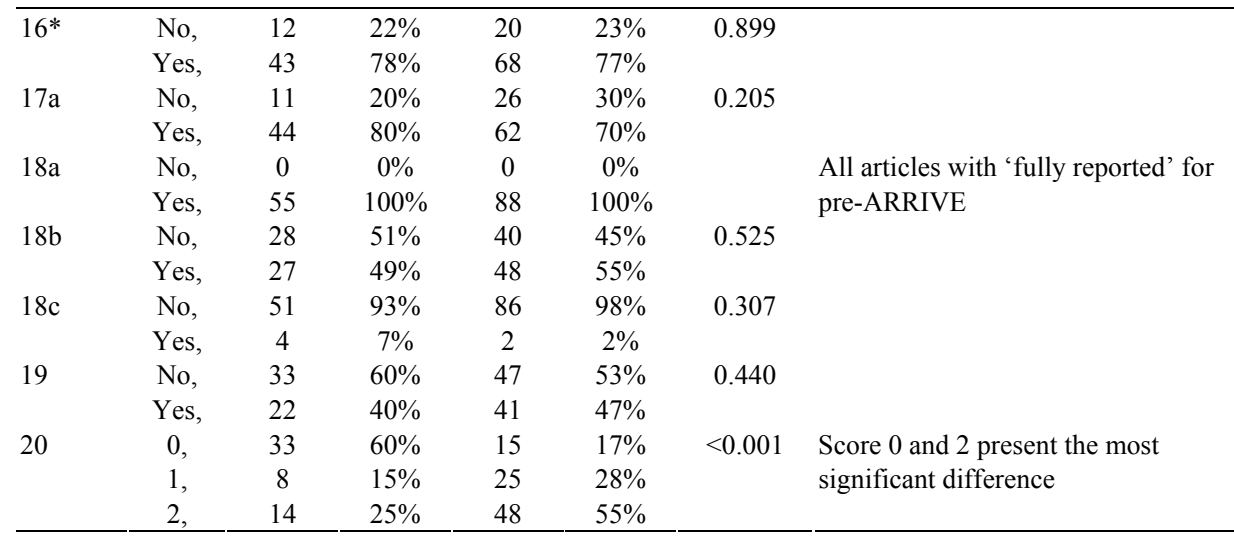

Table S2.4 Comparison of articles per (sub)item between pre-ARRIVE and post-ARRIVE for rabbit studies. Data are displayed as percentages of articles per score or 'yes' (or 'reported') of all the articles in that score. *represents essential (sub)items in the ARRIVE-2020 guidelines.

\begin{tabular}{|c|c|c|c|c|c|c|c|}
\hline \multirow{2}{*}{$\begin{array}{l}\text { Item } \\
1\end{array}$} & \multirow{2}{*}{$\begin{array}{c}\text { Score } \\
0,\end{array}$} & \multicolumn{2}{|c|}{$\begin{array}{c}\text { Pre } \\
\mathrm{n}=29 \text { per item }\end{array}$} & \multicolumn{2}{|c|}{$\begin{array}{c}\text { Post } \\
n=37 \text { per item }\end{array}$} & \multirow{4}{*}{$\begin{array}{c}P \text {-value } \\
0.037\end{array}$} & \multirow{3}{*}{$\begin{array}{l}\text { Description } \\
\text { Score2 presents the most significant } \\
\text { difference }\end{array}$} \\
\hline & & 15 & $52 \%$ & 26 & $70 \%$ & & \\
\hline & 1, & 0 & $0 \%$ & 3 & $8 \%$ & & \\
\hline & 2 , & 14 & $48 \%$ & 8 & $22 \%$ & & \\
\hline \multirow[t]{3}{*}{2} & 0 & 0 & $0 \%$ & 0 & $0 \%$ & 0.620 & \\
\hline & 1, & 14 & $48 \%$ & 21 & $57 \%$ & & \\
\hline & 2 & 15 & $52 \%$ & 16 & $43 \%$ & & \\
\hline \multirow[t]{2}{*}{$3 a$} & No, & 0 & $0 \%$ & 0 & $0 \%$ & 0.620 & All articles with 'fully reported' for \\
\hline & Yes, & 29 & $100 \%$ & 37 & $100 \%$ & & pre-ARRIVE \\
\hline \multirow[t]{3}{*}{$3 b$} & 0 & 20 & $69 \%$ & 26 & $70 \%$ & 1.000 & \\
\hline & 1 , & 6 & $21 \%$ & 7 & $19 \%$ & & \\
\hline & 2 & 3 & $10 \%$ & 4 & $11 \%$ & & \\
\hline \multirow[t]{2}{*}{4} & No, & 0 & $0 \%$ & 0 & $0 \%$ & & All articles with 'fully reported' for \\
\hline & Yes, & 29 & $100 \%$ & 37 & $100 \%$ & & pre-ARRIVE \\
\hline \multirow[t]{3}{*}{5} & 0 & 1 & $3 \%$ & 0 & $0 \%$ & $<0.001$ & Score 1 and 2 present the most \\
\hline & 1 , & 17 & $59 \%$ & 4 & $11 \%$ & & significant difference \\
\hline & 2 & 11 & $38 \%$ & 33 & $89 \%$ & & \\
\hline \multirow[t]{2}{*}{$6 a^{*}$} & No, & 1 & $3 \%$ & 0 & $0 \%$ & 0.439 & $>90 \%$ articles with 'fully reported' \\
\hline & Yes, & 28 & $97 \%$ & 37 & $100 \%$ & & for pre-ARRIVE \\
\hline \multirow[t]{3}{*}{$6 b$} & 0 & 15 & $52 \%$ & 11 & $30 \%$ & 0.194 & \\
\hline & 1 , & 12 & $41 \%$ & 22 & $59 \%$ & & \\
\hline & 2 & 2 & $7 \%$ & 4 & $11 \%$ & & \\
\hline \multirow[t]{2}{*}{$6 c^{*}$} & No, & 25 & $86 \%$ & 35 & $95 \%$ & 0.456 & \\
\hline & Yes, & 4 & $14 \%$ & 2 & $5 \%$ & & \\
\hline \multirow[t]{4}{*}{$7 a^{*}$} & 0 & 0 & $0 \%$ & 0 & $0 \%$ & 0.023 & Score 2 and 3 present the most \\
\hline & 1 , & 1 & $3 \%$ & 1 & $3 \%$ & & significant difference \\
\hline & 2 & 22 & $76 \%$ & 17 & $46 \%$ & & \\
\hline & 3 & 6 & $21 \%$ & 19 & $51 \%$ & & \\
\hline \multirow[t]{2}{*}{$7 b^{*}$} & No, & 29 & $100 \%$ & 37 & $100 \%$ & & None reported \\
\hline & Yes, & 0 & $0 \%$ & 0 & $0 \%$ & & \\
\hline
\end{tabular}


Table S2.4 (continued)

\begin{tabular}{|c|c|c|c|c|c|c|c|}
\hline \multirow[t]{3}{*}{$7 c^{*}$} & 0 & 19 & $66 \%$ & 29 & $78 \%$ & 0.475 & \\
\hline & 1 & 8 & $28 \%$ & 7 & $19 \%$ & & \\
\hline & 2 & 2 & $7 \%$ & 1 & $3 \%$ & & \\
\hline \multirow[t]{3}{*}{$7 d^{*}$} & 0 & 29 & $100 \%$ & 37 & $100 \%$ & & \\
\hline & 1, & 0 & $0 \%$ & 0 & $0 \%$ & & None reported \\
\hline & 2 & 0 & $0 \%$ & 0 & $0 \%$ & & \\
\hline \multirow{4}{*}{$8 a^{*}$} & 0 & 0 & $0 \%$ & 0 & $0 \%$ & 0.852 & \\
\hline & 1, & 4 & $14 \%$ & 3 & $8 \%$ & & \\
\hline & 2 & 20 & $69 \%$ & 27 & $73 \%$ & & \\
\hline & 3 , & 5 & $17 \%$ & 7 & $19 \%$ & & \\
\hline \multirow[t]{3}{*}{$8 b^{*}$} & 0 & 24 & $83 \%$ & 26 & $70 \%$ & 0.189 & \\
\hline & 1 , & 5 & $17 \%$ & 11 & $30 \%$ & & \\
\hline & 2 & 0 & $0 \%$ & 0 & $0 \%$ & & \\
\hline \multirow[t]{3}{*}{$9 a$} & 0 & 24 & $83 \%$ & 28 & $76 \%$ & 0.349 & \\
\hline & 1, & 5 & $17 \%$ & 9 & $24 \%$ & & \\
\hline & 2 & 0 & $0 \%$ & 0 & $0 \%$ & & \\
\hline \multirow[t]{4}{*}{$9 b$} & 0 & 9 & $31 \%$ & 21 & $57 \%$ & 0.094 & \\
\hline & 1, & 13 & $45 \%$ & 9 & $24 \%$ & & \\
\hline & 2 & 6 & $21 \%$ & 7 & $19 \%$ & & \\
\hline & 3 & 1 & $3 \%$ & 0 & $0 \%$ & & \\
\hline \multirow[t]{3}{*}{$9 \mathrm{c}$} & 0 & 17 & $59 \%$ & 11 & $30 \%$ & 0.005 & Score 2 presents the most \\
\hline & 1 , & 9 & $31 \%$ & 9 & $24 \%$ & & significant difference \\
\hline & 2 , & 3 & $10 \%$ & 17 & $46 \%$ & & \\
\hline \multirow[t]{3}{*}{$10 a^{*}$} & 0 , & 1 & $3 \%$ & 0 & $0 \%$ & 0.803 & $>90 \%$ articles with 'fully reported' \\
\hline & 1 , & 2 & $7 \%$ & 3 & $8 \%$ & & for pre-ARRIVE \\
\hline & 2 & 26 & $90 \%$ & 34 & $92 \%$ & & \\
\hline \multirow[t]{2}{*}{$10 b^{*}$} & No, & 27 & $93 \%$ & 36 & $97 \%$ & 0.829 & \\
\hline & Yes, & 2 & $7 \%$ & 1 & $3 \%$ & & \\
\hline \multirow[t]{2}{*}{$11 a^{*}$} & No, & 24 & $83 \%$ & 34 & $92 \%$ & 0.454 & \\
\hline & Yes, & 5 & $17 \%$ & 3 & $8 \%$ & & \\
\hline \multirow[t]{2}{*}{$11 b^{*}$} & No, & 22 & $76 \%$ & 34 & $92 \%$ & 0.145 & \\
\hline & Yes, & 7 & $24 \%$ & 3 & $8 \%$ & & \\
\hline \multirow[t]{2}{*}{$12 *$} & No, & 0 & $0 \%$ & 0 & $0 \%$ & & All articles with 'fully reported' for \\
\hline & Yes, & 29 & $100 \%$ & 37 & $100 \%$ & & pre-ARRIVE \\
\hline \multirow[t]{2}{*}{$13 a^{*}$} & No, & 3 & $10 \%$ & 2 & $5 \%$ & 0.776 & $>90 \%$ articles with 'fully reported' \\
\hline & Yes, & 26 & $90 \%$ & 35 & $95 \%$ & & for pre-ARRIVE \\
\hline \multirow[t]{2}{*}{$13 b^{*}$} & No, & 29 & $100 \%$ & 35 & $95 \%$ & 0.500 & \\
\hline & Yes, & 0 & $0 \%$ & 2 & $5 \%$ & & \\
\hline \multirow[t]{2}{*}{$13 \mathrm{c} *$} & No, & 26 & $90 \%$ & 25 & $68 \%$ & 0.034 & \\
\hline & Yes, & 3 & $10 \%$ & 12 & $32 \%$ & & \\
\hline \multirow[t]{3}{*}{14} & 0 & 26 & $90 \%$ & 28 & $76 \%$ & 0.126 & \\
\hline & 1, & 3 & $10 \%$ & 9 & $24 \%$ & & \\
\hline & 2 & 0 & $0 \%$ & 0 & $0 \%$ & & \\
\hline \multirow[t]{2}{*}{$15 a^{*}$} & No, & 14 & $48 \%$ & 17 & $46 \%$ & 0.851 & \\
\hline & Yes, & 15 & $52 \%$ & 20 & $54 \%$ & & \\
\hline \multirow[t]{2}{*}{$16^{*}$} & No, & 9 & $31 \%$ & 5 & $14 \%$ & 0.084 & \\
\hline & Yes, & 20 & $69 \%$ & 32 & $86 \%$ & & \\
\hline \multirow[t]{2}{*}{$17 \mathrm{a}$} & No, & 6 & $21 \%$ & 4 & $11 \%$ & 0.444 & \\
\hline & Yes, & 23 & $79 \%$ & 33 & $89 \%$ & & \\
\hline \multirow[t]{2}{*}{$18 \mathrm{a}$} & No, & 0 & $0 \%$ & 0 & $0 \%$ & & All articles with 'fully reported' for \\
\hline & Yes, & 29 & $100 \%$ & 37 & $100 \%$ & & pre-ARRIVE \\
\hline
\end{tabular}


Table S2.4 (continued)

\begin{tabular}{cccccccl}
\hline $18 \mathrm{~b}$ & No, & 19 & $66 \%$ & 9 & $24 \%$ & 0.001 & \\
& Yes, & 10 & $34 \%$ & 28 & $76 \%$ & & \\
$18 \mathrm{c}$ & No, & 29 & $100 \%$ & 30 & $81 \%$ & 0.038 & \\
& Yes, & 0 & $0 \%$ & 7 & $19 \%$ & & \\
19 & No, & 17 & $59 \%$ & 16 & $43 \%$ & 0.215 & \\
& Yes, & 12 & $41 \%$ & 21 & $57 \%$ & & \\
& 0, & 11 & $38 \%$ & 0 & $0 \%$ & $<0.001$ & Score 0 and 2 present the most \\
& 1, & 13 & $45 \%$ & 15 & $41 \%$ & & significant difference \\
& 2, & 5 & $17 \%$ & 22 & $59 \%$ & & \\
\hline
\end{tabular}

Table S2.5 Comparison of articles per (sub)item between pre-ARRIVE and post-ARRIVE for pig studies. Data are displayed as percentages of articles per score or 'yes' (or 'reported') of all the articles in that score. *represents essential (sub)items in the ARRIVE-2020 guidelines.

\begin{tabular}{|c|c|c|c|c|c|c|c|}
\hline \multirow{2}{*}{$\begin{array}{l}\text { Item } \\
1\end{array}$} & \multirow{2}{*}{$\begin{array}{c}\text { Score } \\
0,\end{array}$} & \multicolumn{2}{|c|}{$\begin{array}{l}\text { Pre-ARRIVE } \\
\mathrm{n}=17 \text { per item }\end{array}$} & \multicolumn{2}{|c|}{$\begin{array}{l}\text { Post-ARRIVE } \\
n=18 \text { per item }\end{array}$} & \multirow{3}{*}{$\begin{array}{c}\text { P-value } \\
0.004\end{array}$} & \multirow{3}{*}{$\begin{array}{l}\text { Description } \\
\text { Score } 0 \text { and } 2 \text { present the most } \\
\text { significant difference }\end{array}$} \\
\hline & & 9 & $53 \%$ & 1 & $6 \%$ & & \\
\hline & 1 & 0 & $0 \%$ & 1 & $6 \%$ & & \\
\hline & 2 & 8 & $47 \%$ & 16 & $89 \%$ & & \\
\hline \multirow[t]{3}{*}{2} & 0 & 0 & $0 \%$ & 0 & $0 \%$ & 0.773 & \\
\hline & 1 , & 11 & $65 \%$ & 10 & $56 \%$ & & \\
\hline & 2 & 6 & $35 \%$ & 8 & $44 \%$ & & \\
\hline \multirow[t]{2}{*}{$3 \mathrm{a}$} & No, & 0 & $0 \%$ & 0 & $0 \%$ & & All articles with 'fully reported' for \\
\hline & Yes, & 17 & $100 \%$ & 18 & $100 \%$ & & pre-ARRIVE \\
\hline \multirow[t]{3}{*}{$3 b$} & 0 & 8 & $47 \%$ & 10 & $56 \%$ & 0.401 & \\
\hline & 1 , & 8 & $47 \%$ & 5 & $28 \%$ & & \\
\hline & 2 & 1 & $6 \%$ & 3 & $17 \%$ & & \\
\hline \multirow[t]{2}{*}{4} & No, & 0 & $0 \%$ & 0 & $0 \%$ & & All articles with 'fully reported' for \\
\hline & Yes, & 17 & $100 \%$ & 18 & $100 \%$ & & pre-ARRIVE \\
\hline \multirow[t]{3}{*}{5} & 0 & 1 & $6 \%$ & 2 & $11 \%$ & 0.399 & \\
\hline & 1 , & 11 & $65 \%$ & 7 & $39 \%$ & & \\
\hline & 2 & 5 & $29 \%$ & 9 & $50 \%$ & & \\
\hline \multirow[t]{2}{*}{$6 a^{*}$} & No, & 4 & $24 \%$ & 0 & $0 \%$ & 0.045 & \\
\hline & Yes, & 13 & $76 \%$ & 18 & $100 \%$ & & \\
\hline \multirow[t]{3}{*}{$6 b$} & 0 & 9 & $53 \%$ & 4 & $22 \%$ & 0.118 & \\
\hline & 1, & 8 & $47 \%$ & 13 & $72 \%$ & & \\
\hline & 2 & 0 & $0 \%$ & 1 & $6 \%$ & & \\
\hline \multirow[t]{2}{*}{$6 c^{*}$} & No, & 17 & $100 \%$ & 15 & $83 \%$ & 0.229 & \\
\hline & Yes, & 0 & $0 \%$ & 3 & $17 \%$ & & \\
\hline \multirow[t]{4}{*}{$7 a^{*}$} & 0 & 0 & $0 \%$ & 0 & $0 \%$ & 0.324 & \\
\hline & 1 , & 5 & $29 \%$ & 3 & $17 \%$ & & \\
\hline & 2 & 7 & $41 \%$ & 5 & $28 \%$ & & \\
\hline & 3 & 5 & $29 \%$ & 10 & $56 \%$ & & \\
\hline \multirow[t]{2}{*}{$7 b^{*}$} & No, & 17 & $100 \%$ & 18 & $100 \%$ & & None reported \\
\hline & Yes, & 0 & $0 \%$ & 0 & $0 \%$ & & \\
\hline \multirow[t]{3}{*}{$7 c^{*}$} & 0 & 13 & $76 \%$ & 10 & $56 \%$ & 0.378 & \\
\hline & 1 , & 3 & $18 \%$ & 4 & $22 \%$ & & \\
\hline & 2 & 1 & $6 \%$ & 4 & $22 \%$ & & \\
\hline
\end{tabular}


Table S2.5 (continued)

\begin{tabular}{|c|c|c|c|c|c|c|c|}
\hline \multirow[t]{3}{*}{$7 d^{*}$} & 0 & 16 & $94 \%$ & 18 & $100 \%$ & 0.486 & \\
\hline & 1, & 1 & $6 \%$ & 0 & $0 \%$ & & \\
\hline & 2 & 0 & $0 \%$ & 0 & $0 \%$ & & \\
\hline \multirow[t]{4}{*}{$8 a^{*}$} & 0 & 0 & $0 \%$ & 0 & $0 \%$ & 0.196 & \\
\hline & 1, & 2 & $12 \%$ & 2 & $11 \%$ & & \\
\hline & 2 & 15 & $88 \%$ & 12 & $67 \%$ & & \\
\hline & 3 , & 0 & $0 \%$ & 4 & $22 \%$ & & \\
\hline \multirow[t]{3}{*}{$8 b^{*}$} & 0 & 14 & $82 \%$ & 10 & $56 \%$ & 0.150 & \\
\hline & 1, & 3 & $18 \%$ & 5 & $28 \%$ & & \\
\hline & 2 , & 0 & $0 \%$ & 3 & $17 \%$ & & \\
\hline \multirow[t]{3}{*}{$9 a$} & 0 & 16 & $94 \%$ & 17 & $94 \%$ & 1.000 & \\
\hline & 1, & 1 & $6 \%$ & 1 & $6 \%$ & & \\
\hline & 2 & 0 & $0 \%$ & 0 & $0 \%$ & & \\
\hline \multirow[t]{4}{*}{$9 b$} & 0 & 12 & $71 \%$ & 9 & $50 \%$ & 0.401 & \\
\hline & 1, & 4 & $24 \%$ & 5 & $28 \%$ & & \\
\hline & 2 & 1 & $6 \%$ & 4 & $22 \%$ & & \\
\hline & 3 , & 0 & $0 \%$ & 0 & $0 \%$ & & \\
\hline \multirow[t]{3}{*}{$9 c$} & 0 , & 9 & $53 \%$ & 7 & $39 \%$ & 0.293 & \\
\hline & 1, & 7 & $41 \%$ & 6 & $33 \%$ & & \\
\hline & 2 & 1 & $6 \%$ & 5 & $28 \%$ & & \\
\hline \multirow[t]{3}{*}{$10 \mathrm{a}^{*}$} & 0 & 1 & $6 \%$ & 0 & $0 \%$ & 0.823 & \\
\hline & 1, & 3 & $18 \%$ & 3 & $17 \%$ & & \\
\hline & 2 & 13 & $76 \%$ & 15 & $83 \%$ & & \\
\hline \multirow[t]{2}{*}{$10 b^{*}$} & No, & 16 & $94 \%$ & 18 & $100 \%$ & 0.486 & \\
\hline & Yes, & 1 & $6 \%$ & 0 & $0 \%$ & & \\
\hline \multirow[t]{2}{*}{$11 \mathrm{a} *$} & No, & 15 & $88 \%$ & 18 & $100 \%$ & 0.229 & \\
\hline & Yes, & 2 & $12 \%$ & 0 & $0 \%$ & & \\
\hline \multirow[t]{2}{*}{$11 b^{*}$} & No, & 11 & $65 \%$ & 16 & $89 \%$ & 0.121 & \\
\hline & Yes, & 6 & $35 \%$ & 2 & $11 \%$ & & \\
\hline \multirow[t]{2}{*}{$12 *$} & No, & 0 & $0 \%$ & 0 & $0 \%$ & & All articles with 'fully reported' for \\
\hline & Yes, & 17 & $100 \%$ & 18 & $100 \%$ & & pre-ARRIVE \\
\hline \multirow[t]{2}{*}{$13 \mathrm{a} *$} & No, & 3 & $18 \%$ & 0 & $0 \%$ & 0.104 & \\
\hline & Yes, & 14 & $82 \%$ & 18 & $100 \%$ & & \\
\hline \multirow[t]{2}{*}{$13 b^{*}$} & No, & 17 & $100 \%$ & 18 & $100 \%$ & & None reported \\
\hline & Yes, & 0 & $0 \%$ & 0 & $0 \%$ & & \\
\hline \multirow[t]{2}{*}{$13 c^{*}$} & No, & 12 & $71 \%$ & 12 & $67 \%$ & 1.000 & \\
\hline & Yes, & 5 & $29 \%$ & 6 & $33 \%$ & & \\
\hline \multirow[t]{3}{*}{14} & 0 & 12 & $71 \%$ & 13 & $72 \%$ & 1.000 & \\
\hline & 1 , & 5 & $29 \%$ & 5 & $28 \%$ & & \\
\hline & 2 & 0 & $0 \%$ & 0 & $0 \%$ & & \\
\hline \multirow[t]{2}{*}{$15 \mathrm{a}^{*}$} & No, & 8 & $47 \%$ & 8 & $44 \%$ & 1.000 & \\
\hline & Yes, & 9 & $53 \%$ & 10 & $56 \%$ & & \\
\hline \multirow[t]{2}{*}{$16^{*}$} & No, & 9 & $53 \%$ & 7 & $39 \%$ & 0.505 & \\
\hline & Yes, & 8 & $47 \%$ & 11 & $61 \%$ & & \\
\hline \multirow[t]{2}{*}{$17 \mathrm{a}$} & No, & 6 & $35 \%$ & 7 & $39 \%$ & 1.000 & \\
\hline & Yes, & 11 & $65 \%$ & 11 & $61 \%$ & & \\
\hline \multirow[t]{2}{*}{$18 \mathrm{a}$} & No, & 0 & $0 \%$ & 0 & $0 \%$ & & All articles with 'fully reported' for \\
\hline & Yes, & 17 & $100 \%$ & 18 & $100 \%$ & & pre-ARRIVE \\
\hline \multirow[t]{2}{*}{$18 \mathrm{~b}$} & No, & 6 & $35 \%$ & 3 & $17 \%$ & 0.264 & \\
\hline & Yes, & 11 & $65 \%$ & 15 & $83 \%$ & & \\
\hline
\end{tabular}


Chapter 2

Table S2.5 (continued)

\begin{tabular}{lcccccll}
\hline $18 \mathrm{c}$ & No, & 15 & $88 \%$ & 17 & $94 \%$ & 0.603 & \\
& Yes, & 2 & $12 \%$ & 1 & $6 \%$ & & \\
19 & No, & 9 & $53 \%$ & 5 & $28 \%$ & 0.176 & \\
& Yes, & 8 & $47 \%$ & 13 & $72 \%$ & & \\
& 0, & 10 & $59 \%$ & 0 & $0 \%$ & $<0.001$ & Score 0 and 1 present the most \\
& 1, & 7 & $41 \%$ & 15 & $83 \%$ & & significant difference \\
& 2, & 0 & $0 \%$ & 3 & $17 \%$ & & \\
\hline
\end{tabular}




\section{Chapter 3}

Baseline performance of the ischaemic button model for induction of adhesions in laboratory rats

Elwin HH Mommers, Hong Liu, Audrey Jongen, and Nicole D Bouvy Laboratory Animals 2019;53(1):63-71 


\section{Abstract}

\section{Background and objective}

The ischaemic button model is frequently used for the induction of adhesions in laboratory rats. Male rats are often used because of the common belief that the peritesticular (intra-abdominal) fat in males facilitates adhesion formation, although this theory is not evidence based. Comparing the model's performance in both sexes is an important aspect of refining animal experiments. The aim of this study is to compare baseline performance of the modified ischaemic button model in both male and female rats.

\section{Methods}

Follow-up was 1 week and noted endpoints were intra-abdominal adhesion formation and differences in welfare assessment.

\section{Results}

A total of 192 ischaemic buttons (96 male/96 female) were created in 24 Wistar Han rats (12 male/12 female). After 1 week of follow-up, 93 buttons survived in the male group $(96.9 \%)$ compared with 91 in the female group $(94.8 \%)(P=0.409)$. In the male group, 85 out of $93(91.4 \%)$ buttons induced adhesions compared with 84 out of 91 $(92.3 \%)$ in the female group $(P=0.881)$. All but one animal had a Zühlke score of 3 . There were no clinically relevant differences in welfare scores. Male animals increased in weight significantly faster compared to females $(P<0.001)$, after correcting for physiological growth.

\section{Conclusion}

The ischaemic button model resulted in equal quality and quantity of intra-abdominal adhesions in both male and female Wistar Han rats. Both male and female Wistar Han rats are suitable for the induction of experimental adhesions in the ischaemic button model. 


\section{Introduction}

Intra-abdominal postoperative adhesions are fibrotic connections between intraabdominal organs and structures that are normally unconnected. ${ }^{1}$ The adhesions are the results of a pathological healing following inflammation of the peritoneum and occur after nearly $100 \%$ of all intra-abdominal surgical procedures. ${ }^{2,3}$ Approximately $5-18 \%$ of the adhesions will become clinically relevant and cause intestinal obstruction, infertility or chronic abdominal pain. ${ }^{1,45}$ Because of the vast number of intra-abdominal procedures performed every year, intra-abdominal adhesions pose a billion dollar burden on the global health care system. ${ }^{1,3-7}$ As a consequence, the development and evaluation of antiadhesive components is a large industry that depends heavily on the use of experimental animal models that provide a consistent induction of experimental adhesions. The ischaemic button model is one of the most frequently used animal models for experimental adhesions. In short, the technique uses a suture tied around a $5 \mathrm{~mm}$ portion of the parietal peritoneum. Subsequently, the ligated tissue becomes ischaemic, resulting in the development of an adhesion. The original model described by Buckenmaier et al. is susceptible to failure of the buttons, due to slipping of the sutures. $^{8}$ In an attempt to improve the original technique, Rajab et al. introduced a modified ischaemic button model in 2010. ${ }^{9}$ Rajab et al. improved the model by introducing a backstitch ligature to reduce the number of slipped sutures and create durable ischaemic buttons. In their validation article they com-pared the modified ischaemic button model to the original model and found a lower percentage of button obliteration in the modified ischaemic button model $(18.8 \%$ vs. $3.8 \%){ }^{9}$. Since the introduction of the modified technique, it has been applied multiple times in both male and female rats in several studies. The majority of studies concern male rats, possibly because they potentially have larger fat depositions in the lower body, their epidydimal or peritesticular fat. Despite the frequent use of the ischaemic button model in animal experiments, a comparison in baseline performance between male and female rats has never been performed. Comparing baseline performance between both sexes is an important aspect of refining animal experiments and provides evidence-based data regarding the number and sex of the animals included in future research. The aim of this study is to compare baseline performance of the modified ischaemic button model in both male and female Wistar Han rats.

\section{Methods}

Before commencement of this study, ethical approval was obtained from the Dutch Central Committee of Animal Experiments and from the institutional review board of the Maastricht University Medical Centre, Animal Ethical Committee and Inspection 
for Animal Welfare (licence no. AVD107002016720). This study was performed according to the European Convention for the Protection of Vertebrate Animals used for Experimental and other Scientific Purposes (European Treaty Series (ETS) no. 123).

Twenty-four adult Wistar Han rats (12 males/12 females) aged 8 weeks (weight range: male 200-250 g, female 150-175 g) and bred by Charles River Laboratories (Sulzfeld, Germany) were socially housed in an EU type IV Macrolon cage of $1800 \mathrm{~cm}^{2}$, with cage enrichment in the form of a cardboard cylinder and a wooden block at the centralised animal experiments facility of the Maastricht University Medical Centre (Maastricht, The Netherlands). Rooms were fitted with an artificial 12-hour light/dark cycle, room temperature was standardised at $25^{\circ} \mathrm{C}$ and food and water were provided ad libitum in the form of sterilised compressed food pellets $(10 \mathrm{~mm} \mathrm{Sni}$ rat/mouse maintenance food) and acidified drinking water. All animals were followed for 7 days before being euthanised under general anaesthesia via cardiac puncture.

\section{Procedure}

All operations were performed by two surgeons (EHHM and LH) certified for working with laboratory animals. All animals were randomly assigned to one of the two surgeons via a $2 \times 12$ block randomisation list (Sealed Envelope Ltd. 2016). Buprenorphine (0.05 $\mathrm{mg} / \mathrm{kg}$; Buprecare ${ }^{\circledR}$, AST Pharma) and carprofen $\left(4 \mathrm{mg} / \mathrm{kg}\right.$; Norocarp ${ }^{\circledR}$, Norbrook Laboratories) were administered subcutaneously as preoperative analgesia. Anaesthesia was induced using 3-4 vol.\% isoflurane (IsoFlo ${ }^{\circledR}$, Abbott laboratories), and maintained with $2-3$ vol. $\%$. Animals were placed on a heating pad at $38^{\circ} \mathrm{C}$ and their temperature was monitored with a rectal probe. The abdominal fur was removed with electric clippers. The ischaemic button model was applied according to the original publication of Rajab et al. ${ }^{9}$ In short, the technique comprises a midline incision and the creation of four paramedian ischaemic buttons on both sides of the abdominal wall (eight buttons per animal), placed $1 \mathrm{~cm}$ lateral of the midline incision and spaced $1 \mathrm{~cm}$ apart. Each button was created by grabbing a small $5 \mathrm{~mm}$ portion of parietal peritoneum and ligating the base of the tissue with a non-absorbable suture (4-0 Prolene ${ }^{\circledR}$ Ethicon). After tying the suture, it was turned around the entire base of the tissue and tied again to create the ischaemic button. This procedure was repeated for all eight buttons. Thereafter, the abdomen and the skin were closed in the midline by absorbable sutures (4-0 Polyglactin 910 Vycril $^{\circledR}$ Ethicon and 4-0 poliglecaprone 25 Monocryl ${ }^{\circledR}$ Ethicon respectively). The animals were placed in a heated recovery cage (temperature $\approx 36^{\circ} \mathrm{C}$ ) ventilated with $100 \%$ oxygen at $1 \mathrm{~L}$ per minute for postoperative recovery.

The standard analgesia regime was buprenorphine $0.05 \mathrm{mg} / \mathrm{kg}$ administered preoperatively, and buprenorphine $0.03 \mathrm{mg} / \mathrm{kg}$ administered postoperatively, 12 hours after the preoperative analgesia. The morning of the first postoperative day, carprofen 
$4 \mathrm{mg} / \mathrm{kg}$ was given to all animals. Thereafter, analgesia was administered based on clinical signs of distress such as wet-dog shakes, stretching or abnormal body posture, the animal not increasing in weight compared with the previous day or the welfare scores not improving compared with the previous day.

\section{Outcome measurement}

The primary outcome of the study was intra-abdominal adhesion formation. The quantitative analysis of adhesion formation was scored by calculating the amount of intra-abdominal adhesions formed to the number of remaining buttons after 1 week of follow-up (eight buttons per animal - obliterated buttons due to postoperative mobilisation or slipped sutures). Standardised scoring of the adhesion was based on standardised pictures taken by the surgeons (Figure 3.1) and performed by two researchers (EHHM and LH) blinded for sex. Qualitative analysis of the adhesions was performed using the macroscopic Zühlke score (Table 3.1). ${ }^{10}$ Secondary endpoints were welfare scores, postoperative weight gain, need of postoperative analgesia and inter-surgeon variability. Welfare was assessed using a standardised welfare scoring sheet. All animals were scored daily during the full follow-up of the experiment. During the first two postoperative days, an additional 'in cage' observation was performed. Two authors (EHHM and LH) performed each assessment together to reduce interobserver bias. Each welfare assessment comprised of weighing the animal, checking the surgical wound for complications and scoring the following items: activity, behaviour, gait, posture, body condition, fur, hydration, breathing, faeces/urine and mucosa/extremity. Each item was scored 0 (no abnormality), 1 (any deviation from normal), 2 (clearly abnormal) or 3 (very abnormal). In the surgical wound category, a score of 0 reflected normal skin (no wound), 1 indicated a healing wound, 2 indicated a fresh wound and 3 indicated a wound complication.

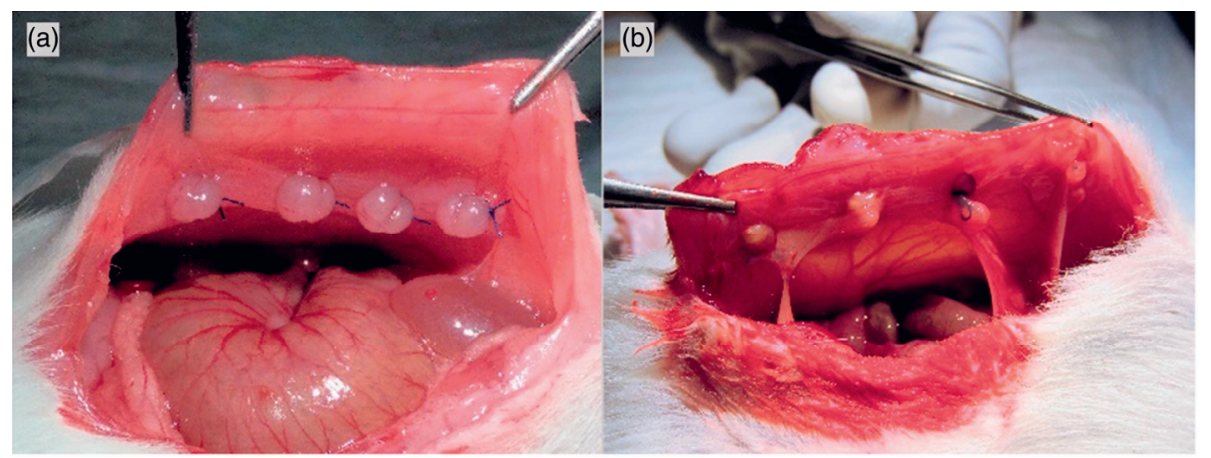

Figure 3.1 Pre- and postoperative view of ischaemic buttons. (a) Preoperative view of four ischaemic buttons; (b) postoperative view of four ischaemic buttons with adhesions to the omentum and epidydimal/peritesticular fat. 
Table 3.1 Zühlke macroscopic adhesion scoring system.

\begin{tabular}{|c|c|}
\hline Grade 0 & No adhesions/ insignificant adhesions \\
\hline Grade 1 & Only one adhesions band between the organs or between one organ and abdominal wall \\
\hline Grade 2 & Two adhesions bands between organs or between one organ and abdom-inal band \\
\hline Grade 3 & $\begin{array}{l}\text { More than two adhesion bands between the organs or between one organ and abdominal wall or } \\
\text { adhesions of intestinal loops without an adhesion to the abdominal wall }\end{array}$ \\
\hline Grade 4 & Adhesions of all viscera to the abdominal wall \\
\hline
\end{tabular}

Source: adapted from Zühlke et al. ${ }^{10}$

A total welfare score was computed by adding all 11 previously mentioned items, and subscores for 'surgical wound', 'behaviour' (items: 'behaviour', 'gait' and 'activity') and 'appearance' (items: 'posture', 'body condition', 'fur' and 'breathing') were computed for a more detailed analysis of welfare. Delta welfare scores were calculated for each of the previously mentioned categories to evaluate the effect of additional analgesia.

\section{Statistical analysis}

Based on an alfa of 0.05 , a beta of 0.20 and an effect size of 2.0, 12 rats were needed in each group. Statistical analysis for the outcomes 'adhesions' and 'welfare/analgesia' were performed using SPSS version 22 (SPSS Statistics for Windows, IBM, Armonk, New York, released 2013). Analysis of weight gain and visual representation of delta weight gain was analysed/ computed by GraphPad Prism ${ }^{\circledR}$, version 6.00 for Windows (GraphPad Software, La Jolla, California; www.graphpad.com). Any group comparison was per-formed by independent student T-tests or Mann-Whitney $U$ tests, depending on the distribution of the data (F-test).

\section{Results}

Two male rats had wound dehiscence in the middle of the laparotomy wound, with an intact underlying fascia. These animals were reoperated on the first postoperative day, the wound was cleaned and the edges of the wound were trimmed until punctate bleeding was observed. Thereafter, the abdomen was closed intracutaneously with 4-0 monocryl ${ }^{\circledR}$ sutures.

\section{Adhesions}

In total, 192 buttons (96 male/96 female) were created during this study. After 1 week of follow-up, 93 buttons survived in the male group (96.9\%) compared with 91 in the female group (94.8\%) $(P=0.409)$. In the male group, 85 out of $93(91.4 \%)$ buttons induced adhesions compared with 84 out of $91(92.3 \%)$ in the female group $(P=0.881)$. 
In the male group, all but one animal were classified as Zühlke type 3; one animal was classified as Zühlke type 4. All female animals were classified as Zühlke type 3 $(P=0.339)$. See Table 3.2 for anatomical structures that adhered to the ischaemic buttons. The number of surviving buttons was equal for both surgeons. Variance in the number of adhesions on the remaining buttons was 1.174 vs. $1.636(P=0.865)$. There was no significant difference between the number of adhesions to the epidydimal or parametrial fat in the lower abdomen $(P=0.730)$.

Table 3.2 Anatomical structures that adhered to the ischemic buttons.

\begin{tabular}{lcc}
\hline & Males & Females \\
\hline Omentum & 41 & 42 \\
Epididymal/ parametrial Fat & 40 & 37 \\
Pancreas & 2 & 3 \\
Liver & 2 & 0 \\
Stomach & 0 & 1 \\
Bladder & 0 & 1 \\
Total & 85 & 84 \\
\hline
\end{tabular}

\section{Welfare/analgesia}

The difference in total welfare scores between male and females was not statistically significant, except for on postoperative day 4. At the fourth day, the mean overall welfare score for the male rats was 7.3 compared with 5.3 in the female group $(P=0.043)$. A higher score indicates more welfare impairment. The difference in welfare score at day 4 is based on a difference in the 'behaviour' subscore at day $4(P=0.012)$. The remaining subscores 'wound' and 'appearance' did not differ statistically significant between male and female rats (Figure 3.2).

All animals received analgesia for the first 48 hours. Thereafter, analgesia was provided based on the welfare scores of an individual animal and/or clinical signs of distress. Additional analgesia was administered nine times (six rats) in the male group and eight times (six rats) in the female group $(P=0.807)$. As mentioned previously, there were two male rats with a reoperation for wound dehiscence, if analgesia within the first 48 hours from reoperation are excluded from the analysis, then there was no statistically significant difference between male and female administration of analgesia $(P=0.797)$. Differences between consecutive days were analysed by calculating delta differences of each category to evaluate the effect of additional analgesia. Only two statistically significant differences were found in the female group at postoperative day 6 between the total welfare $(P=0.02)$ and behavioural scores $(P=0.023)$ in the female group, indicating that the three female rats who received additional analgesia on postoperative day 5 performed significantly better compared with the female rats who did not receive pain medication. Indication for additional analgesia were confirmed by 
analysing the delta differences the day before analgesia was given, which showed statistically significant differences in at least one of the welfare categories at each day, indicating that the rats who received analgesia were not recovering as quickly as the remainder of the group.

(a) Total welfare scores during follow-up

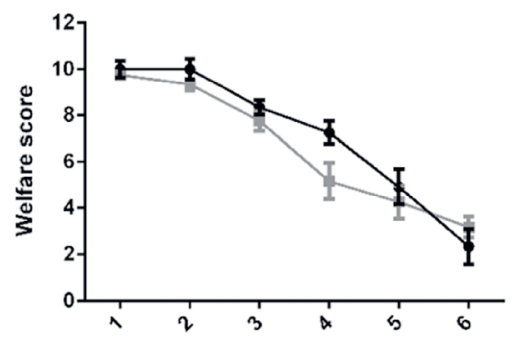

(c) Behaviour scores during follow-up

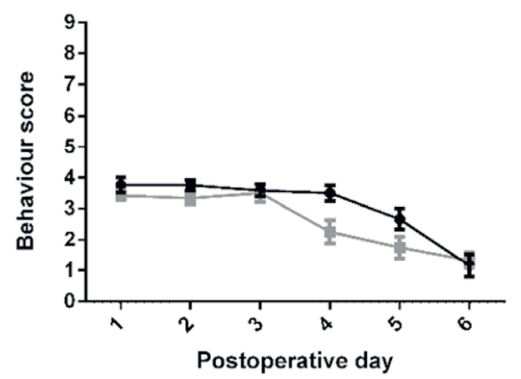

(b) Appearance scores during follow-up

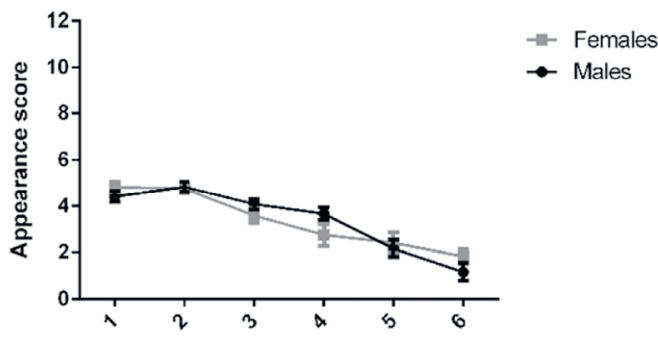

(d) Wound scores during follow-up

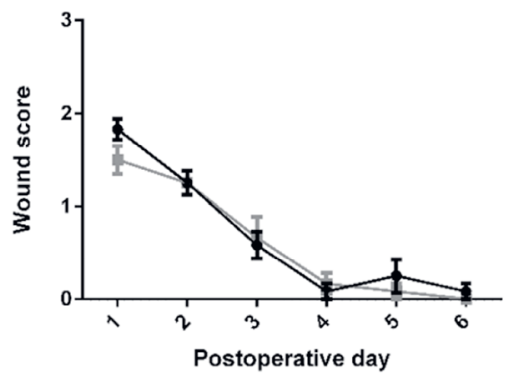

Figure 3.2 Welfare scores during follow-up. Total welfare scores (a), as well as subscores for 'appearance' (b), 'behaviour'.

\section{Weight gain}

Weight was measured daily throughout the follow-up. A delta weight gain was calculated by subtracting the baseline weight of each animal from the measured value to correct for sex-based differences at baseline. Male rats seemed to gain weight significantly faster compared with females (Figure 3.3). At the end of follow-up, $83 \%$ of the male rats had reached or exceeded the preoperative weight compared with $33 \%$ of the female rats. Differences at postoperative days 5-7 are statistically significant (Figure 3.3). 


\section{Delta weight gain during follow-up}

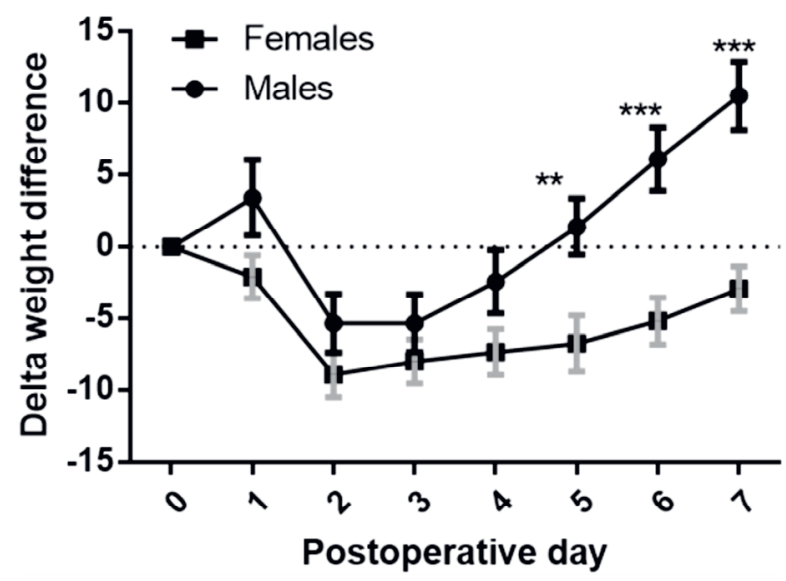

Figure 3.3 Delta weight gain during follow-up for males and females. The dotted line reflects baseline weight. Whiskers show the SEM. Postoperative day 0 is the value at baseline. ${ }^{* *} P<0.01$, $* * * P<0.001$

Figure 3.4 shows the delta weight gain corrected for normal physiological growth. The first 6 postoperative days did not differ significantly. Postoperative day 7 shows a significant difference between males and females $(-10.56 \mathrm{~g}$ vs. $-16.9 \mathrm{~g}$, respectively; $P=0.035$ ).

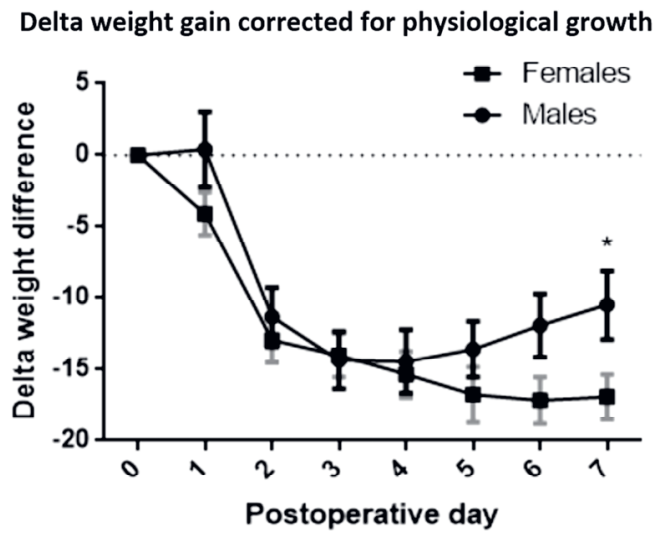

Figure 3.4 Delta weight gain corrected for physiological growth. The dotted line reflects a 'normal' weight for each timepoint based on growth data supplied by Charles River Laboratories concerning German bred Wistar Han rats aged 8 weeks. Whiskers show the SEM. ${ }^{*} P<0.05$; statistical significance on postoperative days $6(p=0.068)$ and $7(p=0.035)$ 
A
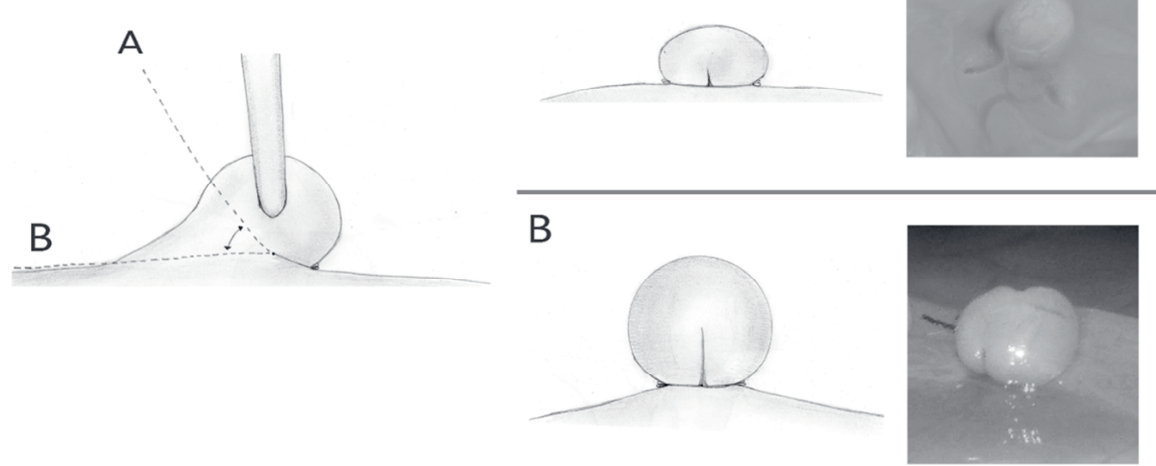

Figure 3.5 Making durable ischaemic buttons. When tying the suture (after completing the backstitch), the angle between the suture and the abdominal wall (double-headed arrow) can vary. A large angle, shown by suture line ' $A$ ', will result in a small button. A small angle, shown by suture line ' $B$ ', will result in a bigger and more durable button.

\section{Discussion}

The ischaemic button model is amongst the most frequently used models for the induction of experimental adhesions. ${ }^{11-15}$ This study shows there is no quantitative nor qualitative difference in the performance of the ischaemic button model between male and female Wistar Han rats. There was a significant difference in welfare on postoperative day 4 , in favour of the female rats, and this difference in total welfare score was the result of a difference in the 'behaviour' subscore on day 4. The average difference was one point on a total score of nine points (behaviour subscore) and was deemed not clinically relevant. There was no statistically significant difference in the need for additional postoperative analgesia. Despite these similarities, male rats reached baseline weight significantly faster compared with female rats. When the delta weight gain was corrected for physiological growth, the statistical significance persisted at the end of the follow-up. The faster weight gain in male rats can be explained by multiple factors, ranging from faster postoperative recovery in the male group to insufficient analgesia schemes in the female group, or unrecognised need for pain medication in the female group. ${ }^{16}$ There is increasing evidence that pain perception has sex-based differences. ${ }^{17}$ Sorge et al. conducted a study in 2015 concerning pain perception in male and female mice, and found sexual dimorphism in pain perception based on Toll-like receptor 4 and dorsal horn gene expression. ${ }^{17,18}$ Previous studies have evaluated the dimorphism effect and demonstrated that opioids 
have a larger analgesic effect in male rats compared with female rats. ${ }^{19,20}$ These findings suggest sexual dimorphism in pain perception, as well as efficacy of analgesia. In our study, additional analgesia during follow-up (carprofen $4 \mathrm{mg} / \mathrm{kg}$ ) was given if the welfare scores of an animal did not improve or the animal showed clear signs of distress. After receiving carprofen, both male and female rats improved clinically.

In this study, approximately one-half of the animals needed additional postoperative pain medication during follow-up despite the pre- and postoperative administration of buprenorphine and carprofen during the first 24 hours, indicating that future studies can and should include a more refined analgesia scheme. Based on the welfare scores of this study, analgesia should be continued for longer than 24 hours postoperatively, for example, 48 or 72 hours.

Button obliteration due to slipping of the suture (3.1\% male group vs $5.2 \%$ female group) in this study was comparable with the study of Rajab et al. that reported a button obliteration of $3.8 \%$, using the modified method. ${ }^{9}$ Adhesion induction of the remaining studies is noticeably higher ( $91.4 \%$ male group vs $92.3 \%$ female group) than previously published work, such as the study of Rajab et al., who reported $62.3 \%$ adhesions in the modified group and $69.2 \%$ adhesions in the original ischaemic button group. However, adhesion induction in this model varies in the published literature from 62.3 to $92 \%{ }^{9,12,13}$ Variation in button obliteration and adhesion induction can be explained by multiple factors such as surgeon performance, suture material, button size, duration of the procedure and duration of follow-up. Button size refers to the amount of tissue protruding over the suture. When tying the back and front ligature of the ischaemic button, it is important to keep the angle between the suture and the abdominal wall as small as possible. This will ensure the creation of a large, durable ischaemic button (Figure 3.5). The type of suture used in the ischaemic button model varies from braided absorbable sutures to monofilament non-absorbable sutures. ${ }^{9,11-15} \mathrm{~A}$ suture comparison for the ischaemic button model has not been performed, although each type of suture has advantages and disadvantages. Braided sutures are easier to tie but are usually thicker. Silk or monofilament sutures are thinner and create a smaller base of the button with profound bulging of the tissue, although they are difficult to tie. Differences in histological response between absorbable and non-absorbable sutures may exist, but are currently based on speculation. ${ }^{21,22}$

The type of tissue adhering to the buttons was comparable in male and female rats. Often male rats are used for this model because of their abundance of epidydimal fat, which is prone to adhere to the buttons. This study demonstrates that there is no difference in the type or location of adhesion induction between male and female rats. Females may have smaller intra-abdominal (parametrial) fat storage but this did not influence proneness to adhesion formation in the lower abdomen. There was no 
significant difference in welfare scores based on the type of organ involved in the adhesion. However, the effector the number of animals may have been too small to detect a difference in welfare scores because of organ involvement, as most animals had adhesions to intra-abdominal fat and only eight animals had adhesions to other organs such as the bladder, gut, liver or pancreas.

This study used an extensive 11-item visual welfare assessment, but this instrument has never been validated. Monitoring welfare in rodents is a difficult task because rodents are prey animals and will try to hide visual indicators for distress if possible. Moreover, behaviours or signs that are scored as signs of distress, such as sluggish movement or decreased food intake, could be a side-effect of the analgesia given. The aim of this study was to perform a clinical comparison of male and female rats in terms of adhesion induction. There were no separate analgesia regimens for male and female rats. Visual welfare assessment was performed directly at the cage, whereas video monitoring of welfare may be less stressful for the animals and therefore be more sensitive to abnormal behaviour.

Welfare in males and females was scored by the same observer to prevent interobserver bias. Postoperative analgesia was standardised during the first postoperative 48 hours, although during the remainder of follow-up, analgesia usage differed between the groups, depending on clinical/welfare assessments. Additional analgesia was given if the total welfare score did not improve compared with the previous day, if the animal had not gained weight compared to the previous day or if the animal showed clear signs of distress (wet-dog shakes, stretching, abnormal body posture, etc.) No fixed cut-off point in welfare scores was defined for providing additional analgesia to the animals. As mentioned previously, the high number of animals needing additional pain medication in this study indicates that future postoperative analgesia protocols should include a longer postoperative analgesia scheme.

After analgesia was given, the welfare scores of the animals improved and showed no statistical differences compared with the other male/female rats that did not receive additional pain medication, with the exception of one timepoint (postoperative day 6 in the female group) that was considered not clinically relevant because the effect was not consistent with observations made on any of the other postoperative days, and therefore was most likely related to the small groups included in this analysis. Given the previously mentioned dimorphism in pain perception and analgesic efficacy of opioids, comparing welfare and pain perception between male and female rats is difficult, and attributing any observed difference in pain perception or analgesia treatment to a single cause is not possible in the current experiment. 


\section{Conclusion}

Despite the discussed limitations, we can draw a conclusion from this study, namely that the qualitative and quantitative performance of the ischaemic button model is equal in male and female Wistar Han rats. In this study, there are no clinically relevant differences in postoperative welfare scores between male and female rats. Male rats seem to gain weight faster during follow-up and reach baseline weight sooner compared with female rats, even if corrected for physiological growth. 


\section{References}

1. Hellebrekers BW, Kooistra T. Pathogenesis of postoperative adhesion formation. Br J Surg. 2011; 98(11):1503-16.

2. Arung W, Meurisse M, Detry O. Pathophysiology and prevention of postoperative peritoneal adhesions. World J Gastroenterol. 2011;17(41):4545-53.

3. Ouaissi M, Gaujoux S, Veyrie N, Deneve E, Brigand C, Castel B, et al. Post-operative adhesions after digestive surgery: their incidence and prevention: review of the literature. J Visc Surg. 2012; 149(2):e104-14.

4. Ellis H, Moran BJ, Thompson JN, Parker MC, Wilson MS, Menzies D, et al. Adhesion-related hospital readmissions after abdominal and pelvic surgery: a retrospective cohort study. Lancet. 1999; 353(9163):1476-80.

5. Wilson MS. Practicalities and costs of adhesions. Colorectal Dis. 2007;9 Suppl 2:60-5.

6. Kossi J, Salminen P, Rantala A, Laato M. Population-based study of the surgical workload and economic impact of bowel obstruction caused by postoperative adhesions. Br J Surg. 2003;90(11): 1441-4.

7. Ray NF, Denton WG, Thamer M, Henderson SC, Perry S. Abdominal adhesiolysis: inpatient care and expenditures in the United States in 1994. J Am Coll Surg. 1998;186(1):1-9.

8. Buckenmaier CC, 3rd, Pusateri AE, Harris RA, Hetz SP. Comparison of antiadhesive treatments using an objective rat model. Am Surg. 1999;65(3):274-82.

9. Rajab TK, Wauschkuhn CA, Smaxwil L, Kraemer B, Wallwiener M, Wallwiener CW. An improved model for the induction of experimental adhesions. J Invest Surg. 2010;23(1):35-9.

10. Zuhlke HV, Lorenz EM, Straub EM, Savvas V. [Pathophysiology and classification of adhesions]. Langenbecks Arch Chir Suppl II Verh Dtsch Ges Chir. 1990:1009-16.

11. Cassidy MR, Sherburne AC, Heydrick SJ, Stucchi AF. Combined intraoperative administration of a histone deacetylase inhibitor and a neurokinin-1 receptor antagonist synergistically reduces intraabdominal adhesion formation in a rat model. Surgery. 2015;157(3):581-9.

12. Kraemer B, Wallwiener C, Rajab TK, Brochhausen C, Wallwiener M, Rothmund R. Standardised models for inducing experimental peritoneal adhesions in female rats. Biomed Res Int. 2014;2014:435056.

13. Sheldon HK, Gainsbury ML, Cassidy MR, Chu DI, Stucchi AF, Becker JM. A sprayable hyaluronate/carboxymethylcellulose adhesion barrier exhibits regional adhesion reduction efficacy and does not impair intestinal healing. J Gastrointest Surg. 2012;16(2):325-33.

14. Vogels RR, Bosmans JW, van Barneveld KW, Verdoold V, van Rijn S, Gijbels MJ, et al. A new poly(1,3-trimethylene carbonate) film provides effective adhesion reduction after major abdominal surgery in a rat model. Surgery. 2015;157(6):1113-20.

15. Wallwiener M, Wallwiener CW, Molinas R, Rajab TK, Brucker SY, Kraemer B, et al. Intraabdominal adhesion formation is associated with differential mRNA expression of metabolic genes PDHb and SDHa. Arch Gynecol Obstet. 2012;286(3):683-6.

16. Waite ME, Tomkovich A, Quinn TL, Schumann AP, Dewberry LS, Totsch SK, et al. Efficacy of Common Analgesics for Postsurgical Pain in Rats. J Am Assoc Lab Anim Sci. 2015;54(4):420-5.

17. Sorge RE, Mapplebeck JC, Rosen S, Beggs S, Taves S, Alexander JK, et al. Different immune cells mediate mechanical pain hypersensitivity in male and female mice. Nat Neurosci. 2015;18(8):1081-3.

18. Sorge RE, LaCroix-Fralish ML, Tuttle AH, Sotocinal SG, Austin JS, Ritchie J, et al. Spinal cord Tolllike receptor 4 mediates inflammatory and neuropathic hypersensitivity in male but not female mice. J Neurosci. 2011;31(43):15450-4.

19. Wang X, Traub RJ, Murphy AZ. Persistent pain model reveals sex difference in morphine potency. Am J Physiol Regul Integr Comp Physiol. 2006;291(2):R300-6.

20. Loyd DR, Wang X, Murphy AZ. Sex differences in micro-opioid receptor expression in the rat midbrain periaqueductal gray are essential for eliciting sex differences in morphine analgesia. $\mathrm{J}$ Neurosci. 2008;28(52):14007-17. 
21. Kharwadkar N, Naique S, Molitor PJ. Prospective randomized trial comparing absorbable and nonabsorbable sutures in open carpal tunnel release. J Hand Surg Br. 2005;30(1):92-5.

22. Sajid MS, Parampalli U, Baig MK, McFall MR. A systematic review on the effectiveness of slowlyabsorbable versus non-absorbable sutures for abdominal fascial closure following laparotomy. Int $\mathbf{J}$ Surg. 2011;9(8):615-25. 

Part II

Repair of fascia in the abdominal wall 



\section{Chapter 4}

\section{Temporal changes in cell populations and gene expression during abdominal wall fascial healing}

Hong Liu, Audrey C.H.M. Jongen, Xinwei Chang, Jarno Melenhorst, Kaatje Lenaerts, Marion J. Gijbels, and Nicole D. Bouvy Molecular Biology, submitted, under revision 


\section{Abstract}

\section{Background}

Few studies concern normal fascial healing process despite it is of importance to prevent incisional hernia. This study aims to reveal the biological process of fascial restoration after surgical incision in rats, evaluating the temporal changes in cell populations and gene expression levels.

\section{Methods and results}

After operations, twenty Wistar underwent a midline laparotomy were sacrificed to collect fascial samples at 4 different time points ( 6 hours, 24 hours, 3 days, and 7 days). Sliced samples stained by haematoxylin and eosin, were blindly evaluated. Gene expression levels were detected by real-time quantitative polymerase chain reaction. Percentage of granulocytes of granulocytes and macrophages decreased significantly between 24 hours and 7 days, and vice versa for macrophages $(P=0.030)$. Fibroblasts increased significantly from 24 hours until 7 days $(P=0.047)$. Gene expression changes were found for $\operatorname{Nos} 2(P=0.031), \operatorname{Mrcl}(P=0.002), C d 163(P=0.028), \operatorname{Vegfa}(P=0.003)$, and Angpt2 $(P=0.014)$ between 6 hours and 3 days; for Clec10a $(P=0.020)$ between 24 hours and 3 days; for Nos2 $(P=0.004)$, Vegfa $(P=0.017)$, and Ptgs $2(P=0.002)$ between 6 hours and 7 days; and for $C d 163(P=0.028)$ between 3 days and 7 days.

\section{Conclusions}

Granulocytes and macrophages predominate in the early and following inflammatory phase of fascial healing, respectively. Fibroblasts appear from the middle of the first postoperative week. Macrophage polarization in fascial healing may be activated very early postsurgery. 


\section{Introduction}

Incisional hernia (IH) is one of the most common complications after laparotomy. The incidence of $\mathrm{IH}$ following midline incision is around $13 \%$ within two postoperative years, ${ }^{1}$ and associated costs are estimated to be billions of Euros for the European Union every year. ${ }^{2,3}$ Generally, IH is defined as a gap or fascial defect in the area of abdominal wall scar that can be detected by clinical examination or imaging. ${ }^{4}$ Separation of injured fascia after a closure frequently happens in the early postoperative period, resulting in clinical detection of $\mathrm{IH}$ from the first postoperative month onwards. ${ }^{5}$

Proper wound healing is an essential process in response to tissue damage after acute injury, such as during a surgical intervention. Fasica, virtually surrounding all other structures and organs, is a distinctive organ providing function of stability and motion. ${ }^{6,7}$ Numerous studies have focused on failed fascial healing and the formation of $\mathrm{IH}$, whereas the normal process of fascial healing of the abdominal wall after surgery is poorly studied. ${ }^{8,9}$ Furthermore, most previous IH animal models concern changes in the healing process after one week. There is a paucity of evidence regarding the healing process in the very early postoperative period. Temporal series of the healing changes are especially lacking. Radu et al. have reviewed human trials, showing that collagen disorders mainly contribute to the formation of IH. ${ }^{10}$ Jansen et al. have illustrated that macrophages, fibroblasts, and their released proteases and cytokines may be involved in the IH formation. ${ }^{8}$ Fibroblast activities, including migration, multiplication, and collagen production, play a critical role in fascial regeneration sustaining the mechanical tension. ${ }^{11-13}$ Skin wound healing is widely studied and macrophage polarization makes important contributions to this process, in which local macrophages predominate from classically activated macrophages (M1-like phenotypes) to alternative activated macrophages (M2-like phenotypes). M1-like macrophages are pro-inflammatory whereas M2-like phenotypes are anti-inflammatory and proregenerate. ${ }^{14,15}$

The current study aims to explore the biological process of normal fascial healing, focusing on the temporal changes of both the cell population and the gene expression level in rats. Due to the difficulty isolating functionally healed fascia from patients after laparotomy, animal models are used to mimic this clinical setting. Once we have unravelled the process of fascial healing under physiological circumstances, we can induce regeneration of fascia by adding certain substances to improve fascial healing and prevent IH formation in the future. 


\section{Methods}

\section{In vivo experiment}

\section{Ethics}

The Dutch Animal Experimental Committee approved the animal experiment. All procedures on animals complied with the guidelines of the Dutch Animal Experimental Act and were approved by the Animal Experimental Committee of Maastricht University. Data reporting of this experiment followed the ARRIVE (Animal Research: Reporting of In Vivo Experiments) guidelines.

\section{Housing conditions}

Adult male Wistar rats, weighing 250-300 g, were obtained from Harlan Company. All animal procedures were conducted at the animal centre of Maastricht University. Animals were acclimatized one week before surgery. Two animals were housed in one Macro cage (EU type IV, $1800 \mathrm{~cm}^{2}$ ) filled with hygiene animal bedding with enrichments of cardboard and wooden block, located in an animal housing room fitted with 12-hour light-night circle and $25^{\circ} \mathrm{C}$ temperature. Animals were allowed to access food (10 mm Sniff rat/mouse sterilized food compressed into pellets) and drink (acidified water) ad libitum. Surgeries were performed in a standard operation room with anaesthesia systems and monitoring devices, separated from the animal housing room.

\section{Grouping}

In this study, 20 animals, served as controls in an experiment investigating the anastomotic healing process, were included. These animals underwent open surgery for intestinal anastomosis without any other interventions and did not show anastomotic leakage nor impaired healing. In that experiment, animals were randomly allocated into four groups with five animals per group: 6 hours, 24 hours, 3 days, and 7 days postsurgery. One sample of fascia was collected from each rat for analysis.

\section{Surgical procedures}

Pain relief was administered subcutaneously to all animals using buprenorphine $0.05 \mathrm{mg} / \mathrm{kg}$ prior to surgery. Anaesthesia of animals was induced by inhalation of $4 \%$ isoflurane and then maintained with $2 \%$ isoflurane. After shaving, the animals were placed on a heated mat. Temperature and heartbeat of the animals were monitored with a rectal probe. The abdominal area was disinfected with a $2 \%$ iodine solution and covered with sterile drapes except for the operation area. A middle line abdominal incision of around $5 \mathrm{~cm}$ was performed under aseptic conditions. After the abdominal 
wall was incised layer by layer, the wound was protected by sterile gauze wetted with $0.9 \%$ sodium chloride solution. Caecum was exposed and placed on the wet gauze outside of the peritoneal cavity. During the entire operation, the caecum and other intestines located outside remained wet surrounded by wet gauzes. Ascending colon was transversely cut off at two centimetres from the ileocecal angle. Stool was removed by moist sterile gauzes. The two ends of the colon were then interruptedly closed together with 12 equidistant stitches using Prolene ${ }^{\circledR}$ 6-0 sutures (Ethicon Inc; Johnson\&Johnson, Somerville, NJ). The fascia was closed continuously by Vicyl ${ }^{\mathrm{TM}}$ 4-0 (Ethicon Inc; Johnson\&Johnson, Somerville, NJ) suture and the skin was closed intracutaneously with Monocryl ${ }^{\mathrm{TM}}$ 4-0 (Ethicon Inc; Johnson\&Johnson, Somerville, NJ).

The animals were allowed to return to a clean cage with the identical companion after recovery from the anaesthesia. Animals had ad libitum access to food and water during postoperative days. Animals were examined daily based on an evaluation form with multiple items (weight, temperature, activity, behaviour, gait, posture, physical condition, fur/skin, hydration, breathing, faeces/urine, surgical wound, oedema, and necrosis). Buprenorphine $0.05 \mathrm{mg} / \mathrm{kg}$ was given subcutaneously to all animals in the first two postoperative days.

All animals were sacrificed on the dates according to the group they were allocated to. The sacrifice was conducted via cardiac puncture with complete blood drawing under anaesthesia and then cervical dislocation. Fascial samples (including fascial incision, sutures and surrounded muscles) were collected with a snap freezing technique using liquid nitrogen. Afterwards, samples were stored at $-80^{\circ} \mathrm{C}$.

\section{Histological analyses}

Samples were cut into 10um sections and stained by haematoxylin and eosin (HE) and scored by an experienced pathologist of Maastricht University (M.G.) who was blinded for histological evaluations. Fibroblast score, percentage of granulocytes, percentage of macrophages, and a ratio of granulocytes and macrophages were assessed microscopically. A $0-5$ scale described fibroblast scores (0, not present; 1 , slightly present; 2 , more than slightly present; 3 , moderately present; 4 , more than moderately present; 5, abundantly present). Percentage of granulocytes or macrophages was calculated as follows (granulocytes or macrophages) $\div$ (granulocytes + macrophages $) \times$ $100 \%$. 


\section{RNA isolation, cDNA synthesis, and qPCR}

RNA was isolated by TRI Reagent (Sigma, NL) from tissues. cDNA was synthesized from 15 ul (50 ng/ul) RNA using cDNA synthesis kit (SensiFAST ${ }^{\mathrm{TM}}$, Bioline, London, UK). qPCR was performed using LightCycler $480^{\circledR}$ instrument II (Roche Molecular Systems, Inc.) with a mix of $4.5 \mathrm{ul} \mathrm{cDNA}, 5 \mathrm{ul} \mathrm{SYBR}$ (SensiFAST ${ }^{\mathrm{TM}} \mathrm{SYBR}^{\circledR}$ Hi-ROX Kit, Bioline, London, UK), and 0.5 ul primer (Sigma, NL). Gene expression levels were analysed by LinRegPCR software. Gene $36 b 4$ was used as a housekeeping gene and normalisation factor. Primers were designed based on literature ${ }^{16}$ and a Pubmed tool (Primer-blast). A total of 13 genes were tested including Nos2, Cd86, Clec10a, Mrc1, Cd163, Tgfb1, Colla1, Mmp1, Fn1, Vegfa, Angpt2, Nos3, and Ptgs2. Primers for these genes are shown in Table 4.1.

Table 4.1 Primers of all tested genes and possible functions of all these genes.

\begin{tabular}{|c|c|c|}
\hline Gene & $\begin{array}{l}\text { Related cell type / } \\
\text { process }\end{array}$ & Primer \\
\hline $36 b 4$ & \multirow{2}{*}{ Housekeeping gene } & F-5'CCTCACCGAGATTAGGGACA-3' \\
\hline $\begin{array}{l}\text { (ribosomal protein lateral stalk } \\
\text { subunit P0) }\end{array}$ & & R-5’ATCGCTCAGGATTTCAATGG-3' \\
\hline Nos 2 & \multirow[t]{2}{*}{ M1 macrophage } & F-5' TAGTCAACTACAAGCCCCACG-3' \\
\hline (nitric oxide synthase 2 ) & & R-5’GTGAGGAACTGGGGGAAACC-3’' \\
\hline Cd86 & \multirow[t]{2}{*}{ M1/M2b macrophage } & F-5'AGACATGTGTAACCTGCACCAT-3' \\
\hline (CD86 molecule) & & R-5’TACGAGCTCACTCGGGCTTA-3’' \\
\hline Ptgs 2 & \multirow[t]{2}{*}{ M1 macrophage } & F-5'CTCAGCCATGCAGCAAATCC-3’ \\
\hline $\begin{array}{l}\text { (prostaglandin-endoperoxide } \\
\text { synthase 2) }\end{array}$ & & R-5’ GGGTGGGCTTCAGCAGTAAT-3' \\
\hline Clec10a & \multirow[t]{2}{*}{ M2 macrophage } & F-5'GAGGCTTGAGCCAGAAGGTG-3' \\
\hline $\begin{array}{l}\text { (C-type lectin domain containing } \\
10 \mathrm{~A})\end{array}$ & & R-5'TGCTGAGCCGTTGTTCTTGAG-3' \\
\hline Mrcl & \multirow[t]{2}{*}{ M2 macrophage } & F-5'CCCGCTCCTCAAGACAATCC-3' \\
\hline (mannose receptor, $\mathrm{C}$ type 1 ) & & R-5'AAATACGGTGACTGCCCACC-3' \\
\hline Cd163 & \multirow[t]{2}{*}{ M2 macrophage } & F-5' CTCTGAAGCGACGACAGACC-3' \\
\hline (CD163 molecule) & & R-5’ATGCCAACCCGAGGATTTCA-3' \\
\hline $\operatorname{Tg} f b 1$ & \multirow[t]{2}{*}{ M2 macrophage } & F-5' GGCTGAACCAAGGAGACGGA-3' \\
\hline (transforming growth factor, beta 1) & & R-5’ CCTCGACGTTTGGGACTGAT-3’ \\
\hline Collal & \multirow[t]{2}{*}{ Fibroblast } & F-5' CTGACTGGAAGAGCGGAGAG-3' \\
\hline (collagen type I alpha 1 chain) & & R-5' CAGGATCGGAACCTTCGCTT-3’' \\
\hline Mmpl & \multirow[t]{2}{*}{ Fibroblast } & F-5'AAGGCCACTGGTGATCTTGC-3' \\
\hline (matrix metallopeptidase 1) & & R-5'GGTATTTCCAGACTGTTTCCACA-3' \\
\hline \multirow[t]{2}{*}{ Fnl (fibronectin 1) } & \multirow[t]{2}{*}{ Fibroblast } & F-5’ TCCCCTCCCAGAGAAGTGG-3’ \\
\hline & & R-5'TTGGGGAAGCTCATCTGTCTTTT-3' \\
\hline$V e g f a$ & \multirow{2}{*}{ Vascularisation } & F-5'AGAAGGGGAGCAGAAAGCCC-3' \\
\hline (vascular endothelial growth factor A) & & R-5'GATCCGCATGATCTGCATAGTGA-3' \\
\hline Angpt 2 & \multirow[t]{2}{*}{ Vascularisation } & F-5’CATGATGTCATCGCCCGACT-3’' \\
\hline (angiopoietin 2) & & R-5'TCCATGTCACAGTAGGCCTTG-3' \\
\hline Nos 3 & \multirow[t]{2}{*}{ Vascularisation } & F-5'GAATGGAGAGAGCTTTGCAGC-3' \\
\hline (nitric oxide synthase 3 ) & & R-5'CCGCCAAGAGGATACCAGTG-3' \\
\hline
\end{tabular}




\section{Statistics}

All groups were compared with each other with a total of six comparisons. qPCR data were presented as mean \pm standard deviation (SD) and histological results were present as median \pm interquartile range. Normal distribution of data was not checked due to the small sample size. ${ }^{17}$ The Kruskal-Wallis test was used for all comparisons identifying the differences between time points. Adjusted $P<0.05$ represented significant difference.

\section{Results}

\section{Macroscopic observation in animals}

All animals in the four groups recovered without any complications including no anastomotic leakage and no wound infection. No obvious defect of the fascia was observed macroscopically in any of the animals during sacrifice.

\section{Histological outcomes}

A total of 20 samples were available for the evaluation of fibroblast score. The granulocytes and macrophages were detectable in 18 out of the 20 samples. In one 6-hours sample and one 7-days sample, granulocytes and macrophages were not detected. Fibroblasts were detectable in 19 out of 20 samples with one 7-days sample undetected. Fibroblasts appeared from 3 days post-surgery and increased significantly from 24 hours to the highest level at 7 days post-surgery $(P=0.047)$. Granulocytes comprised more than $50 \%$ of the granulocytes and macrophages at 6 hours and 24 hours. Thereafter, macrophages composed more than $50 \%$ of the granulocytes and macrophages. A significant difference of granulocyte score and macrophage score among the four groups was only detected between the 24 hours group and the 7 days group $(P=0.030)$. Histological scores and typical images are illustrated in Figure 4.1. 


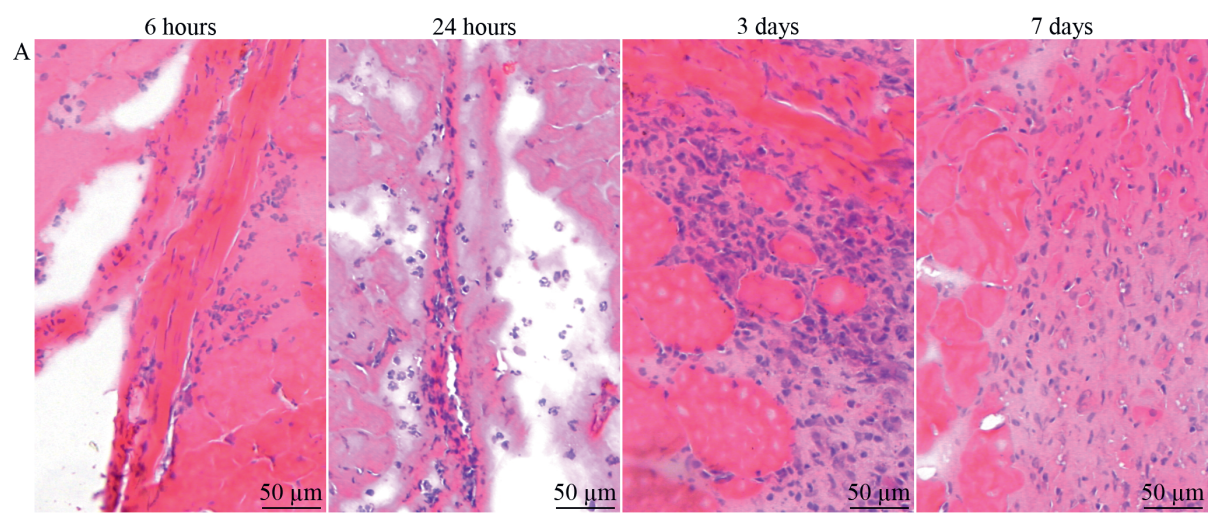

B
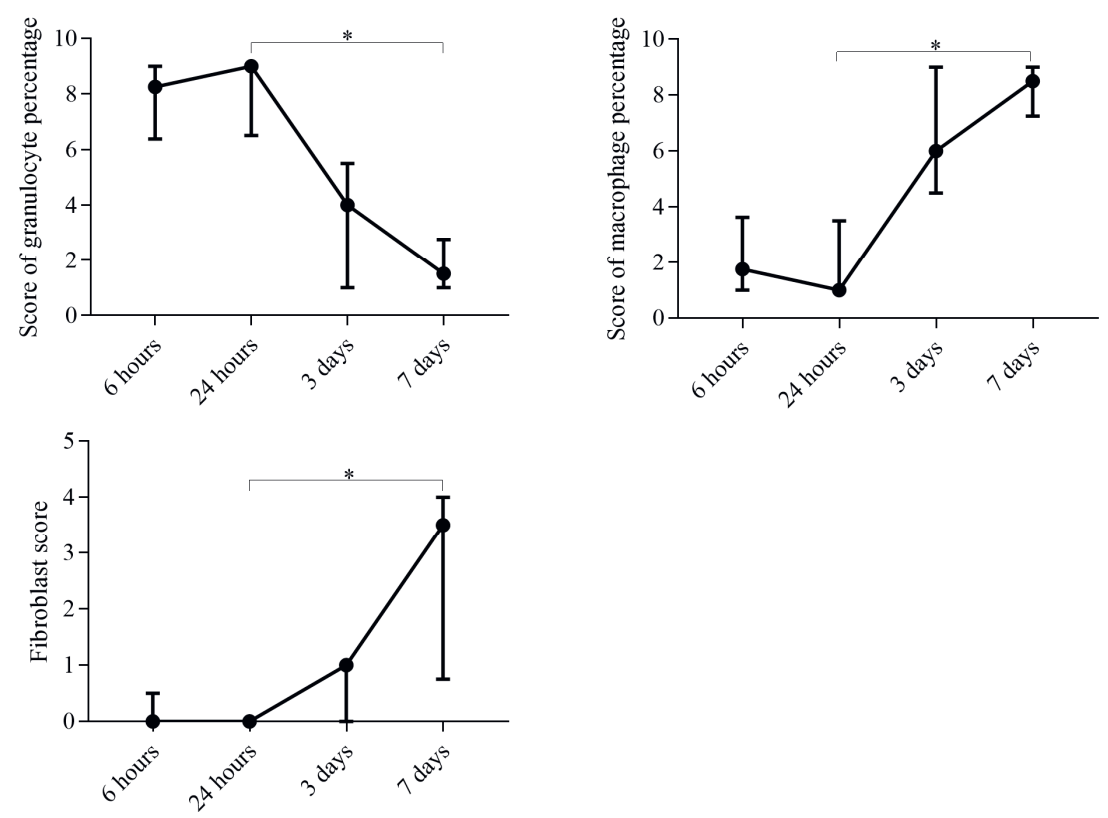

Figure 4.1 Hematoxylin \& eosin (H\&E) staining of midline fascia at four different time points postsurgery. Histological scores of granulocyte percentage, macrophage percentage, and fibroblasts (B) with representative H\&E images focusing on fascial incision area at 6 hours, 24 hours, 3 days, and 7 days (A). The scale bar is 50-micrometer. All data of scores are presented with median \pm interquartile range and analysed with Kruskal-Wallis test to find timedependent differences. $*$ adjusted $P<0.05$. 


\section{Differences in gene expression levels between groups}

Fibroblast activity-related genes, including Collal, Mmpl, and Fn1, did not show significant expression differences between time points after surgery.

Genes related to macrophage growth, including Nos2, Cd86, Clec10a, Mrc1, Tgfb1, Cd163, and Ptgs2, were assessed. Both Cd86 and Tgfb did not differ significantly among time points. The expression level of Nos 2 at 6 hours was significantly higher than that at 3 days $(P=0.031)$ and 7 days $(P=0.004)$. Difference for Clec10a was only detected between 24 hours and 3 days post-surgery $(P=0.020)$. Mrcl was significantly higher expressed at 3 days in comparison with 6 hours post-surgery $(P=0.002)$. Both Clec $10 a$ and $\mathrm{Mrcl}$ showed the maximal expression at 3 days post-surgery. Cd163 showed the highest expression at 3 days post-surgery, with significant differences compared to 6 hours $(P=0.028)$ and 7 days post-surgery $(P=0.028)$. Ptgs 2 showed the highest expression at 6 hours, and was significantly different from 7 days post-surgery $(P=0.002)$.

Vegfa, Angpt2, and Nos3 were as markers for vascularisation. Gene expression of Vegfa was significantly lower at 3 days $(P=0.003)$ and 7 days $(P=0.017)$ compared to 6 hours post-surgery. Expression of Angpt 2 was significantly different between 6 hours and 3 days post-surgery $(P=0.014)$. Nos 3 did not show a significant difference over time. Differentially expressed genes over time are displayed in Figure 4.2. 

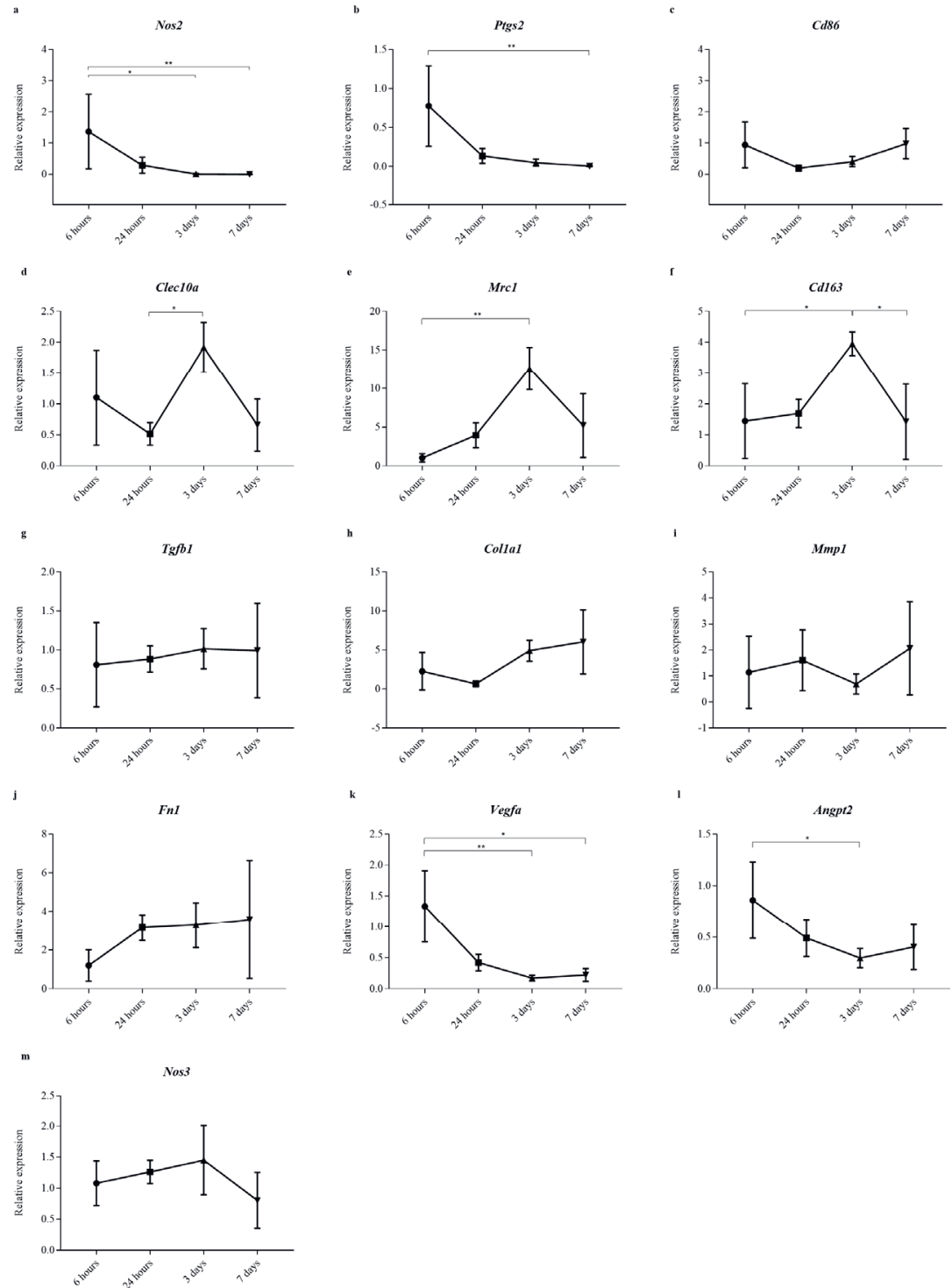

Figure 4.2 Gene expression level of Nos2 (a), Ptgs2 (b), Cd86 (c), Clec10a (d), Cd163 (f), Mrc1 (e), Tgfb1 (g), Col1a1 (h), Mmp1 (i), Fn1 (j), Vegfa (k), Angpt2 (l), and Nos3 (m) at four different time points post-surgery. Data (presented as mean $+\mathrm{SD}$ ) were analysed with Kruskal-Wallis test to find time-dependent differences; * adjusted $P<0.05$; ** adjusted $P<0.01$. 


\section{Discussion}

The biological process of fascial healing after abdominal surgery remains poorly understood. This study evaluated the temporal changes in cell populations as well as the changes in gene expression related to macrophage polarization, fibroblast activity, and vascularization, revealing normal fascial healing process in the first postoperative week.

Cells are the foundation of the regenerated fascia. In guinea pigs, Ross and Benditt have measured cell population of abdominal fascia after surgery, showing that first neutrophils dominate the early stages of inflammation, followed by macrophages, and a little later fibroblasts and lymphocytes. ${ }^{18}$ According to our results in rats, granulocytes and macrophages were recruited to heal fascia after abdominal surgery in the first postoperative week. Granulocytes comprised the main inflammatory cells of granulocytes and macrophages in the first days while macrophages replaced granulocytes as the main inflammatory cells in the following days. Fibroblasts began to appear from the middle of the first postsurgery week and increased significantly at the end of the first week. Our findings in rats are consistent with the previous study in the guinea pigs. Furthermore, the healing of fascia regarding the cell types predominated in inflammatory cells and the changes of fibroblasts as well as the related time course seems comparable to the wound healing of $\operatorname{skin}^{19,20}$ despite belonged to different organs.

Macrophage polarization may be activated very early postsurgery in fascial healing according to our results. Nos 2 and Ptgs 2, gene markers for M1-like macrophages, ${ }^{21}$ are expressed at the highest at level at 6 hours after which expression decreased. In contrast, expression of Clec10a, Mrcl, and Cd163, as markers of M2-like macrophage, ${ }^{22-24}$ increased to the highest level at 3 days. This may indicate the macrophage polarization starts before day 3 postoperatively. Cd86 did not show any significant change over time. Cd86 expression did not change over time, which might be explained by the fact that $C d 86$ is a marker for both M1-like macrophages and M2like macrophages. ${ }^{25,26}$ TGF- $\beta 1$ plays an important role in normal skin wound healing, rapidly increasing after injury and reaching a peak at 3 days post-injury. ${ }^{27}$ On one hand, endogenous TGF- $\beta 1$ overexpression accelerates healing process of partial-thickness wound (epidermis only) but delays healing process of full-thickness wound. ${ }^{27,28}$ On the other hand, the deficiency of TGF- $\beta 1$ delays the full-thickness wound healing. In our results in fascial incision, rats healed normally at 7 days and $T g f b 1$ expression level did not change significantly in the first week, suggesting the normal expression level of $T g f b l$ at a stable level in normal fascial healing. Therefore, compared to superficial skin wound, $T g f b 1$ as a therapeutic target for fascial healing needs further investigation. 
Fibroblast activities, measured by assessing gene expression levels of Colla1, Mmp1, and $F n 1,{ }^{29-32}$ were not significantly changed over time in the first postoperative week. Fibroblast numbers, however, appeared from 3 days and significantly increased from 24 hours to 7 days. This may indicate that Collal, Mmpl, and Fnl expressing consistently are not affected by the changes of the cells in the first postoperative week.

Deep fascia was thought to be essentially avascular, but more studies have reported that deep fascia contain many capillaries. ${ }^{33}$ Lobov and Lee have demonstrated that Angpt2 and Vegfa promote angiogenesis ${ }^{34}$ and Nos3 facilitates skin wound healing in mice. ${ }^{35}$ However, hypoxia and/or inflammation can also induce Angpt2 and Vegfa. In current study, Angpt2 and Vegfa were expressed at the highest level at 6 hours followed by significant decreases over time, and expression of Nos 3 did not change during the first week postsurgery. The high expression level of Angpt2 and Vegfa in such early phase might be caused by hypoxia and/or inflammation due to the fascia injury, aimed at inducing angiogenesis.

HIF-1 signalling pathway has been extensively studied in processes of skin wound healing, due to the critical role of HIF-1 in virtually all the healing processes. ${ }^{36,37}$ Neither abdominal healing of fascia nor IH has been connected to this signalling pathway in previous studies. In the present study, Nos2, Cd163, Mrc1, Vegfa, and Angpt 2 were differentially expressed between 6 hours and 3 days. Three (Nos2, Vegfa, and Angpt2) out of the five genes were involved in the HIF-1 signalling pathway, all related to the increase of oxygen delivery. Previous studies have illustrated that HIF-1 $\alpha$ has influence on the development of $\mathrm{IH}^{38}$ Therefore, HIF-1 pathway might be involved in the process of fascial healing. Possibly not only the HIF-1 signalling pathway but also other signalling pathways might be involved in fascial healing, but this would require further investigation.

There are some limitations to this study. Firstly, it is an animal study, of which results may only be partly translatable to humans. Despite the difference between animals and humans, van den Hil et al. have demonstrated that histological outcomes are comparable between rats and humans in IH research. ${ }^{39}$ In previous wound scar studies, rats show comparability to humans at microscopic and gene expression levels. ${ }^{40,41}$ Secondly, only four time points within the first week were studied. Longer follow-up and more time points will provide a more complete picture of the fascial healing process. Importantly however, if we want to influence fascial healing we should probably focus on stimulating the regeneration processes within the first days after surgery. Lastly, limited genes were analyzed. Nevertheless, this study primarily revealed the basic biological processes of fascial healing. Based on these findings, further research on the regulation mechanism of fascial healing and possible compounds of accelerating fascial healing is clearly demanded. 


\section{Conclusions}

Granulocytes predominate in the fascial cell population first and then are altered by macrophages from 3 days post-surgery onwards. Fibroblasts appear from the middle of the first postoperative week. Gene expression indicates that macrophage polarization in normal fascial healing is activated very early postsurgery. 


\section{References}

1. Bosanquet DC, Ansell J, Abdelrahman T, Cornish J, Harries R, Stimpson A, et al. Systematic Review and Meta-Regression of Factors Affecting Midline Incisional Hernia Rates: Analysis of 14,618 Patients. PLoS One. 2015;10(9):e0138745.

2. Gillion JF, Sanders D, Miserez M, Muysoms F. The economic burden of incisional ventral hernia repair: a multicentric cost analysis. Hernia. 2016;20(6):819-30.

3. Poulose BK, Shelton J, Phillips S, Moore D, Nealon W, Penson D, et al. Epidemiology and cost of ventral hernia repair: making the case for hernia research. Hernia. 2012;16(2):179-83.

4. Sanders DL, Kingsnorth AN. The modern management of incisional hernias. BMJ. 2012;344:e2843.

5. Pollock AV, Evans M. Early Prediction of Late Incisional Hernias. Brit J Surg. 1989;76(9):953-4.

6. Kumka M, Bonar J. Fascia: a morphological description and classification system based on a literature review. J Can Chiropr Assoc. 2012;56(3):179-91.

7. Stecco C, Macchi V, Porzionato A, Duparc F, De Caro R. The fascia: the forgotten structure. Ital J Anat Embryol. 2011;116(3):127-38.

8. Jansen PL, Mertens Pr P, Klinge U, Schumpelick V. The biology of hernia formation. Surgery. 2004;136(1):1-4.

9. Lau FH, Pomahac B. Wound healing in acutely injured fascia. Wound Repair Regen. 2014;22 Suppl $1: 14-7$.

10. Radu P, Bratucu M, Garofil D, Goleanu V, Popa F, Strambu V. The Role of Collagen Metabolism in the Formation and Relapse of Incisional Hernia. Chirurgia (Bucur). 2015;110(3):224-30.

11. Bordoni B, Zanier E. Understanding Fibroblasts in Order to Comprehend the Osteopathic Treatment of the Fascia. Evid Based Complement Alternat Med. 2015;2015:860934.

12. Liptan GL. Fascia: A missing link in our understanding of the pathology of fibromyalgia. J Bodyw Mov Ther. 2010;14(1):3-12.

13. Muir IFK. Control of fibroblast activity in scars: a review. Eur J Plast Surg (21):1-7.

14. Mills CD, Kincaid K, Alt JM, Heilman MJ, Hill AM. M-1/M-2 macrophages and the Th1/Th2 paradigm. J Immunol. 2000;164(12):6166-73.

15. Krzyszczyk P, Schloss R, Palmer A, Berthiaume F. The Role of Macrophages in Acute and Chronic Wound Healing and Interventions to Promote Pro-wound Healing Phenotypes. Front Physiol. 2018; 9:419.

16. van Steensel S, van den Hil LCL, Bloemen A, Gijbels MJ, Breukink SO, Melenhorst J, et al. Prevention of incisional hernia using different suture materials for closing the abdominal wall: a comparison of PDS, Vicryl and Prolene in a rat model. Hernia. 2019.

17. Patricio M, Ferreira F, Oliveiros B, Caramelo F. Comparing the performance of normality tests with ROC analysis and confidence intervals. Commun Stat-Simul C. 2017;46(10):7535-51.

18. Ross R, Benditt EP. Wound healing and collagen formation. I. Sequential changes in components of guinea pig skin wounds observed in the electron microscope. J Biophys Biochem Cytol. 1961;11: 677-700.

19. Gonzalez AC, Costa TF, Andrade ZA, Medrado AR. Wound healing - A literature review. An Bras Dermatol. 2016;91(5):614-20.

20. Canedo-Dorantes L, Canedo-Ayala M. Skin Acute Wound Healing: A Comprehensive Review. Int J Inflam. 2019;2019:3706315.

21. Koh YC, Yang G, Lai CS, Weerawatanakorn M, Pan MH. Chemopreventive Effects of Phytochemicals and Medicines on M1/M2 Polarized Macrophage Role in Inflammation-Related Diseases. Int J Mol Sci. 2018;19(8).

22. Zuo Q, Wang SC, Yu XK, Chao WW. Response of macrophages in rat skeletal muscle after eccentric exercise. Chin J Traumatol. 2018;21(2):88-95.

23. Jablonski KA, Amici SA, Webb LM, Ruiz-Rosado Jde D, Popovich PG, Partida-Sanchez S, et al. Novel Markers to Delineate Murine M1 and M2 Macrophages. PLoS One. 2015;10(12):e0145342. 
24. Yu E, Goto M, Ueta H, Kitazawa Y, Sawanobori Y, Kariya T, et al. Expression of area-specific M2macrophage phenotype by recruited rat monocytes in duct-ligation pancreatitis. Histochem Cell Biol. 2016;145(6):659-73.

25. Graff JW, Dickson AM, Clay G, McCaffrey AP, Wilson ME. Identifying functional microRNAs in macrophages with polarized phenotypes. J Biol Chem. 2012;287(26):21816-25.

26. Rojas J, Salazar J, Martinez MS, Palmar J, Bautista J, Chavez-Castillo M, et al. Macrophage Heterogeneity and Plasticity: Impact of Macrophage Biomarkers on Atherosclerosis. Scientifica (Cairo). 2015;2015:851252.

27. Wang XJ, Han G, Owens P, Siddiqui Y, Li AG. Role of TGF beta-mediated inflammation in cutaneous wound healing. J Investig Dermatol Symp Proc. 2006;11(1):112-7.

28. Pakyari M, Farrokhi A, Maharlooei MK, Ghahary A. Critical Role of Transforming Growth Factor Beta in Different Phases of Wound Healing. Adv Wound Care (New Rochelle). 2013;2(5):215-24.

29. Xie T, Wang Y, Deng N, Huang G, Taghavifar F, Geng Y, et al. Single-Cell Deconvolution of Fibroblast Heterogeneity in Mouse Pulmonary Fibrosis. Cell Rep. 2018;22(13):3625-40.

30. Saitta B, Gaidarova S, Cicchillitti L, Jimenez SA. CCAAT binding transcription factor binds and regulates human COL1A1 promoter activity in human dermal fibroblasts: demonstration of increased binding in systemic sclerosis fibroblasts. Arthritis Rheum. 2000;43(10):2219-29.

31. Lindner D, Zietsch C, Becher PM, Schulze K, Schultheiss HP, Tschope C, et al. Differential expression of matrix metalloproteases in human fibroblasts with different origins. Biochem Res Int. 2012;2012: 875742 .

32. Xia W, Hammerberg C, Li Y, He T, Quan T, Voorhees JJ, et al. Expression of catalytically active matrix metalloproteinase-1 in dermal fibroblasts induces collagen fragmentation and functional alterations that resemble aged human skin. Aging Cell. 2013;12(4):661-71.

33. Bhattacharya V, Barooah PS, Nag TC, Chaudhuri GR, Bhattacharya S. Detail microscopic analysis of deep fascia of lower limb and its surgical implication. Indian J Plast Surg. 2010;43(2):135-40.

34. Lobov IB, Brooks PC, Lang RA. Angiopoietin-2 displays VEGF-dependent modulation of capillary structure and endothelial cell survival in vivo. Proc Natl Acad Sci U S A. 2002;99(17):11205-10.

35. Lee PC, Salyapongse AN, Bragdon GA, Shears LL, 2nd, Watkins SC, Edington HD, et al. Impaired wound healing and angiogenesis in eNOS-deficient mice. Am J Physiol. 1999;277(4):H1600-8.

36. Heun Y, Pogoda K, Anton M, Pircher J, Pfeifer A, Woernle M, et al. HIF-1alpha Dependent Wound Healing Angiogenesis In Vivo Can Be Controlled by Site-Specific Lentiviral Magnetic Targeting of SHP-2. Mol Ther. 2017;25(7):1616-27.

37. Ruthenborg RJ, Ban JJ, Wazir A, Takeda N, Kim JW. Regulation of Wound Healing and Fibrosis by Hypoxia and Hypoxia-Inducible Factor-1. Molecules and Cells. 2014;37(9):637-43.

38. Thankam FG, Palanikumar G, Fitzgibbons RJ, Agrawal DK. Molecular Mechanisms and Potential Therapeutic Targets in Incisional Hernia. J Surg Res. 2019;236:134-43.

39. van den Hil LCL, Vogels RRM, van Barneveld KWY, Gijbels MJJ, Peutz-Kootstra CJ, Cleutjens JPM, et al. Comparability of histological outcomes in rats and humans in a hernia model. J Surg Res. 2018;229:271-6.

40. Occleston NL, Metcalfe AD, Boanas A, Burgoyne NJ, Nield K, O'Kane S, et al. Therapeutic improvement of scarring: mechanisms of scarless and scar-forming healing and approaches to the discovery of new treatments. Dermatol Res Pract. 2010;2010.

41. Grada A, Mervis J, Falanga V. Research Techniques Made Simple: Animal Models of Wound Healing. J Invest Dermatol. 2018;138(10):2095-105 e1. 



\title{
Chapter 5
}

\begin{abstract}
Abdominal fascial healing in the first postoperative week: a MALDI-TOF mass spectrometry imaging to investigate lipids in rats
\end{abstract}

Hong Liu, Jianhua Cao, Benjamin Balluff, Audrey C. H. M. Jongen, Marion J. Gijbels, Jarno Melenhorst, Ron M.A. Heeren, and Nicole D. Bouvy Submitted 


\section{Abstract}

\section{Background}

Failure of fascial healing in the abdominal wall can result in incisional hernia, one of the most common complications occurring in $13 \%$ of patients after a laparotomy at two years. Revealing the molecular healing process of abdominal fascia may provide lipid markers of incisional hernia or therapeutic targets that allow prevention or treatment of incisional hernias.

\section{Purpose}

This study aims to investigate temporal and in situ changes of lipids during the normal healing process of abdominal fascia in the first postoperative week.

\section{Methods}

Open hemicolectomy was performed in a total of 35 Wistar rats. The midline fascia was closed identically for all rats using a single continuous suturing technique. These animals were sacrificed with equal numbers $(n=5)$ at each of 7 -time points (6 hours, 12 hours, 24 hours, 48 hours, 72 hours, 120 hours, and 168 hours. The local and temporal changes of lipids were examined with mass spectrometry imaging and correlated to histologically scored changes during healing using hematoxylin and eosin staining.

\section{Results}

Two phosphatidylcholine lipid species (PC O-38:5 and PC 38:4) and one phosphatidylethanolamine lipid PE O-36:5 were found significantly correlated to temporal changes of inflammation. A phosphatidylcholine (PC 32:0) and a monosialodihexosylganglioside (GM3 34:1;2) were found to correlate with fibroblast cell growth.

\section{Conclusion}

Glycerophospholipids and gangliosides are strongly involved in the normal healing process of abdominal fascia and their locally changed concentration might be the potential lipid markers and therapeutic targets of fascial healing. 


\section{Introduction}

Incisional hernia $(\mathrm{IH})$ is one of the most common complications after laparotomy, caused by failed healing of abdominal fascia. ${ }^{1-3}$ The incidence of $\mathrm{IH}$ following laparotomy is around $13 \%$ within two postoperative years ${ }^{4}$ and related costs are estimated to billions of Euros for Europe alone. ${ }^{5,6}$ Despite the significant importance of normal fascial healing in preventing $\mathrm{IH}$, only few studies have illustrated the temporal healing process, especially on the molecular level in the early post-surgery period.

Generally, cellular and molecular events proceed sequentially with four stages, haemostasis, inflammation, proliferation, and remodelling. Despite wound healing of virtually all soft tissues similarly passes through these stages, cells and molecules in fascial healing differ from other specific tissues. ${ }^{7}$ Previous studies have illustrated that the healing rate of the abdominal fascial incision varies from skin wound. ${ }^{8-10}$ Controversy exists with regard to the healing rate of between these two injured tissues. Lemonnier et al. have reported that fascial fibroblast in culture has longer cell-doubling times, larger cell volume, and higher glucose requirements, comparing to dermis fibroblast. ${ }^{10}$ European Hernia Society guidelines recommend that the use of slowly absorbable sutures for the closure of abdominal fascial incision is optimal instead of rapidly absorbable sutures because of the high risk of incisional hernia occurrence when using rapidly absorbable sutures. ${ }^{11,12}$ In contrast, the sutures supporting the skin wound can be removed within one to two weeks postsurgery. However, Michael and Dubay have concluded that the abdominal fascial incision regains tensile strength faster than the cutaneous wound in a rat model. ${ }^{8,9}$

Lipids, acting as signalling molecules as well as structural components of cell membranes, play a critical role in cell structure and function. ${ }^{13,14}$ Lipid mediators can affect cellular biology related to wound healing. For example, diacylglycerol is essential for cellular process in wound healing. ${ }^{15}$ Lysophosphatidic acid enhances the reepithelialisation in the early healing stages of the ear skin in a mouse model. ${ }^{16}$ Arachidonic acid promotes skin wound healing in in-vitro experiments, ${ }^{17}$ and eicosanoids derived from arachidonic acid may modulate inflammatory reactions during the healing process. ${ }^{18}$

Analysis of lipid profiles during wound healing benefits from recent developments in mass spectrometry. ${ }^{19}$ Matrix-assisted laser desorption/ionization-time of flight (MALDI-TOF) mass spectrometry imaging (MSI) can analyze lipids directly from their native tissues and enables label-free visualization of the lipid spatial distribution. ${ }^{20,21}$ Compared to commonly used methods of lipid analysis, like thin-layer chromatography or high-performance liquid chromatography, MALDI-TOF MSI for lipid analysis is less time-consuming while conserving molecular spatial location. ${ }^{22}$ Furthermore, $^{2}$ 
compared to electrospray ionization, MALDI is more sensitive and less affected by impurities. $^{22}$ A previous study has employed MALDI-TOF MSI to identify lipids in skin wound healing process. $^{23}$

This study aims to investigate lipid temporal changes correlated to cellular changes during the normal fascial healing in the first postoperative week in rats. Rat models are chosen in this study to mimic human healing settings as they are genetic similar to humans and it is ethically impossible to isolate functionally healed fascia from patients.

\section{Methods}

\section{Animal experiment}

A total of 35 adult male Wistar rats $(n=35)$, weighing 250-300g, were obtained from Harlan Company. The animal experiment was approved by the Dutch Central Committee (2014-120), complying with the Dutch Animal Experimental Act. Two animals were housed in one Macro cage (EU type IV, $1800 \mathrm{~cm}^{2}$ ) filled with hygiene animal bedding with enrichments of cardboard and wooden block, fitted with 12-hour light-night circle and $25^{\circ} \mathrm{C}$ temperature. Animals could access food $(10 \mathrm{~mm}$ Sniff rat/mouse sterilized food compressed into pellets) and drink (acidified water) ad libitum. Surgeries were performed in a standard operation room in a randomized manner started from the morning of each operation day. All animals were healthy and did not receive any medication treatments before the experiment. After more than one week acclimatization, animals were anesthetized with $2 \%$ isoflurane (IsoFlo ${ }^{\circledR}$, zoeris) and disinfected with $2 \%$ iodine solution. Buprenorphine $0.05 \mathrm{mg} / \mathrm{kg}$ was administered to all animals prior to operations. A midline incision around $5 \mathrm{~cm}$ was made on the abdominal skin and then separately a $5 \mathrm{~cm}$ midline incision on the fascia until the peritoneal cavity. After the ascending colon resection and anastomosis, the fascia was closed continuously with Vicryl TM 4-0 suture (Ethicon Inc; Johnson\&Johnson, Somerville, NJ). Buprenorphine $0.05 \mathrm{mg} / \mathrm{kg}$ was applied postoperatively to all animals when necessary and all animals were checked daily for animal welfare. At each of seven time point ( 6 hours, 12 hours, 24 hours, 48 hours, 72 hours, 120 hours, and 168 hours), five animals $(n=5)$ were sacrificed by cardiac puncture with complete blood drawing under anaesthesia. All animals received the identical surgical procedure. Samples from rats, including fascial incision, sutures and surrounded muscles, were immediately snap frozen with liquid nitrogen and stored in -80 degrees until measurement. 


\section{Sample preparation}

A cryo-microtome (Leica CM 1860 UV, Leica Biosystems, Wetzlar, GE) was preequilibrated to -20 degrees more than half an hour before cutting. Samples were sectioned at $10 \mu \mathrm{m}$ thickness and thaw mounted on pre-cooled Indium Tin Oxide coated (ITO) slide. Slides were dried in desiccator for 15 minutes before matrix application.

Twelve layers of $7 \mathrm{mg} / \mathrm{ml}$ norharmane matrix solution (2:1 chloroform/methanol (v: v)) was applied to the tissue sections using HTX TM-sprayer (HTX Technologies, Chapel Hill, NC) at $30^{\circ} \mathrm{C}$ nozzle temperature and $0.120 \mathrm{ml} / \mathrm{min}$ flow rate. Norharmane and chloroform were obtained from Sigma Aldrich (Zwijndrecht, the Netherlands). Methanol was obtained from Biosolve BV (Valkenswaard, the Netherlands).

\section{MSI data acquisition}

All sections were measured with Bruker Rapiflex MALDI-TOF/TOF Tissuetyper (Bruker Daltonik GmbH, Bremen, Germany) in reflector mode in both ionization modes with mass range of $\mathrm{m} / \mathrm{z} 400-2000$ at pixel size of $50 \mu \mathrm{m} \times 50 \mu \mathrm{m}$. First, tissue sections were analysed at $20 \mu \mathrm{m} \times 20 \mu \mathrm{m}$ scan range (raster size $50 \mu \mathrm{m} \times 50 \mu \mathrm{m}$ ) in the negative ionization mode without offset, and subsequently, measured in the positive ionization mode with an offset of $20 \mu \mathrm{m} \times 20 \mu \mathrm{m}$. Red phosphorus spots were pipetted on each individual slide to calibrate the instrument. Calibration was conducted prior to each analysis.

Two consecutive sections from a 120 hours sample with sufficient different types of cells were chosen to perform high spatial resolution $(10 \mu \mathrm{m})$ mass spectrometry imaging experiments in both ionization modes separately using the same matrix application and MSI instrument as mentioned above. The scan range was $8 \mu \mathrm{m} \times 8 \mu \mathrm{m}$.

MALDI-MS/MS lipid identification was performed_using a Q-Exactive Hybrid Quadrupole-Orbitrap Mass Spectrometer (Thermo Fisher Scientific, Bremen, Germany) coupled to a MALDI source (Spectro-glyph, Kennewick, USA). MS1 spectra in both ionization modes were acquired at mass range $\mathrm{m} / \mathrm{z}$ 400-2000 with 550 milliseconds of injection time. MS2 data of seven masses were performed at 120,000 mass resolution while moving the stage continuously, with averaged 30 scans for each precursor. The normalized collision energy ranged from 20 to $30 \mathrm{eV}$ and isolation window was $\pm 0.7 \mathrm{Da}$ ). The matching of MS1 values and MS2 fragments (when available) for lipid assignments was conducted with the online ALEX123 database (http://alex123.info/ALEX123/MS.php), using a mass tolerance of $3 \mathrm{ppm}$ and 5 ppm, respectively. 


\section{Histological staining after MSI}

All measured tissue sections were stained with haematoxylin and eosin (H\&E) and mounted with coverslips. After air-drying overnight, digital H\&E image was recorded using a Mirax digital slide scanner (Zeiss, Jana, Germany).The inflammatory score and fibroblast score were evaluated blindly determined by an experienced pathologist of Maastricht University (M.G.). The inflammation and fibroblast scores assessed microscopically were described using a $0-5$ scale scores ( 0 , not present; 1 , slightly present; 2 , more than slightly present; 3 , moderately present; 4 , more than moderately present; 5, abundantly present). The inflammation scores refer to the total amount of granulocytes and macrophages. The histological scores were tested with linear regression or generalized linear model, when applicable. Statistical analysis of histological scores was conducted using IBM SPSS Statistics for Windows (version 25.0, Armonk, NY, USA, IBM Corp.).

\section{MSI data processing}

Recalibrations for both the negative and positive ionization mode datasets were performed using FlexAnalysis v3.4 (Bruker Daltonik GmbH, Bremen, Germany) in linear correction mode using $\mathrm{m} / \mathrm{z} 885.6$ and $\mathrm{m} / \mathrm{z} 782.6$ as calibrant, respectively. H\&E images were co-registered with the MSI data using FlexImaging 4.1 software (Bruker Daltonik GmbH, Bremen, Germany), and connective tissues in the fascial incision and adjacent partial muscles were annotated with histological structure. After recalibration, co-registration, and annotation, all data were imported into the SCiLS Lab 2019b (SCiLS GmbH, Bremen, Germany) and normalized to root mean square value.

The average spectra from each dataset was exported to mMass 5.5.0 for peak-picking with the following parameters: (1) signal-to-noise $(\mathrm{S} / \mathrm{N})$ ration was 7.0 , peak-picking height was 75 and 90 for positive and negative ionization mode, respectively (2) baseline correction precision was 35 for both positive and negative ionization mode with relative offset at 0; (3) Deisotoping: maximum charge was 1, isotope mass tolerance was $0.1 \mathrm{~m} / \mathrm{z}$, isotope intensity tolerance was $70 \%$, and isotope mass shift was 0.0. After peak picking, Principal Component Analysis (PCA) with a 95\% confidence ellipse was used to identify and remove outliers in both ionization modes. Linear regression analysis was done in $\mathrm{R}$ (v3.5) to model the relationship between the intensity of any $\mathrm{m} / \mathrm{z}$ species and the healing time. P-values were corrected for multiple testing using the Benjamini-Hochberg method.

Pearson correlation analysis (threshold $\geq 0.4$ ) among different annotated cell regions (typical clusters of smooth muscle cells, granulocytes and macrophages, and fibroblasts) in $10 \mu \mathrm{m}$ high spatial resolution MSI datasets were conducted using the 
significant masses we found in the linear regression analysis to obtain $\mathrm{m} / \mathrm{z}$ species colocalised to inflammation and fibroblast areas.

\section{Results}

\section{Animal experiment}

No complications, including dehiscence of the fascial incision and skin wound, anastomotic leakage, and wound infection, were identified after surgery in 34 out of 35 rats. One rat from the 120 -hour group developed ileus that can affect normal fascial healing negatively because of increased abdominal pressure and then was excluded for further MSI and histological analysis.

\section{Linear regression analysis for $50 \mu \mathrm{m}$ spatial resolution MSI data}

Representative MSI and H\&E images are displayed in Figure 5.1. Five different sections were identified as outliers based on PCA analysis and excluded for further MSI data analysis, including one sample from the 168-hour group in negative ionization mode and four samples (two from the 48-hour group, one from the 72-hour group, and one from the 168-hour group) in positive ionization mode. On average, 174 and $176 \mathrm{~m} / \mathrm{z}$ species were detected after peak picking in negative and positive ionization modes, respectively. A total of 18 and $35 \mathrm{~m} / \mathrm{z}$ species were identified with a significant difference using linear regression analysis in negative and positive ionization modes, respectively (Supplementary Figure S5.1). 


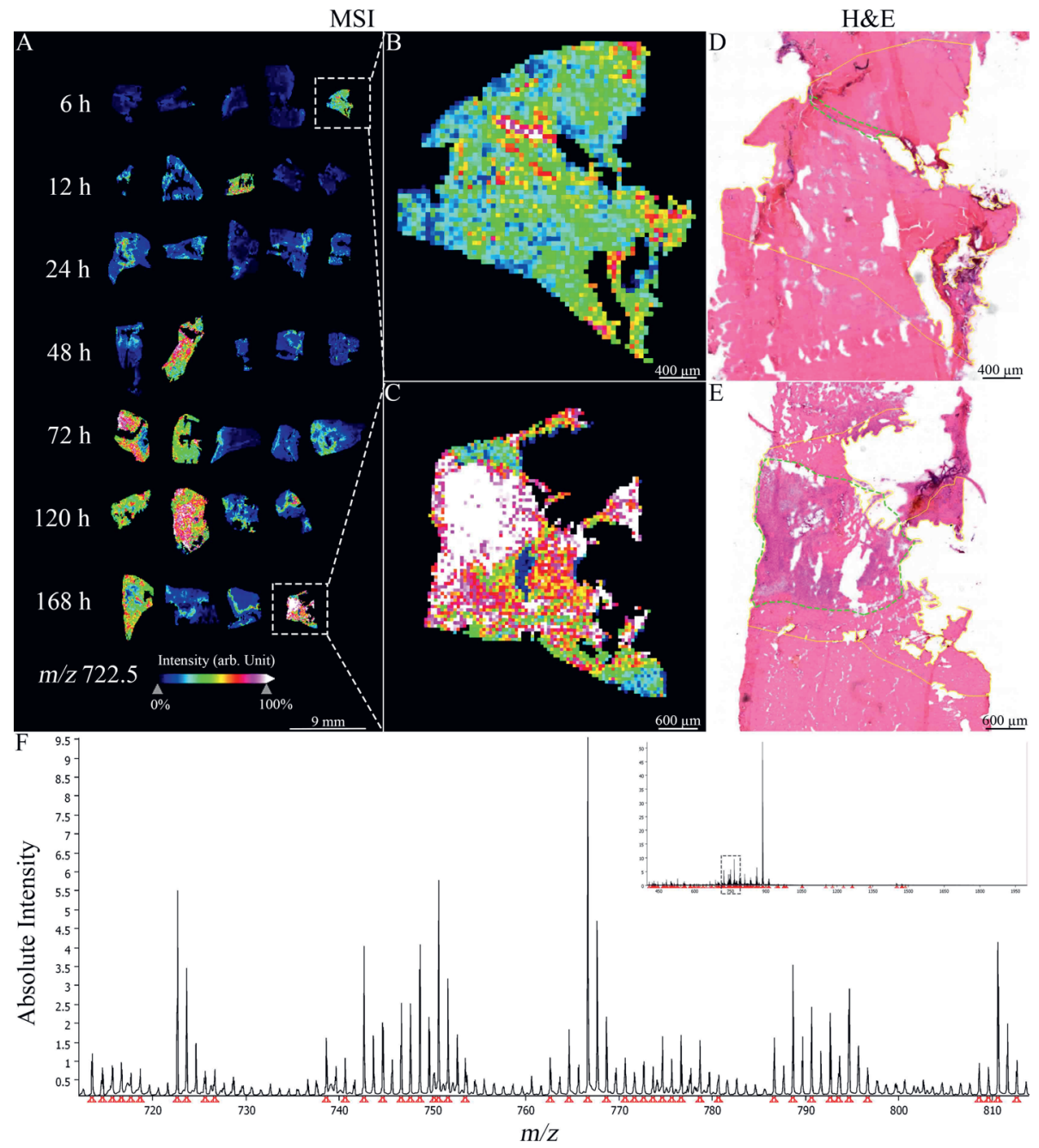

Figure 5.1 Representative MSI and H\&E images. Visualization of $m / z 722.5$ (PE 0-36:5) at different time points in negative ionization mode (A). Magnifications of two representative tissue sections from 6-hour (B) and 168-hour group (C).Their co-registered, hematoxylin and eosin stained ( $H \& E$ ) images in yellow lines ( $D$ and $E$ ). Regions of fascial incision in both H\&E images are indicated with green dashed lines. Average spectra in negative ion mode and magnification at mass range $m / z$ 700-810 (F).

\section{Statistical analysis of histological score data}

The inflammation scores and the fibroblast numbers increased significantly over time using linear regression (slope, 0.017, $P<0.001$ ) and generalized linear model $(P<0.001)$, respectively (Figure 5.2). 
A

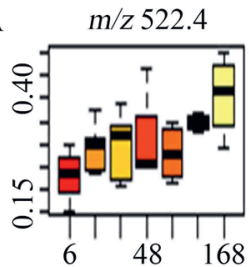

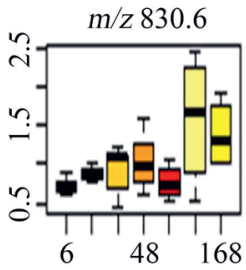
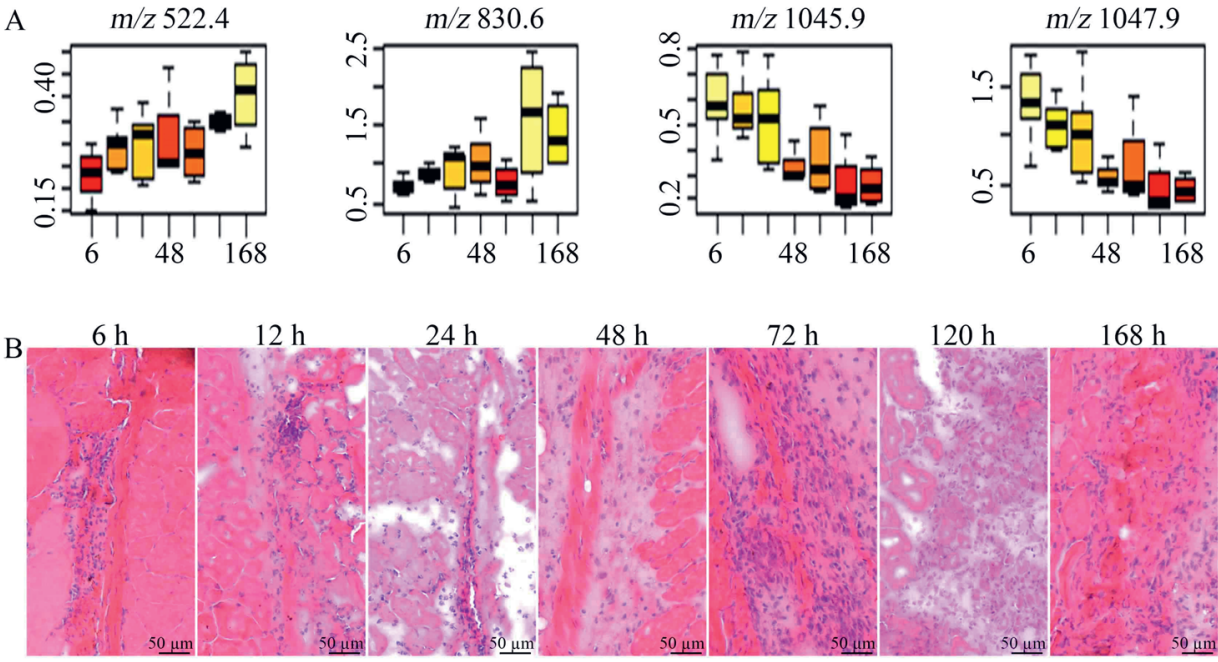

C
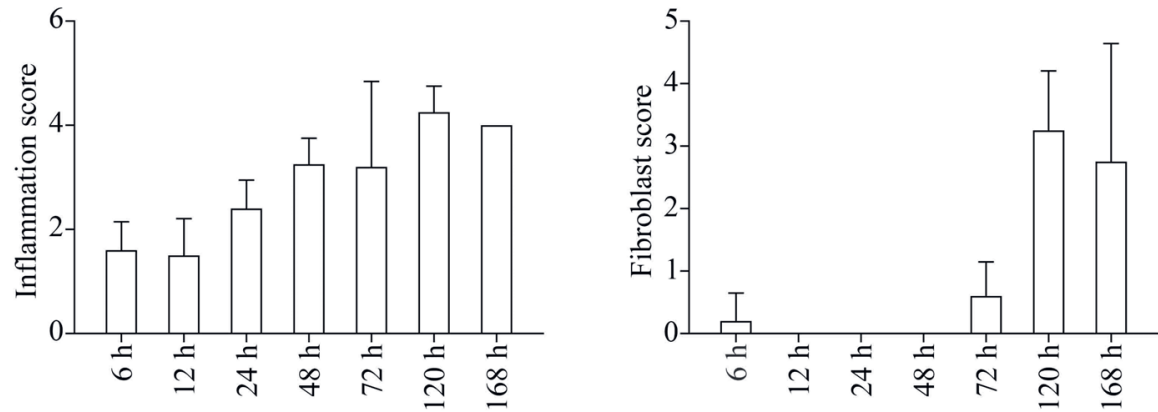

Figure 5.2 A, representative $\mathrm{m} / \mathrm{z}$ values identified with a temporal increase and decline $\mathbf{B}$, representative H\&E images at every time point. $\mathbf{C}$, inflammation and fibroblast scores at each time point. Bar plot, mean \pm standard deviation

\section{Correlation coefficient analysis based on $10 \mu \mathrm{m}$ MSI data}

A total of eight out of $53 \mathrm{~m} / \mathrm{z}$ species were found to correlate with inflammation scores or fibroblast cells with a correlation coefficient of at least 0.4 . All of those eight $\mathrm{m} / \mathrm{z}$ signals, except $\mathrm{m} / \mathrm{z}$ 838.7, were identified and belonged to five lipids (Figure 5.3 and Supplementary Figure S5.2). Tables 5.1 and 5.2 show the lipid identifications in detail. All MS/MS spectra are shown in Supplementary Figures S5.3-S5.9. Two PC lipid species (PC O-38:5 and PC 38:4) and a PE lipid (PE O-36:5) were correlated with inflammation scores. PC 32:0 and GM3 34:1; 2 were found to correlate with fibroblast cells. Representative MSI images of PE O-36:5 and PC O-38:5 in negative and positive ionization mode are shown in Figure 5.3. 
Table 5.1 Four $m / z$ species were correlated to temporal changes of the inflammation.

\begin{tabular}{|c|c|c|c|c|c|c|}
\hline $\begin{array}{l}\text { Observed } \\
\mathrm{m} / \mathrm{z} \text { by TOF }\end{array}$ & $\begin{array}{c}\text { Pearson } \\
\text { correlation } \\
\text { coefficient }\end{array}$ & $\begin{array}{c}\text { Observed m/z } \\
\text { by Orbitrap }\end{array}$ & Lipid assignment & $\begin{array}{l}\text { MS experiment } \\
\text { level for ID }\end{array}$ & $\begin{array}{l}\text { Ion } \\
\text { mode }\end{array}$ & $\begin{array}{l}\text { Mass error } \\
\text { (in ppm) }\end{array}$ \\
\hline 722.5 & 0.57 & 722.5146 & [PE O-36:5-H] & MS2 & NEG & +2.2 \\
\hline 794.7 & 0.66 & 794.6037 & {$[\mathrm{PC} \mathrm{O}-38: 5+\mathrm{H}]$} & MS2 & POS & -2.7 \\
\hline 832.6 & 0.46 & 832.5811 & [PC 38:4+Na] & MS2 & POS & -1.9 \\
\hline 838.7 & 0.46 & 1 & 1 & 1 & POS & / \\
\hline
\end{tabular}

Table $\mathbf{5 . 2}$ Four $m / z$ species were correlated to temporal changes of the fibroblasts.

\begin{tabular}{|c|c|c|c|c|c|c|}
\hline $\begin{array}{l}\text { Observed } \\
\mathrm{m} / \mathrm{z} \text { by TOF }\end{array}$ & $\begin{array}{c}\text { Pearson } \\
\text { correlation } \\
\text { coefficient }\end{array}$ & $\begin{array}{c}\text { Observed m/z } \\
\text { by Orbitrap }\end{array}$ & Lipid assignment & $\begin{array}{c}\text { MS } \\
\text { experiment } \\
\text { level for ID }\end{array}$ & $\begin{array}{l}\text { Ion } \\
\text { mode }\end{array}$ & $\begin{array}{l}\text { Mass error } \\
\text { (in ppm) }\end{array}$ \\
\hline 734.6 & 0.80 & 734.5685 & {$[\mathrm{PC} \mathrm{32:0+H]}$} & MS2 & POS & -1.3 \\
\hline 756.6 & 0.81 & 756.5505 & {$[\mathrm{PC} 32: 0+\mathrm{Na}]$} & MS2 & POS & -1.2 \\
\hline 772.6 & 0.75 & 772.5236 & {$[\mathrm{PC} 32: 0+\mathrm{K}]^{+}$} & MS2 & POS & -2.2 \\
\hline 1151.7 & 0.45 & 1151.7059 & [GM3 34:1;2-H] & MS2 & NEG & +0.0 \\
\hline
\end{tabular}
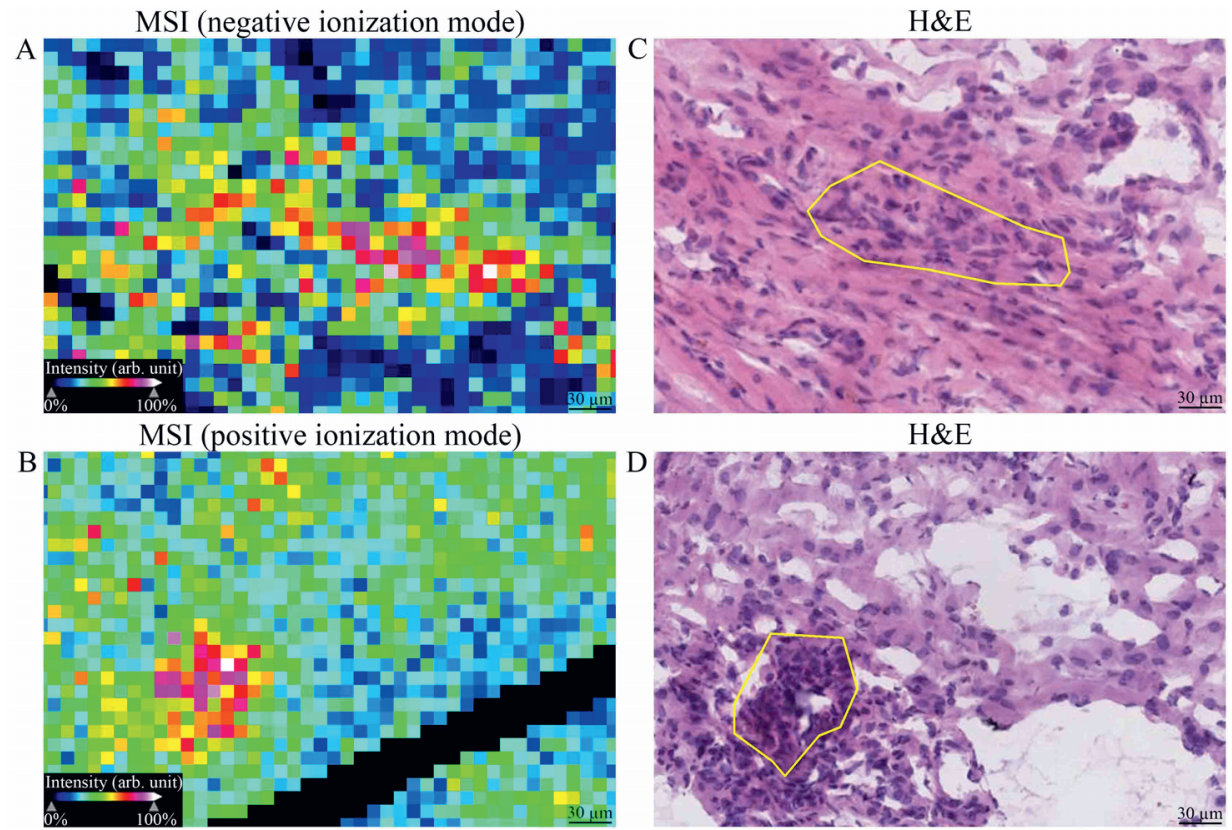

Figure 5.3 Representative MSI images of [PE O-36:5-H]- (A) and [PC 0-38:5+H]+ (B) at $10 \mu \mathrm{m}^{*} 10 \mu \mathrm{m}$ spatial resolution in negative and positive ionization mode, respectively. A magnification of these tissue sections and its co-registered, hematoxylin and eosin stained (H\&E) image are shown in the right side (C and D). Representative inflammation and fibroblast cluster regions are indicated in yellow. 


\section{Discussion}

Molecular changes in abdominal fascia caused by surgical injury are essential for fascial regeneration. ${ }^{24,25}$ Due to very limited comparability within previously published animal models mimicking incisional hernia in patients, ${ }^{26}$ revealing the molecular changes in normal fascial healing over time could provide a better understanding of IH. This study illustrates the temporal changes of lipids correlated to inflammatory response and fibroblast growth in acutely injured fascia. These lipids are potential to be lipid markers of $\mathrm{IH}$ in further animal $\mathrm{IH}$ models or therapeutic targets to promote fascial healing and prevent $\mathrm{IH}$.

Virtually all wound healing pass through similar healing stages. ${ }^{27}$ In skin wound healing, the inflammatory response begins within a few hours after acutely injured, and the proliferative stage occurs from 4 days to 21 days. ${ }^{28}$ In our study, focused on fascial healing, the inflammation score increased overtime and fibroblasts started to increase at the middle of the first postoperative week. This indicates that the healing stages of the inflammation and proliferation in the early fascial healing are comparable to skin wound healing stages. However, abdominal fascial incision heals separately from the adjacent skin wound. ${ }^{8}$ The underlying molecules activated during inflammation and proliferation stages during fascial healing should be different from skin wound healing due to belonging to specific tissues.

The important role of lipids in initiating an acute inflammatory response is widely appreciated. $^{29}$ PCs are one of the most abundant biological components in cell membrane, belonging to a class of phospholipids with choline as a head group. ${ }^{30,31} \mathrm{PE}$ can be converted to PC through PE methyltransferases and involved in the biosynthesis of PC. ${ }^{32,33}$ In our study, two PC lipid species (PC O-38:5 and PC 38:4) and PE O-36:5 were linked to the inflammation score during the normal fascial healing. These lipids found in our study may play an important role in the inflammatory response in fascial healing. The inflammatory response is essential signal to progress into the fibroproliferative stage. ${ }^{34}$ Therefore, these lipids could be inflammatory biomarkers studying $\mathrm{IH}$ in prospective animal models. Furthermore, previous evidence has shown that exogenous PCs can promote mucosal healing in ulcerative colitis in patients ${ }^{35,36}$ and may promote surgical wound healing. ${ }^{37}$ PCs can be isolated from natural sources or obtained using synthetic or semi-sythetic methods and have excellent biocompatibility. ${ }^{38}$ PCs correlated to the inflammatory response in our findings could be applied directly to promote fascial healing in the early postoperative period but further studies are needed.

Fibroblasts contribute significantly in wound healing, creating extracellular matrix components and contracting wound. $^{39}$ Lipid signalling can affect fibroblast 
proliferation. $^{40,41}$ In our study, PC 32:0 and GM3 34:1;2 were linked to the temporal changes of the fibroblasts, indicating that these two lipids could be fibroblast biomarkers in fascial healing. Additionally, a study has demonstrated that proliferating fibroblasts prefer exogenous lipids as the source of membrane lipids rather than de novo synthesis. ${ }^{42}$ GM3 analogues can be synthesized conveniently from commercially available substances. ${ }^{43}$ Application of PC 32:0 or GM3 34:1;2 as potential agents could be a novel strategy promoting fibroblast growth in fascial incision despite further researches needed. It should be noted that GM3 belonging to gangliosides is involved in mediating grow factor signalling in human skins. GM3 is observed to be a driver of impaired wound healing and depletion of GM3 can result in improved wound healing in diabetic mice. ${ }^{44,45}$ However, in our studies, GM3 34:1;2 increased over time, positively linked to the increase of the fibroblasts. This could be explained by that tissue-specific involved-molecules differ between fascial healing and skin wound healing.

Animal models have intrinsic limitations. However, a previous study has demonstrated the molecular comparability between rats and humans using gene expression comparison, regarding healing and scarring. ${ }^{46}$ Furthermore, regarding an IH model, histological results between rats and humans are comparable. ${ }^{47}$ Therefore, the present results could be translated to humans despite further in vivo human confirmation is necessary.

\section{Conclusion}

In summary, five lipids, including PE O-36:5, PC O-38:5, PC 38:4, PC 32:0, and GM3 $34: 1 ; 2$, were found significantly involved in the fascial healing and might be potential lipid markers and therapeutic targets preventing IH. Further studies evaluating the functional effect of these lipids in fascial healing are needed. 


\section{References}

1. Kohler A, Lavanchy JL, Lenoir U, Kurmann A, Candinas D, Beldi G. Effectiveness of Prophylactic Intraperitoneal Mesh Implantation for Prevention of Incisional Hernia in Patients Undergoing Open Abdominal Surgery: A Randomized Clinical Trial. JAMA Surg. 2019;154(2):109-15.

2. Jairam AP, Timmermans L, Eker HH, Pierik R, van Klaveren D, Steyerberg EW, et al. Prevention of incisional hernia with prophylactic onlay and sublay mesh reinforcement versus primary suture only in midline laparotomies (PRIMA): 2-year follow-up of a multicentre, double-blind, randomised controlled trial. Lancet. 2017;390(10094):567-76.

3. Xing L, Culbertson EJ, Wen Y, Franz MG. Early laparotomy wound failure as the mechanism for incisional hernia formation. J Surg Res. 2013;182(1):e35-42.

4. Bosanquet DC, Ansell J, Abdelrahman T, Cornish J, Harries R, Stimpson A, et al. Systematic Review and Meta-Regression of Factors Affecting Midline Incisional Hernia Rates: Analysis of 14,618 Patients. PLoS One. 2015;10(9):e0138745.

5. Gillion JF, Sanders D, Miserez M, Muysoms F. The economic burden of incisional ventral hernia repair: a multicentric cost analysis. Hernia. 2016;20(6):819-30.

6. Poulose BK, Shelton J, Phillips S, Moore D, Nealon W, Penson D, et al. Epidemiology and cost of ventral hernia repair: making the case for hernia research. Hernia. 2012;16(2):179-83.

7. Kumka M, Bonar J. Fascia: a morphological description and classification system based on a literature review. J Can Chiropr Assoc. 2012;56(3):179-91.

8. Franz MG, Smith PD, Wachtel TL, Wright TE, Kuhn MA, Ko F, et al. Fascial incisions heal faster than skin: a new model of abdominal wall repair. Surgery. 2001;129(2):203-8.

9. Dubay DA, Wang X, Kirk S, Adamson B, Robson MC, Franz MG. Fascial fibroblast kinetic activity is increased during abdominal wall repair compared to dermal fibroblasts. Wound Repair Regen. 2004; 12(5):539-45.

10. Lemonnier F, Gautier M, Wolfrom C, Lemonnier A. Some metabolic differences between human skin and aponeurosis fibroblasts in culture. J Cell Physiol. 1980;104(3):415-23.

11. Muysoms FE, Antoniou SA, Bury K, Campanelli G, Conze J, Cuccurullo D, et al. European Hernia Society guidelines on the closure of abdominal wall incisions. Hernia. 2015;19(1):1-24.

12. Diener MK, Voss S, Jensen K, Buchler MW, Seiler CM. Elective midline laparotomy closure: the INLINE systematic review and meta-analysis. Ann Surg. 2010;251(5):843-56.

13. Santos AL, Preta G. Lipids in the cell: organisation regulates function. Cell Mol Life Sci. 2018;75(11):1909-27.

14. Muro E, Atilla-Gokcumen GE, Eggert US. Lipids in cell biology: how can we understand them better? Mol Biol Cell. 2014;25(12):1819-23.

15. Vaughan EM, You JS, Elsie Yu HY, Lasek A, Vitale N, Hornberger TA, et al. Lipid domain-dependent regulation of single-cell wound repair. Mol Biol Cell. 2014;25(12):1867-76.

16. Demoyer JS, Skalak TC, Durieux ME. Lysophosphatidic acid enhances healing of acute cutaneous wounds in the mouse. Wound Repair Regen. 2000;8(6):530-7.

17. Oh SY, Lee SJ, Jung YH, Lee HJ, Han HJ. Arachidonic acid promotes skin wound healing through induction of human MSC migration by MT3-MMP-mediated fibronectin degradation. Cell Death Dis. 2015;6:e1750.

18. Dhall S, Wijesinghe DS, Karim ZA, Castro A, Vemana HP, Khasawneh FT, et al. Arachidonic acidderived signaling lipids and functions in impaired healing. Wound Repair Regen. 2015;23(5):644-56.

19. Wijesinghe DS, Chalfant CE. Systems-Level Lipid Analysis Methodologies for Qualitative and Quantitative Investigation of Lipid Signaling Events During Wound Healing. Adv Wound Care (New Rochelle). 2013;2(9):538-48.

20. Leopold J, Popkova Y, Engel KM, Schiller J. Recent Developments of Useful MALDI Matrices for the Mass Spectrometric Characterization of Lipids. Biomolecules. 2018;8(4).

21. Cerruti CD, Touboul D, Guerineau V, Petit VW, Laprevote O, Brunelle A. MALDI imaging mass spectrometry of lipids by adding lithium salts to the matrix solution. Anal Bioanal Chem. 2011;401(1):75-87. 
22. Schiller J, Suss R, Arnhold J, Fuchs B, Lessig J, Muller M, et al. Matrix-assisted laser desorption and ionization time-of-flight (MALDI-TOF) mass spectrometry in lipid and phospholipid research. Prog Lipid Res. 2004;43(5):449-88.

23. Lewis EEL, Barrett MRT, Freeman-Parry L, Bojar RA, Clench MR. Examination of the skin barrier repair/wound healing process using a living skin equivalent model and matrix-assisted laser desorption-ionization-mass spectrometry imaging. Int J Cosmet Sci. 2018;40(2):148-56.

24. Zugel M, Maganaris CN, Wilke J, Jurkat-Rott K, Klingler W, Wearing SC, et al. Fascial tissue research in sports medicine: from molecules to tissue adaptation, injury and diagnostics: consensus statement. Br J Sports Med. 2018;52(23):1497.

25. Franz MG. The biology of hernia formation. Surg Clin North Am. 2008;88(1):1-15, vii.

26. Vogels RRM, Kaufmann R, van den Hil LCL, van Steensel S, Schreinemacher MHF, Lange JF, et al. Critical overview of all available animal models for abdominal wall hernia research. Hernia. 2017;21(5):667-75.

27. Velnar T, Bailey T, Smrkolj V. The wound healing process: an overview of the cellular and molecular mechanisms. J Int Med Res. 2009;37(5):1528-42.

28. Childs DR, Murthy AS. Overview of Wound Healing and Management. Surg Clin North Am. 2017;97(1):189-207.

29. Serhan CN, Chiang N, Dalli J, Levy BD. Lipid mediators in the resolution of inflammation. Cold Spring Harb Perspect Biol. 2014;7(2):a016311.

30. Kanno K, Wu MK, Scapa EF, Roderick SL, Cohen DE. Structure and function of phosphatidylcholine transfer protein (PC-TP)/StarD2. Biochim Biophys Acta. 2007;1771(6):654-62.

31. Li Z, Vance DE. Phosphatidylcholine and choline homeostasis. J Lipid Res. 2008;49(6):1187-94.

32. Calzada E, Onguka O, Claypool SM. Phosphatidylethanolamine Metabolism in Health and Disease. Int Rev Cell Mol Biol. 2016;321:29-88.

33. Sohlenkamp C, Lopez-Lara IM, Geiger O. Biosynthesis of phosphatidylcholine in bacteria. Prog Lipid Res. 2003;42(2):115-62.

34. Robson MC, Steed DL, Franz MG. Wound healing: biologic features and approaches to maximize healing trajectories. Curr Probl Surg. 2001;38(2):72-140.

35. Antonelli E, Villanacci V, Bassotti G. Novel oral-targeted therapies for mucosal healing in ulcerative colitis. World J Gastroenterol. 2018;24(47):5322-30.

36. Karner M, Kocjan A, Stein J, Schreiber S, von Boyen G, Uebel P, et al. First multicenter study of modified release phosphatidylcholine "LT-02" in ulcerative colitis: a randomized, placebo-controlled trial in mesalazine-refractory courses. Am J Gastroenterol. 2014;109(7):1041-51.

37. Kornilova ZK, Selishcheva AA, Perel'man MI. Effect of phosphatidylcholine liposome on regeneration of surgical wound in guinea pig lung. Bull Exp Biol Med. 2001;131(2):191-4.

38. Li J, Wang XL, Zhang T, Wang CL, Huang ZJ, Luo X, et al. A review on phospholipids and their main applications in drug delivery systems. Asian J Pharm Sci. 2015;10(2):81-98.

39. desJardins-Park HE, Foster DS, Longaker MT. Fibroblasts and wound healing: an update. Regen Med. 2018;13(5):491-5.

40. Qu X, Zhang X, Yao J, Song J, Nikolic-Paterson DJ, Li J. Resolvins E1 and D1 inhibit interstitial fibrosis in the obstructed kidney via inhibition of local fibroblast proliferation. J Pathol. 2012; 228(4):506-19.

41. Mkrtschjan MA, Gaikwad SB, Kappenman KJ, Solis C, Dommaraju S, Le LV, et al. Lipid signaling affects primary fibroblast collective migration and anchorage in response to stiffness and microtopography. J Cell Physiol. 2018;233(4):3672-83.

42. Yao CH, Fowle-Grider R, Mahieu NG, Liu GY, Chen YJ, Wang R, et al. Exogenous Fatty Acids Are the Preferred Source of Membrane Lipids in Proliferating Fibroblasts. Cell Chem Biol. 2016;23(4): 483-93.

43. Zheng C, Huang R, Bavaro T, Terreni M, Sollogoub M, Xu J, et al. Design, synthesis and biological evaluation of new ganglioside GM3 analogues as potential agents for cancer therapy. Eur J Med Chem. 2020;189:112065.

44. Dam DHM, Paller AS. Gangliosides in Diabetic Wound Healing. Prog Mol Biol Transl Sci. 2018; 156:229-39. 
45. Wang XQ, Lee S, Wilson H, Seeger M, Iordanov H, Gatla N, et al. Ganglioside GM3 depletion reverses impaired wound healing in diabetic mice by activating IGF-1 and insulin receptors. J Invest Dermatol. 2014;134(5):1446-55.

46. Occleston NL, Metcalfe AD, Boanas A, Burgoyne NJ, Nield K, O'Kane S, et al. Therapeutic improvement of scarring: mechanisms of scarless and scar-forming healing and approaches to the discovery of new treatments. Dermatol Res Pract. 2010;2010.

47. van den Hil LCL, Vogels RRM, van Barneveld KWY, Gijbels MJJ, Peutz-Kootstra CJ, Cleutjens JPM, et al. Comparability of histological outcomes in rats and humans in a hernia model. J Surg Res. 2018;229:271-6. 


\section{Supplemental material}

A
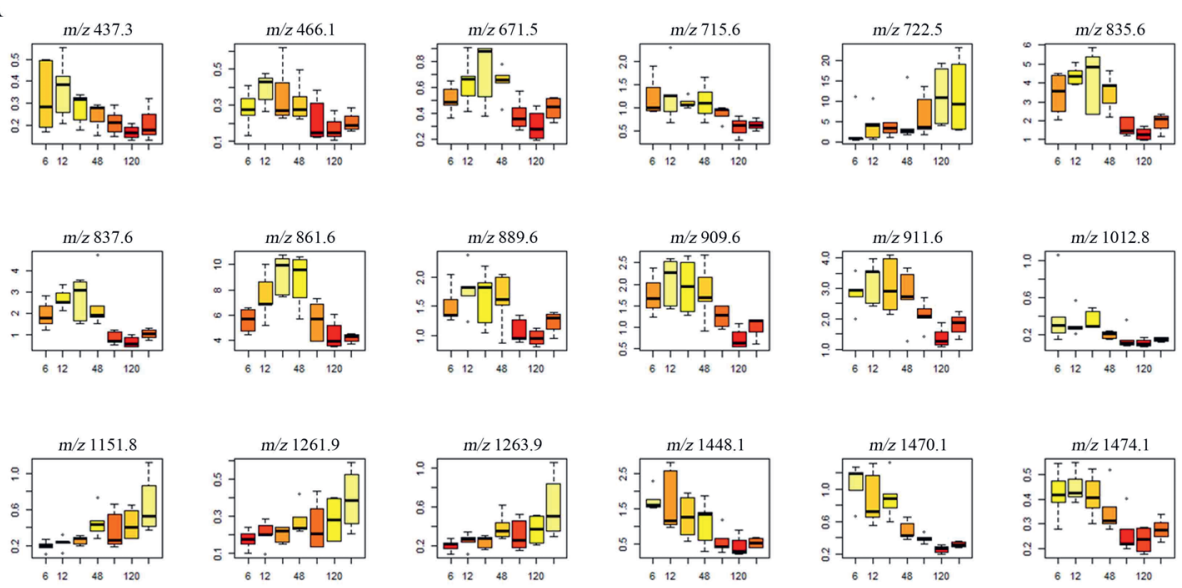

B
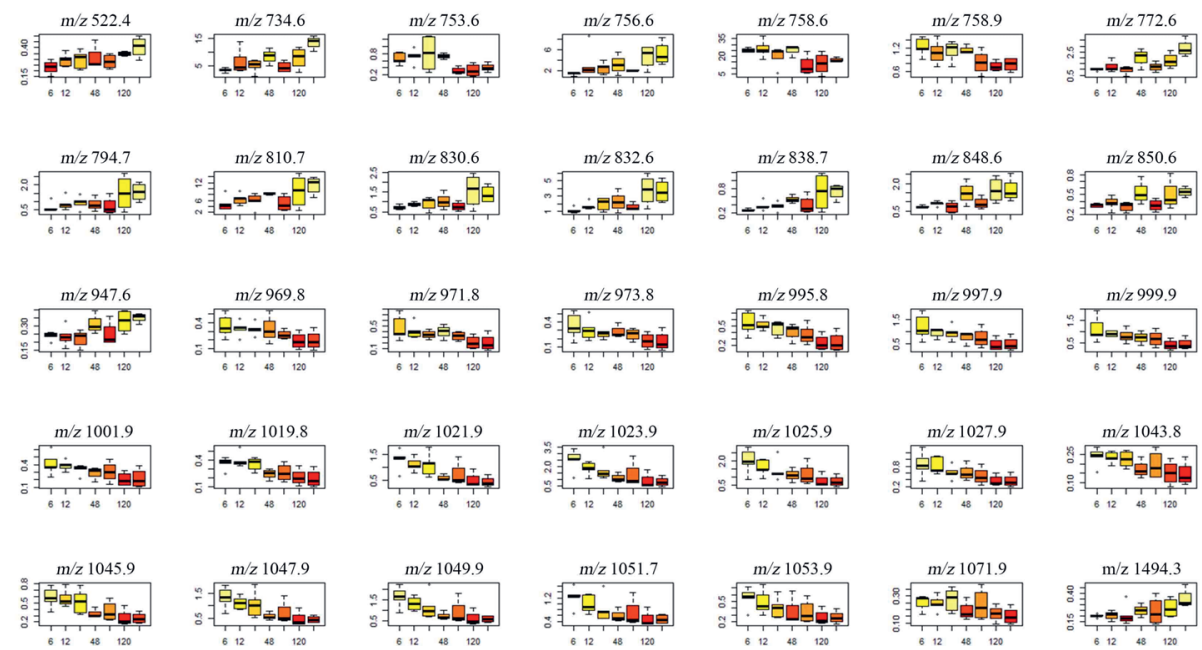

$\frac{m / z \quad 1053.9}{2}$
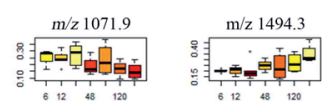

Figure S5.1 Linear regression with significant difference $(P<0.05)$ for $m / z$ species in negative $(A)$ and positive ionization modes (B). 
MSI (negative ionization mode)

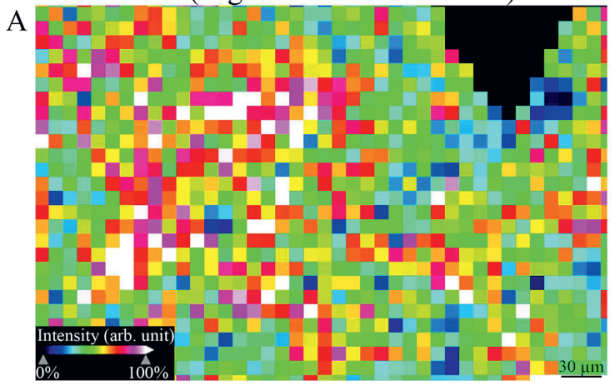

MSI (positive ionization mode)

B

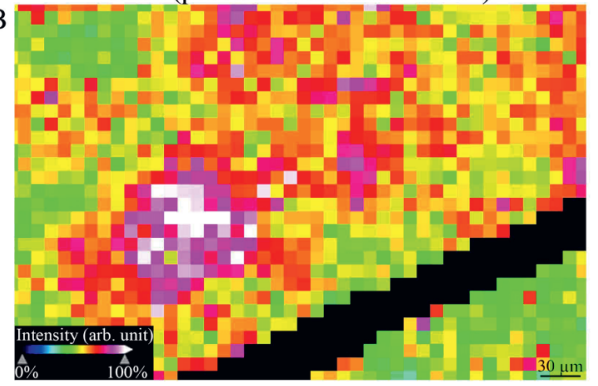

MSI (positive ionization mode)

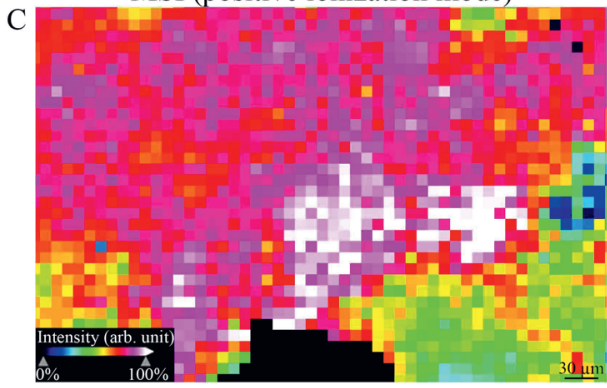

$\mathrm{H} \& \mathrm{E}$

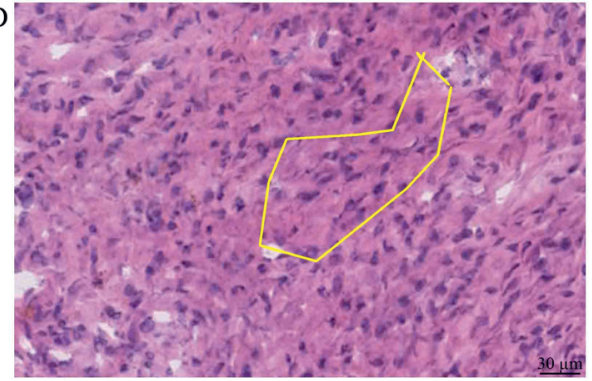

$\mathrm{H} \& \mathrm{E}$

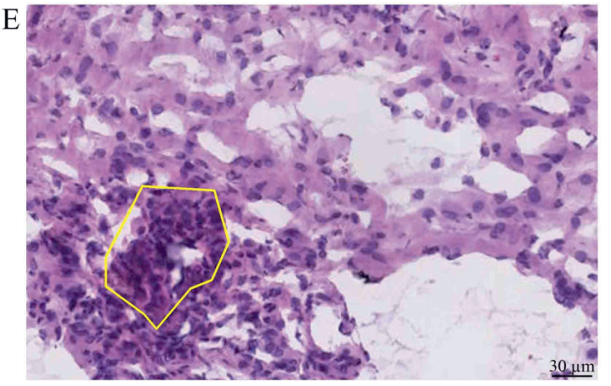

$\mathrm{H} \& \mathrm{E}$

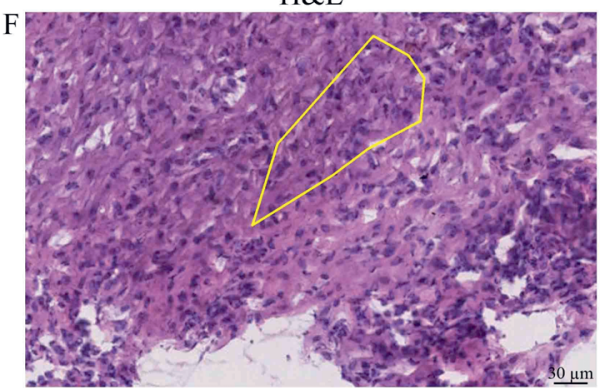

Figure S5.2 Representative MSI images of [GM 34:1;2-H] (A), [PC 38:4+Na] $]^{+}(B)$, and [PC 32:0+H] ${ }^{+}(C)$, at $10 \mu \mathrm{m} * 10 \mu \mathrm{m}$ spatial resolution in negative and positive ionization mode, respectively. A magnification of these tissue sections and its co-registered, hematoxylin and eosin stained (H\&E) image are shown in the right side (D, E, and F). Inflammation and fibroblast cluster regions are indicated in yellow. 


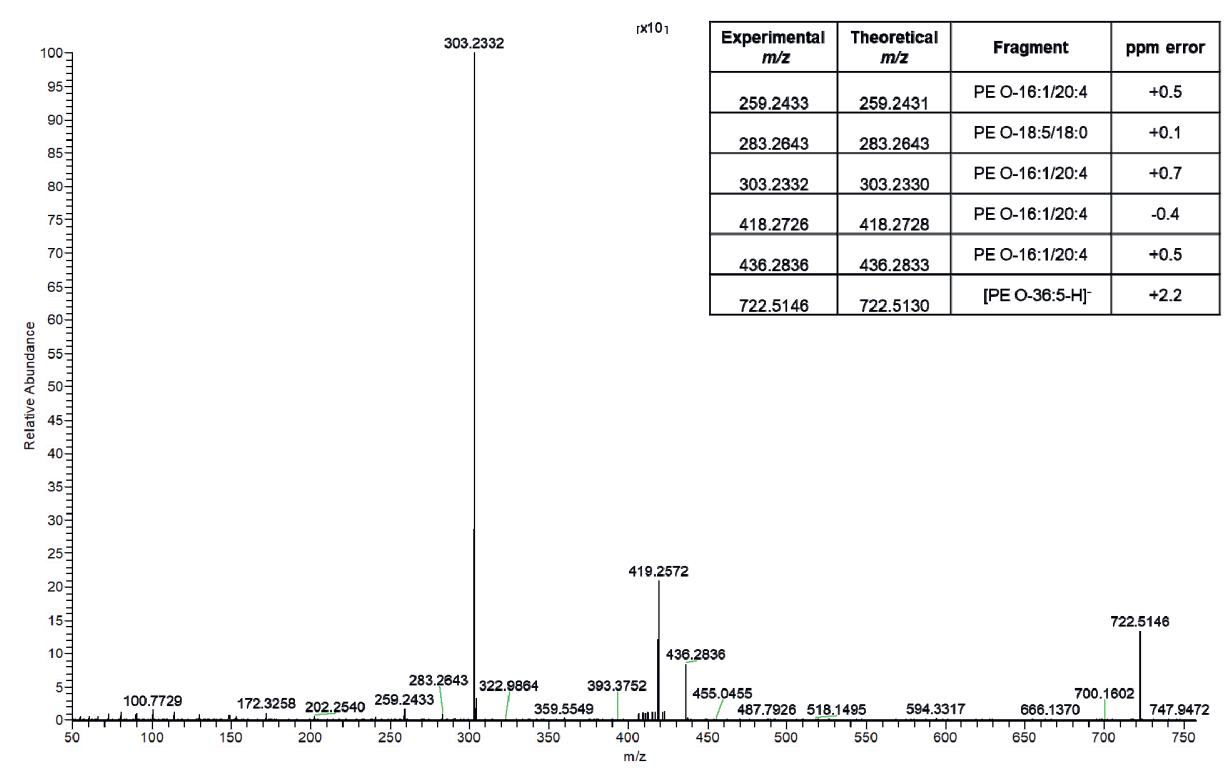

Figure S5.3 MS/MS spectrum of $m / z 722.5146$ ([PE O-36:5-H]).

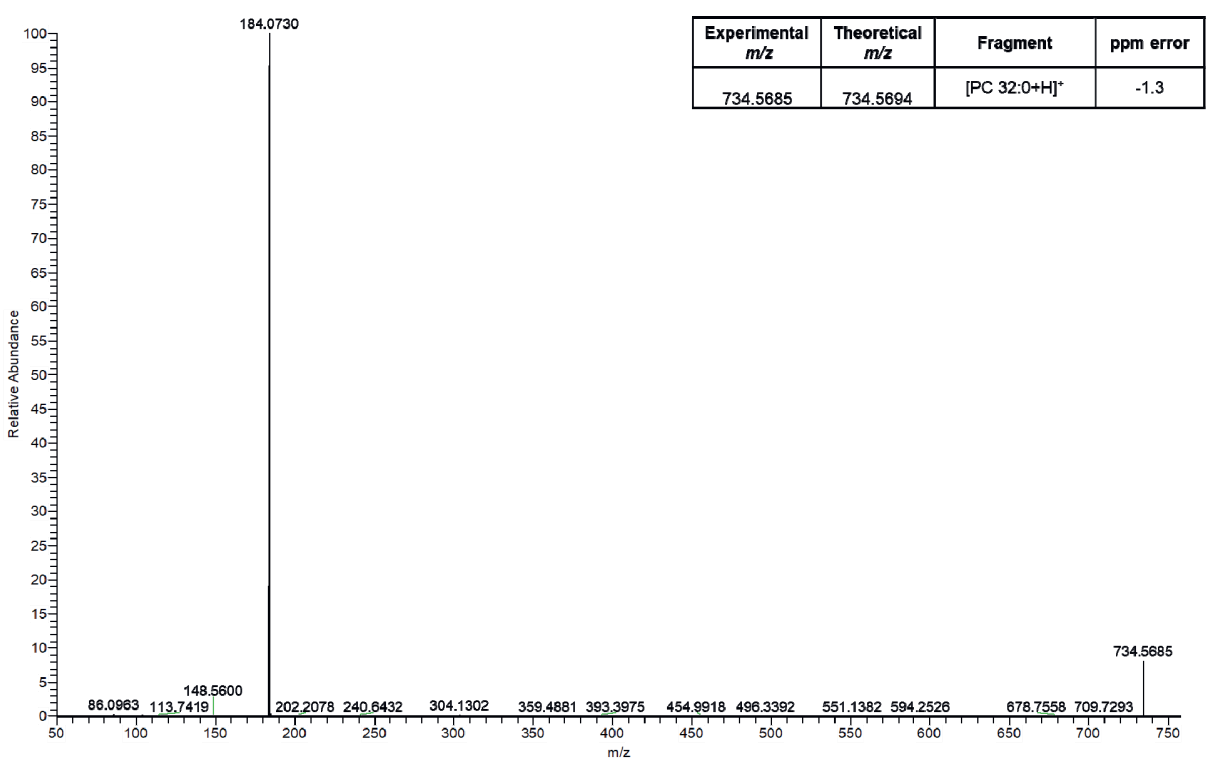

Figure S5.4 MS/MS spectrum of $m / z 734.5685\left([\mathrm{PC} 32: 0+\mathrm{H}]^{+}\right)$. 


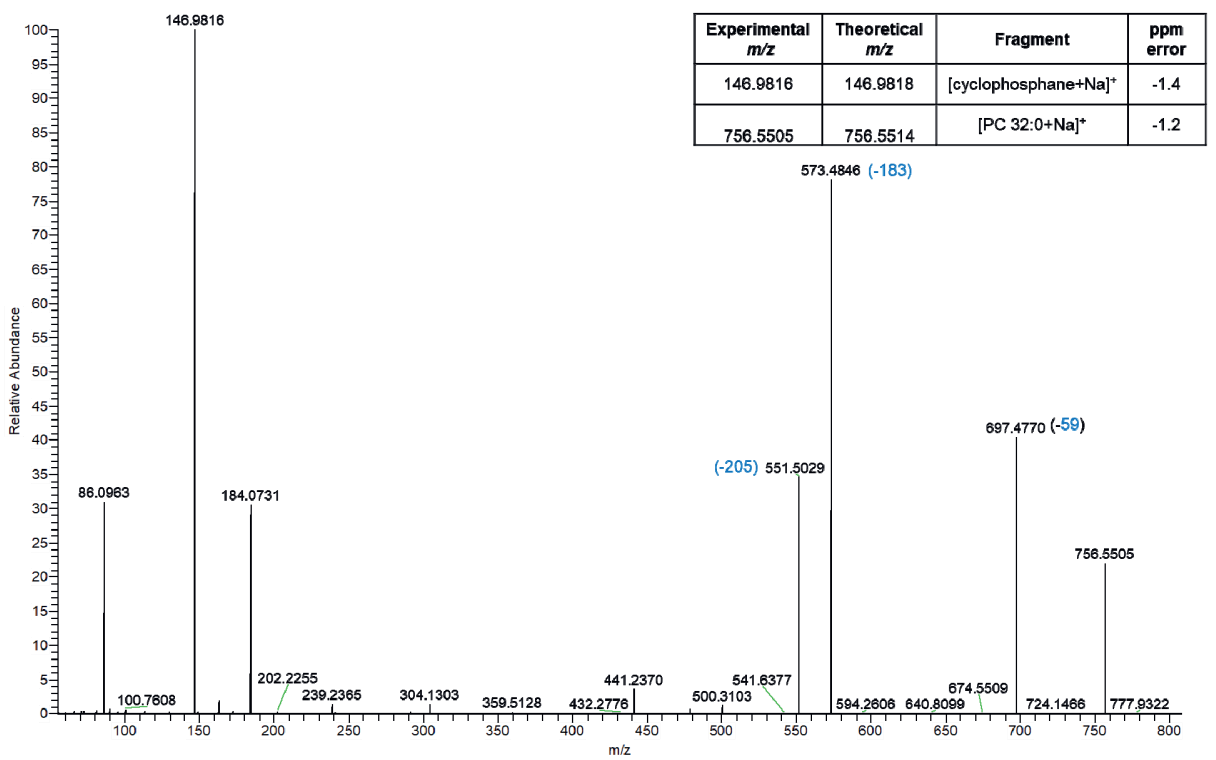

Figure S5.5 MS/MS spectrum of $m / z 756.5505\left([\mathrm{PC} 32: 0+\mathrm{Na}]^{+}\right)$.

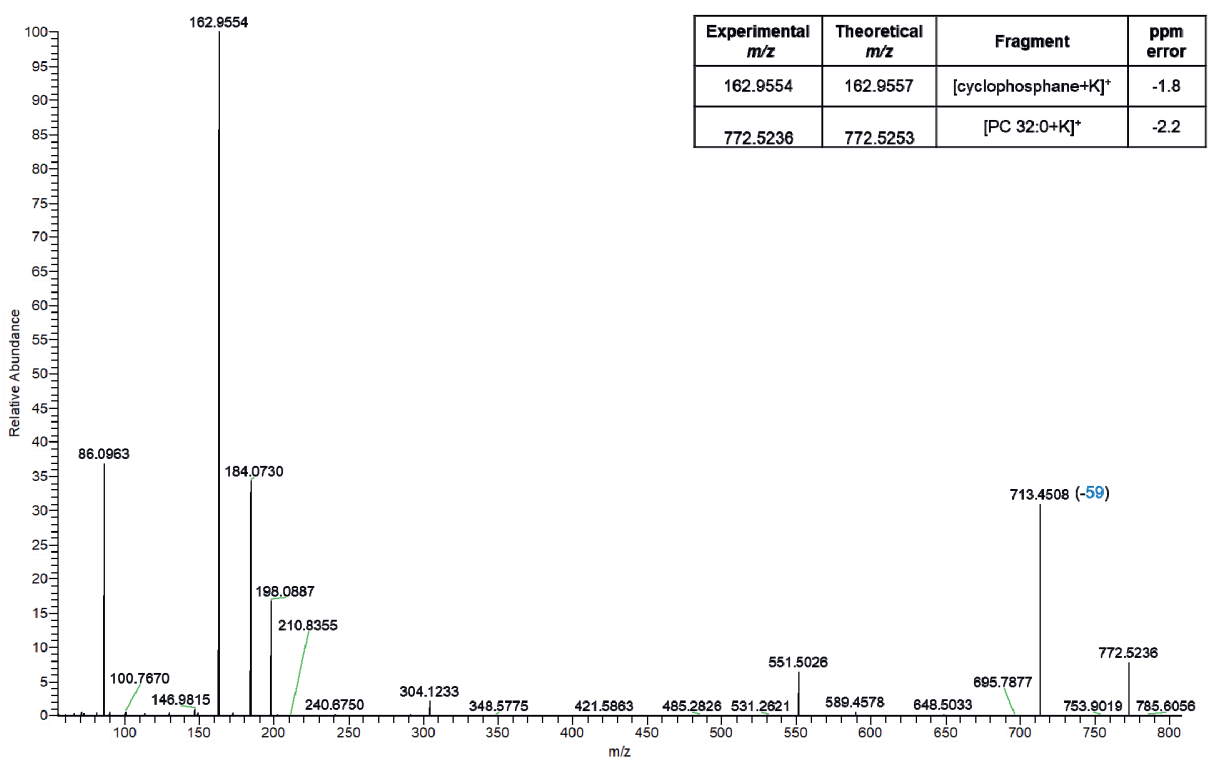

Figure S5.6 MS/MS spectrum of $m / z 772.5236\left([\mathrm{PC} 32: 0+\mathrm{K}]^{+}\right)$. 


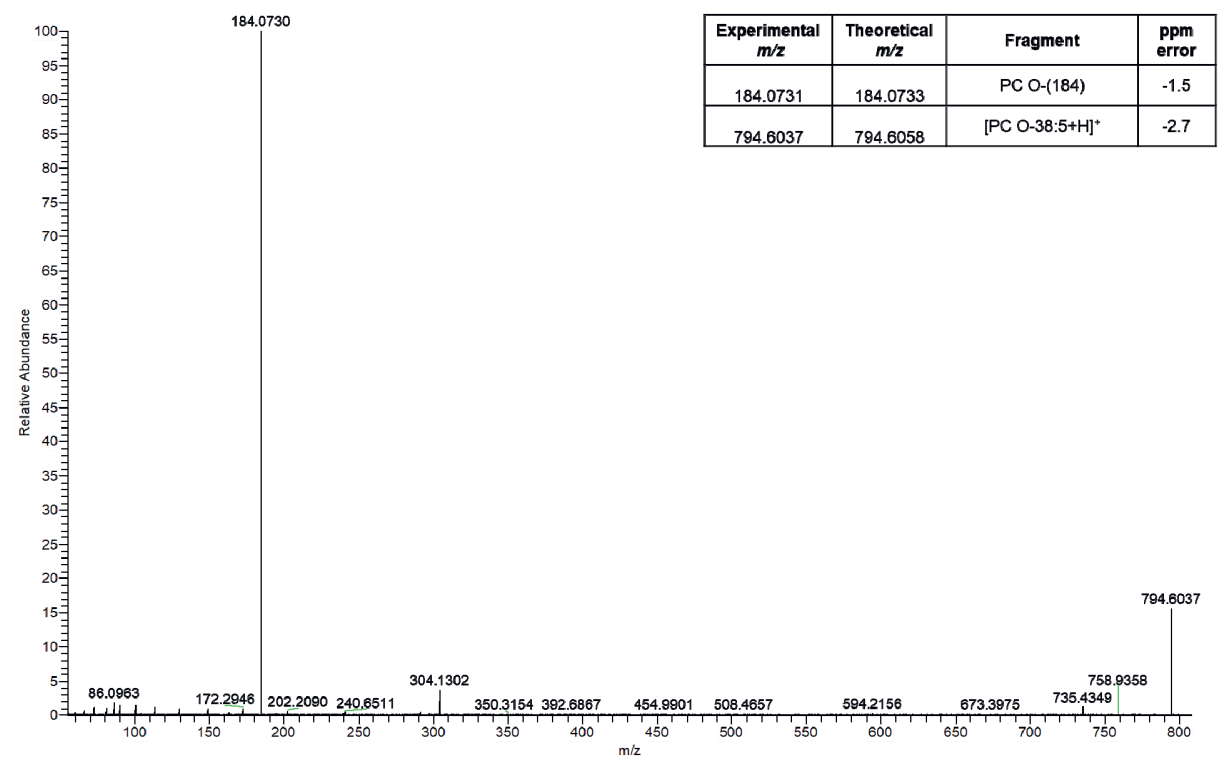

Figure S5.7 MS/MS spectrum of $m / z 794.6037\left([\mathrm{PC} \mathrm{O}-38: 5+\mathrm{H}]^{+}\right)$.

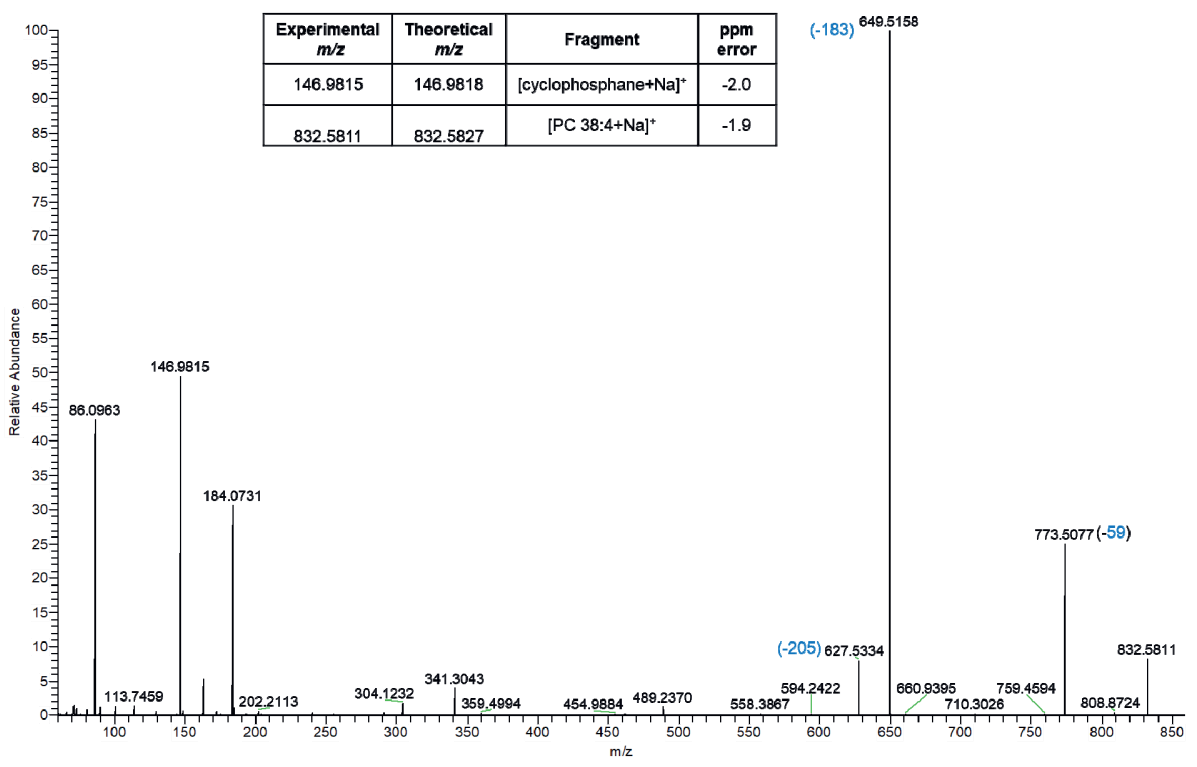

Figure S5.8 MS/MS spectrum of $m / z 832.5811\left([\mathrm{PC} 38: 4+\mathrm{Na}]^{+}\right)$. 


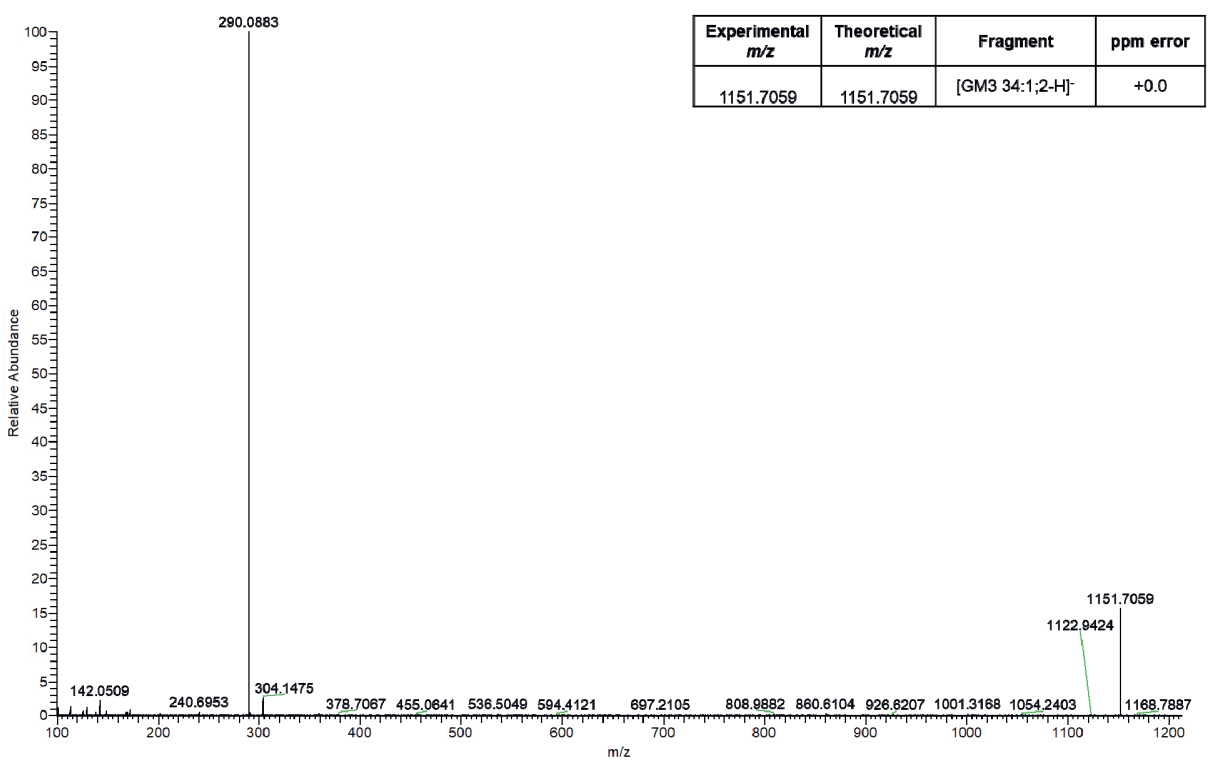

Figure S5.9 MS/MS spectrum of $m / z 1151.7059$ ([GM3 34:1;2-H]'). 

Part III

Too much repaired tissue in case of adhesions 



\section{Chapter 6}

Comparing five new polymer barriers for the
prevention of intra-abdominal adhesions in a
rat model

Sebastiaan van Steensel, Hong Liu, Elwin H.H. Mommers, Kaatje Lenaerts, Nicole D. Bouvy The Journal of Surgical Research 2019;243:453-459 


\section{Abstract}

\section{Background and objective}

Intra-abdominal adhesions affect up to $93 \%$ of the patients after abdominal surgery, causing small-bowel obstruction, infertility, chronic abdominal pain, and iatrogenic bowel injury at reoperation. The efficacy of five new polymer antiadhesive barriers to avoid adhesion formation is evaluated in an ischemic button model in rats.

\section{Materials and methods}

Five new, biodegradable polyurethane and copolyester-based, antiadhesive barriers (A1, A2, A3, B1, and B2) were evaluated in separate experimental groups and compared with two control groups (hyaluronate carboxymethylcellulose barrier and no antiadhesive barrier) in an ischemic button model ( $\mathrm{n}=11$ per group operated). After $14 \mathrm{~d}$, the quantity and quality of the adhesions were scored macroscopically. The Kruskal-Wallis with Mann-Whitney $U$ post hoc and the Fisher's exact tests were used for data analysis. The Bonferroni correction method was applied, and a $P$-value $<0.007$ was considered significant.

\section{Results}

Two animals died during surgery and follow-up. A significant reduction of adhesions to ischemic buttons was found in the A2 group (median, 3.5; interquartile range, 2.25) compared with no adhesive barrier (median, 8.0; interquartile range, 2.0) $(P=0.001)$. The remaining groups did not differ significantly regarding adhesion quantity or quality. Adverse events were observed in the A2, A3, and B2 groups.

\section{Conclusions}

The A2 antiadhesive barrier reduced the adhesion formation significantly compared with no antiadhesive barrier, but applicability is questionable because of extensive adverse events observed due to implantation of the antiadhesive barrier. The Nair score appears not to be sensitive enough to detect differences in adhesion formation in this model. Future research should focus on antiadhesive barriers that are self-adhering. 


\section{Introduction}

Intra-abdominal adhesion formation is a common complication after abdominal surgery with an incidence reported from $79 \%$ to $93 \%{ }^{1,2}$ Adhesions cause $56 \%$ of small-bowel obstructions, cause the need for fertility treatment in $23 \%$ of the female patients, and are the most likely cause of chronic abdominal pain in $57 \%$ of the patients with a history of abdominal surgery. ${ }^{3}$ Furthermore, due to adhesions, re-entry to the abdominal cavity is accompanied by an increased risk of iatrogenic bowel injury., Subsequently, this is related to an increase in the incidence of sepsis, intra-abdominal complications, wound infections, a prolonged hospital stay, and higher costs. ${ }^{4}$

The risk of being readmitted $5 \mathrm{y}$ after abdominal surgery because of morbidity directly related to adhesions is $3.8 \%$, with an average readmission rate of 2.2 per patient. ${ }^{5,6}$ This risk is reduced to $3.0 \%$ when a laparoscopic approach is used. ${ }^{7}$ Still, in $37.7 \%$ of the patients, adhesions are observed after laparoscopic surgery, ${ }^{2}$ leaving preventive measures to avoid adhesion formation still of the utmost importance.

Antiadhesive barriers can be used in the prevention of intra-abdominal adhesions. Oxidized regenerated cellulose (Interceed, Ethicon, US, NJ) showed a reduction of adhesions in gynaecologic patients, but no data regarding the reoperation rate are available. Hyaluronate carboxymethylcellulose (HA-CMC) (Seprafilm, Sanofi, US, NJ) proved to be effective in the reduction of adhesion formation and decreasing the number of reoperations. ${ }^{8}$ HA-CMC reduces adhesion formation in $25.9 \%$ of the cases, ${ }^{9}$ although given the high incidence, further adhesion reduction is desired. These rather unsatisfactory results translate in a low confidence of surgeons toward the use of antiadhesive barriers in clinical practice, as shown in the Dutch National survey among surgeons. ${ }^{10}$

The lack of a sufficiently effective, preventive measure inspires the search for a new antiadhesive barrier. Five polymer sheets of different composition are evaluated in this animal study. The aim is to identify the most effective antiadhesive barrier in an ischemic button model in rats in comparison with no antiadhesive barrier placement and to a currently available antiadhesive barrier.

\section{Materials and methods}

The study protocol (AVD107002016720) was approved by the ethical committee of animal experiments, which complied to the Dutch Animal Experimental Act and the European Directive 2010/63/EU. 


\section{Study design}

The animals were equally divided into seven groups ( $\mathrm{n}=11$ per group), five intervention groups (A1, A2, A3, B1, and B2) and two control groups (HA-CMC barrier and no adhesive barrier). All groups except for one control group received a $5 \mathrm{~cm}$ antiadhesive barrier, either HA-CMC or one of the interventional barriers. The group receiving HA$\mathrm{CMC}$ was used as a negative control, providing information regarding the effect of the interventional antiadhesive barriers compared with what is currently available on the market. A computer generated a random sequence regarding the group allocation of the animals. To blind the surgeons, envelopes containing the unmarked antiadhesive barriers were arranged in that sequence. Because two antiadhesive barriers had a rough surface and a positive control group without an antiadhesive barrier was present, complete blinding of the surgeon could not be achieved. During surgery, consequent envelopes were opened and the animal received the barrier accordingly, and in case the animal was allocated to the control group, the envelope was empty. Throughout the experiment, the allocation of the animals remained unknown, so the adhesion assessment at sacrifice and data analysis was performed blindly. The randomization and blinding process were conducted by a researcher not involved in the experiment. The follow-up was $14 \mathrm{~d}$.

\section{Materials}

Five different antiadhesive barriers (A1, A2, A3, B1, and B2) were evaluated for their antiadhesive properties. Three barriers were based on a linear, segmented biodegradable polyurethane (A1, A2, and A3). A1 and A2 were different in their hard segment composition, and A3 was enriched with bioactive glass particles on one side. Two barriers consisted of high molecular weight bioresorbable copolyester called poly (DL-lactide- $\varepsilon$-caprolactone) (B1 and B2). B2 had a rough surface on one side to promote ingrowth in the abdominal wall and prevent migration. The experimental barriers were supplied by the manufacturer (Polyganics, Groningen, The Netherlands) in the appropriate size, $5 \times 5 \mathrm{~cm}$, except for B1 which was supplied in a size of $7 \times 5 \mathrm{~cm}$ and cut to size under sterile conditions. HA-CMC (Seprafilm) was purchased and used according to the manufacturer's instructions. It was cut to a size of $5 \times 5 \mathrm{~cm}$ under sterile conditions.

\section{Animals}

Seventy seven adult, male Wistar rats with a body weight between 200 and $250 \mathrm{~g}$ were obtained from a registered breeding company (Envigo, Horst, The Netherlands) and housed at the central animal facilities of Maastricht University. The animals had free access to food and water, were socially housed with a 12-h dark-light cycle, and cared for according to local protocol. 


\section{Operative procedure}

Preoperatively, all animals received buprenorphine $0.05 \mathrm{mg} / \mathrm{kg}$ and carprofen $4 \mathrm{mg} / \mathrm{kg}$ via subcutaneous injections. Anesthesia was induced with 3\%-4\% isoflurane using an induction chamber. Anesthesia was maintained with $2 \%$ isoflurane. The abdomen was shaved and disinfected with a chlorhexidine solution. The abdomen was opened through a midline incision of approximately $6 \mathrm{~cm}$. On each side of the midline incision, four ischemic buttons were created, $1 \mathrm{~cm}$ lateral of the incision and $1 \mathrm{~cm}$ apart. ${ }^{11-14}$

After creating the buttons, a previously designated experimental barrier, HA-CMC barrier or no barrier was placed according to the randomization. Two barriers (A3 and B2) had one roughened side that was placed facing the abdominal wall. All barriers were fixed to the abdominal wall with two lateral 4-0 polypropylene sutures (Prolene, Ethicon, Johnson \& Johnson, Somerville, NJ) placed in the middle of the barrier on both sides. In case of no barrier placement or an impossibility to fixate by suture, like with HA-CMC barrier which is self-adhesive, two 4-0 polypropylene sutures (Prolene, Ethicon, Johnson \& Johnson, Somerville, NJ) were placed in the abdominal wall lateral of the ischemic buttons. This location corresponds with the fixation of the barrier in the intervention groups.

The abdominal wall was closed with a continuous 4-0 polyglactin suture (Vicryl, Ethicon, Johnson \& Johnson, Somerville, NJ), and the skin was closed intracutaneously with a 4-0 absorbable suture (Monocryl, Ethicon, Johnson \& Johnson, Somerville, NJ).

At the time of sacrifice, the animals were brought under anesthesia according to the previously described protocol, and the abdomen was opened through the scar of the previous midline incision. The adhesions were scored macroscopically, and afterward, the animals were sacrificed by cardiac puncture.

\section{Adhesion scoring}

The adhesions were scored macroscopically using the Nair scoring system for adhesion scoring. ${ }^{15}$ Furthermore, tenacity (Zühlke score) and vascularization of the adhesions were scored (see Table 6.1). ${ }^{16}$ The number of ischemic buttons involved in adhesions and the organ involvement were recorded separately. 
Table 6.1 Adhesion scoring systems.

\begin{tabular}{|c|c|c|}
\hline \multirow[t]{6}{*}{ Nair score } & Grade 0 & No adhesions/ insignificant adhesions \\
\hline & Grade 1 & $\begin{array}{l}\text { Only one adhesions band between the organs or between one organ and } \\
\text { abdominal wall }\end{array}$ \\
\hline & Grade 2 & Two adhesions bands between organs or between one organ and abdominal band \\
\hline & Grade 3 & $\begin{array}{l}\text { More than two adhesion bands between the organs or between one organ and } \\
\text { abdominal wall }\end{array}$ \\
\hline & & or adhesions of intestinal loops without any adhesion to the abdominal wall \\
\hline & Grade 4 & Adhesions of all viscera to the abdominal wall \\
\hline \multirow{5}{*}{$\begin{array}{l}\text { Tenacity score } \\
\text { (Zühlke score) }\end{array}$} & Grade 0 & No adhesions \\
\hline & & \\
\hline & Grade 1 & Filmy adhesions, blunt dissection \\
\hline & Grade 2 & Strong adhesions, sharp dissection \\
\hline & Grade 3 & Very strong vascularized adhesions, sharp dissection, damage hardly preventable \\
\hline Vascularisation & Yes/no & \\
\hline
\end{tabular}

\section{Statistical analysis}

A power calculation was performed, based on the Nair score, estimating an effect size of $20 \%$ with a variance of $16 \%$. To achieve a power of 0.80 with an alfa of 0.05 (twosided test), groups of 11 animals are needed. No drop-out was expected. Nonparametric testing was performed, using the Kruskal-Wallis test with a post hoc Mann-Whitney $U$ test for continuous variables. For nominal variables, a Fisher's exact test was used. Bonferroni correction method was applied to correct for multiple testing; the corrected significance limit was a $P^{*}<0.007(0.05 / 7)$.

\section{Results}

Two rats deceased before the end of the study, one during anesthesia and one during follow-up. The mean preoperative weight was 237.5 gram (SD, 11.5 gram) and did not significantly differ between groups $(P=0.711)$.

\section{Operative procedure}

One of the two deceased animals was allocated to the A1 group and died during anesthesia, by cardiac failure, based on autopsy. In the remaining 76 animals, operative procedures were carried out as planned.

The ease of placement of the different antiadhesive barriers depended on the different materials. B1 appeared to stick to oneself causing difficulty handling the barrier, which complicated intra-abdominal placement. Using the A1 barrier, similar problems were 
encountered, with curling of the barrier. B2, A3, and A2 were considered easy to handle, although the suturing of A2 to the abdominal wall caused tearing of the barrier in two cases.

\section{Follow-up}

The second deceased animal was randomized in the A2 group and was found dead in its cage on the second day post-operatively. No clear cause of death was found, but there were no signs of ileus, perforation, hemorrhage, or intra-abdominal infection at autopsy. In the remaining animals, no complications occurred during follow-up and all completed the 14-day period without reaching humane endpoints.

\section{Macroscopic evaluation}

At sacrifice, the intra-abdominal cavity was inspected, findings were recorded, and remnants of the antiadhesive barrier were examined. A high occurrence of folding of the barrier was observed in the groups receiving the B1, B2, and A1 barriers (see Table 6.2). In 8 of 11 cases, the A2 barrier was torn or fragmented at sacrifice, which was also encountered in one animal after placement of B2.

Table 6.2 Results of macroscopic evaluation presented as median with IQR or percentage of occurrence.

\begin{tabular}{|c|c|c|c|c|c|c|c|c|}
\hline Scoring systems & A1 & $\mathrm{A} 2$ & A3 & B1 & B2 & $\begin{array}{c}\text { HA-CMC } \\
\text { barrier }\end{array}$ & Control & $P$ \\
\hline $\begin{array}{l}\text { Nair score median } \\
\text { (IQR) }\end{array}$ & $3.0(0.0)$ & $3.0(1.0)$ & $3.0(0.5)$ & $3.0(0.0)$ & $3.0(0.0)$ & $3.0(1.0)$ & $3.0(0))$. & 0.274 \\
\hline $\begin{array}{l}\text { Zühlke score Median } \\
\qquad(\mathrm{IQR})\end{array}$ & $2.0(1.0)$ & $1.0(1.25)$ & $2.5(2.0)$ & $3.0(2.0)$ & $1.0(1.0)$ & $2.0(1.0)$ & $2.0(1.0)$ & 0.330 \\
\hline Vascularization & $4 / 9$ & $2 / 10$ & $6 / 11$ & $8 / 11$ & $2 / 11$ & $3 / 11$ & $6 / 11$ & 0.086 \\
\hline Yes/total $(\%)$ & $(44.4 \%)$ & $(20.0 \%)$ & $(54.5 \%)$ & $(72.7 \%)$ & $(18.2 \%)$ & $(27.3 \%)$ & $(54.5 \%)$ & \\
\hline Number of buttons & $5.0(4.5)$ & $3.5(2.25)$ & $4.0(2.25)$ & $6.0(2.0)$ & $5.0(3.0)$ & $6.0(2.0)$ & $8.0(2.0)$ & 0.004 \\
\hline \multicolumn{9}{|l|}{ Median (IQR) } \\
\hline $\begin{array}{l}\text { Number of organs } \\
\text { involved in adhesions } \\
\text { Median (IQR) }\end{array}$ & $2.0(1.0)$ & $1.5(2.0)$ & $2.0(1.5)$ & $2.0(0.0)$ & $2.0(1.0)$ & $3.0(2.0)$ & $3.0(1.0)$ & 0.204 \\
\hline
\end{tabular}

$*=$ not observed; $\mathrm{P}<0.004$ is considered significant.

An extensive adverse reaction was found in one animal in the A2 group. The barrier was encapsulated in a pocket in the abdominal wall extending along the midline incision. This pocket was filled with serous fluid and fibrotic tissue encapsulating the complete but torn A2 barrier (see Figure 6.1). The A3 barrier caused an adverse reaction in three animals. One animal showed an abnormal amount of serous intraabdominal fluid, and one animal presented with thickening and hardening of the abdominal wall caused by fibrotic tissue. In the third animal, a barrier encapsulated by 
fibrotic tissue, serous intra-abdominal fluid and completely adhesive intestines were encountered. Two rats in the B2 group developed a mild reaction with a single cyst on the barrier.
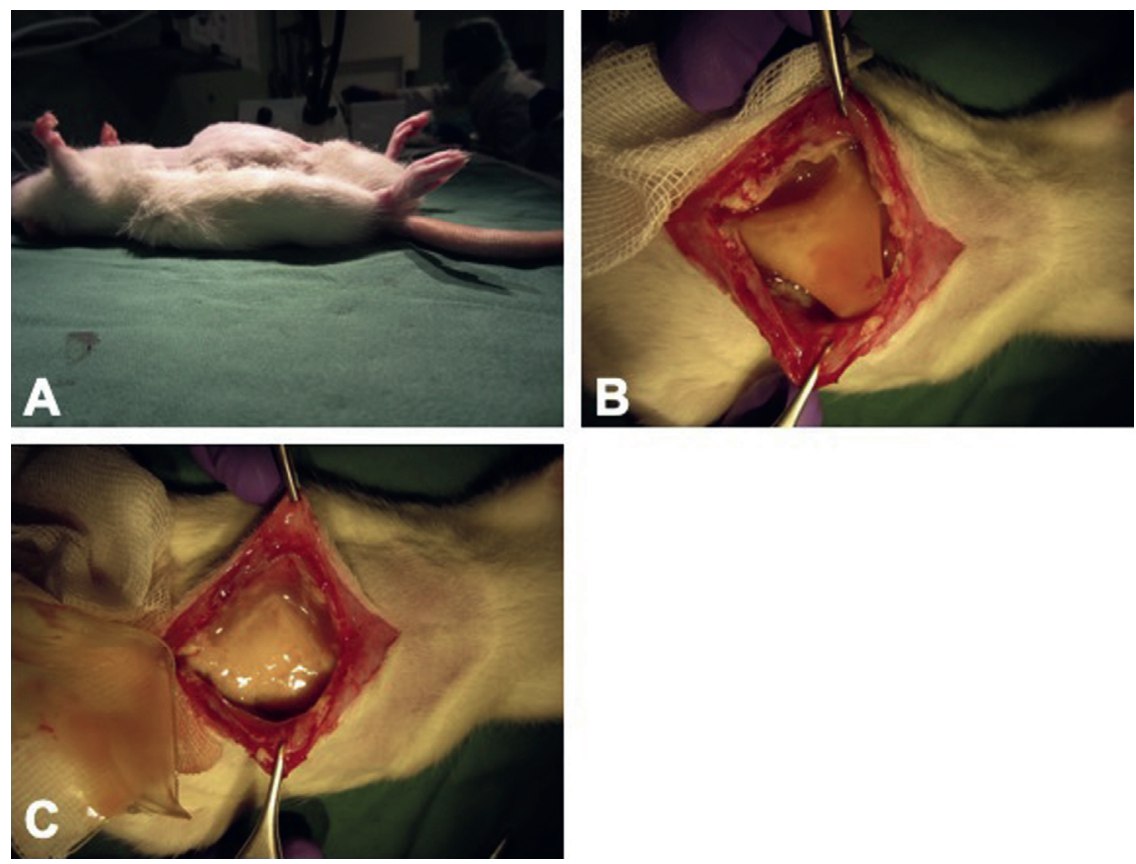

Figure 6.1 One case of a foreign body response in a rat, $14 \mathrm{~d}$ after implantation of the A2 barrier. (A) Macroscopic evaluation $14 \mathrm{~d}$ after surgery, (B) encapsulated barrier encountered after midline incision, (C) cavity filled with fibrotic tissue and serous fluid after removal of the $A 2$ barrier.

In the HA-CMC barrier group, two cases showed an unexpected adverse reaction, one animal showed an inflammatory response in the abdominal wall at sacrifice. In the other animal, a cavity made up of fibrotic tissue of $2 \mathrm{~cm}$ in diameter was encountered distal in the midline incision, which could be explained by a foreign body response although the HA-CMC barrier completely dissolved.

\section{Adhesion assessment}

Adhesions were scored according to the protocol; results are described in Table 6.2. 


\section{Quantity of adhesions}

The median Nair score was 3.0 in all groups, with only differences in the interquartile ranges (IQRs) $(P=0.274)$. The number of ischemic buttons involved in adhesions was the highest in the control group without an antiadhesive barrier with a median of 8.0 (IQR, 2.0), followed by HA-CMC barrier and B1 with both a median of 6.0 ischemic buttons involved (IQR, 2.0). In B2, A1, and A3, 5.0 (IQR, 3.0), 5.0 (IQR, 4.5), and 4.0 (IQR, 2.25) buttons were involved, respectively. The number of buttons involved in adhesions was significantly lower in the A2 group (median, 3.5; IQR, 2.25) than the control group $(P=0.001)$ and $\mathrm{B} 1$ group $(P=0.003)$ but showed no significant difference compared with the HA-CMC barrier group $(P=0.009)$. All other comparisons between groups did not reach statistical significance (see Figure 6.2).

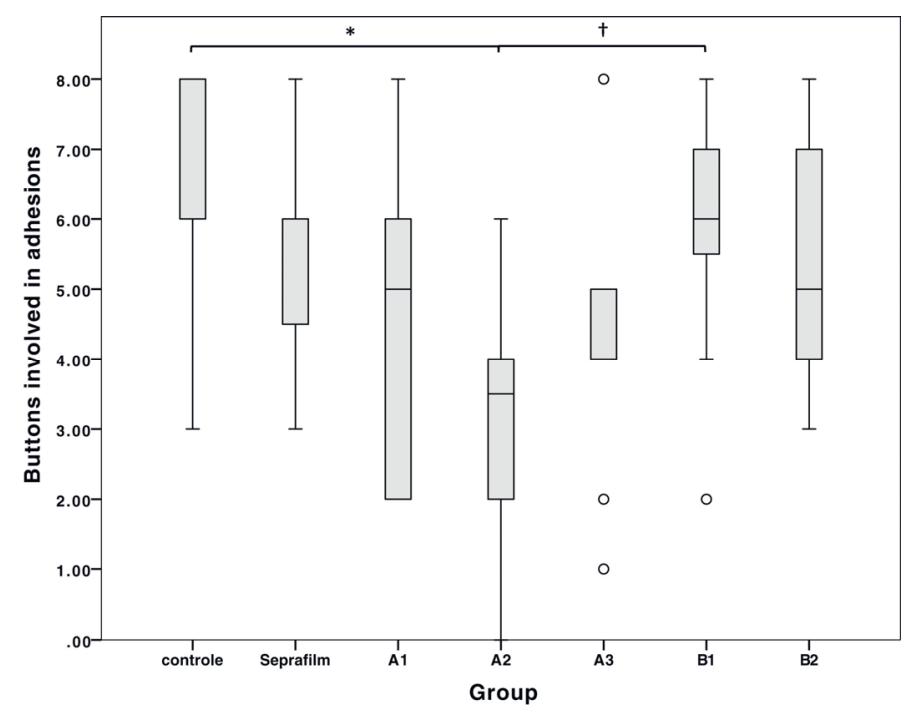

Figure 6.2 Tukey's box plot of number of ischemic buttons involved in adhesion and median with interquartile ranges. Statistics were performed using the Kruskal-Wallis test with a post hoc Mann-Whitney $U$-test. $P^{*}<0.007$ is considered significant. ${ }^{*} P=0.001 ;+P=0.003$.

The number of organs involved in adhesions was recorded as a measure of the extent of adhesion formation, but no significant difference between groups was encountered $(P=0.204)$. One animal in the A2 group showed an adverse reaction to the antiadhesive barrier and subsequently received the maximum Nair score, but all ischemic buttons were incorporated in the pocket encapsulating the antiadhesive barrier. Two of three animals in the A3 group that showed an adverse reaction were scored the maximum Nair score, and eight of eight buttons were involved in adhesions. 


\section{Quality of adhesions}

The median Zühlke score of the adhesions was 3.0 (IQR 2.0) in the B1 group, 2.5 (IQR 2.0) in the A3 group, and 2.0 (IQR 1.0) in both the control and the A1 group. A2, B2, and HA-CMC barriers all scored a median of 1.0 with an IQR of 1.25, 1.0, and 1.0, respectively. No significant differences were found between the groups regarding the Zühlke score $(P=0.330)$, as well as the vascularization of the adhesions $(P=0.086)$. One animal in the A2 group and two animals in the A3 group that were associated with an adverse reaction all scored the maximum Zühlke score.

\section{Discussion}

The burden of adhesions continues to trouble patients after abdominal surgery, not only by complications occurring long after the first operation but also by increasing the risk of complications during reoperation..$^{1-4}$ Unfortunately, the laparoscopic approach does not provide a satisfactory reduction in adhesion formation, ${ }^{2}$ and available antiadhesive barriers fail to produce effective adhesion prevention. ${ }^{8,9}$ Therefore, the search for efficient prevention of adhesion formation is still of great importance.

The adhesions in this animal experiment were scored on two main characteristics; the quantity and quality of the adhesions. The Nair score was used for the evaluation of the quantity of adhesions but failed to distinct between groups. This might be caused by the ischemic button model itself, which induces extensive adhesion formation, leading in almost all cases to a Nair score of three (more than two adhesion bands present). The same has been described in a previous report. ${ }^{14}$ The Nair score lacks finesse in this model in contrast to for instance the cecal abrasion model, where a more confined area is scored for adhesions. Scoring the four quadrants of the abdomen separately would increase the precision but would also allow room for error because of the small size of the abdomen of the rat.

The number of buttons involved in adhesions appears to be a more suitable outcome for adhesion evaluation in this model. The fact that the median number of buttons involved in adhesions was eight of eight buttons in the control group proves that the ischemic button model is sufficiently adequate in adhesion induction. The A2 barrier showed a significantly lower number of ischemic buttons involved in adhesions than no antiadhesive barrier and the B1 barrier. Regarding the quality of the adhesion, the Zühlke score, and vascularization of the adhesions, we could not detect any significant differences between groups. 
Based on the lower number of buttons involved in adhesions in the A2 group, a better performance regarding adhesion reduction can be concluded. In three antiadhesive barriers, A2, A3, and B2, an adverse reaction was encountered at sacrifice. In the B2 and in two cases of the A3 group, the adverse reaction could be classified as mild. But in one case in the A3 and the A2 group, an extensive adverse reaction occurred with encapsulation of the barrier. In addition, one of the animals in the A2 group was found dead during follow-up and no cause of death could be established. This could be an incidental finding, but a relation to the implantation of the A2 barrier cannot be ruled out. In case of the latter, two adverse reactions related to A2 implantation were recorded. B2 had a sanded surface on one side to promote attachment to the abdominal wall, and A3 was enriched with bioactive glass to enhance antibacterial qualities. Interestingly, these two barriers both had a rough surface facing the abdominal wall, which might be involved in the development of an adverse reaction.

There are limitations to this study worth mentioning. Despite all measures to ensure random allocation, blinding of allocation, and blind outcome assessment, the nature of the different sheets and control groups rendered absolute blinding impossible, which could be a source for bias. All antiadhesive barriers investigated were not self-adherent and in need of additional fixation. The barrier was fixated with two nonabsorbable sutures positioned midlateral at the barrier. Sutures as a fixation method are known to induce adhesions in the presence of an already damaged peritoneum. ${ }^{17}$ Nonabsorbable sutures are equally associated with intra-abdominal adhesions in terms of incidence and severity compared with absorbable sutures when used for mesh fixation after 1 week follow-up. ${ }^{18}$ In this experiment, two nonabsorbable sutures were placed in all intervention groups, including in the self-adherent HA-CMC barrier group, equalizing the adhesion-inducing effect of sutures. The lack of self-adhering qualities of the investigated barriers allows for space between the barrier and the abdominal wall, which can be occupied by mobile organs such as the omentum or scrotal fat. The antiadhesive barrier function is bypassed, and adhesions are allowed to form.

The positioning of the sutures midlaterally in the barrier is also a point of discussion. The use of more sutures, such as one in every corner, would induce more adhesions interfering with the model, but the two-suture fixation method allowed for folding of the barrier. This kind of migration of the barrier might disadvantage the coverage of the ischemic buttons and reduce the antiadhesive barrier function.

The HA-CMC barrier performed equally and, in most cases, worse than the intervention barriers, which was unexpected. Especially, regarding the number of ischemic buttons and organs involved in adhesions, the HA-CMC barrier did not achieve the anticipated results. The ischemic button might be a very fiercely adhesioninducing model overstraining the antiadhesive capacity of the HA-CMC barrier. In a 
previous report, however, significant adhesion reduction by a $5 \times 7 \mathrm{~cm}$ HA-CMC barrier in an ischemic button model in a comparable setting was accomplished. Despite the difference in size, adequate covering of the ischemic buttons was achieved in this experiment. Placing eight buttons instead of six appears to be of high impact on the adhesion formation. ${ }^{19}$

\section{Conclusion}

The A2 barrier showed a significant reduction in adhesion formation in contrast to the remaining four polymer antiadhesive barriers tested in this ischemic button model. The encountered extensive adverse events in the A2 group raise serious doubt on its applicability. The Nair score lacks sensitivity and appears inappropriate for adhesion assessment in the ischemic button model. Given the adhesion-inducing capacities of suture material and chance of migration, future studies might focus on the self-adhering properties of barriers. 


\section{References}

1. Menzies $\mathrm{D}$, Ellis $\mathrm{H}$. Intestinal obstruction from adhesions--how big is the problem? Ann R Coll Surg Engl. 1990;72(1):60-3.

2. Stommel MWJ, Ten Broek RPG, Strik C, Slooter GD, Verhoef C, Grunhagen DJ, et al. Multicenter Observational Study of Adhesion Formation After Open-and Laparoscopic Surgery for Colorectal Cancer. Ann Surg. 2018;267(4):743-8.

3. ten Broek RP, Issa Y, van Santbrink EJ, Bouvy ND, Kruitwagen RF, Jeekel J, et al. Burden of adhesions in abdominal and pelvic surgery: systematic review and met-analysis. BMJ. 2013;347:f5588.

4. ten Broek RP, Strik C, Issa Y, Bleichrodt RP, van Goor H. Adhesiolysis-related morbidity in abdominal surgery. Ann Surg. 2013;258(1):98-106.

5. Parker MC, Ellis H, Moran BJ, Thompson JN, Wilson MS, Menzies D, et al. Postoperative adhesions: ten-year follow-up of 12,584 patients undergoing lower abdominal surgery. Dis Colon Rectum. 2001;44(6):822-29; discussion 9-30.

6. Parker MC, Wilson MS, Menzies D, Sunderland G, Clark DN, Knight AD, et al. The SCAR-3 study: 5-year adhesion-related readmission risk following lower abdominal surgical procedures. Colorectal Dis. 2005;7(6):551-8.

7. Andersen P, Jensen KK, Erichsen R, Froslev T, Krarup PM, Madsen MR, et al. Nationwide populationbased cohort study to assess risk of surgery for adhesive small bowel obstruction following open or laparoscopic rectal cancer resection. BJS Open. 2017;1(2):30-8.

8. Ten Broek RPG, Stommel MWJ, Strik C, van Laarhoven C, Keus F, van Goor H. Benefits and harms of adhesion barriers for abdominal surgery: a systematic review and meta-analysis. Lancet. 2014;383(9911):48-59.

9. Kumar S, Wong PF, Leaper DJ. Intra-peritoneal prophylactic agents for preventing adhesions and adhesive intestinal obstruction after non-gynaecological abdominal surgery. Cochrane Database Syst Rev. 2009(1):CD005080.

10. van Steensel S, van den Hil LCL, Schreinemacher MHF, Ten Broek RPG, van Goor H, Bouvy ND. Adhesion awareness in 2016: An update of the national survey of surgeons. PLoS One. 2018;13(8):e0202418.

11. Kraemer B, Wallwiener C, Rajab TK, Brochhausen C, Wallwiener M, Rothmund R. Standardised models for inducing experimental peritoneal adhesions in female rats. Biomed Res Int. 2014;2014:435056.

12. Rajab TK, Wauschkuhn CA, Smaxwil L, Kraemer B, Wallwiener M, Wallwiener CW. An improved model for the induction of experimental adhesions. J Invest Surg. 2010;23(1):35-9.

13. Whang SH, Astudillo JA, Sporn E, Bachman SL, Miedema BW, Davis W, et al. In search of the best peritoneal adhesion model: comparison of different techniques in a rat model. J Surg Res. 2011;167(2):245-50.

14. Mommers EH, Hong L, Jongen A, Bouvy ND. Baseline performance of the ischaemic button model for induction of adhesions in laboratory rats. Lab Anim. 2019;53(1):63-71.

15. Nair SK, Bhat IK, Aurora AL. Role of proteolytic enzyme in the prevention of postoperative intraperitoneal adhesions. Arch Surg. 1974;108(6):849-53.

16. Zuhlke HV, Lorenz EM, Straub EM, Savvas V. [Pathophysiology and classification of adhesions]. Langenbecks Arch Chir Suppl II Verh Dtsch Ges Chir. 1990:1009-16.

17. Wallwiener CW, Kraemer B, Wallwiener M, Brochhausen C, Isaacson KB, Rajab TK. The extent of adhesion induction through electrocoagulation and suturing in an experimental rat study. Fertil Steril. 2010;93(4):1040-4.

18. Schreinemacher MH, van Barneveld KW, Peeters E, Miserez M, Gijbels MJ, Greve JW, et al. Adhesions to sutures, tackers, and glue for intraperitoneal mesh fixation: an experimental study. Hernia. 2014;18(6):865-72.

19. Vogels RR, Bosmans JW, van Barneveld KW, Verdoold V, van Rijn S, Gijbels MJ, et al. A new poly(1,3trimethylene carbonate) film provides effective adhesion reduction after major abdominal surgery in a rat model. Surgery. 2015;157(6):1113-20. 
Chapter 6 


\title{
Chapter 7
}

\author{
Prevention of intra-abdominal adhesions by a \\ hyaluronic acid gel; an experimental study in rats
}

Sebastiaan van Steensel, Hong Liu, Timon F.G. Vercoulen, M'hamed Hadfoune, Stephanie O. Breukink, Laurents P.S. Stassen, Kaatje Lenaerts, Nicole D. Bouvy 


\section{Abstract}

\section{Background}

In $80 \%$ to $90 \%$ of the patients intra-abdominal adhesions occur after abdominal surgery, which can cause small-bowel obstruction, chronic abdominal pain, female infertility and difficulty during reoperation. A novel crosslinked hyaluronic acid gel is evaluated regarding its antiadhesive capacities in an ischemic button model in rats.

\section{Methods}

51 adult, male Wistar rats from a registered breeder, received eight ischemic buttons each and were treated with hyaluronic acid gel (HA, HyaRegen ${ }^{\odot}$ ), hyaluronic acid carboxymethylcellulose (HA-CMC, Seprafilm ${ }^{\circ}$ ) or no antiadhesive barrier. After 14 days, the animals were sacrificed and adhesions were scored macroscopically. The number of buttons and organs involved in adhesions were recorded. Per animal, one button with adhesions and one without adhesions was explanted for qPCR analysis. Mann-Whitney U, Fisher's exact and Wilcoxon signed rank test were used for data analysis. A $P$-value of 0.05 was considered significant.

\section{Results}

Macroscopic evaluation of adhesion formation did not differ between the groups. The number of organs involved in adhesions in the HA gel group was significantly lower compared to HA-CMC $(P=0.041)$ and the control group $(P=0.012)$. A significantly, 1.36-fold higher clec10a $(P=0.25), 1.80$-fold higher $\operatorname{cd} 163(P=0.003)$ and 5.14-fold higher mmpl expression $(P=0.028)$ was found in ischemic buttons with adhesions compared to buttons without adhesions.

\section{Conclusion}

HA gel application reduces the number of organs involved in adhesions in an ischemic button model, but no overall reduction in adhesion formation was encountered. Macrophage subtype 2 polarization and high $\mathrm{mmpl}$ expression are associated with adhesion formation. Further investigation is needed in the exact pathophysiologic process of adhesion formation and the role of macrophage polarization. 


\section{Introduction}

Intra-abdominal adhesion formation is the most frequent occurring complication following surgery of the abdomen, with an incidence reported of $80 \%$ to $90 \%$. ${ }^{1,2}$ The risk of morbidity, which is directly related to adhesions, is $3.8 \%$ after abdominal surgery accompanied by an average rate of readmission of $2.1 .^{3,4}$ Of small-bowel obstructions, $56 \%$ is caused by adhesions. In $57 \%$ of patients with chronic abdominal pain, adhesions are considered responsible, and $23 \%$ of female patients seek fertility treatment after abdominal surgery. ${ }^{5}$ Apart from these complications, future surgery is hampered by adhesions and adhesiolysis is needed with an increased risk of iatrogenic bowel injury. ${ }^{5,6}$ Consequently, adhesiolysis leads to an increased incidence of sepsis, intra-abdominal complications and wound infections resulting in longer hospital stay and higher hospital costs. ${ }^{6}$

Laparoscopic surgery is associated with less intra-abdominal adhesions than conventional surgery, although $37.7 \%$ of the laparoscopic operated patients still develop postoperative adhesions. A surgical intervention to treat adhesions is therefore not preferable and prevention of adhesions using antiadhesive barriers might offer a better strategy. Two such barriers, oxidised regenerated cellulose (Interceed ${ }^{\odot}$, Ethicon, US, NJ) and hyaluronate carboxymethylcellulose (HA-CMC, Seprafilm ${ }^{\odot}$, Sanofi, US, $\mathrm{NJ}$ ) are suggested to be effective in reducing adhesion formation. Also, in case of HA$\mathrm{CMC}$, a reduction of reoperations for small bowel obstruction is reported. ${ }^{7,8}$ The only available liquid antiadhesive barrier, icodextrin 4\% (Adept, Baxter Healthcare, Deerfield, IL) is afflicted by limited research and does not show a beneficial effect on the reoperation rate for small bowel obstruction. ${ }^{8}$

HA-CMC has a beneficial effect on the severity of the adhesions, but the extent of adhesion formation is merely reduced by $25.9 \%$. This translates in a reduction of the reoperation rate, although the incidence of small-bowel obstruction is not altered after HA-CMC application. ${ }^{7,8}$ These results are reflected by the opinion of Dutch surgeons in a National survey, where responders were reluctant in using antiadhesive barriers. ${ }^{9}$ Furthermore, is Seprafilm, when wrapped around an anastomosis of the bowel, associated with a higher occurrence of anastomotic leakage and its consequences, such as fistula, peritonitis, abscess and sepsis. ${ }^{10}$

The pathophysiological process of the formation of adhesions is characterized by a complex interaction between different factors, such as fibrin deposition and failure of the fibrinolytic system, macrophages and fibroblasts. ${ }^{11-15}$ Isolated pathways are described, but the interaction between these components is poorly understood. A continuum between two polarized states of macrophage activation is described in the macrophage M1/M2 paradigm. Macrophage subtype 1 (M1) is recognized as a pro- 
inflammatory macrophage or classical macrophage and is associated with tissue injury. Macrophage subtype 2 (M2) defines the other side of the spectrum and is characterized by more anti-inflammatory properties including extracellular matrix remodeling and fibroblast regulation. ${ }^{16-18}$ In this animal experiment the secondary aim is to investigate the pathophysiological process of adhesion formation. Genes of interest in regard to adhesion formation consist of genes associated with macrophage subtype 1 and 2, fibroblast activation and adhesion specific genes.

The quest for an optimal antiadhesive barrier continues. The primary aim of this study is to evaluate the effect of a hyaluronic acid-based gel on the intra-abdominal adhesion formation in the ischemic button model in rats and to compare its effect to an already commercially available antiadhesive barrier (HA-CMC). To be of added value in clinical practise, the novel antiadhesive barrier should be superior to what is currently available for clinicians in the prevention of adhesions.

\section{Materials and methods}

The ethical committee of animal experiments approved the study protocol (AVD107002016720), which complied to the Dutch Animal Experimental Act and the European Directive 2010/63/EU.

\section{Materials}

Crosslinked hyaluronic acid gel, HyaRegen ${ }^{\odot}$ (HA) is produced by BioRegen Biomedical Co., Ltd. (Changzhou, China) and showed promising results regarding adhesion prevention in the gynaecological patient. ${ }^{19}$ The product was delivered in $20 \mathrm{cc}$ sterile syringes and a $3 \mathrm{cc}$ volume was applied intra-abdominally in the rat. Hyaluronate carboxymethylcellulose, Seprafilm ${ }^{\odot}$ (HA-CMC) was acquired commercially and under sterile conditions, cut to a size of $5 \times 5 \mathrm{~cm}$ after which it was placed on top of the intestines.

\section{Animals}

51 adult, male Wistar rats with a body weight between 200-250 g were obtained from a registered breeding company (Envigo, Horst, the Netherlands). All animals were accommodated at the central animal facilities of Maastricht University and cared for according to local protocol, with free access to food and water, a 12-hour dark/light cycle and social housing. 


\section{Study design}

Due to the obvious difference between HA-CMC and HA gel, blinding of the surgeon was not possible at the time of surgery. The animals were randomly placed in one of three groups ( $n=17$ per group); HA gel, HA-CMC or control group. The animals in the control group did receive 8 ischemic buttons but did not receive any antiadhesive barrier. It was made sure that in each cage every group was equally represented, with three animals per cage. After a 14-day observation period the animals were sacrificed.

\section{Surgical procedure}

Pre-operative pain medication was administered via subcutaneous injection of buprenorphine $0.05 \mathrm{mg} / \mathrm{kg}$ and carprofen $4 \mathrm{mg} / \mathrm{kg}$. The animal was placed in an induction chamber to induce anaesthesia via inhalation with $3-4 \%$ isoflurane. Anaesthesia was maintained with $2 \%$ isoflurane. Before surgery, the abdomen was shaved and disinfected with chlorhexidine. Access to the abdomen was gained through a $6 \mathrm{~cm}$ midline incision and on either side of the midline incision four ischemic buttons were created. ${ }^{20-22}$ In short, $5 \mathrm{~mm}$ of parietal peritoneum was lifted and ligated with a non-absorbable suture (Prolene, Ethicon, Johnson \& Johnson, Somerville, NJ) to create the ischemic button. All animals, regardless of group allocation, received eight ischemic buttons. The button was placed $1 \mathrm{~cm}$ lateral of the incision and at least $1 \mathrm{~cm}$ apart from one another. Thereafter, HA-CMC or $3 \mathrm{ml}$ of HA gel, was left behind according to group allocation and in case of the control group, no product was used. No suturing nor fixation took place, because HA-CMC is self-adherent. A continuous 4-0 polyglactin suture (Vicryl, Ethicon, Johnson \& Johnson, Somerville, NJ) was used for closure of the abdominal wall after which the skin was intracutaneously closed using an absorbable Monocryl 4-0 suture (Ethicon, Johnson \& Johnson, Somerville, NJ). The animals received fluid resuscitation postoperatively and were allowed to recover under a heat lamp. Careful welfare assessment was conducted using welfare score sheets during follow-up.

After 14 days of follow-up a second operation took place for the evaluation of intraabdominal adhesion formation. The previously described protocol was followed regarding preoperative pain management and the anaesthesia. The abdomen was accessed through the midline scar and any complications or unexpected observations were recorded. Next, the intra-abdominal adhesions were scored macroscopically. If present, one ischemic button with attached adhesions and one ischemic button which failed to induce adhesions was collected per animal and snap frozen for qPCR analysis. The animals were sacrificed by cardiac puncture while still sedated after completion of the procedure. 


\section{Adhesion scoring}

Adhesions were scored macroscopically regarding the quantity and quality of the adhesions. The quantity of adhesions was assessed using the Nair score, ${ }^{23}$ both the number of ischemic buttons involved in adhesions and the number of intra-abdominal organs involved in adhesions were scored. The tenacity (Zühlke score ${ }^{24}$ ) and vascularisation of the present adhesions were a measure for the quality of adhesions. See Table 7.1 for the scoring system.

Table 7.1 Adhesion scoring systems.

\begin{tabular}{|c|c|c|}
\hline Nair score & Grade 0 & No adhesions/ insignificant adhesions \\
\hline & Grade 1 & $\begin{array}{l}\text { Only one adhesions band between the organs or between one organ and } \\
\text { abdominal wall }\end{array}$ \\
\hline & Grade 2 & $\begin{array}{l}\text { Two adhesions bands between organs or between one organ and abdominal } \\
\text { band }\end{array}$ \\
\hline & Grade 3 & $\begin{array}{l}\text { More than two adhesion bands between the organs or between one organ and } \\
\text { abdominal wall } \\
\text { or adhesions of intestinal loops without any adhesion to the abdominal wall }\end{array}$ \\
\hline & Grade 4 & Adhesions of all viscera to the abdominal wall \\
\hline \multirow{4}{*}{$\begin{array}{l}\text { Tenacity score } \\
\text { (Zühlke score) }\end{array}$} & Grade 0 & No adhesions \\
\hline & Grade 1 & Filmy adhesions, blunt dissection \\
\hline & Grade 2 & Strong adhesions, sharp dissection \\
\hline & Grade 3 & $\begin{array}{l}\text { Very strong vascularized adhesions, sharp dissection, damage hardly } \\
\text { preventable }\end{array}$ \\
\hline
\end{tabular}

\section{Evaluation by expert panel}

In order to directly link this animal experiment to clinical application, three clinical experts were asked to evaluate adhesion formation in the animals. During the macroscopic scoring digital photographs were taken from either side of the midline. Three abdominal surgeons (SB, LS, NB) were blinded to the allocation of the animals and asked to score both the quantity and the quality of adhesions (see Table 7.2). Both sides of the midline incision were scored separately, generating two scores regarding quantity and quality of adhesions per animal. Per expert, the scores were added and the total scores from the three experts were averaged and reported. The used scoring systems are regularly used in other animal adhesion models. ${ }^{22,25-28}$ 
Table 7.2 Scoring of adhesions by experts.

\begin{tabular}{ll}
\hline Quantity score: & What percentage of the surface of the abdominal wall is involved in adhesions? \\
\hline 0 & $0 \%$ \\
1 & $<25 \%$ \\
2 & $25-49 \%$ \\
3 & $50-74 \%$ \\
4 & $75-100 \%$ \\
Quality score: & How would you describe the \\
0 & severity of the adhesions? \\
1 & No adhesions visible \\
2 & Filmy, avascular adhesions \\
3 & Moderate thickness, limited vascularity \\
\hline
\end{tabular}

\section{RNA isolation and quantitative real time PCR}

Samples from animals from which a button with adhesions and a button without adhesions was available, were selected for qPCR analysis. Abdominal wall tissue specimen was collected and snap frozen, from which total RNA was isolated using TRI reagent (Sigma, NL). $750 \mathrm{ng}$ DNAse-treated RNA was used to synthesize cDNA (SensiFAST $^{\mathrm{TM}}$, cDNA synthesis kit, Bioline, London, UK). A volume of $10 \mathrm{ml}$ consisting of the cDNA equivalent of $2.5 \mathrm{ng}$ total RNA, 1 Absolute qPCR SYBR Green Fluorescein Mix (SensiFAST ${ }^{\mathrm{TM}} \mathrm{SYBR}^{\circledR} \mathrm{Hi}-\mathrm{ROX}$ Kit, Bioline, London, UK) and $0.15 \mathrm{mM}$ of gene-specific primers (Sigma, NL) was used for qPCR reactions. The LightCycler $^{(B)} 480$ Instrument II (Roche Molecular Systems, Inc) was used to perform the qPCR. Gene expression levels were established with LinRegPCR software. The geometric mean of two internal control genes (Rplp0 and beta-actin (Actb)) was calculated and used as normalization factor. Relevant primers were identified from the literature and build using a primer design tool (Primer-blast), ${ }^{29}$ the sequences are reported in Table 7.3. All primers were tested for transcription of the intended gene.

\section{Statistical analysis}

Sample-size calculation was based on previous research with an ischemic button model within our research group. An effect size of 1.11 with a reduction of $30 \%$ of involved ischemic buttons in adhesions was considered clinically relevant. This resulted in a sample-size of 15 animals per group with a power of 0.8 and an alpha of 0.05 . A $10 \%$ drop-out was taken into account resulting in 17 animals per group.

Values were expressed as median with interquartile ranges or in proportions. Given the small sample size, non-parametric testing was performed using the Mann-Whitney $U$ test for continues and ordinal variables. Nominal variables were analysed with a Fisher's exact test. In case of dependent samples, a Wilcoxon signed rank test was 
performed. A p-value of 0.05 was considered significant. Because only the intervention group was compared to either the control group or HA-CMC group no correction for multiple testing was applied. Data-analysis was performed with SPSS 23.0 for Mac (SPSS Inc, Chicago, IL).

Table 7.3 Primers used for qPCR analysis.

\begin{tabular}{|c|c|c|c|c|}
\hline Gene symbol (name) & $\begin{array}{l}\text { Product } \\
\text { length }\end{array}$ & & $\mathrm{GC} \%$ & sequence \\
\hline \multirow{2}{*}{\multicolumn{2}{|c|}{$\begin{array}{l}\text { rplp0 (ribosomal protein lateral stalk subunit } 190 \\
\text { P0) }\end{array}$}} & $\mathrm{f}$ & 55.00 & CCTCACCGAGATTAGGGACA \\
\hline & & $\mathrm{r}$ & 45.00 & ATCGCTCAGGATTTCAATGG \\
\hline \multirow[t]{2}{*}{ actb (actin, beta) } & 297 & $\mathrm{f}$ & 55.00 & CCGCGAGTACAACCTTCTTG \\
\hline & & $\mathrm{r}$ & 55.00 & CAGTTGGTGACAATGCCGTG \\
\hline \multirow[t]{2}{*}{ i16 (Interleukin 6) } & 246 & $\mathrm{f}$ & 57.14 & CTCTCCGCAAGAGACTTCCAG \\
\hline & & $\mathrm{r}$ & 47.62 & TTCTGACAGTGCATCATCGCT \\
\hline \multirow[t]{2}{*}{$\operatorname{nos} 2$ (iNOS) } & 234 & $\mathrm{f}$ & 52.38 & TAGTCAACTACAAGCCCCACG \\
\hline & & $\mathrm{r}$ & 60 & GTGAGGAACTGGGGGAAACC \\
\hline \multirow[t]{2}{*}{ cd86 (CD86) } & 164 & $\mathrm{f}$ & 45.45 & AGACATGTGTAACCTGCACCAT \\
\hline & & $\mathrm{r}$ & 55 & TACGAGCTCACTCGGGCTTA \\
\hline \multirow[t]{2}{*}{ il10 (Interleukin 10) } & 186 & $\mathrm{f}$ & 52.38 & CGACGCTGTCATCGATTTCTC \\
\hline & & $\mathrm{r}$ & 60.00 & CAGTAGATGCCGGGTGGTTC \\
\hline \multirow[t]{2}{*}{ clec10a (C-type lectin domain containing 10a) } & 164 & $\mathrm{f}$ & 60.00 & GAGGCTTGAGCCAGAAGGTG \\
\hline & & $\mathrm{r}$ & 52.38 & TGCTGAGCCGTTGTTCTTGAG \\
\hline \multirow[t]{2}{*}{ mrc1 (mannose receptor C typ 1 ) } & 212 & $\mathrm{f}$ & 60.00 & CCCGCTCCTCAAGACAATCC \\
\hline & & $\mathrm{r}$ & 55.00 & AAATACGGTGACTGCCCACC \\
\hline \multirow[t]{2}{*}{$\operatorname{cd} 163(\mathrm{CD} 163)$} & 131 & $\mathrm{f}$ & 60 & CTCTGAAGCGACGACAGACC \\
\hline & & $\mathrm{r}$ & 50 & ATGCCAACCCGAGGATTTCA \\
\hline \multirow[t]{2}{*}{ tgfb1 (transforming growth factor-b) } & 115 & $\mathrm{f}$ & 60.00 & GGCTGAACCAAGGAGACGGA \\
\hline & & $\mathrm{r}$ & 55.00 & CCTCGACGTTTGGGACTGAT \\
\hline \multirow[t]{2}{*}{ Infg (Interferon gamma) } & 128 & $\mathrm{f}$ & 50 & CAACCAGGCCATCAGCAACAACAT \\
\hline & & $\mathrm{r}$ & 50 & TCTGTGGGTTGTTCACCTCGAACT \\
\hline \multirow[t]{2}{*}{ Serpine1 (pai-1) } & 123 & $\mathrm{f}$ & 55.00 & CGTCTTCCTCCACAGCCATT \\
\hline & & $\mathrm{r}$ & 55.00 & GCTGGCCCATGAAGAGGATT \\
\hline \multirow[t]{2}{*}{ Serpineb2 (pai-2) } & 223 & $\mathrm{f}$ & 52.38 & AGCCGCTCAGAAGATAACGAG \\
\hline & & $\mathrm{r}$ & 39.13 & CAAAATTCAGCACTTTGGCCATT \\
\hline \multirow[t]{2}{*}{ colla1 (collagen type 1 alpha 1 chain) } & 237 & $\mathrm{f}$ & 60 & CTGACTGGAAGAGCGGAGAG \\
\hline & & $\mathrm{r}$ & 55.00 & CAGGATCGGAACCTTCGCTT \\
\hline \multirow[t]{2}{*}{ mmp1 (matrix metallopeptidase 1) } & 144 & $\mathrm{f}$ & 55.00 & AAGGCCACTGGTGATCTTGC \\
\hline & & $\mathrm{r}$ & 43.48 & GGTATTTCCAGACTGTTTCCACA \\
\hline \multirow[t]{2}{*}{ fn1 (Fibronectin 1) } & 165 & $\mathrm{f}$ & 63.16 & TCCCCTCCCAGAGAAGTGG \\
\hline & & $\mathrm{r}$ & 43.48 & TTGGGGAAGCTCATCTGTCTTTT \\
\hline
\end{tabular}




\section{Results}

Of the 51 animals, one animal in the HA-CMC group died during the operation related to anaesthesia. The remaining 50 animals completed a follow-up of 14 days as planned. The mean weight of the animals prior to surgery was 232.9 gr (SD $12.3 \mathrm{gr}$ ), this was not significantly different between the groups $(P=0.816)$.

\section{Macroscopic evaluation}

Results of macroscopic evaluation are described in Table 7.4. Quantity of adhesions was assessed based on the Nair score, number of buttons and number of organs involved in adhesions. The Nair score was equal between all groups with a median of 3.0 (IQR 1.0). Median number of involved buttons was not different when comparing HA gel (median 7.0, IQR 3.0) to the control group (median 7.0, IQR 1.0, $P=0.067$ ) or to the HA-CMC group (median 7.0, IQR 1.5, $P=0.451$ ). Regarding the number of organs involved in adhesions the median was 2.0 in all groups, but the interquartile range was 0.0 in the HA group compared to 1.0 in the HA-CMC and control group. Number of organs involved in adhesions in the HA group was significantly different compared to the HA-CMC $(P=0.041)$ and the control group $(P=0.012)$. The greater omentum was involved in adhesions in all the animals and scrotal fat in $82.4 \%$ of the animals in the HA gel group, $93.8 \%$ of the animals in the HA-CMC group and $94.1 \%$ of the animals in the control group $(P=0.600)$. Organs other than the greater omentum or scrotal fat were involved in $11.8 \%, 37.5 \%$ and $47.1 \%$ of the animals respectively $(P=0.069)$.

Table 7.4 Results of macroscopic adhesion scoring presented as median with IQR or percentage of occurrence in comparison to HA gel.

\begin{tabular}{|c|c|c|c|c|c|}
\hline & HA gel & HA-CMC & & Control & \\
\hline Nair score Median (IQR) & $3.0(1.0)$ & $3.0(1.0)$ & $P=0.950$ & $3.0(1.0)$ & $P=0.138$ \\
\hline Tenacity (Zühlke score) & $2.0(1.0)$ & $2.0(1.0)$ & $P=0.878$ & $2.0(1.0)$ & $P=0.918$ \\
\hline \multicolumn{6}{|l|}{ Median (IQR) } \\
\hline Vascularisation yes/total (\%) & $0 / 17(0.0 \%)$ & $1 / 16$ & $(6.3 \%)$ & $2 / 17(11.8 \%)$ & \\
\hline $\begin{array}{l}\text { Number of buttons Median } \\
\text { (IQR) }\end{array}$ & $7.0(3.0)$ & $7.0(1.5)$ & $P=0.451$ & $7.0(1.0)$ & $P=0.051$ \\
\hline $\begin{array}{l}\text { Number of organs involved in } \\
\text { adhesions Median (IQR) }\end{array}$ & $2.0(0.0)$ & $2.0(1.0)$ & $P=5.041$ & $2.0(1.0)$ & $P=5.012$ \\
\hline
\end{tabular}

$P$ values are related to the comparison of the HA group to one of the control groups (Mann-Whitney $U$ test).

Tenacity and the vascularisation of the adhesions define the quality of the adhesions. The tenacity was not different between HA gel and the other two groups with a median of 2.0 (IQR 1.0). There was no vascularisation of adhesions observed in the HA gel 
group, in one animal in the HA-CMC group (6.3\%) and in two animals in the control group (11.8\%). The difference was not significant between the groups.

\section{Expert panel}

Three abdominal surgeons, blinded to the allocation of the animals scored the photographs taken after sacrifice, (See Table 7.5). The total adhesion score was highest when HA-CMC was used (median 8.0, IQR 4.8, $P=0.179$ ) followed by the rats in the control group (median 7.0, IQR 2.0, $P=0.734$ ). HA gel group showed the lowest score with a median of 6.3 (IQR 3.7); this did not reach significance compared to the other groups.

Table 7.5 Results of adhesions scoring by three experts, both sides of the midline incision were scored separately leading to a maximum score of 8 regarding quantity, 6 regarding quality and a maximum total score of 14 per animal. Median with IQR are presented.

\begin{tabular}{|c|c|c|c|c|c|}
\hline & HA gel & HA-CMC & & Control & \\
\hline Quantity of adhesions & $3.0(2.7)$ & $4.0(2.8)$ & $P=0.146$ & $3.33(1.5)$ & $P=0.708$ \\
\hline Median (IQR) & & & & & \\
\hline $\begin{array}{l}\text { Quality of adhesions Median } \\
\text { (IQR) }\end{array}$ & $3.3(1.2)$ & $4.0(2.2)$ & $P=0.276$ & $3.7(0.7)$ & $P=0.946$ \\
\hline Total score Median (IQR) & $6.3(3.7)$ & $8.0(4.8)$ & $P=0.179$ & $7.0(2.0)$ & $P=0.734$ \\
\hline
\end{tabular}

Comparison of HA gel to HA-CMC and to the control group, $\mathrm{p}$-values are expressed in relation to the HA group.

Quantity of adhesions was scored lowest in the HA animals with a median of 3.0 (IQR 2.7). This was not significantly lower compared to HA-CMC (median 4.0, IQR 2.8, $P=0.146$ ) and the control group (median 3.33, IQR 1.5, $P=0.708$ ). Quality of adhesions was also scored lowest after the use of HA gel (median 3.3, IQR 1.2) compared to HACMC (median 4.0, IQR 2.2, $\mathrm{P}=0.276$ ) and the control group (median 3.7, IQR 0.7, $P=0.946)$.

\section{qPCR}

The qPCR was used to determine the role of macrophage polarisation in the formation of adhesions. Gene expression characteristic for subtype one (M1: il6, nos2, cd86, and infg) and subtype two (M2: il10, clec10a, mrc1, cd163, and tgfb1) were both investigated to provide information regarding dominant subtype presence in the tissue. M1 is associated with pro-inflammatory properties in contrast to M2, which plays a role in extra-cellular matrix remodeling and regulation of fibroblast. ${ }^{16-18}$ In this experiment, it is hypothesised that a dominant presence of M2 is involved in the formation of adhesions. In addition, the expression of genes typically active in 
fibroblasts (collal, mmpl and fnl) and specifically involved in adhesion formation (pai-1 and pai-2) was evaluated.

From the 50 animals that completed the follow-up, 32 animals were identified to have both an ischemic button with an adhesion and an ischemic button that failed to induce adhesion formation. In one sample, the RNA concentration was too low and in one sample no expression was detected for housekeeping genes, making normalisation impossible. Both samples contained an ischemic button with an adhesion and were together with their corresponding control sample excluded from the analysis. Of the 30 animals available for qPCR analysis, 12 were allocated to the HA group, 10 to the HA-CMC group and 8 to the control group.

Clec10a, a M2-associated gene, was 1.36-fold higher expressed in tissue with adhesions compared to tissue without adhesions $(P=0.025)$. Another gene typically expressed in $\mathrm{M} 2, c d 163$, showed a higher expression in presence of adhesions compared to absence of adhesions (1.80-fold, $P=0.003)$ (Figure 7.1). Genes specific for M1 polarisation (il6, nos2, cd86 and infg) did not reach a significant difference between samples with and without adhesions (Figure 7.2). Regarding fibroblast activation, a 5.14-fold higher expression of $\mathrm{mmpl}$ was encountered in samples with adhesion in comparison to samples with no adhesions $(P=0.028)$ (Figure 7.3). Other markers for fibroblast activation (collal and $f n l$ ) showed no differences. Pai-1 and pai-2, known to be involved in adhesion formation, did not differ between groups (Figure 7.4). 
(a)

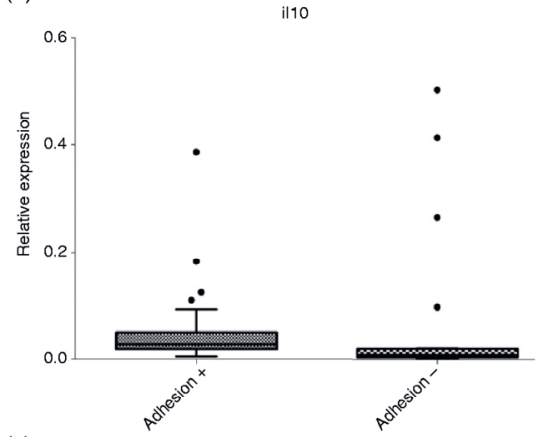

(c)

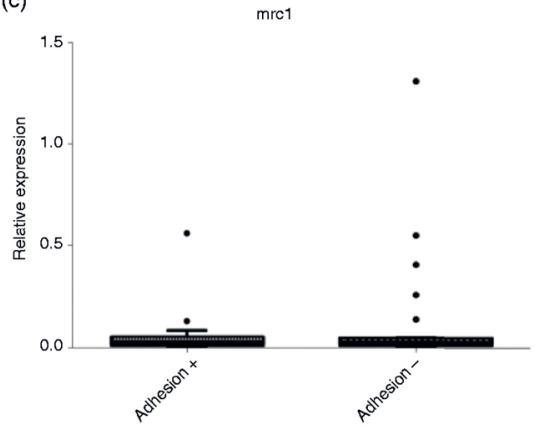

(b)

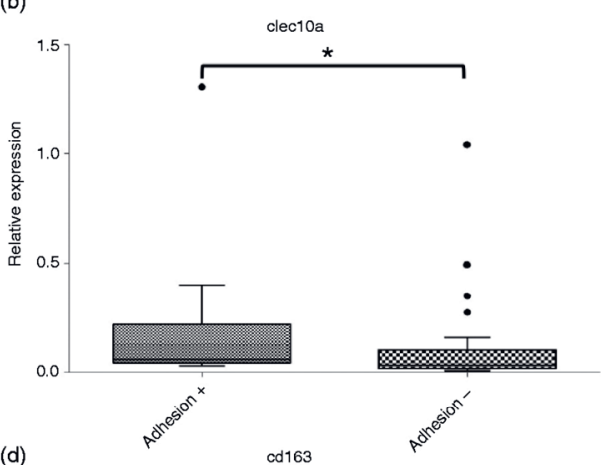

(d)

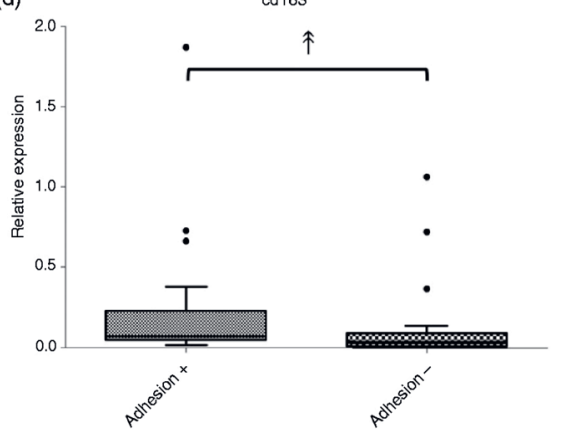

(e)

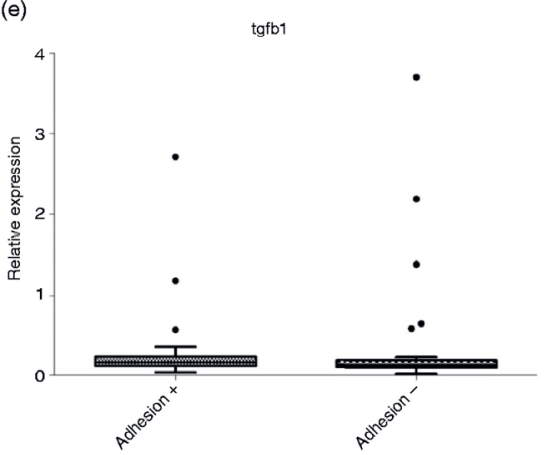

Figure 7.1 Relative expression of genes associated with M2 ((a) il10, (b) clec10a, (c) mrc1, (d) cd163, (e) tgfb1) comparing tissue with adhesions to tissue without adhesions depicting medians with IQR. ${ }^{*} P$-value $=0.025 ;{ }^{*} P$-value $=0.003$. 


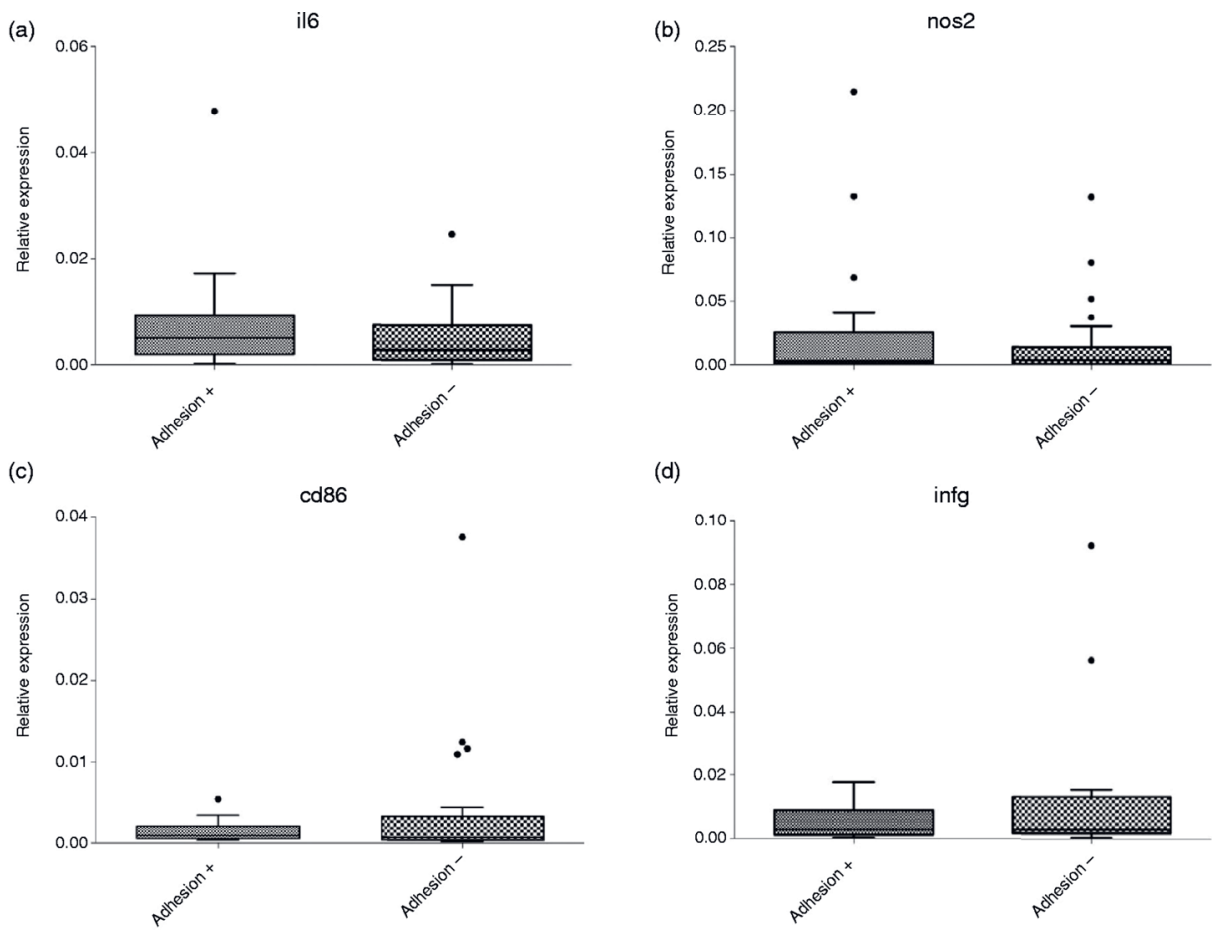

Figure 7.2 Relative expression of genes associated with M1 ((a) il6, (b) nos2, (c) cd86, (d) infg) comparing tissue with adhesions to tissue without adhesions depicting medians with IQR. 
(a)

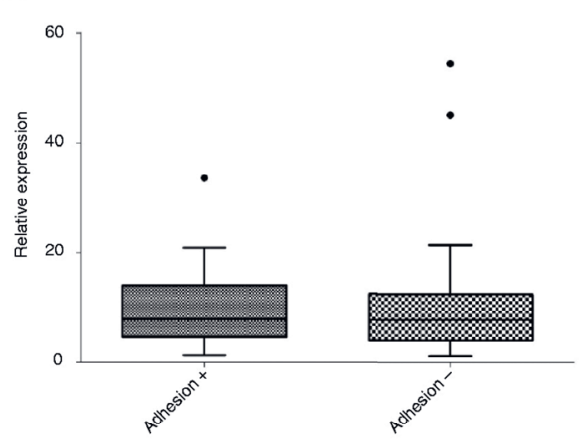

(c)

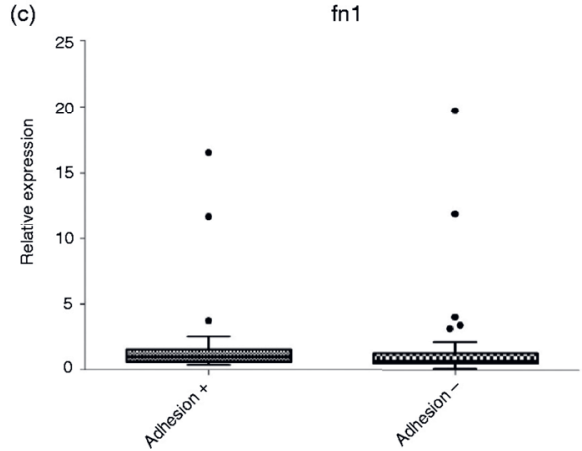

(b) $\mathrm{mmp1}$

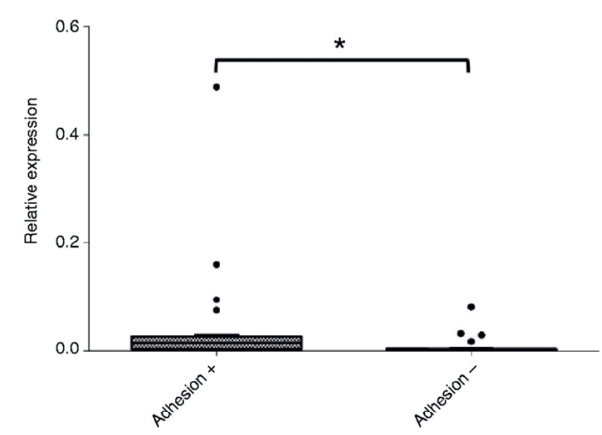

fn1

Figure 7.3 Relative expression of genes associated with fibroblast activation ((a) col1a1, (b) mmp1, (c) $\mathrm{fn} 1$ ) comparing tissue with adhesions to tissue without adhesions depicting medians with IQR. $* P$-value $=0.028$. 

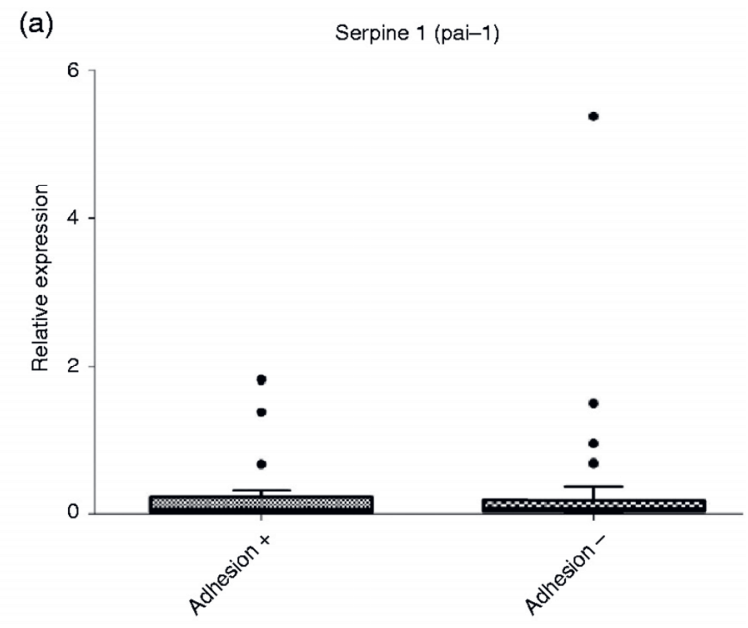

(b)

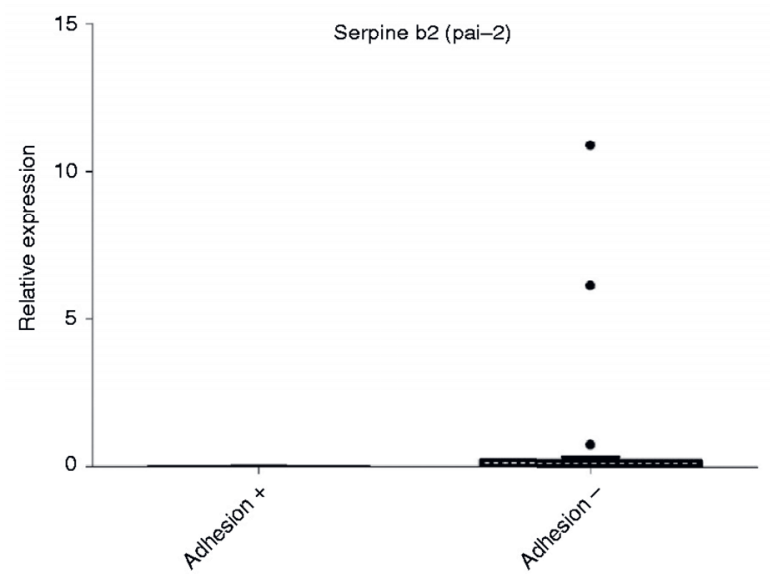

Figure 7.4 Relative expression of genes associated with adhesions formation ((a) Serpine1, (b) Serpineb2) comparing tissue with adhesions to tissue without adhesions depicting medians with IQR.

\section{Discussion}

Intra-abdominal formation of adhesions continues to be a burdening complication for patients who have had surgery., ${ }^{3,4}$ Preventive measures with satisfying results are not available. $^{7,8} \mathrm{HA}$ is proposed in this experiment for the prevention of adhesion formation and has the same main component as HA-CMC, hyaluronic acid. The HA 
gel was crosslinked and differed from HA-CMC by its viscous properties. Due to these viscous properties, it can be used during laparoscopic procedures in contrast to antiadhesive barrier sheets, such as HA-CMC. Instead of an antiadhesive sheet (HA$\mathrm{CMC}$ ) that only provides a barrier between the intra-abdominal organs and the incision in the abdominal wall, HA spreads throughout the abdominal cavity acting as a barrier.

In this study, no reduction in macroscopic adhesion formation was established by the application of HA gel compared to HA-CMC and the control group. A significant lower organ involvement in adhesion formation was found in the HA group (median 2.0, IQR 0.0) compared to the HA-CMC (median 2.0, IQR 1.0) and control group (median 2.0, IQR 1.0). Furthermore, a higher gene expression of clec10a and cd163 was encountered, both typical markers for M2, so an association between adhesion formation and M2 expression was concluded.

Regarding the lower organ involvement after application of HA gel, interestingly the effect was most profound when evaluating organ involvement without the greater omentum and scrotal fat. However, this subgroup analysis did not reach significance. This result emphasizes the difficulty in preventing adhesions to viscera which move relatively free in the abdominal cavity, such as the greater omentum and scrotal fat.

The Nair and tenacity score were unable to distinct in extent and severity of adhesion formation. The number of buttons involved appears to be a more adequate measure for evaluating the extent of adhesion formation. The Nair score ranges from $0-4$, with grade 3 accounting for multiple adhesions formed. The number of buttons involved in adhesions measures all the individual adhesions formed, which constitutes a more detailed measure of adhesion formation. In this experiment no significant differences were found between the groups. An expert panel evaluated the extent and severity of the adhesions in order to bridge the translational gap between animal studies and clinical practice, which unfortunately did not add new insights in distinction in adhesion formation between HA and the other two groups.

In order to gain more insight in the pathophysiological process of adhesion development and the role of macrophage polarization in adhesion formation, gene expression analysis was performed to compare buttons with adhesions and buttons without adhesions within the same animal and irrespective of treatment group. Macrophages play a pivotal role in the pathophysiologic process of adhesion formation; it has been shown that depletion of macrophages results in lower adhesion scores. ${ }^{14}$ Additionally, the macrophage M1/M2 paradigm was identified, describing a continuum between two functionally polarized states of activated macrophages. On one side M1 is known as the classical or pro-inflammatory macrophage associated with tissue injury 
and $\mathrm{M} 2$ on the other side of the spectrum has more anti-inflammatory properties, such as extracellular matrix remodeling and fibroblast regulation. ${ }^{16-18}$

In this study, we demonstrated that buttons with adhesions showed significantly more M2-related gene expression then buttons without adhesion formation. This is based on a pattern of significantly higher expression of clec10a and $c d 163$ (two markers typical for M2) in buttons with adhesions. Genes specific for M1 did not show any significant expression differences. The role of macrophage polarization has been described earlier in a mouse model, but the opposite effect was observed after seven days of follow-up in an ischemic button model in mice. Higher expression of M2 markers were encountered in tissue without adhesion formation, suggesting a protective effect from M2 polarization. ${ }^{30}$ This contradicts the known function of M2, which is extra-cellular matrix remodeling and fibroblast regulation. ${ }^{16-18}$ In our experiment, adhesion formation is associated with increased M2 polarization. Subsequently, it is agreed that mesothelial regeneration takes place 5 to 8 days post injury, ${ }^{31}$ the question remains why after 14 days still a high M2 expression is encountered in buttons with adhesions. This could be explained by ongoing tissue remodeling, but could also be a prolonged activation of M2 macrophages as part of the pathophysiologic process of adhesion formation.

Subsequently, a significantly higher mmpl expression was found in buttons with adhesions compared to buttons without adhesions. This is consistent with findings from in-vitro studies, where fibroblasts from adhesions expressed higher mmpl levels compared to normal peritoneum fibroblasts. ${ }^{15}$ The high expression of $\mathrm{mmpl}$ in adhesive tissue is linked to continued, prolonged tissue remodeling in adhesions after development. Also wound healing of the peritoneum is altered by unregulated production of $m m p 1 .{ }^{13}$

The inhibition of the fibrinolytic system is thought to play an important role in the pathophysiology of adhesion formation. High levels of pai-1 and pai-2 are associated with a decreased fibrinolytic activity and are therefore associated with adhesion formation. ${ }^{12}$ In this experiment, although pai-1 is a stronger inhibitor than pai-2, both were analyzed as specific markers for adhesion formation. ${ }^{12,32}$ No differences in pai- 1 or pai-2 expression between tissue with and without adhesions were found. In animal studies, pai- 1 levels peaked 1 day after surgery, ${ }^{14}$ but in human studies also differences were found years after the first operation, during reoperation. ${ }^{33}$ However, in these studies adhesive tissue was compared to normal peritoneum. ${ }^{14,33}$ It appears that pai-1 and pai-2 influence adhesion formation by inter-acting with the fibrinolytic system in the acute phase and early postoperative period, which may account for the absence of expression after 14 days in our experiment.

The animals in this experiment act as their own control by taking two tissue samples, one ischemic button with adhesions and one without adhesions. The differences that 
were found between both conditions suggest polarization towards M2 and higher fibroblast activation in adhesional tissue and suggest that adhesion formation is a rather local process. In this experiment the dynamic process of macrophage polarization is investigated at one time-point. The exact pathophysiologic process of adhesion formation and the role of macrophage polarization are not clear yet. The focus should be on multiple time-points to evaluate the course in time and on the interaction between the fibrinolytic system, macrophage polarization and fibroblast activation.

Some limitations of this animal experiment need to be discussed. Starting with the impossibility of blinding due to the obvious difference of the investigated materials, which could be a possible source of bias. However, the animals were randomly allocated to a group and equally divided over the cages to minimize the occurrence of possible bias. Data processing and analysis was performed blind. Subsequently, an evaluation of photographs of the adhesions by three experts was performed blind. In this study a gel barrier is com-pared to a sheet barrier, which might perform their barrier function differently. However, is the HA-CMC barrier frequently used in clinical practice and most extensively researched, which makes it the obvious candidate for comparison. The Nair and tenacity score appear to be inaccurate for adhesion assessment in the ischemic button model, for this model induces a great deal of adhesions throughout the abdomen. The number of buttons appears to be more suitable in this case.

\section{Conclusion}

HA gel application reduces the number of organs involved in adhesion formation in an ischemic button model, but shows no significant overall reduction in the formation of adhesions. Macrophage polarization towards M2 and high mmpl expression are associated with adhesion formation. 


\section{References}

1. Menzies D, Ellis H. Intestinal obstruction from adhesions--how big is the problem? Ann R Coll Surg Engl. 1990;72(1):60-3.

2. Stommel MWJ, Ten Broek RPG, Strik C, Slooter GD, Verhoef C, Grunhagen DJ, et al. Multicenter Observational Study of Adhesion Formation After Open-and Laparoscopic Surgery for Colorectal Cancer. Ann Surg. 2018;267(4):743-8.

3. Parker MC, Ellis H, Moran BJ, Thompson JN, Wilson MS, Menzies D, et al. Postoperative adhesions: ten-year follow-up of 12,584 patients undergoing lower abdominal surgery. Dis Colon Rectum. 2001;44(6):822-29; discussion 9-30.

4. Parker MC, Wilson MS, Menzies D, Sunderland G, Clark DN, Knight AD, et al. The SCAR-3 study: 5 -year adhesion-related readmission risk following lower abdominal surgical procedures. Colorectal Dis. 2005;7(6):551-8.

5. ten Broek RP, Issa Y, van Santbrink EJ, Bouvy ND, Kruitwagen RF, Jeekel J, et al. Burden of adhesions in abdominal and pelvic surgery: systematic review and met-analysis. BMJ. 2013;347:f5588.

6. ten Broek RP, Strik C, Issa Y, Bleichrodt RP, van Goor H. Adhesiolysis-related morbidity in abdominal surgery. Ann Surg. 2013;258(1):98-106.

7. Kumar S, Wong PF, Leaper DJ. Intra-peritoneal prophylactic agents for preventing adhesions and adhesive intestinal obstruction after non-gynaecological abdominal surgery. Cochrane Database Syst Rev. 2009(1):CD005080.

8. Ten Broek RPG, Stommel MWJ, Strik C, van Laarhoven C, Keus F, van Goor H. Benefits and harms of adhesion barriers for abdominal surgery: a systematic review and meta-analysis. Lancet. 2014;383(9911):48-59.

9. van Steensel S, van den Hil LCL, Schreinemacher MHF, Ten Broek RPG, van Goor H, Bouvy ND. Adhesion awareness in 2016: An update of the national survey of surgeons. PLoS One. 2018;13(8):e0202418.

10. Beck DE, Cohen Z, Fleshman JW, Kaufman HS, van Goor H, Wolff BG, et al. A prospective, randomized, multicenter, controlled study of the safety of Seprafilm adhesion barrier in abdominopelvic surgery of the intestine. Dis Colon Rectum. 2003;46(10):1310-9.

11. Maciver AH, McCall M, James Shapiro AM. Intra-abdominal adhesions: cellular mechanisms and strategies for prevention. Int J Surg. 2011;9(8):589-94.

12. Arung W, Meurisse M, Detry O. Pathophysiology and prevention of postoperative peritoneal adhesions. World J Gastroenterol. 2011;17(41):4545-53.

13. Chegini N, Kotseos K, Zhao Y, Ma C, McLean F, Diamond MP, et al. Expression of matrix metalloproteinase (MMP-1) and tissue inhibitor of MMP in serosal tissue of intraperitoneal organs and adhesions. Fertil Steril. 2001;76(6):1212-9.

14. Honjo K, Munakata S, Tashiro Y, Salama Y, Shimazu H, Eiamboonsert S, et al. Plasminogen activator inhibitor-1 regulates macrophage-dependent postoperative adhesion by enhancing EGF-HER1 signaling in mice. FASEB J. 2017;31(6):2625-37.

15. Saed GM, Kruger, M. \& Diamond, M.P. . Enhanced matrix metalloproteinase expression by Tisseel in mesothelial cells, normal peritoneal fibroblasts, and adhesion fibroblasts. . Eur J Plast Surg (2006) 2006(28):472-9

16. Martin P, Leibovich SJ. Inflammatory cells during wound repair: the good, the bad and the ugly. Trends Cell Biol. 2005;15(11):599-607.

17. Wynn TA, Barron L. Macrophages: master regulators of inflammation and fibrosis. Semin Liver Dis. 2010;30(3):245-57.

18. Martinez FO, Gordon S. The M1 and M2 paradigm of macrophage activation: time for reassessment. F1000Prime Rep. 2014;6:13.

19. Liu C, Lu Q, Zhang Z, Xue M, Zhang Y, Zhang Y, et al. A Randomized Controlled Trial on the Efficacy and Safety of a New Crosslinked Hyaluronan Gel in Reducing Adhesions after Gynecologic Laparoscopic Surgeries. J Minim Invasive Gynecol. 2015;22(5):853-63. 
20. Kraemer B, Wallwiener C, Rajab TK, Brochhausen C, Wallwiener M, Rothmund R. Standardised models for inducing experimental peritoneal adhesions in female rats. Biomed Res Int. 2014;2014:435056.

21. Rajab TK, Wauschkuhn CA, Smaxwil L, Kraemer B, Wallwiener M, Wallwiener CW. An improved model for the induction of experimental adhesions. J Invest Surg. 2010;23(1):35-9.

22. Whang SH, Astudillo JA, Sporn E, Bachman SL, Miedema BW, Davis W, et al. In search of the best peritoneal adhesion model: comparison of different techniques in a rat model. $\mathrm{J}$ Surg Res. 2011;167(2):245-50.

23. Nair SK, Bhat IK, Aurora AL. Role of proteolytic enzyme in the prevention of postoperative intraperitoneal adhesions. Arch Surg. 1974;108(6):849-53.

24. Zuhlke HV, Lorenz EM, Straub EM, Savvas V. [Pathophysiology and classification of adhesions]. Langenbecks Arch Chir Suppl II Verh Dtsch Ges Chir. 1990:1009-16.

25. Lucas PA, Warejcka DJ, Young HE, Lee BY. Formation of abdominal adhesions is inhibited by antibodies to transforming growth factor-beta1. J Surg Res. 1996;65(2):135-8.

26. Peyton CC, Keys T, Tomblyn S, Burmeister D, Beumer JH, Holleran JL, et al. Halofuginone infused keratin hydrogel attenuates adhesions in a rodent cecal abrasion model. J Surg Res. 2012;178(2): 545-52.

27. Schreinemacher MH, Emans PJ, Gijbels MJ, Greve JW, Beets GL, Bouvy ND. Degradation of mesh coatings and intraperitoneal adhesion formation in an experimental model. Br J Surg. 2009;96(3): 305-13.

28. van den Hil LCL, Vogels RRM, van Barneveld KWY, Gijbels MJJ, Peutz-Kootstra CJ, Cleutjens JPM, et al. Comparability of histological outcomes in rats and humans in a hernia model. J Surg Res. 2018;229:271-6.

29. Ye J, Coulouris G, Zaretskaya I, Cutcutache I, Rozen S, Madden TL. Primer-BLAST: a tool to design target-specific primers for polymerase chain reaction. BMC Bioinformatics. 2012;13:134.

30. Hong GS, Schwandt T, Stein K, Schneiker B, Kummer MP, Heneka MT, et al. Effects of macrophagedependent peroxisome proliferator-activated receptor gamma signalling on adhesion formation after abdominal surgery in an experimental model. Br J Surg. 2015;102(12):1506-16.

31. diZerega GS, Campeau JD. Peritoneal repair and post-surgical adhesion formation. Hum Reprod Update. 2001;7(6):547-55.

32. Ivarsson ML, Bergstrom M, Eriksson E, Risberg B, Holmdahl L. Tissue markers as predictors of postoperative adhesions. Br J Surg. 1998;85(11):1549-54.

33. Holmdahl L, Kotseos K, Bergstrom M, Falk P, Ivarsson ML, Chegini N. Overproduction of transforming growth factor-betal (TGF-beta1) is associated with adhesion formation and peritoneal fibrinolytic impairment. Surgery. 2001;129(5):626-32. 


\section{Chapter 8}

\section{Comparison of coated meshes for intraperitoneal placement in animal studies, a systematic review and meta-analysis}

Hong Liu, Sebastiaan van Steensel, Maurits-Jan Gielen, Timon Vercoulen, Jarno Melenhorst, Bjorn Winkens, Nicole D. Bouvy 


\section{Abstract}

\section{Purpose}

Laparoscopic intraperitoneal onlay mesh in hernia repair can result in adhesions leading to intestinal obstruction and fistulation. The aim of this systematic review is to compare the effects of mesh coatings reducing the tissue-to-mesh adhesion in animal studies.

\section{Methods}

Pubmed and Embase were systematically searched. Animal experiments comparing intraperitoneally placed meshes with coatings were eligible for inclusion. Only studies with comparable follow-up, measurements, and species were included for data pooling and subsequent meta-analysis.

\section{Results}

A total of 131 articles met inclusion criteria, with 4 studies integrated into one comparison and 5 studies integrated into another comparison. Compared to uncoated polypropylene (PP) mesh, PP mesh coated with hyaluronic acid/carboxymethyl cellulose (HA/CMC) showed significantly reduced adhesion formation at follow-up of 4 weeks measured with adhesion score of extent (random effects model, mean difference, $-0.96,95 \% \mathrm{CI}-1.32$ to $-0.61, P<0.001, \mathrm{I}^{2}=23 \%$; fixed effects model, mean difference, $-0.94,95 \%$ CI -1.25 to $-0.63, P<0.001, \mathrm{I}^{2}=23 \%$ ). Compared to PP mesh, polyester mesh coated with collagen ( $\mathrm{PC}$ mesh) showed no significant difference at follow-up of 4 weeks regarding percentage of adhesion-area on a mesh, using random effects model (mean difference $-11.69,95 \%$ CI -44.14 to $20.76, P=0.48, \mathrm{I}^{2}=92 \%$ ). However, this result differed using fixed effects model (mean difference $-25.55,95 \%$ CI -33.70 to $-7.40, P<0.001, \mathrm{I}^{2}=92 \%$ ).

\section{Conclusion}

$\mathrm{HA} / \mathrm{CMC}$ coating reduces adhesion formation to PP mesh effectively at a follow-up of 4 weeks, while the antiadhesive properties of PC mesh are inclusive comparing all study data. 


\section{Introduction}

Incisional hernia ( $\mathrm{IH})$ is one of the most common complications after laparotomy, with an incidence of around $13 \%$ after two years. ${ }^{1}$ Generally, IH is defined as a gap or fascia defect, in the area of abdominal wall scars, that can be detected by clinical examination or imaging. ${ }^{2}$ The number of patients having undergone IH repair was estimated to 300,000 in Europe in 2006., ${ }^{3,4}$ Laparoscopic intraperitoneal onlay mesh placement (IPOM) is clinically available for hernia repair, usually indicated in patients with high risk of infection (diabetes, obesity, compromised immunity), recurrent hernia after open repair, Swiss-cheese hernia (multiple small defects), and lateral hernia (L1-L3) defects. ${ }^{5}$ Meshes in IPOM position may result in adhesions leading to chronic pain, ${ }^{6}$ intestinal obstruction, ${ }^{7}$ difficulties at reoperation, ${ }^{8}$ and even fistulation. ${ }^{9,10}$ Consequently, protective layers were developed to coat the mesh in order to prevent adhesion formation.

Animal experiments are usually performed to test antiadhesion effects of coatings applied to meshes, prior to potential translation to humans. ${ }^{11}$ Although a large number of animal experiments have been conducted for this reason, systematic reviews of these comparisons are scarce. ${ }^{12}$ It is a challenge to identify and compare meshes with identical conditions, i.e. identical species, follow-ups, measurements, and available data type. However, the number of animal experiments exploring an optimal coated mesh is increasing. A thorough overview of coatings to prevent adhesion formation on meshes is essential to the design of prospective animal experiments. The aim of this systematic review is to compare the effects of mesh coatings reducing the tissue-tomesh adhesion in animal studies.

\section{Methods}

This meta-analysis was performed according to the SYRCLE guidelines and registered at PROSPERO [nr: CRD42018089892].

\section{Inclusion and exclusion criteria}

All animal studies investigating $\mathrm{IH}$ repair, comparing tissue-to-mesh adhesion intraperitoneally between a non-coated mesh and a coated mesh, or between coated meshes, were eligible for inclusion. Studies with identical type of two meshes (the same mesh material with the same coating material), comparable follow-up, identical measurements, and species were integrated in this meta-analysis. 
Studies that only compared non-coated meshes or did not report data regarding tissueto-mesh adhesions were excluded. Additionally, human trials, in vitro, and ex vivo experiments were excluded. There were no restrictions regarding species of animals, age, weight, and gender. Articles had to be written in English to be included. Studies were not excluded based on publication date.

\section{Search strategies}

Pubmed and Embase were systematically searched on the $22^{\text {nd }}$ of January, 2019. MeSH terms combined with free-text terms regarding IH repair, intraperitoneal mesh placement, and adhesion formation were used to search these databases. The full search strategies are available in Supplementary Table S8.1. The search was designed with the help of an experienced librarian from Maastricht University.

\section{Study selection}

The search results were imported into a citation manager (EndNote ${ }^{\mathrm{TM}} \mathrm{X} 7$, Clarivate Analytics). Duplicates were removed. After title and abstract screening, full-text screening was conducted by two independent researchers (MJ and TM) to identify the included articles. Disagreement was resolved by discussion and if needed a third researcher (HL) was contacted for arbitration.

\section{Data extraction}

Data extraction was performed using a standard form, which included general study characteristics (the first author and the year of publication), animal characteristics (the animal species, the design of animal experiments, the animal model used, and the follow-up), mesh characteristics (types of the mesh, material and structures of the mesh, location of the placement, and fixation of the mesh), outcomes (extent score, tenacity score, adhesion-area, percentage of adhesion-area on a mesh, and adhesion incidence).

\section{Quality assessment}

The quality of integrated studies were assessed by two independent researchers (HL and MJ), using the SYRCLE's risk of bias tool. ${ }^{13}$ This tool is an adapted version of the Cochrane risk of bias tool and specially developed for assessing the quality of animal studies. Briefly, this tool comprises 10 items including the assessment of selection bias, performance bias, detection bias, attrition bias, reporting bias, and other bias. 


\section{Data synthesis and statistical analysis}

The meta-analysis was conducted using Review Manager (RevMan) [Computer program], Version 5.3 (Copenhagen: The Nordic Cochrane Centre, The Cochrane Collaboration, 2014). The identical comparisons of tissue-to-mesh adhesion at the same follow-up with the same species were pooled separately. In the comparison between polypropylene (PP) mesh and hyaluronic acid/carboxymethyl cellulose (HA/CMC) coated mesh, data of adhesion extent score were summarized to mean \pm standard deviation if there were details reporting the extent score of adhesion or percentage of adhesion-area on mesh in every animal. The extent score of adhesion used the following score: grade $0,0 \%$; grade $1,1-25 \%$; grade $2,26-50 \%$; grade $3,51-75 \%$; and grade $4,>75 \%$ of mesh surface. ${ }^{14}$ In the comparison between PP mesh and PC mesh, data of percentage of adhesion-area on mesh, presented by median, range and interquartile range, were conversed to mean \pm standard deviation by formulas introduced by Wan X et al. ${ }^{15}$ and principles in Corhance handbook. ${ }^{16}$ Percentage of adhesion-area was expressed as $100 \% \times$ adhesion-area on the mesh/the mesh-area in the evaluation day. Treatment effects of data were expressed as mean difference with $95 \%$ confidence intervals and the inverse variance method was used. Both fixed effects model and random effects model were applied and $\mathrm{I}^{2}$ was used to express heterogeneity. If the pooled estimates are comparable between the fixed effects model and the random effects model, the results of the fixed effects model are preferred for the data integration. Otherwise, the results of the random effects model are preferred due to the obvious heterogeneity indicated by the results of two effects models. ${ }^{17}$

\section{Results}

A total of 705 articles were acquired from the search on Pubmed and Embase after removing the duplicates. The flow diagram (Figure 8.1) displays the number of included, excluded and analysed articles. Finally 131 articles met the inclusion criteria. The comparisons of meshes usually applied in clinical practice were attached in Supplementary Tables S8.2-S8.12, containing 68 articles. The other 63 articles were not summarized because an identical comparison of two meshes of different types was barely found in these articles. Four articles were pooled into meta-analysis for the comparison of PP mesh and HA/CMC mesh, ${ }^{18-21}$ and five articles for the comparison of PP mesh and PC mesh. ${ }^{22-26}$ 


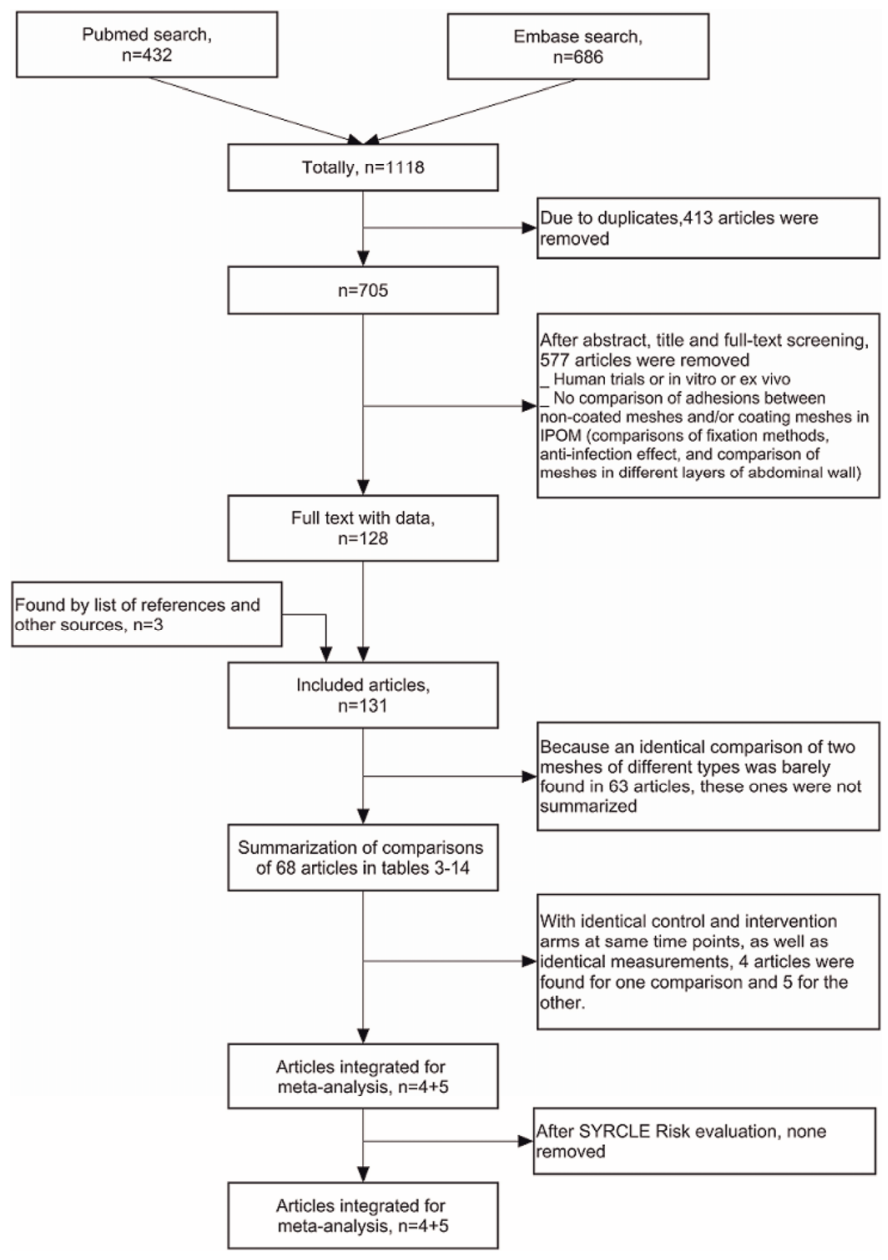

Figure 8.1 Flow diagram.

\section{Outcome of meta-analysis}

\section{Risk evaluation}

All nine articles neither mentioned how random sequences were generated nor how allocation of animals was concealed ${ }^{18-26}$ (Figures 8.2-8.3). Two out of these nine articles mentioned blinding of both performance and detection, ${ }^{20,23}$ while two other articles mentioned only blinding of detection. ${ }^{24,25}$ One article was found with incomplete data, not mentioning the expression of data type, ${ }^{26}$ which implies that mean \pm standard deviation or mean \pm standard error cannot be identified directly from the 
article. One article was considered to show selective reporting ${ }^{25}$ as animals without adhesion were not mentioned in their results.

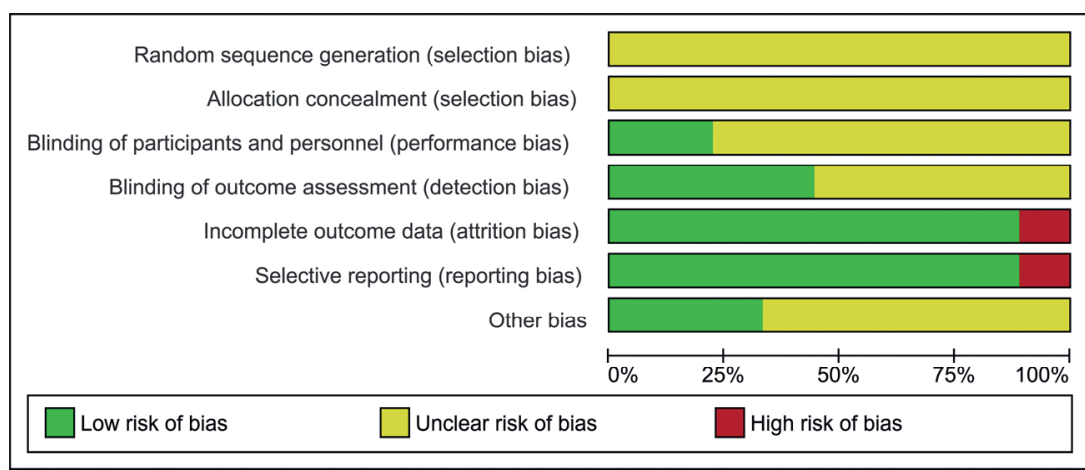

Figure 8.2 Risk of bias graph presented as a percentage of all included studies.

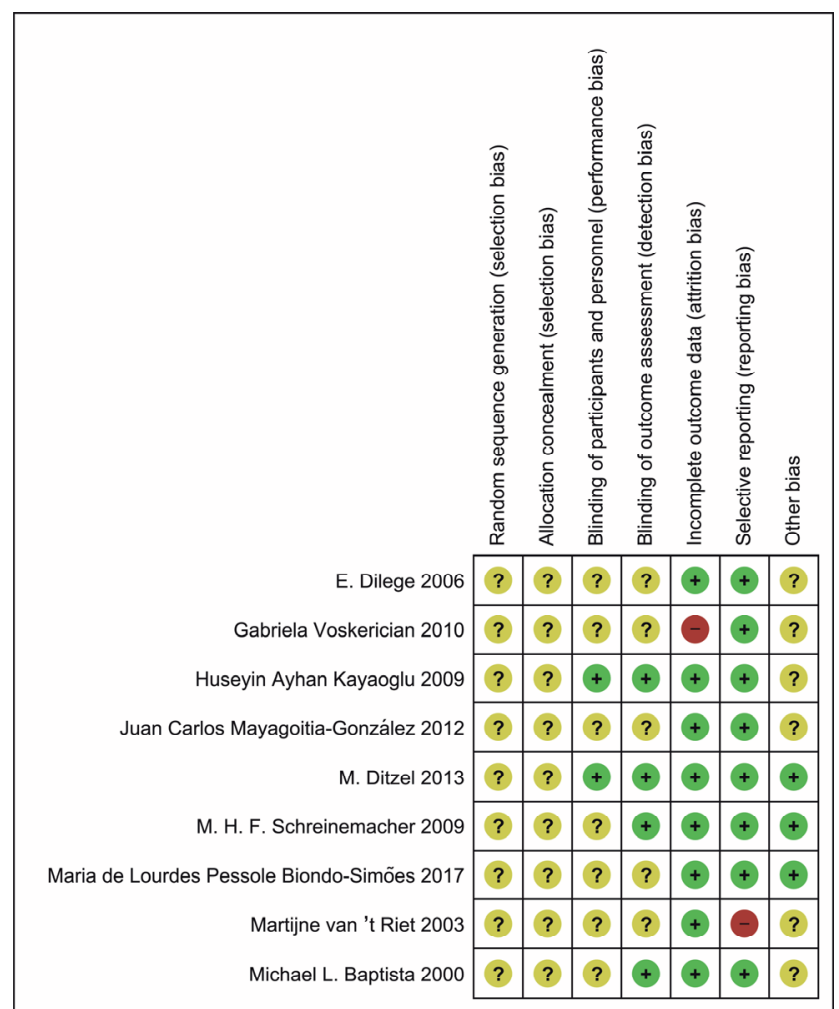

Figure 8.3 Risk of bias summary based on the judgement of reviews. 
Extent score of adhesion, PP mesh VS HA/CMC coated PP mesh

Compared to PP mesh at a follow-up of four weeks in rats, HA/CMC coated PP mesh demonstrated a significantly lower mean adhesion formation measured with extent score of adhesion (random effects model, mean difference, $-0.96,95 \% \mathrm{CI}-1.32$ to -0.61 , $P<0.001, \mathrm{I}^{2}=23 \%$; fixed effects model, mean difference, $-0.94,95 \%$ CI -1.25 to -0.63 , $P<0.001, \mathrm{I}^{2}=23 \%$ ). The results of the random effects and the fixed effects model in this comparison were similar, but the result of the fixed effects model was more precise due to the narrower confidence interval. Therefore, the result of the fixed effects model shown in Figure 8.4 was preferred. In total, four studies with 118 animals were included in this comparison. The PP mesh in these four studies was Prolene ${ }^{\circledR}$ mesh (a heavy-weight PP mesh with medium pore size fabricated by monofilament), ${ }^{19}$ Marlex ${ }^{\circledR}$ mesh (a heavy-weight PP mesh with medium pore size fabricated by monofilament), ${ }^{18}$ Surgipro ${ }^{\circledR}$ mesh (a heavy-weight PP mesh with medium pore size fabricated by multifilament), ${ }^{20}$ or a heavy polypropylene mesh. ${ }^{21}$ The HA/CMC coated PP mesh was Sepramesh ${ }^{\circledR}$ (PP mesh with HA/CMC coating), ${ }^{19,20}$ PP mesh covered by a HA/CMC membrane, ${ }^{18}$ or PP mesh coated by a HA/CMC gel. ${ }^{21}$ The sutures for fixation of these meshes to abdominal wall were polypropylene sutures in three of four studies ${ }^{18-20}$ and polyglyconate suture in one of four studies. ${ }^{21}$ All these four studies showed HA/CMC coated PP meshes were superior to PP meshes in adhesion reduction measured by extent score of adhesion.

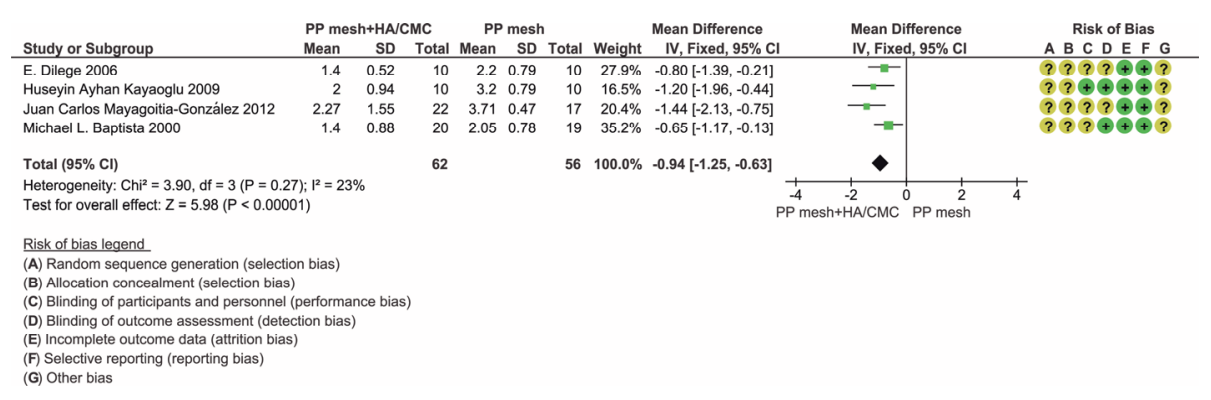

Figure 8.4 Comparison between polypropylene mesh and polypropylene mesh with HA/CMC coating in forest plot at 4 weeks follow-up in rats.

\section{Percentage of adhesion-area, PP mesh VS PC mesh}

Compared to PP mesh at a follow-up of four weeks in rats, PC mesh showed a nonsignificant lower mean percentage of adhesion-area on mesh (random effects model, mean difference $-11.69,95 \%$ CI -44.14 to $20.76, P=0.48, \mathrm{I}^{2}=92 \%$; fixed effects model, mean difference $-25.55,95 \%$ CI -33.70 to $-7.40, P<0.001, \mathrm{I}^{2}=92 \%$ ). The result of the random effects model shown in Figure 8.5 was preferred for the integration but the obvious heterogeneity was indicated by the results of two effects models. A total of 
five studies with 97 animals were included in this comparison. The PP mesh in four of five studies was Prolene ${ }^{\circledR}$ mesh, ${ }^{23-26}$ while Marlex ${ }^{\circledR}$ was used in the other study. ${ }^{22}$ The PC mesh in all of these five studies was Parietex ${ }^{\circledR}$ Composite mesh. All the meshes in these five studies were secured to the abdominal wall with Prolene ${ }^{\circledR}$ sutures. Two of these five studies suggested PC mesh had less adhesion formation than PP mesh, ${ }^{23,24}$ while two of five studies showed no benefit of PC mesh on adhesion reduction ${ }^{22,26}$ and one of five studies found PC mesh had higher adhesion formation than PP mesh. ${ }^{25}$

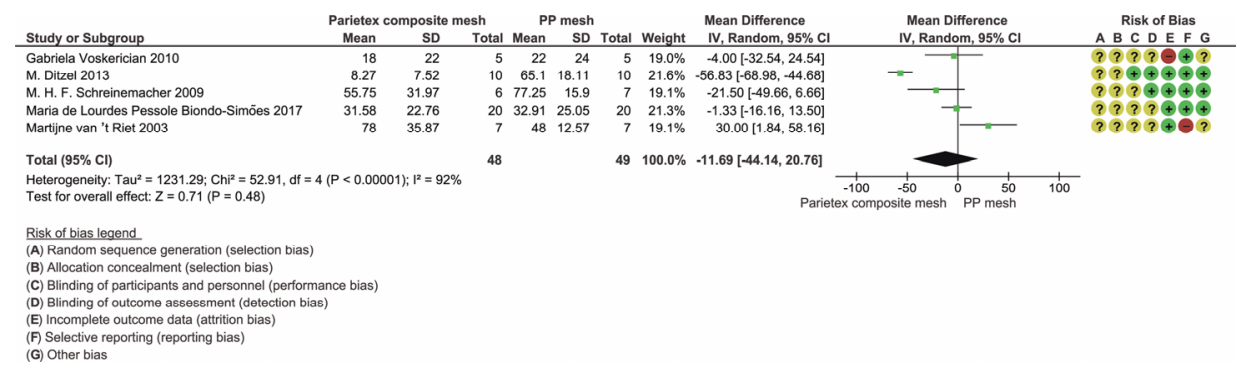

Figure 8.5 Comparison between polypropylene mesh and Parietex Composite mesh in forest plot at 4 weeks follow-up in rats.

\section{Discussion}

Mesh use in IH significantly reduces recurrent IH compared with suture repair alone. However, an ideal mesh for intraperitoneal use remains to be explored due to meshrelated complications. Prosthetic meshes with novel coatings have been developed with high potential to overcome adhesion formation, one of the complications. While numerous animal studies have focused on the antiadhesive effect of coated meshes, a systematic review investigating the optimal antiadhesive mesh has not been conducted before. According to our results, HA/CMC coated meshes and PC meshes were the most common meshes with coatings tested in animal experiments.

Seprafilm ${ }^{\circledR}$ membrane with a component of HA/CMC has been approved by FDA as a barrier for prevention of postoperative adhesion formation since 1996, with the support of several multi-center clinical trials. ${ }^{27,28}$ Due to technical difficulties caused by the adherent membrane used in IPOM, a new composite mesh was developed composed of PP mesh with a HA/CMC coated layer facing the viscera. Most studies supported that HA/CMC coated PP meshes were superior to PP mesh without a coating, although one study demonstrated no preference between both meshes. ${ }^{29}$ Our meta-analysis results showed HA/CMC coated PP meshes significantly reduced the adhesion formation measured by extent score at four weeks. Almost all animal studies included in this 
meta-analysis showed HA/CMC coated PP mesh was preferred to PP mesh alone from one week follow-up to four months follow-up, in rats, rabbits, and pigs. The superiority of HA/CMC coated PP meshes over uncoated PP mesh was found measured by percentage of adhesion-area on mesh, tenacity score of adhesion, extent score of adhesion, number of bowel adhesions to mesh, and rate of adhesion presence. Sasse et al. demonstrated that after HA/CMC coated PP mesh (Sepramesh ${ }^{\circledR}$, Davol, Providence, RI) was implanted intraperitoneally, little to no discomfort related to the mesh was reported in 65 of $72(90.3 \%)$ patients over a follow-up period of 41 months. ${ }^{30}$ Rose et al. provided a case illustrating that less than $25 \%$ of the HA/CMC coated PP mesh (Sepramesh ${ }^{\circledR}$, Genzyme, Biosurgery) was covered by filmy adhesion one year after the placement in patients. ${ }^{31}$ Deeken et al. demonstrated to have initiated a multiinstitutionally clinical trial focusing on the reductive effectiveness of coated meshes against adhesion. $^{12}$

PC mesh is another clinically available mesh with a layer of oxidized type 1 atelocollagen, polyethylene glycol, and glycerol coated on polyester prosthesis. ${ }^{32}$ Some studies suggested it had significant prevention of adhesion formation, measured as percentage of adhesion-area, superior to PP mesh. ${ }^{20,24,29,33-39}$ However, some studies did not find a preference between PC mesh and PP mesh. ${ }^{22,26}$ One study even showed PC mesh presented higher adhesion formation than PP mesh. ${ }^{25}$ Our meta-analysis showed no significant difference to be found between PC mesh and PP mesh in animal experiments using random effects model, noting the heterogeneity of these pooled studies was high. In humans, Chelala et al. reported that, detecting the PC mesh related adhesions in patients with a second look operation, 40 of $85(47.05 \%)$ patients were adhesion-free during a mean of 52-month follow-up using PC mesh, 36 of 85 (42.3\%) were found to have loose adhesions to the omentum, and 9 of $85(10.58 \%)$ patients had mild intestinal adhesions. ${ }^{40}$

To our knowledge, this is the first time a meta-analysis was performed comparing coated meshes in the same conditions in animals. Although a large number of studies focused on evaluating tissue-to mesh adhesions, animal studies with an identical comparison of meshes in the same conditions were scarce. This situation resulted from many different factors between groups, including mesh characteristics, measurement system for the adhesions, follow-up, and animal species.

To this moment, more than 70 types of meshes are commercially available. ${ }^{41}$ Characteristics of a mesh, including coating, material absorption, pore size, weight, constitution, knitting structure, and fixation, played a role in the formation of tissue-tomesh adhesions. Animal studies containing an identical comparison of two types of meshes, regarding the absolutely same characteristics of the two meshes, were scarce. 
The present two integrated studies only focused on the characteristics with the same mesh material and the same coating material.

The measurements of adhesion were generally classified based on percentage of adhesions covering a mesh, adhesion-area, incidence of adhesions, number of adhesions, type of adhesions, density of adhesions, and location of adhesions. No standard scoring system was widely accepted and applied in animal studies. Furthermore, even when aforementioned items were identical, grading of scores varies between studies, ranging from two levels to five levels. A consensus for researchers on a standard evaluation system of adhesion-to-mesh is urgently required.

Follow-up durations for evaluation of adhesion formation vary largely, from three days to one year in included studies, mainly due to differing research questions. Most follow-ups were set at four weeks. Generally, after in vivo implantation of biomaterials, multiple processes happen subsequently, including blood-material interaction, provisional matrix formation, acute and chronic inflammation, and finally formation of granulation tissue and fibrous encapsulation at three to four weeks. ${ }^{42}$ Sulaiman et al. found that, in a mice adhesion model, peritoneal adhesion formation was mainly associated with chronic inflammation instead of acute inflammation. ${ }^{43}$ Recommended by an expert consensus performed by our groups (unpublished), a follow-up of at least four weeks is suitable to assess chronic inflammation in animal experiments.

Appropriate animal species and models are necessary for animal studies. Especially since Van den Hil et al. found that histological outcomes were comparable between rats and humans, concerning adhesion formation and foreign body reaction to meshes. ${ }^{44}$

Heterogeneity in meta-analysis represents between-studies variance, caused by different set-ups of the studies. To investigate heterogeneity of any significance, different statistical methods were applied after data integration of the pooled studies. Fixed effect models assume no heterogeneity exists and the variance between studies is fully caused by within-study variance. In contrast, random effects models include the possible effect of heterogeneity in pooled data. ${ }^{45}$ In the current study, heterogeneity was identified in the studies comparing the PC mesh and PP mesh, despite mesh types, follow-up, species, and fixation material being virtually identical. It was not clear which other factors contribute to this heterogeneity. Subgroup meta-analysis detecting the heterogeneous source in the five pooled studies was complicated, due to the lack of details regarding randomization, animal housing conditions, the influence of different surgeons, and the microbiome, as well as unknown factors that might act as a source of the heterogeneity. 
This meta-analysis encountered several limitations. Firstly, due to heterogeneity of all animal studies only several studies could be included in this meta-analysis and the sample size might not be large enough. This is due to the strict inclusion criteria and not enough studies available. The identical conditions for the comparisons were to ensure true integration of data. Secondly, the conversion of data might normally cause a little bias. Since all the data are converted under the same standards for the integration, consulting with the statistician in our university, bias seems minimal. Lastly, due to the different conditions between animals and humans, translation of our results to humans will still require human trials.

\section{Conclusion}

HA/CMC coating reduces adhesion formation to PP mesh effectively at follow-up of four weeks, while the antiadhesive properties of PC mesh are inclusive comparing all study data. A standard adhesion score assessing tissue-to-mesh adhesions is urgently required to reach a consensus for animal experiments. 


\section{References}

1. Bosanquet DC, Ansell J, Abdelrahman T, Cornish J, Harries R, Stimpson A, et al. Systematic Review and Meta-Regression of Factors Affecting Midline Incisional Hernia Rates: Analysis of 14,618 Patients. PloS one. 2015;10(9):e0138745.

2. Sanders DL, Kingsnorth AN. The modern management of incisional hernias. BMJ. 2012;344:e2843.

3. Sauerland S, Walgenbach M, Habermalz B, Seiler CM, Miserez M. Laparoscopic versus open surgical techniques for ventral or incisional hernia repair. Cochrane Database Syst Rev. 2011(3):CD007781.

4. Poulose BK, Shelton J, Phillips S, Moore D, Nealon W, Penson D, et al. Epidemiology and cost of ventral hernia repair: making the case for hernia research. Hernia : the journal of hernias and abdominal wall surgery. 2012;16(2):179-83.

5. Sharma A, Berger D. The current role of laparoscopic IPOM repair in abdominal wall reconstruction. Hernia : the journal of hernias and abdominal wall surgery. 2018;22(5):739-41.

6. Husain M, Sachan PK, Khan S, Lama L, Khan RN. Role of diagnostic laparoscopy in chronic and recurrent abdominal pain. Trop Gastroenterol. 2013;34(3):170-3.

7. Menzies D, Ellis H. Intestinal obstruction from adhesions--how big is the problem? Ann R Coll Surg Engl. 1990;72(1):60-3.

8. ten Broek RP, Schreinemacher MH, Jilesen AP, Bouvy N, Bleichrodt RP, van Goor H. Enterotomy risk in abdominal wall repair: a prospective study. Annals of surgery. 2012;256(2):280-7.

9. Burger JW, Luijendijk RW, Hop WC, Halm JA, Verdaasdonk EG, Jeekel J. Long-term follow-up of a randomized controlled trial of suture versus mesh repair of incisional hernia. Annals of surgery. 2004;240(4):578-83; discussion 83-5.

10. Shubinets V, Carney MJ, Colen DL, Mirzabeigi MN, Weissler JM, Lanni MA, et al. Management of Infected Mesh After Abdominal Hernia Repair: Systematic Review and Single-Institution Experience. Annals of plastic surgery. 2018;80(2):145-53.

11. Hooijmans CR, Ritskes-Hoitinga M. Progress in using systematic reviews of animal studies to improve translational research. PLoS Med. 2013;10(7):e1001482.

12. Deeken CR, Faucher KM, Matthews BD. A review of the composition, characteristics, and effectiveness of barrier mesh prostheses utilized for laparoscopic ventral hernia repair. Surgical endoscopy. 2012;26(2):566-75.

13. Hooijmans CR, Rovers MM, de Vries RB, Leenaars M, Ritskes-Hoitinga M, Langendam MW. SYRCLE's risk of bias tool for animal studies. BMC Med Res Methodol. 2014;14:43.

14. Diamond MP, Linsky CB, Cunningham T, Constantine B, diZerega GS, DeCherney AH. A model for sidewall adhesions in the rabbit: reduction by an absorbable barrier. Microsurgery. 1987;8(4):197-200.

15. Wan X, Wang W, Liu J, Tong T. Estimating the sample mean and standard deviation from the sample size, median, range and/or interquartile range. BMC Med Res Methodol. 2014;14:135.

16. Higgins JPT; Deeks JJ. Chapter 7: Selecting studies and collecting data. In: Higgins JPT, Green S (editors), Cochrane Handbook for Systematic Reviews of Interventions.2008.

17. Ryan R. Cochrane Consumers and Communication Review Group. 'Cochrane Consumers and Communication Group: meta-analysis‘. http://cccrg.cochrane.org. December 2016 (accessed DATE).2016.

18. Baptista ML, Bonsack ME, Delaney JP. Seprafilm reduces adhesions to polypropylene mesh. Surgery. 2000;128(1):86-92.

19. Dilege E, Coskun H, Gunduz B, Sakiz D, Mihmanli M. Prevention of adhesion to prosthetic mesh in incisional ventral hernias: comparison of different barriers in an experimental model. European surgical research Europaische chirurgische Forschung Recherches chirurgicales europeennes. 2006;38(3):358-64.

20. Kayaoglu HA, Ozkan N, Hazinedaroglu SM, Ersoy OF, Erkek AB, Koseoglu RD. Comparison of adhesive properties of five different prosthetic materials used in hernioplasty. Journal of investigative surgery : the official journal of the Academy of Surgical Research. 2005;18(2):89-95. 
21. Mayagoitia-Gonzalez JC, Gudino-Amezcua LM, Rivera-Barragan V, Mellado-Diaz AV, Diaz-Chavez EP. Prevention of intestinal adhesions as a result of intraperitoneal mesh with the addition of hyaluronic acid/carboxymethylcellulose gel. Experimental model in rats. Cir Cir. 2012;80(2):150-6.

22. Biondo-Simoes ML, Carvalho LB, Conceicao LT, Santos KB, Schiel WA, Arantes M, et al. Comparative study of Polypropylene versus Parietex composite(R), Vicryl(R) and Ultrapro(R) meshes, regarding the formation of intraperitoneal adhesions. Acta cirurgica brasileira. 2017;32(2):98-107.

23. Ditzel M, Deerenberg EB, Grotenhuis N, Harlaar JJ, Monkhorst K, Bastiaansen-Jenniskens YM, et al. Biologic meshes are not superior to synthetic meshes in ventral hernia repair: an experimental study with long-term follow-up evaluation. Surgical endoscopy. 2013;27(10):3654-62.

24. Schreinemacher MH, Emans PJ, Gijbels MJ, Greve JW, Beets GL, Bouvy ND. Degradation of mesh coatings and intraperitoneal adhesion formation in an experimental model. The British journal of surgery. 2009;96(3):305-13.

25. van 't Riet M, de Vos van Steenwijk PJ, Bonthuis F, Marquet RL, Steyerberg EW, Jeekel J, et al. Prevention of adhesion to prosthetic mesh: comparison of different barriers using an incisional hernia model. Annals of surgery. 2003;237(1):123-8.

26. Voskerician G, Jin J, White MF, Williams CP, Rosen MJ. Effect of biomaterial design criteria on the performance of surgical meshes for abdominal hernia repair: a pre-clinical evaluation in a chronic rat model. Journal of materials science Materials in medicine. 2010;21(6):1989-95.

27. Becker JM, Dayton MT, Fazio VW, Beck DE, Stryker SJ, Wexner SD, et al. Prevention of postoperative abdominal adhesions by a sodium hyaluronate-based bioresorbable membrane: a prospective, randomized, double-blind multicenter study. Journal of the American College of Surgeons. 1996;183(4):297-306.

28. Beck DE, Cohen Z, Fleshman JW, Kaufman HS, van Goor H, Wolff BG, et al. A prospective, randomized, multicenter, controlled study of the safety of Seprafilm adhesion barrier in abdominopelvic surgery of the intestine. Diseases of the colon and rectum. 2003;46(10):1310-9.

29. Gonzalez R, Rodeheaver GT, Moody DL, Foresman PA, Ramshaw BJ. Resistance to adhesion formation: a comparative study of treated and untreated mesh products placed in the abdominal cavity. Hernia : the journal of hernias and abdominal wall surgery. 2004;8(3):213-9.

30. Sasse KC, Lim DC, Brandt J. Long-term durability and comfort of laparoscopic ventral hernia repair. JSLS : Journal of the Society of Laparoendoscopic Surgeons. 2012;16(3):380-6.

31. Rose J, Jayaraman S, Colquhoun P, Taylor B. Minimal abdominal adhesions after Sepramesh repair of a parastomal hernia. Can J Surg. 2009;52(5):E211-2.

32. Briennon X, Lermite E, Meunier K, Desbois E, Hamy A, Arnaud JP. Surgical treatment of large incisional hernias by intraperitoneal insertion of Parietex(R) composite mesh with an associated aponeurotic graft (280 cases). J Visc Surg. 2011;148(1):54-8.

33. Schreinemacher MH, van Barneveld KW, Dikmans RE, Gijbels MJ, Greve JW, Bouvy ND. Coated meshes for hernia repair provide comparable intraperitoneal adhesion prevention. Surgical endoscopy. 2013;27(11):4202-9.

34. LeBlanc KA, Bellanger D, Rhynes KVt, Baker DG, Stout RW. Tissue attachment strength of prosthetic meshes used in ventral and incisional hernia repair. A study in the New Zealand White rabbit adhesion model. Surgical endoscopy. 2002;16(11):1542-6.

35. Gaertner WB, Bonsack ME, Delaney JP. Visceral adhesions to hernia prostheses. Hernia : the journal of hernias and abdominal wall surgery. 2010;14(4):375-81.

36. Jacob BP, Hogle NJ, Durak E, Kim T, Fowler DL. Tissue ingrowth and bowel adhesion formation in an animal comparative study: polypropylene versus Proceed versus Parietex Composite. Surgical endoscopy. 2007;21(4):629-33.

37. Bellon JM, Garcia-Carranza A, Jurado F, Garcia-Honduvilla N, Carrera-San Martin A, Bujan J. Peritoneal regeneration after implant of a composite prosthesis in the abdominal wall. World journal of surgery. 2001;25(2):147-52.

38. Hu M, Lin X, Huang R, Yang K, Liang Y, Zhang X, et al. Lightweight, Highly Permeable, Biocompatible, and Antiadhesive Composite Meshes for Intraperitoneal Repairs. Macromolecular bioscience. 2018;18(7):e1800067. 
39. Bellon JM, Rodriguez M, Garcia-Honduvilla N, Pascual G, Gil VG, Bujan J. Peritoneal effects of prosthetic meshes used to repair abdominal wall defects: monitoring adhesions by sequential laparoscopy. Journal of Laparoendoscopic and Advanced Surgical Techniques. 2007;17(2):160-6.

40. Chelala E, Debardemaeker Y, Elias B, Charara F, Dessily M, Alle JL. Eighty-five redo surgeries after 733 laparoscopic treatments for ventral and incisional hernia: adhesion and recurrence analysis. Hernia : the journal of hernias and abdominal wall surgery. 2010;14(2):123-9.

41. Baylon K, Rodriguez-Camarillo P, Elias-Zuniga A, Diaz-Elizondo JA, Gilkerson R, Lozano K. Past, Present and Future of Surgical Meshes: A Review. Membranes (Basel). 2017;7(3).

42. Sheikh Z, Brooks PJ, Barzilay O, Fine N, Glogauer M. Macrophages, Foreign Body Giant Cells and Their Response to Implantable Biomaterials. Materials (Basel). 2015;8(9):5671-701.

43. Sulaiman H, Dawson L, Laurent GJ, Bellingan GJ, Herrick SE. Role of plasminogen activators in peritoneal adhesion formation. Biochem Soc Trans. 2002;30(2):126-31.

44. van den Hil LCL, Vogels RRM, van Barneveld KWY, Gijbels MJJ, Peutz-Kootstra CJ, Cleutjens JPM, et al. Comparability of histological outcomes in rats and humans in a hernia model. The Journal of surgical research. 2018;229:271-6.

45. Barili F, Parolari A, Kappetein PA, Freemantle N. Statistical Primer: heterogeneity, random- or fixedeffects model analyses? Interact Cardiovasc Thorac Surg. 2018;27(3):317-21. 


\section{Supplemental materials}

Table S8.1 Search strategy for Pubmed (a) and Embase database (b).

a

\section{Pubmed}

$(((($ Sepracoat[tiab] or sepramesh[tiab] or seprafilm[tiab] or Parietex[tiab] or hyaluronate carboxymethylcellulose[tiab] or Proceed[tiab] or Vypro[tiab] or Gore-tex mesh[tiab] or Dual mesh[tiab] or Composix[tiab] or Dynamesh[tiab] or Ultrapro[tiab] or Ti-mesh[tiab] or C-Qur[tiab])) OR (((Surgical mesh [MeSH terms] OR Mesh [tiab] OR Meshes [tiab] OR prothes*[tw] OR prosthet*[tw]) AND (Coated [tiab] OR Coating [tiab] OR Composite [tiab] OR Intraperitoneal[tiab] OR Intra-peritoneal[tiab] OR Intraabdominal[tiab] OR transabdominal[tiab] OR IPOM[tiab]))))) AND (((Abdominal hernia [MeSH terms] OR Incisional Hernia [MeSH Terms])) OR ((((Hernia [tiab] OR Hernias[tiab] OR Defect[tiab] OR Defects[tiab] OR herniorrhaphy OR hernioplasty))) AND ((Abdominal [tiab] OR Ventral[tiab] OR Incisional[tiab] OR Incisive [tiab] OR Inguinal[tiab] OR Parastomal [tiab] OR Femoral[tiab] OR Umbilical[tiab] OR Epigastric[tiab] OR Spigelian[tiab] OR Postoperative[tiab] OR Laparotomy [tiab] OR Laparotomies[tiab]OR Celiotomy[tiab] OR Celiotomies[tiab] OR Minilaparotomy[tiab] OR Minilaparotomies[tiab]))))) AND (((“animal experimentation”[MeSH Terms] OR “models, animal”[MeSH Terms] OR "invertebrates”[MeSH Terms] OR “Animals”[Mesh:noexp] OR “animal population groups”[MeSH Terms] OR “chordata”[MeSH Terms:noexp] OR “chordata, nonvertebrate"[MeSH Terms] OR "vertebrates"[MeSH Terms:noexp] OR “amphibians"[MeSH Terms] OR "birds"[MeSH Terms] OR “fishes”[MeSH Terms] OR "reptiles”[MeSH Terms] OR “mammals”[MeSH Terms:noexp] OR “primates”[MeSH Terms:noexp] OR "artiodactyla”[MeSH Terms] OR “carnivora”[MeSH Terms] OR “cetacea”[MeSH Terms] OR “chiroptera”[MeSH Terms] OR “elephants"[MeSH Terms] OR "hyraxes"[MeSH Terms] OR “insectivora”[MeSH Terms] OR "lagomorpha"[MeSH Terms] OR "marsupialia"[MeSH Terms] OR "monotremata"[MeSH Terms] OR "perissodactyla"[MeSH Terms] OR "rodentia"[MeSH Terms] OR "scandentia"[MeSH Terms] OR "sirenia”"[MeSH Terms] OR “xenarthra"[MeSH Terms] OR "haplorhini”[MeSH Terms:noexp] OR “strepsirhini”[MeSH Terms] OR “platyrrhini”[MeSH Terms] OR “tarsii”[MeSH Terms] OR “catarrhini”[MeSH Terms:noexp] OR “cercopithecidae”[MeSH Terms] OR "hylobatidae”[MeSH Terms] OR “hominidae”[MeSH Terms:noexp] OR “gorilla gorilla”[MeSH Terms] OR “pan paniscus”[MeSH Terms] OR “pan troglodytes”[MeSH Terms] OR “pongo pygmaeus”[MeSH Terms]) OR ((animals[tiab] OR animal[tiab] OR mice[Tiab] OR mus[Tiab] OR mouse[Tiab] OR murine[Tiab] OR woodmouse[tiab] OR rats[Tiab] OR rat[Tiab] OR murinae[Tiab] OR muridae[Tiab] OR cottonrat[tiab] OR cottonrats[tiab] OR hamster[tiab] OR hamsters[tiab] OR cricetinae[tiab] OR rodentia[Tiab] OR rodent[Tiab] OR rodents[Tiab] OR pigs[Tiab] OR pig[Tiab] OR swine[tiab] OR swines[tiab] OR piglets[tiab] OR piglet[tiab] OR boar[tiab] OR boars[tiab] OR "sus scrofa"[tiab] OR ferrets[tiab] OR ferret[tiab] OR polecat[tiab] OR polecats[tiab] OR "mustela putorius"[tiab] OR “guinea pigs"[Tiab] OR “guinea pig”[Tiab] OR cavia[Tiab] OR callithrix[Tiab] OR marmoset[Tiab] OR marmosets[Tiab] OR cebuella[Tiab] OR hapale[Tiab] OR octodon[Tiab] OR chinchilla[Tiab] OR chinchillas[Tiab] OR gerbillinae[Tiab] OR gerbil[Tiab] OR gerbils[Tiab] OR jird[Tiab] OR jirds[Tiab] OR merione[Tiab] OR meriones[Tiab] OR rabbits[Tiab] OR rabbit[Tiab] OR hares[Tiab] OR hare[Tiab] OR diptera[Tiab] OR flies[Tiab] OR fly[Tiab] OR dipteral[Tiab] OR drosophila[Tiab] OR drosophilidae[Tiab] OR cats[Tiab] OR cat[Tiab] OR carus[Tiab] OR felis[Tiab] OR nematoda[Tiab] OR nematode[Tiab] OR nematodes[Tiab] OR sipunculida[Tiab] OR dogs[Tiab] OR dog[Tiab] OR canine[Tiab] OR canines[Tiab] OR canis[Tiab] OR sheep[Tiab] OR sheeps[Tiab] OR mouflon[Tiab] OR mouflons[Tiab] OR ovis[Tiab] OR goats[Tiab] OR goat[Tiab] OR capra[Tiab] OR capras[Tiab] OR rupicapra[Tiab] OR chamois[Tiab] OR haplorhini[Tiab] OR monkey[Tiab] OR monkeys[Tiab] OR anthropoidea[Tiab] OR anthropoids[Tiab] OR saguinus[Tiab] OR tamarin[Tiab] OR tamarins[Tiab] OR leontopithecus[Tiab] OR hominidae[Tiab] OR ape[Tiab] OR apes[Tiab] OR "pan paniscus"[Tiab] OR bonobo[Tiab] OR bonobos[Tiab] OR "pan troglodytes"[Tiab] OR gibbon[Tiab] OR gibbons[Tiab] OR siamang[Tiab] OR siamangs[Tiab] OR nomascus[Tiab] OR symphalangus[Tiab] OR chimpanzee[Tiab] OR chimpanzees[Tiab] OR prosimian[Tiab] OR prosimians[Tiab] OR "bush baby"[Tiab] OR bush babies[Tiab] OR galagos[Tiab] OR galago[Tiab] OR pongidae[Tiab] OR gorilla[Tiab] OR gorillas[Tiab] OR “pongo pygmaeus”[Tiab] OR 
orangutan[Tiab] OR orangutans[Tiab] OR lemur[Tiab] OR lemurs[Tiab] OR lemuridae[Tiab] OR horse[Tiab] OR horses[Tiab] OR equus[Tiab] OR cow[Tiab] OR calf[Tiab] OR bull[Tiab] OR chicken[Tiab] OR chickens[Tiab] OR gallus[Tiab] OR quail[Tiab] OR bird[Tiab] OR birds[Tiab] OR quails[Tiab] OR poultry[Tiab] OR poultries[Tiab] OR fowl[Tiab] OR fowls[Tiab] OR reptile[Tiab] OR reptilia[Tiab] OR reptiles[Tiab] OR snakes[Tiab] OR snake[Tiab] OR lizard[Tiab] OR lizards[Tiab] OR alligator[Tiab] OR alligators[Tiab] OR crocodile[Tiab] OR crocodiles[Tiab] OR turtle[Tiab] OR turtles[Tiab] OR amphibian[Tiab] OR amphibians[Tiab] OR amphibia[Tiab] OR frog[Tiab] OR frogs[Tiab] OR bombina[Tiab] OR salientia[Tiab] OR toad[Tiab] OR toads[Tiab] OR "epidalea calamita"[Tiab] OR salamander[Tiab] OR salamanders[Tiab] OR eel[Tiab] OR eels[Tiab] OR fish[Tiab] OR fishes[Tiab] OR pisces[Tiab] OR catfish[Tiab] OR catfishes[Tiab] OR siluriformes[Tiab] OR arius[Tiab] OR heteropneustes[Tiab] OR sheatfish[Tiab] OR perch[Tiab] OR perches[Tiab] OR percidae[Tiab] OR perca[Tiab] OR trout[Tiab] OR trouts[Tiab] OR char[Tiab] OR chars[Tiab] OR salvelinus[Tiab] OR minnow[Tiab] OR cyprinidae[Tiab] OR carps[Tiab] OR carp[Tiab] OR zebrafish[Tiab] OR zebrafishes[Tiab] OR goldfish[Tiab] OR goldfishes[Tiab] OR guppy[Tiab] OR guppies[Tiab] OR chub[Tiab] OR chubs[Tiab] OR tinca[Tiab] OR barbels[Tiab] OR barbus[Tiab] OR pimephales[Tiab] OR promelas[Tiab] OR "poecilia reticulata"[Tiab] OR mullet[Tiab] OR mullets[Tiab] OR eel[Tiab] OR eels[Tiab] OR seahorse[Tiab] OR seahorses[Tiab] OR mugil curema[Tiab] OR atlantic cod[Tiab] OR shark[Tiab] OR sharks[Tiab] OR catshark[Tiab] OR anguilla[Tiab] OR salmonid[Tiab] OR salmonids[Tiab] OR whitefish[Tiab] OR whitefishes[Tiab] OR salmon[Tiab] OR salmons[Tiab] OR sole[Tiab] OR solea[Tiab] OR lamprey[Tiab] OR lampreys[Tiab] OR pumpkinseed[Tiab] OR sunfish[Tiab] OR sunfishes[Tiab] OR tilapia[Tiab] OR tilapias[Tiab] OR turbot[Tiab] OR turbots[Tiab] OR flatfish[Tiab] OR flatfishes[Tiab] OR sciuridae[Tiab] OR squirrel[Tiab] OR squirrels[Tiab] OR chipmunk[Tiab] OR chipmunks[Tiab] OR suslik[Tiab] OR susliks[Tiab] OR vole[Tiab] OR voles[Tiab] OR lemming[Tiab] OR lemmings[Tiab] OR muskrat[Tiab] OR muskrats[Tiab] OR lemmus[Tiab] OR otter[Tiab] OR otters[Tiab] OR marten[Tiab] OR martens[Tiab] OR martes[Tiab] OR weasel[Tiab] OR badger[Tiab] OR badgers[Tiab] OR ermine[Tiab] OR mink[Tiab] OR minks[Tiab] OR sable[Tiab] OR sables[Tiab] OR gulo[Tiab] OR gulos[Tiab] OR wolverine[Tiab] OR wolverines[Tiab] OR mustela[Tiab] OR llama[Tiab] OR llamas[Tiab] OR alpaca[Tiab] OR alpacas[Tiab] OR camelid[Tiab] OR camelids[Tiab] OR guanaco[Tiab] OR guanacos[Tiab] OR chiroptera[Tiab] OR chiropteras[Tiab] OR bat[Tiab] OR bats[Tiab] OR fox[Tiab] OR foxes[Tiab] OR iguana[Tiab] OR iguanas[Tiab] OR xenopus laevis[Tiab] OR parakeet[Tiab] OR parakeets[Tiab] OR parrot[Tiab] OR parrots[Tiab] OR donkey[Tiab] OR donkeys[Tiab] OR mule[Tiab] OR mules[Tiab] OR zebra[Tiab] OR zebras[Tiab] OR shrew[Tiab] OR shrews[Tiab] OR bison[Tiab] OR bisons[Tiab] OR buffalo[Tiab] OR buffaloes[Tiab] OR deer[Tiab] OR deers[Tiab] OR bear[Tiab] OR bears[Tiab] OR panda[Tiab] OR pandas[Tiab] OR "wild hog"[Tiab] OR "wild boar"[Tiab] OR fitchew[Tiab] OR fitch[Tiab] OR beaver[Tiab] OR beavers[Tiab] OR jerboa[Tiab] OR jerboas[Tiab] OR capybara[Tiab] OR capybaras[Tiab] OR canine [tiab] OR bovine [tiab] OR porcine [tiab] OR hog [tiab] OR hogs [tiab]) NOT medline[sb])))

b

Embase (OVID)

1 exp surgical mesh/ or mesh* OR prothes* or prosthet* or exp transabdominal mesh/

2 (coated or coating or covered or covering or composite or intraperitoneal or transabdominal or intraperitoneal or intra-abdominal or IPOM).ti,ab.

31 and 2

4 (Sepracoat or sepramesh or seprafilm or Parietex or hyaluronate carboxymethylcellulose or Proceed or Vypro or Gore-tex mesh or Dual mesh or Composix or Dynamesh or Ultrapro or Ti-mesh or CQur).ti,ab.

53 or 4

6 (Abdominal or Ventral or incisional or Incisive or Inguinal or Parastomal or Femoral or Umbilical or Epigastric or Spigelian or Postoperative or Laparotomy or Laparotomies or Celiotomy or Celiotomies or Minilaparotomy or Minilaparotomies).ti,ab.

7 (hernia or hernias or defect or defects or herniorrhaphy or hernioplasty).ti,ab.

$8 \quad 6$ and 7 
9 exp incisional hernia/ or exp spigelian hernia/ or exp abdominal wall hernia/ or exp inguinal hernia/ or exp parastomal hernia/ or exp femoral hernia/ or exp umbilical hernia/

108 or 9

11 exp animal experiment/ or exp animal model/ or exp experimental animal/ or exp transgenic animal/ or exp male animal/ or exp female animal/ or exp juvenile animal/ or animal/ or chordata/ or vertebrate/ or tetrapod/ or exp fish/ or amniote/ or exp amphibia/ or mammal/ or exp reptile/ or exp sauropsid/ or therian/ or exp monotremate/ or placental mammals/ or exp marsupial/ or Euarchontoglires/ or exp Afrotheria/ or exp Boreoeutheria/ or exp Laurasiatheria/ or exp Xenarthra/ or primate/ or exp Dermoptera/ or exp Glires/ or exp Scandentia/ or Haplorhini/ or exp prosimian/ or simian/ or exp tarsiiform/ or Catarrhini/ or exp Platyrrhini/ or ape/ or exp Cercopithecidae/ or hominid/ or exp hylobatidae/ or exp chimpanzee/ or exp gorilla/ or exp orang utan/ or (animal or animals or pisces or fish or fishes or catfish or catfishes or sheatfish or silurus or arius or heteropneustes or clarias or gariepinus or fathead minnow or fathead minnows or pimephales or promelas or cichlidae or trout or trouts or char or chars or salvelinus or salmo or oncorhynchus or guppy or guppies or millionfish or poecilia or goldfish or goldfishes or carassius or auratus or mullet or mullets or mugil or curema or shark or sharks or cod or cods or gadus or morhua or carp or carps or cyprinus or carpio or killifish or eel or eels or anguilla or zander or sander or lucioperca or stizostedion or turbot or turbots or psetta or flatfish or flatfishes or plaice or pleuronectes or platessa or tilapia or tilapias or oreochromis or sarotherodon or common sole or dover sole or solea or zebrafish or zebrafishes or danio or rerio or seabass or dicentrarchus or labrax or morone or lamprey or lampreys or petromyzon or pumpkinseed or pumpkinseeds or lepomis or gibbosus or herring or clupea or harengus or amphibia or amphibian or amphibians or anura or salientia or frog or frogs or rana or toad or toads or bufo or xenopus or laevis or bombina or epidalea or calamita or salamander or salamanders or newt or newts or triturus or reptilia or reptile or reptiles or bearded dragon or pogona or vitticeps or iguana or iguanas or lizard or lizards or anguis fragilis or turtle or turtles or snakes or snake or aves or bird or birds or quail or quails or coturnix or bobwhite or colinus or virginianus or poultry or poultries or fowl or fowls or chicken or chickens or gallus or zebra finch or taeniopygia or guttata or canary or canaries or serinus or canaria or parakeet or parakeets or grasskeet or parrot or parrots or psittacine or psittacines or shelduck or tadorna or goose or geese or branta or leucopsis or woodlark or lullula or flycatcher or ficedula or hypoleuca or dove or doves or geopelia or cuneata or duck or ducks or greylag or graylag or anser or harrier or circus pygargus or red knot or great knot or calidris or canutus or godwit or limosa or lapponica or meleagris or gallopavo or jackdaw or corvus or monedula or ruff or philomachus or pugnax or lapwing or peewit or plover or vanellus or swan or cygnus or columbianus or bewickii or gull or chroicocephalus or ridibundus or albifrons or great tit or parus or aythya or fuligula or streptopelia or risoria or spoonbill or platalea or leucorodia or blackbird or turdus or merula or blue tit or cyanistes or pigeon or pigeons or columba or pintail or anas or starling or sturnus or owl or athene noctua or pochard or ferina or cockatiel or nymphicus or hollandicus or skylark or alauda or tern or sterna or teal or crecca or oystercatcher or haematopus or ostralegus or shrew or shrews or sorex or araneus or crocidura or russula or european mole or talpa or chiroptera or bat or bats or eptesicus or serotinus or myotis or dasycneme or daubentonii or pipistrelle or pipistrellus or cat or cats or felis or catus or feline or dog or dogs or canis or canine or canines or otter or otters or lutra or badger or badgers or meles or fitchew or fitch or foumart or foulmart or ferrets or ferret or polecat or polecats or mustela or putorius or weasel or weasels or fox or foxes or vulpes or common seal or phoca or vitulina or grey seal or halichoerus or horse or horses or equus or equine or equidae or donkey or donkeys or mule or mules or pig or pigs or swine or swines or hog or hogs or boar or boars or porcine or piglet or piglets or sus or scrofa or llama or llamas or lama or glama or deer or deers or cervus or elaphus or cow or cows or bos taurus or bos indicus or bovine or bull or bulls or cattle or bison or bisons or sheep or sheeps or ovis aries or ovine or lamb or lambs or mouflon or mouflons or goat or goats or capra or caprine or chamois or rupicapra or leporidae or lagomorpha or lagomorph or rabbit or rabbits or oryctolagus or cuniculus or laprine or hares or lepus or rodentia or rodent or rodents or murinae or mouse or mice or mus or musculus or murine or woodmouse or apodemus or rat or rats or rattus or norvegicus or guinea pig or guinea pigs or cavia or porcellus or hamster or hamsters or mesocricetus or cricetulus or cricetus or gerbil or gerbils or jird or jirds or meriones or unguiculatus or jerboa or jerboas or jaculus or chinchilla or chinchillas or beaver or beavers 
or castor fiber or castor canadensis or sciuridae or squirrel or squirrels or sciurus or chipmunk or chipmunks or marmot or marmots or marmota or suslik or susliks or spermophilus or cynomys or cottonrat or cottonrats or sigmodon or vole or voles or microtus or myodes or glareolus or primate or primates or prosimian or prosimians or lemur or lemurs or lemuridae or loris or bush baby or bush babies or bushbaby or bushbabies or galago or galagos or anthropoidea or anthropoids or simian or simians or monkey or monkeys or marmoset or marmosets or callithrix or cebuella or tamarin or tamarins or saguinus or leontopithecus or squirrel monkey or squirrel monkeys or saimiri or night monkey or night monkeys or owl monkey or owl monkeys or douroucoulis or aotus or spider monkey or spider monkeys or ateles or baboon or baboons or papio or rhesus monkey or macaque or macaca or mulatta or cynomolgus or fascicularis or green monkey or green monkeys or chlorocebus or vervet or vervets or pygerythrus or hominoidea or ape or apes or hylobatidae or gibbon or gibbons or siamang or siamangs or nomascus or symphalangus or hominidae or orangutan or orangutans or pongo or chimpanzee or chimpanzees or pan troglodytes or bonobo or bonobos or pan paniscus or gorilla or gorillas or troglodytes).ti,ab.

125 and 10 and $11=$ articles searched in Embase 
Table S8.2 The comparison between polypropylene mesh and HA/CMC coated PP mesh (PP mesh VS PP mesh + HA/CMC).

\begin{tabular}{|c|c|c|c|c|}
\hline Species & Follow-up & $\begin{array}{l}\text { Comparison } \\
(\mathrm{PP} \text { mesh VS PP mesh + HA/CMC) }\end{array}$ & Best mesh & Measurements and reference \\
\hline Rat & $\begin{array}{l}\text { Several time } \\
\text { points } \\
\text { together till } 4 \\
\text { weeks }\end{array}$ & $\begin{array}{l}\text { Surgipromesh (AutoSuture) VS PP mesh }+ \\
\mathrm{CMC}^{1} \text {; } \\
\text { PP mesh VS Sepramesh (Genzyme) })^{2}\end{array}$ & $\mathrm{PP}$ mesh + HA/CMC & $\begin{array}{l}\text { Extent score of adhesion }{ }^{1} ; \\
\text { Rate of adhesion presence } \\
\text { Percentage of adhesion-area }\end{array}$ \\
\hline Rat & 1 week & $\begin{array}{l}\text { Prolene (Ethicon) VS PP mesh }+\mathrm{HA}^{3} ; \\
\text { Prolene (Ethicon) VS Sepramesh } \\
(\text { Genzyme) })^{4,5} \\
\text { Marlex (Bard) VS PP mesh }+ \text { Seprafilm } \\
(\text { Genzyme })^{6}\end{array}$ & $\mathrm{PP}$ mesh + HA/CMC & $\begin{array}{l}\text { Tenacity score of adhesion } \\
\text { Numbers of rats with bowel adhesion } \\
\text {; } \\
\text { Percentage of adhesion-area }\end{array}$ \\
\hline $\begin{array}{l}\text { Rat } \\
\text { Rabbits }\end{array}$ & 2 weeks & $\begin{array}{l}\text { PP mesh VS PP mesh + Seprafilm } \\
(\text { Genzyme })^{7} ; \\
\text { PP mesh VS PP mesh + Sepracoat } \\
(\text { Genzyme })^{8} \text {; } \\
\text { Marlex (Bard) VS Sepramesh }(\text { Genzyme })^{9} \text {; } \\
\text { Marlex (Bard) VS PP mesh + Seprafilm } \\
(\text { Genzyme })^{6}\end{array}$ & $\mathrm{PP}$ mesh + HA/CMC & $\begin{array}{l}\text { Percentage of adhesion-area }{ }^{7} \text { (rat), }{ }^{8,9} \text { (rabbit), }{ }^{6} \text { (rat); } \\
\text { Adhesion score composed of type and tenacity }{ }^{9} \\
\text { (rabbit) }\end{array}$ \\
\hline Rat & 3 weeks & $\begin{array}{l}\text { PP mesh VS PP mesh }+ \text { Seprafilm } \\
\text { (Genzyme) }\end{array}$ & $\mathrm{PP}$ mesh + HA/CMC & Percentage of adhesion-area ${ }^{10}$ \\
\hline Rat & 3 weeks & $\begin{array}{l}\text { Parietene (Sofradim) VS Sepramesh } \\
\text { (Genzyme) }\end{array}$ & No preference & $\begin{array}{l}\text { Adhesion-area } \\
\text { Rate of adhesion presence } \\
\text { Ri }\end{array}$ \\
\hline $\begin{array}{l}\text { Rat } \\
\text { Rabbit } \\
\text { Pig }\end{array}$ & 4 weeks & 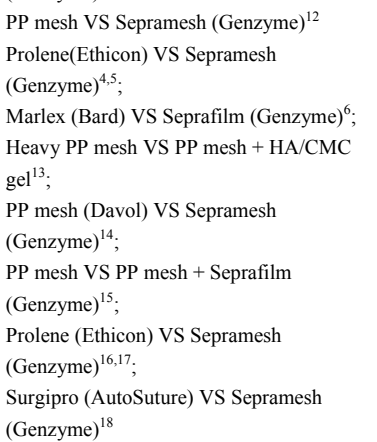 & $\mathrm{PP}$ mesh $+\mathrm{HA} / \mathrm{CMC}$ & $\begin{array}{l}\text { Percentage of adhesion-area }{ }^{12} \text { (pig), }{ }^{4-6,13} \text { (rat), },^{14,15} \\
\text { (rabbit); } \\
\text { Tenacity score of adhesion }{ }^{16-18} \text { (rat); } \\
\text { Extent score of adhesion }{ }^{13,17,18} \text { (rat), }{ }^{14} \text { (rabbit); } \\
\text { Severity score of adhesion }{ }^{14} \text { (rabbit), }{ }^{13} \text { (rat); } \\
\text { Density score of adhesion }{ }^{13} \text { (rat); } \\
\text { Rate of adhesion presence }{ }^{14} \text { (rabbit); } \\
\text { Adhesion numbers from organs to } \text { mesh }^{15} \text { (rabbit) }\end{array}$ \\
\hline $\begin{array}{l}\text { Rat } \\
\text { Pig }\end{array}$ & $\begin{array}{l}4 \text { weeks } \\
6 \text { weeks }\end{array}$ & $\begin{array}{l}\text { Prolene (Ethicon) VS PP mesh }+ \text { SCMC } \\
\text { Surgipro VS Seprafilm }(\text { Genzyme })^{20} \text {; } \\
\text { Premilene (Braun) VS PP mesh + Seprafilm } \\
(\text { Genzyme })^{21} \text {; } \\
\text { PP mesh VS PP mesh + Seprafilm } \\
(\text { Genzyme })^{10}\end{array}$ & $\begin{array}{l}\mathrm{PP} \text { mesh }+\mathrm{SCMC} \\
\mathrm{PP} \text { mesh }+\mathrm{HA} / \mathrm{CMC}\end{array}$ & $\begin{array}{l}\text { Extent score of adhesion }{ }^{19} \\
\text { Percentage of adhesion-area }{ }^{20} \text { (pig), }{ }^{10} \text { (rat); } \\
\text { Rate of adhesion presence }{ }^{21} \text { (rat) }\end{array}$ \\
\hline Rat & 8 weeks & $\begin{array}{l}\text { Prolene (Ethicon) VS Sepramesh } \\
(\text { Genzyme })^{22} \text {; } \\
\text { PP mesh VS Seprafilm (Genzyme) })^{23}\end{array}$ & $\mathrm{PP}$ mesh + HA/CMC & $\begin{array}{l}\text { Percentage of adhesion-area } \\
\text { Adhesion score composed of type and tenacity } \\
\text { Adhesion score composed of extent and tenacity } \\
\text { Number of rats with severity score of adhesion } \\
3-4^{22}\end{array}$ \\
\hline Rat & 3 months & Parietene (Covidien) VS Sepramesh (Bard) & $\mathrm{PP}$ mesh $+\mathrm{HA} / \mathrm{CMC}$ & $\begin{array}{l}\text { Percentage of adhesion-area } \\
\text { Adhesion score composed of extent, type and } \\
\text { tenacity }{ }^{24}\end{array}$ \\
\hline Rabbit & 4 months & ProLite Ultra mesh VS Sepramesh (Bard) & $\mathrm{PP}$ mesh + HA/CMC & $\begin{array}{l}\text { Percentage of adhesion-area } \\
\text { Tenacity score of adhesion } \\
\text { Th }^{25}\end{array}$ \\
\hline
\end{tabular}


Table S8.3 The comparison between PP mesh and Parietex Composite mesh (PP mesh VS Polyester mesh + collagen).

\begin{tabular}{|c|c|c|c|c|}
\hline Species & Follow-up & $\begin{array}{l}\text { Comparison } \\
(\mathrm{PP} \text { mesh VS Polyester mesh }+ \text { collagen })\end{array}$ & Best mesh & Measurements and reference \\
\hline Rat & 1 week & $\begin{array}{l}\text { Prolene (Ethicon) VS Parietex Composite } \\
\text { (Sofradim) }\end{array}$ & No preference & Percentage of adhesion-area $^{5}$ \\
\hline Rat & 1 week & $\begin{array}{l}\text { Prolene (Ethicon) VS Parietex Composite } \\
(\text { Sofradim) })^{4,5,26}\end{array}$ & $\begin{array}{l}\text { Polyester mesh }+ \\
\text { collagen }\end{array}$ & $\begin{array}{l}\text { Numbers of rats with bowel adhesion } \\
\text { Percentage of adhesion-area } \\
4,26 \\
\text { Adhesion score composed of extent, type and } \\
\text { tenacity }^{26}\end{array}$ \\
\hline Rabbit & 2 weeks & $\begin{array}{l}\text { Surgipro (Tyco) VS Parietex Composite } \\
(\text { Sofradim })^{27} ; \\
\text { Prolene (Ethicon) VS Parietex Composite } \\
(\text { Sofradim })^{28}\end{array}$ & $\begin{array}{l}\text { Polyester mesh }+ \\
\text { collagen }\end{array}$ & $\begin{array}{l}\text { Percentage of adhesion-area }{ }^{27} \\
\text { Adhesion-area }^{28}\end{array}$ \\
\hline Rat & 3 weeks & $\begin{array}{l}\text { Parietene (Sofradim) VS Parietex Composite } \\
\text { (Sofradim) }^{11} \text {; } \\
\text { Marlex VS Parietex Composite }{ }^{29}\end{array}$ & $\begin{array}{l}\text { Polyester mesh }+ \\
\text { collagen }\end{array}$ & $\begin{array}{l}\text { Percentage of adhesion-area } \\
\text { Rate of adhesion presence }^{11,29} \\
\text { Tensile strength of adhesion }\end{array}$ \\
\hline Rat & 4 weeks & $\begin{array}{l}\text { Marlex VS Parietex Composite } \\
\text { Prolene (Ethicon) VS Parietex Composite } \\
\text { (Covidien) }^{31}\end{array}$ & No preference & $\begin{array}{l}\text { Percentage of adhesion-area }{ }^{30,31} \text {; } \\
\text { Extent score of adhesion } \\
\text { Tenacity score of adhesion }\end{array}$ \\
\hline Rat & 4 weeks & $\begin{array}{l}\text { Prolene (Ethicon) VS Parietex Composite } \\
\text { (Sofradim) })^{4,26} ; \\
\text { Prolene (Ethicon) VS Parietex Composite } \\
\text { (Covidien) }^{32} ; \\
\text { Surgipro (AutoSuture) VS Parietex } \\
\text { Composite (Sofradim) })^{18}\end{array}$ & $\begin{array}{l}\text { Polyester mesh }+ \\
\text { collagen }\end{array}$ & $\begin{array}{l}\text { Percentage of adhesion-area }{ }^{4,26,32} ; \\
\text { Extent score of adhesion }^{18} ; \\
\text { Tenacity score of adhesion } \\
\text { Adhesion score composed of extent, type and } \\
\text { tenacity }^{26} \text {; } \\
\text { Adhesion score composed of tenacity and severity }{ }^{32}\end{array}$ \\
\hline Rat & 3 months & $\begin{array}{l}\text { Prolene (Ethicon) VS Parietex Composite } \\
(\text { Covidien) })^{32} \text {; } \\
\text { Parietene (Covidien) VS Parietex Composite } \\
(\text { Covidien })^{24}\end{array}$ & $\begin{array}{l}\text { Polyester mesh }+ \\
\text { collagen }\end{array}$ & $\begin{array}{l}\text { Adhesion score composed of tenacity and } \\
\text { severity }^{32} \text {; } \\
\text { Percentage of adhesion-area }{ }^{32} \text {; } \\
\text { Adhesion score composed of extent, type and } \\
\text { tenacity }^{24}\end{array}$ \\
\hline
\end{tabular}

Table S8.4 The comparison between PP mesh and Parietene Composite mesh (PP mesh VS PP mesh + collagen).

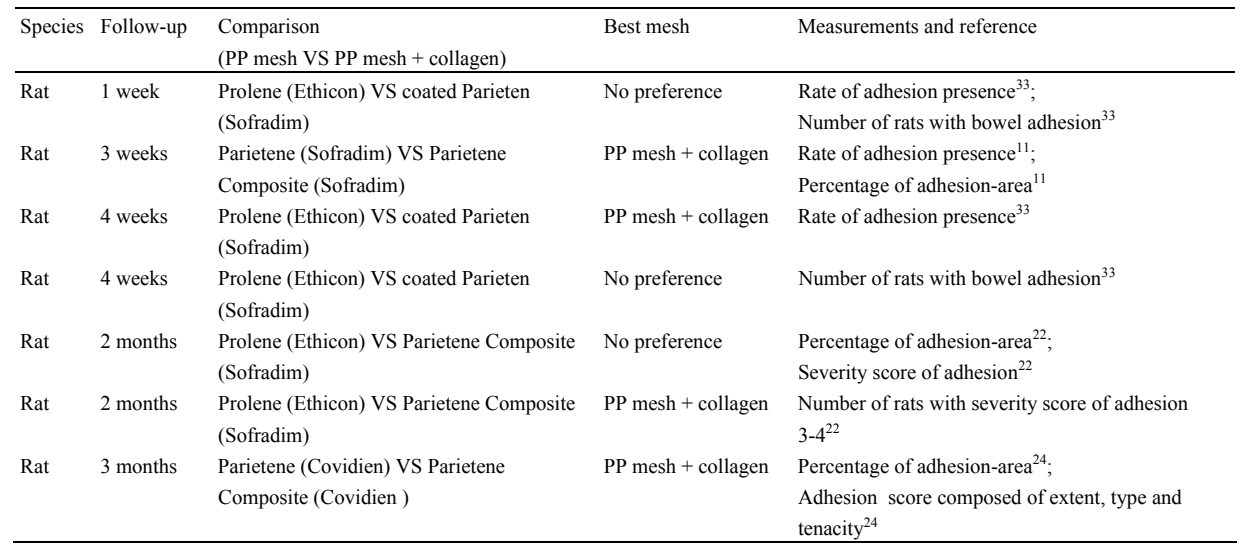


Table S8.5 The comparison between PP mesh and PP mesh coated by polyurethane (PP mesh VS PP mesh + PU).

\begin{tabular}{|c|c|c|c|c|}
\hline Species & Follow-up & $\begin{array}{l}\text { Comparison } \\
(\mathrm{PP} \text { mesh VS PP mesh + PU) }\end{array}$ & Best mesh & Measurements and reference \\
\hline Rat & 2 weeks & Marlex (Bard) VS PP mesh + PU & No preference & Percentage of adhesion-area ${ }^{34}$ \\
\hline $\begin{array}{l}\text { Rat } \\
\text { Rabbit }\end{array}$ & 2 weeks & $\begin{array}{l}\text { Marlex (Bard) VS PP mesh + } \mathrm{PU}^{34} \\
\text { Surgipro (Tyco) VS PP mesh }+\mathrm{PU}^{27}\end{array}$ & $\mathrm{PP}$ mesh $+\mathrm{PU}$ & $\begin{array}{l}\text { Severity score of adhesion }(\geq \text { moderate })^{34} \text {; } \\
\text { Percentage of adhesion-area }{ }^{27}\end{array}$ \\
\hline Rat & $\begin{array}{l}3 \text { weeks, } \\
3 \text { months, } \\
6 \text { months } \\
\text { together }\end{array}$ & Prolene (Ethicon) VS Combi (Angiologica) & $\mathrm{PP}$ mesh $+\mathrm{PU}$ & $\begin{array}{l}\text { Extent score of adhesion }{ }^{36} \\
\text { Tenacity score of adhesion }\end{array}$ \\
\hline
\end{tabular}

Table S8.6 The comparison between PP mesh and PP-polydioxanone mesh coated by oxidized regenerated cellulose (PP mesh VS PP mesh + ORC).

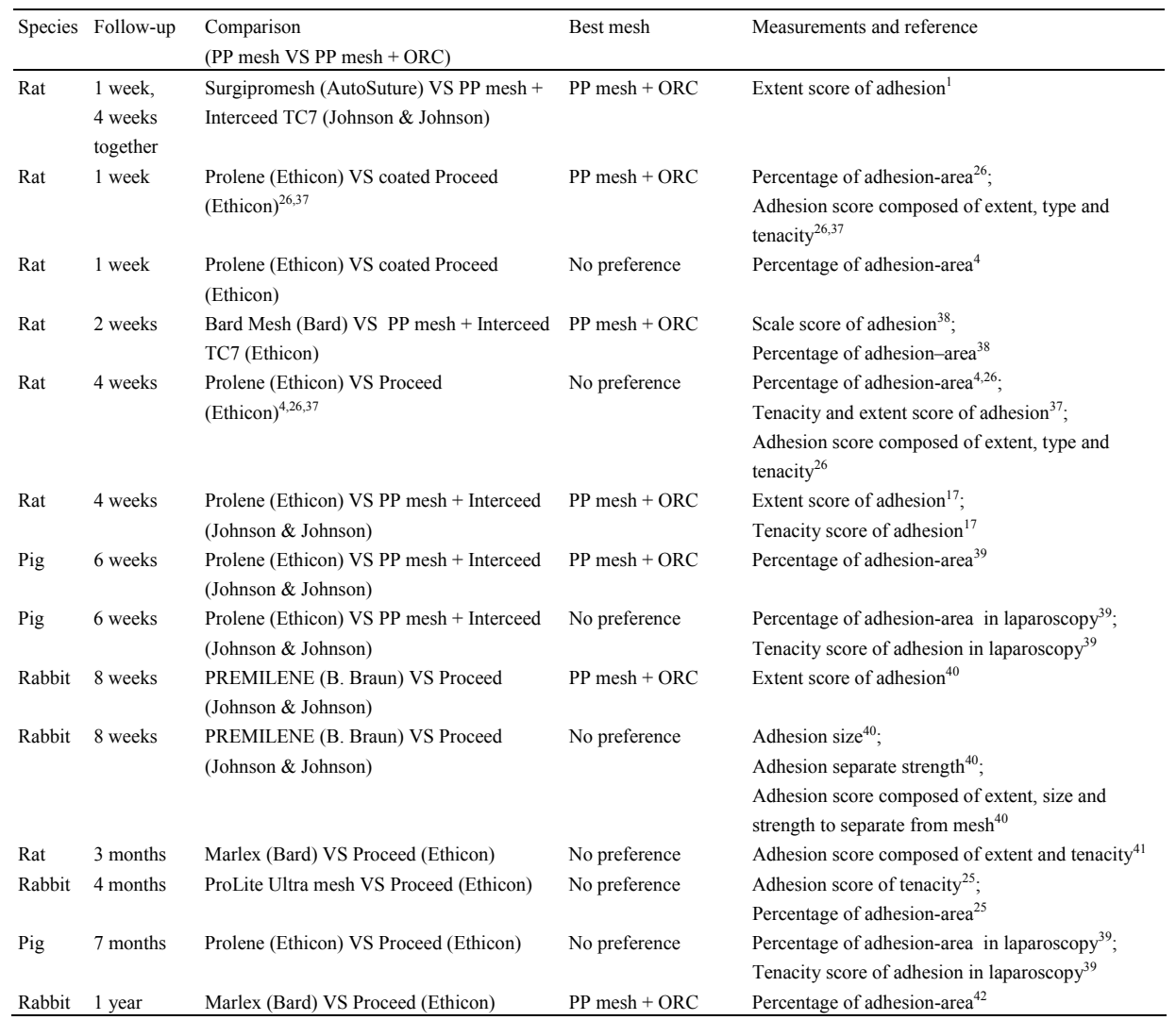


Table S8.7 The comparison between PP mesh and polyglactin coated PP mesh (PP mesh VS PP mesh + polyglactin).

\begin{tabular}{|c|c|c|c|c|}
\hline Species & Follow-up & $\begin{array}{l}\text { Comparison } \\
\text { (PP mesh VS PP mesh }+ \text { polyglactin })\end{array}$ & Best mesh & Measurements and reference \\
\hline Rabbit & 2 weeks & $\begin{array}{l}\text { Marlex(Bard) VS Marlex (Bard) + Vicryl } \\
\text { (Ethicon) }\end{array}$ & PP mesh + polyglactin $\mathrm{I}$ & $\begin{array}{l}\text { Percentage of adhesion-area"; } \\
\text { Adhesion score composed of type and tenacity }\end{array}$ \\
\hline Rat & 2 weeks & $\begin{array}{l}\text { Parietene (Tyco) VS VyproII (Ethicon); } \\
\text { Optilene (B/Braun) VS VyproII (Ethicon) }\end{array}$ & No preference & Percentage of adhesion-area ${ }^{43}$ \\
\hline Rat & 4 weeks & $\begin{array}{l}\text { Surgipro mesh (AutoSuture) VS Vypro II } \\
\text { (Johnson \& Johnson) }\end{array}$ & PP mesh + polyglactin & $\begin{array}{l}\text { Extent score of adhesion }\left({ }^{18}\right) \text {; } \\
\text { Tenacity score of adhesion }{ }^{(18)} \text {; } \\
\text { Breaking force of adhesion }{ }^{(18)}\end{array}$ \\
\hline Rabbit & 3 months & PP mesh VS PP mesh + polyglactin & \multicolumn{2}{|c|}{ PP mesh + polyglactin Percentage of adhesion-area $\left({ }^{43}\right)$} \\
\hline
\end{tabular}

Table S8.8 The comparison between PP mesh and PP mesh covered by expanded polytetrafluoroethylene (PP mesh VS PP mesh + ePTFE).

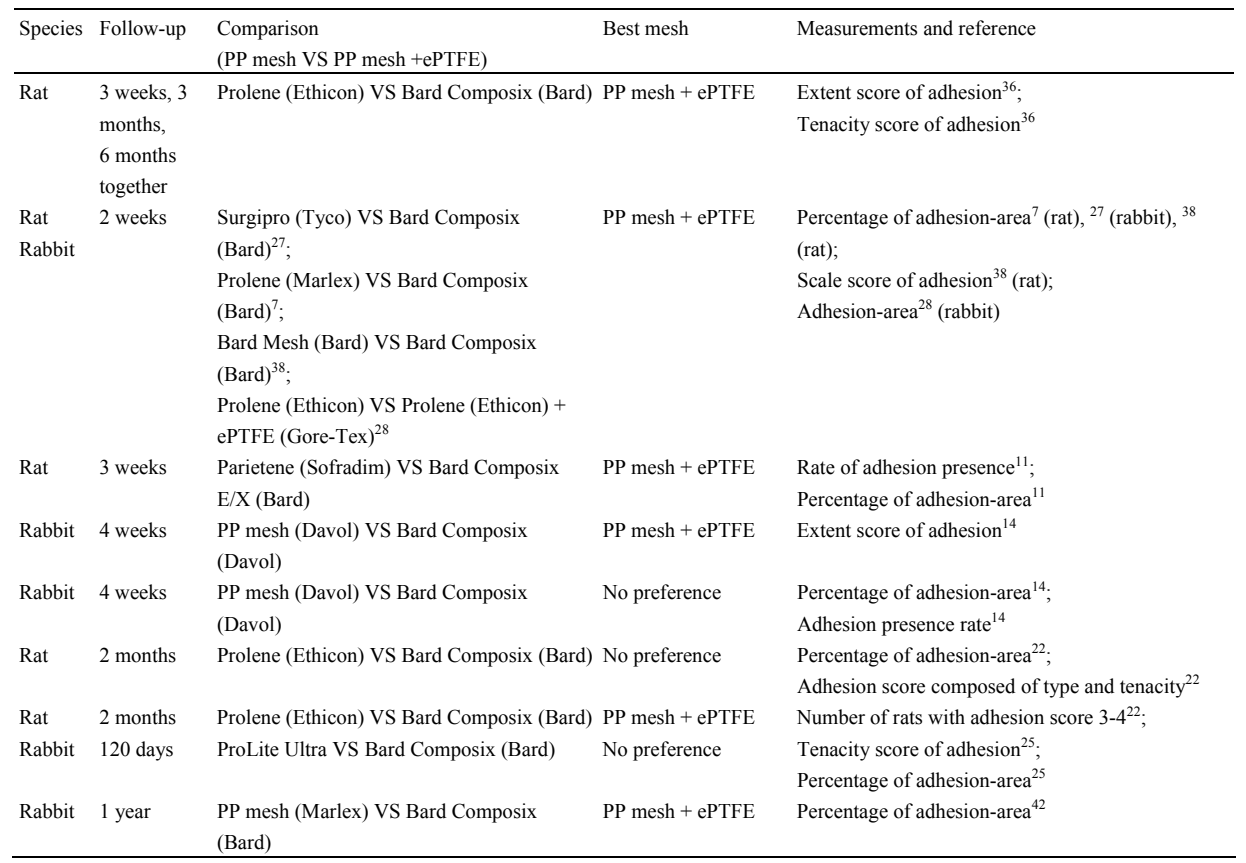


Table S8.9 The comparison between PP mesh and C-Qur mesh (PP mesh VS C-QUR mesh).

\begin{tabular}{|c|c|c|c|c|}
\hline Species & Follow-up & $\begin{array}{l}\text { Comparison } \\
\text { (PP mesh VS C-QUR mesh) }\end{array}$ & Best mesh & Measurements and reference \\
\hline Rat & 7 days & Prolene (Ethicon) VS C-Qur (Atrium) & C-Qur mesh & $\begin{array}{l}\text { Percentage of adhesion-area } \\
\text { Adhesion score composed of extent, type and } \\
\text { tenacity }^{26}\end{array}$ \\
\hline Rat & 30 days & Prolene (Ethicon) VS C-Qur (Atrium) & C-Qur mesh & $\begin{array}{l}\text { Percentage of adhesion-area } \\
{ }^{26} \\
\text { Adhesion score composed of extent, type and } \\
\text { tenacity }^{26}\end{array}$ \\
\hline Rat & 90 days & Parietene (Covidien) VS C-Qur (Atrium) & C-Qur mesh & $\begin{array}{l}\text { Percentage of adhesion-area }{ }^{24} \text {; } \\
\text { Adhesion score composed of extent, type and } \\
\text { tenacity }^{24}\end{array}$ \\
\hline Rabbit & 120 days & ProLite Ultra VS C-Qur (Atrium) & No preference & $\begin{array}{l}\text { Tenacity score of adhesion } \\
{ }^{25} \\
\text { Percentage of adhesion-area }^{25}\end{array}$ \\
\hline
\end{tabular}

Table S8.10 The comparison between PP mesh coated by collagen (Parietene Composite mesh) VS other meshes with coatings.

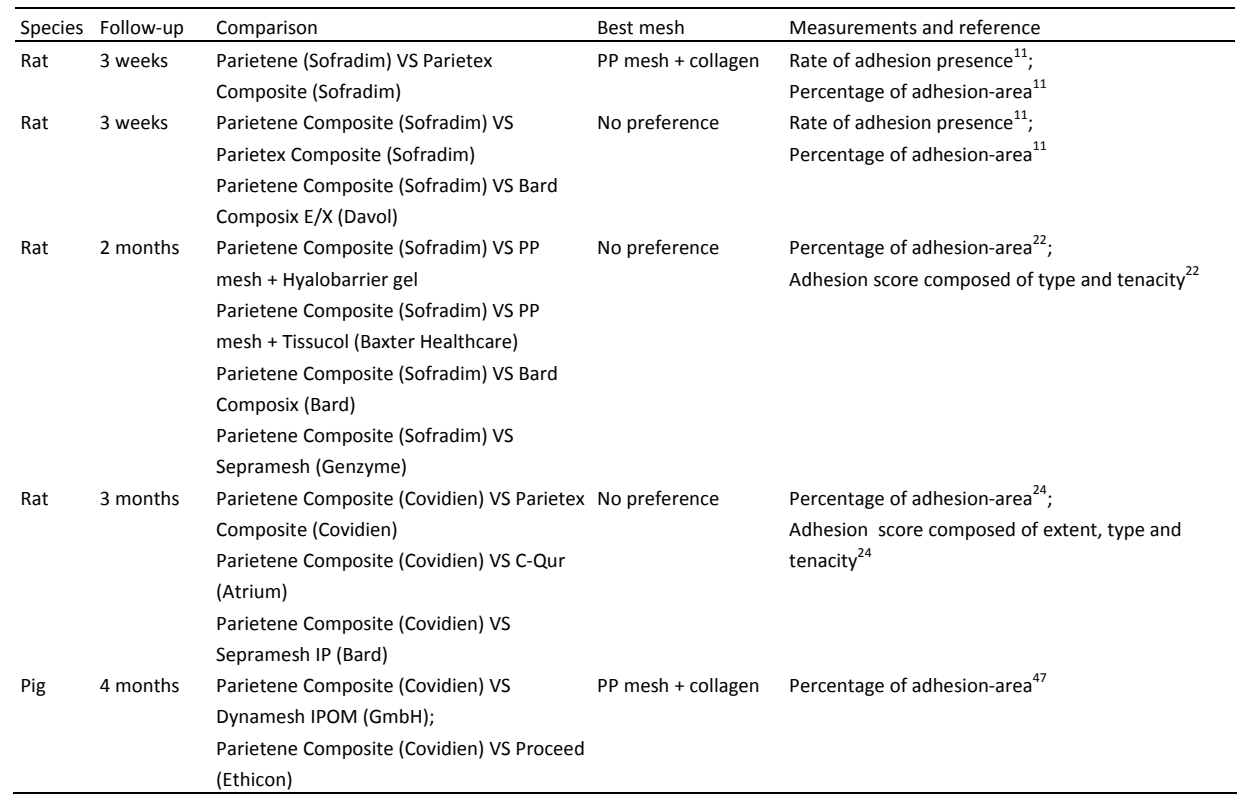


Table S8.11 The comparison between PP mesh coated by HA/CMC (Sepramesh) and other kinds of meshes with coatings

\begin{tabular}{|c|c|c|c|c|}
\hline Species & Follow-up & Comparison & Best mesh & Measurements and reference \\
\hline Rat & $\begin{array}{l}1 \text { week, } 4 \\
\text { weeks } \\
\text { together }\end{array}$ & $\begin{array}{l}\text { PP mesh + Interceed TC7 (Johnson \& } \\
\text { Johnson) VS PP mesh + CMC }\end{array}$ & No preference & Extent score of adhesion $^{1}$ \\
\hline Rabbit & 3 days & $\begin{array}{l}\text { Sepramesh (Genzyme) VS Parietex } \\
\text { Composite (Sofradim) } \\
\text { Sepramesh (Genzyme) VS PP mesh + } \\
\text { polyurethane }\end{array}$ & $\mathrm{PP}$ mesh + HA/CMC & Percentage of mesh adhesion-area ${ }^{48}$ \\
\hline Rabbit & 3 days & $\begin{array}{l}\text { Sepramesh (Bard) VS Parietex Composite } \\
\text { (Covidien) }\end{array}$ & $\begin{array}{l}\text { Polyester mesh + } \\
\text { collagen }\end{array}$ & Rate of adhesion presence ${ }^{49}$ \\
\hline Rabbit & 3 days & Sepramesh (Bard) VS Proceed (Ethicon) & No preference & Rate of adhesion presence ${ }^{49}$ \\
\hline $\begin{array}{l}\text { Rat } \\
\text { Rabbit }\end{array}$ & 1 week & $\begin{array}{l}\text { Sepramesh (Bard) VS Parietex Composite } \\
\text { (Covidien) }^{50} ; \\
\text { Sepramesh (Genzyme) VS Parietex } \\
\text { Composite (Sofradim) }{ }^{5} ; \\
\text { Sepramesh (Bard) VS Physiomesh } \\
\text { (Ethicon) }^{50} ; \\
\text { Sepramesh (Genzyme) VS DualMesh } \\
\text { (GORE-TEX) }^{51} \text {; } \\
\text { Sepramesh (Bard) VS Proceed (Ethicon) }\end{array}$ & No preference & $\begin{array}{l}\text { Percentage of adhesion-area }{ }^{5} \text { (rat); } \\
\text { Numbers of rats with bowel adhesion }{ }^{5} \text {; } \\
\text { Adhesion score composed of width, thickness, } \\
\text { subjective strength and amount of adhesion } \\
\text { (rabbit); } \\
\text { Extent score of adhesion }{ }^{51} \text { (rabbit); } \\
\text { Rate of adhesion presence }{ }^{49} \text { (rabbit) }\end{array}$ \\
\hline $\begin{array}{l}\text { Rat } \\
\text { Rabbit }\end{array}$ & 1 week & $\begin{array}{l}\text { Sepramesh (Genzyme) VS Ultrapro } \\
\text { (Ethicon) }^{4} \text {; } \\
\text { Sepramesh (Genzyme) VS Timesh (GfE } \\
\text { Medizintechnik GmbH) } \\
\text { Sepramesh (Bard) VS Bard Composix } \\
\text { (Bard) }^{50} \text {; } \\
\text { Sepramesh (Bard) VS Proceed (Johnson \& } \\
\text { Johnson) }{ }^{50} \text {; } \\
\text { Sepramesh (Genzyme) VS Parietex } \\
\text { Composite (Sofradim) }{ }^{48} \text {; } \\
\text { Sepramesh (Genzyme) VS PP mesh + } \\
\text { polyurethane }\end{array}$ & $\mathrm{PP}$ mesh + HA/CMC & $\begin{array}{l}\text { Percentage of adhesion-area }{ }^{48} \text { rabbit); } \\
\text { Adhesion score composed of width, thickness, } \\
\text { subjective strength and amount of } \\
\text { adhesion }{ }^{50} \text { (rabbit) }\end{array}$ \\
\hline Rabbit & 1 week & $\begin{array}{l}\text { Sepramesh (Bard) VS Parietex composite } \\
\text { (Covidien) }\end{array}$ & $\begin{array}{l}\text { Polyester mesh + } \\
\text { collagen }\end{array}$ & Rate of adhesion presence ${ }^{49}$ \\
\hline $\begin{array}{l}\text { Rat } \\
\text { Rabbit }\end{array}$ & 2 weeks & $\begin{array}{l}\text { PP mesh + Seprafilm (Genzyme) VS } \\
\text { Composix (Bard) }{ }^{7} \text {; } \\
\text { Sepramesh (Bard) VS Proceed (Ethicon })^{49} ; \\
\text { Sepramesh (Genzyme) VS Marlex (Bard) + } \\
\text { Vicryl }\left({ }^{9}{ }^{\text {) }}\right.\end{array}$ & No preference & $\begin{array}{l}\text { Percentage of adhesion-area } \\
\text { Percentage of adhesion-area } \\
\text { Adhesion score composed of type and tenacity }^{9} \text {; } \\
\text { Rate of adhesion presence }^{49}\end{array}$ \\
\hline Rabbit & 2 weeks & $\begin{array}{l}\text { Sepramesh (Genzyme) VS Parietex } \\
\text { Composite (Sofradim); } \\
\text { Sepramesh (Genzyme) VS PP mesh + } \\
\text { polyurethane }\end{array}$ & $\mathrm{PP}$ mesh + HA/CMC & Percentage of mesh adhesion-area ${ }^{48}$ \\
\hline Rabbit & 2 weeks & $\begin{array}{l}\text { Sepramesh (Bard) VS Parietex Composite } \\
\text { (Covidien) }\end{array}$ & $\begin{array}{l}\text { Polyester mesh + } \\
\text { collagen }\end{array}$ & Rate of adhesion presence ${ }^{49}$ \\
\hline Rat & 3 weeks & $\begin{array}{l}\text { Sepramesh (Genzyme) VS Parietex } \\
\text { Composite (Sofradim) }\end{array}$ & $\begin{array}{l}\text { Polyester mesh + } \\
\text { collagen }\end{array}$ & $\begin{array}{l}\text { Rate of adhesion presence }{ }^{11} \\
\text { Percentage of adhesion-area }\end{array}$ \\
\hline Rat & 3 weeks & $\begin{array}{l}\text { Sepramesh (Genzyme) VS Parietex } \\
\text { Composite (Sofradim) }\end{array}$ & PP mesh + collagen & $\begin{array}{l}\text { Rate of adhesion presence }{ }^{11} \\
\text { Percentage of adhesion-area }\end{array}$ \\
\hline $\begin{array}{l}\text { Rat } \\
\text { Rabbit }\end{array}$ & 3 weeks & $\begin{array}{l}\text { Sepramesh (Genzyme) VS Bard Composix } \\
\text { E/X (Davol) }{ }^{11} \text {; } \\
\text { Sepramesh (Genzyme) VS Bard Composix } \\
\text { (Bard) })^{51}\end{array}$ & No preference & $\begin{array}{l}\text { Rate of adhesion presence }{ }^{11} \\
\text { Extent score of adhesion }^{51}\end{array}$ \\
\hline
\end{tabular}


Table S8.11

(continued)

\begin{tabular}{|c|c|c|c|c|}
\hline Species & Follow-up & Comparison & Best mesh & Measurements and reference \\
\hline $\begin{array}{l}\text { Rat } \\
\text { Rabbit }\end{array}$ & 4 weeks & $\begin{array}{l}\text { Sepramesh (Genzyme) VS Parietex } \\
\text { composite (Sofradim) } \\
\text { Sepramesh (Genzyme) VS Vypro II (Johnson } \\
\text { \& Johnson) })^{18} ; \\
\text { Sepramesh (Genzyme) VS Bard Composix } \\
\text { mesh (Davol) }{ }^{14}\end{array}$ & $\mathrm{PP}$ mesh + HA/CMC & $\begin{array}{l}\text { Percentage of adhesion-area }{ }^{14} \text { (rabbit) },^{5} \text { (rat); } \\
\text { Breaking strength of graft-organ adhesions }{ }^{18} \text { (rat); } \\
\text { Adhesion presence rate }{ }^{14} \text { (rabbit) }\end{array}$ \\
\hline Rabbit & 4 weeks & $\begin{array}{l}\text { Sepramesh (Genzyme) VS Parietex } \\
\text { Composite (Sofradim) })^{52}\end{array}$ & $\begin{array}{l}\text { Polyester mesh + } \\
\text { collagen }\end{array}$ & $\begin{array}{l}\text { Rate of bowel adhesion presence }{ }^{52} \\
\text { Adhesion-area }^{52}\end{array}$ \\
\hline $\begin{array}{l}\text { Rat } \\
\text { Rabbit } \\
\text { Pig }\end{array}$ & 4 weeks & 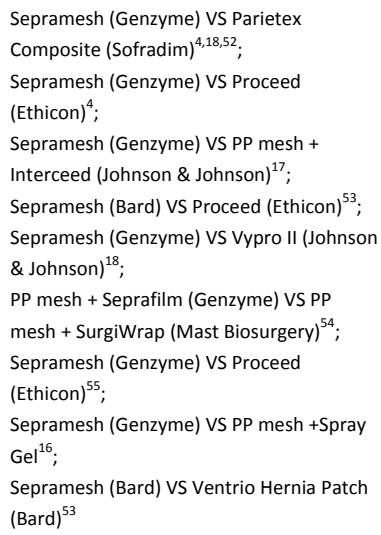 & No preference & $\begin{array}{l}\text { Percentage of adhesion-area }{ }^{4} \text { (rat), }{ }^{53} \text { (pig), } \\
\text { (rabbit); } \\
\text { Extent score of adhesion }{ }^{17} \text { (rat); } \\
\text { Tenacity score of adhesion }{ }^{53} \text { (pig), }\left(^{6,17} \text { (rat); }\right. \\
\text { Breaking strength of graft-omentum adhesions }{ }^{18} \\
\text { (rat); } \\
\text { Breaking strength of graft-organ adhesions }{ }^{18} \text { (rat); } \\
\text { Adhesion-area }{ }^{54} \text { (rat); } \\
\text { Adhesion score composed of density and scale }{ }^{55} \\
\text { (rat) }\end{array}$ \\
\hline Rabbit & 9 weeks & $\begin{array}{l}\text { Sepramesh (Genzyme) VS Bard Composix } \\
\text { (Bard) }\end{array}$ & No Preference & Extent score of adhesion ${ }^{51}$ \\
\hline Rat & 3 months & $\begin{array}{l}\text { Sepramesh (Genzyme) VS TiMesh (GfE } \\
\text { Medizintechnik GmbH) } \\
\text { Sepramesh (Genzyme) VS Vypro } \\
\text { (Ethicon) }^{56} \text {; } \\
\text { Sepramesh (Genzyme) VS DynaMesh IPOM } \\
\text { (FEG Textiltechnik) } \\
\text { Sepramesh (Bard) VS Parietex Composite } \\
\text { (Covidien) (M2) }{ }^{24} \text {; } \\
\text { Sepramesh (Bard) VS Parietene Composite } \\
\text { (Covidien) (M2,M3) } \\
\text { Sepramesh (Bard) VS C-Qur Edge (Atrium) } \\
\text { (M2) } \\
\text { Sepramesh (Bard) VS Intramesh T1 (Cousin) } \\
(\mathrm{M} 2)^{24}\end{array}$ & No preference & $\begin{array}{l}\text { Measurement1 (M1): Scale score of adhesion }{ }^{56} \text {; } \\
\text { Measurement2 (M2): Percentage of adhesion- } \\
\text { area }^{24} \text {; } \\
\text { Measurement3 (M3): Adhesion score composed of } \\
\text { extent, type and tenacity }{ }^{24}\end{array}$ \\
\hline Rat & 3 months & $\begin{array}{l}\text { Sepramesh (Bard) VS Parietex Composite } \\
\text { (Covidien) (M3); } \\
\text { Sepramesh (Bard) VS C-Qur Edge (M3); } \\
\text { Sepramesh (Bard) VS Intramesh T1 (M3); }\end{array}$ & $\mathrm{PP}$ mesh + HA/CMC & $\begin{array}{l}\text { Measurement2 (M2): Percentage of adhesion- } \\
\text { area }^{24} \text {; } \\
\text { Measurement3 (M3): Adhesion score composed of } \\
\text { extent, type and tenacity }{ }^{24}\end{array}$ \\
\hline Rabbit & 4 months & Sepramesh (Bard) VS Proceed (Ethicon) & $\mathrm{PP}$ mesh + HA/CMC & $\begin{array}{l}\text { Tenacity score of adhesion } \\
\text { Percentage of }^{25} \\
\text { Pdhesion-area }^{25}\end{array}$ \\
\hline Rabbit & 4 months & $\begin{array}{l}\text { Sepramesh (Bard) VS Parietex Composite } \\
\text { (Covidien) }^{25} \text {; } \\
\text { Sepramesh (Bard) VS Composix (Bard) }{ }^{25} ; \\
\text { Sepramesh (Genzyme) VS Composix (Bard) }{ }^{51} ; \\
\text { Sepramesh (Bard) VS C-Qur mesh (Atrium) }\end{array}$ & No preference & $\begin{array}{l}\text { Tenacity score of adhesion } \\
\text { Percentage of adhesion-area }^{25} \\
\text { Adhesion score of extent }^{51}\end{array}$ \\
\hline Rabbit & 5 months & $\begin{array}{l}\text { Sepramesh (Genzyme) VS Parietex } \\
\text { Composite (Sofradim) }\end{array}$ & No preference & Percentage of adhesion-area ${ }^{52}$ \\
\hline Rabbit & 5 months & $\begin{array}{l}\text { Sepramesh (Genzyme) VS Parietex } \\
\text { Composite (Sofradim) }\end{array}$ & $\begin{array}{l}\text { Polyester mesh + } \\
\text { collagen }\end{array}$ & $\begin{array}{l}\text { Rate of bowel adhesion presence } \\
\text { Adhesion-area }\end{array}$ \\
\hline
\end{tabular}


Table S8.12 The comparison between polyester mesh + collagen (Parietex composite mesh) and other meshes with coatings.

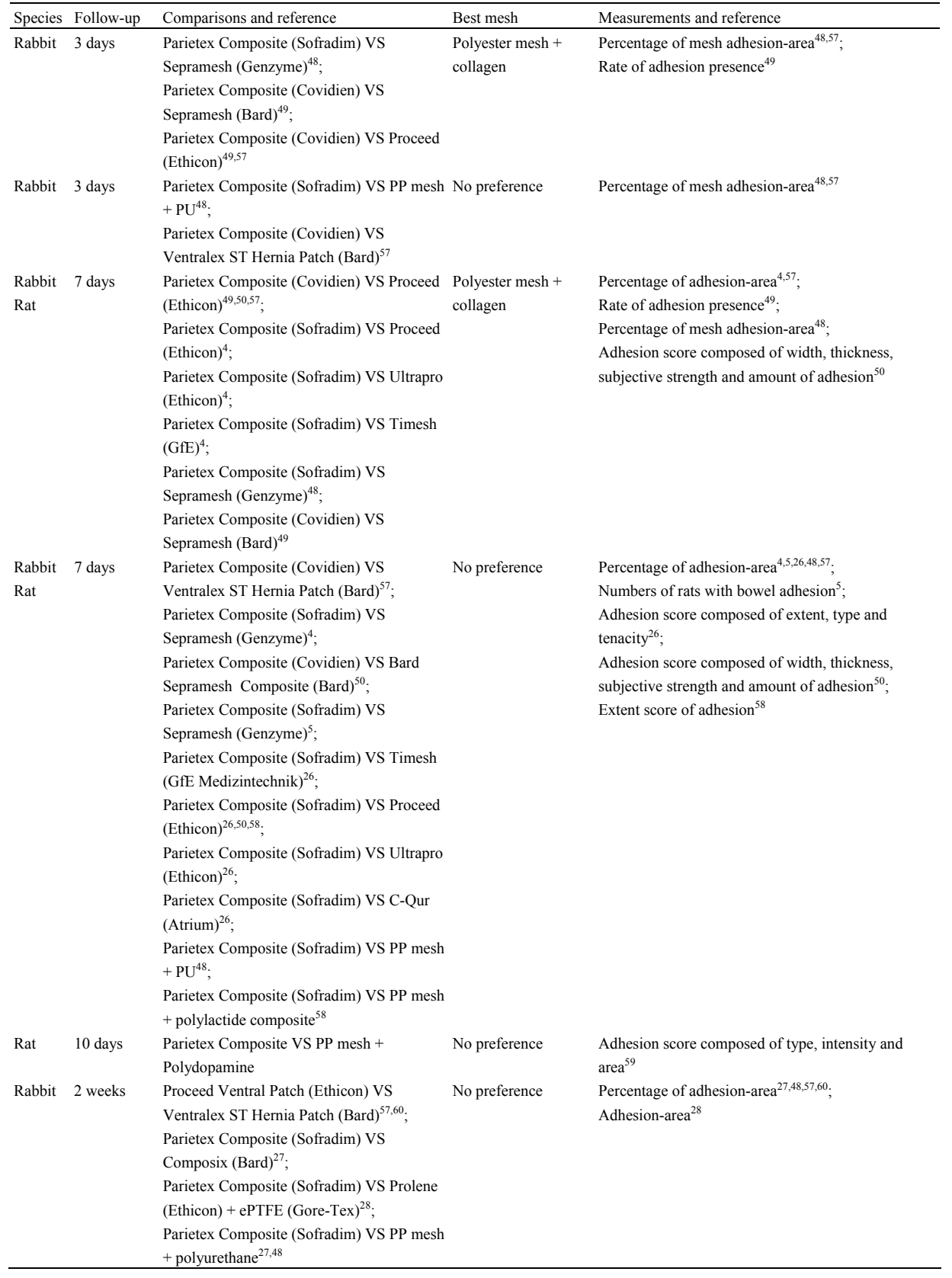


Table $\mathbf{S 8 . 1 2}$

(continued)

\begin{tabular}{|c|c|c|c|c|}
\hline Species & Follow-up & Comparisons and reference & Best mesh & Measurements and reference \\
\hline $\begin{array}{l}\text { Rabbit } \\
\text { Pig }\end{array}$ & 2 weeks & $\begin{array}{l}\text { Parietex Composite (Sofradim) VS } \\
\text { Sepramesh (Genzyme }{ }^{48} \text {; } \\
\text { Parietex Composite (Covidien) VS } \\
\text { Sepramesh (Bard) }{ }^{49} \text {; } \\
\text { Parietex Composite(Sofradim) VS Vypro } \\
(\text { Ethicon })^{61} \text {; } \\
\text { Parietex Composite Ventral Patch } \\
(\text { Covidien) VS Proceed Ventral Patch } \\
\text { (Ethicon) })^{57,60} \text {; } \\
\text { Parietex Composite (Sofradim) VS } \\
\text { Composix E/X (Bard) }{ }^{62}\end{array}$ & $\begin{array}{l}\text { Polyester mesh } \\
+ \text { collagen }\end{array}$ & $\begin{array}{l}\text { Percentage of mesh adhesion-area }{ }^{48,62} \text {; } \\
\text { Rate of adhesion presence }{ }^{49} \\
\text { Adhesion-area } \\
\text { Percentage of adhesion-area } \\
\text { Density score of adhesion }\end{array}$ \\
\hline Rat & 3 weeks & $\begin{array}{l}\text { Parietex Composite (Sofradim) VS } \\
\text { Sepramesh (Genzyme) }\end{array}$ & $\begin{array}{l}\text { Polyester mesh }+ \\
\text { collagen }\end{array}$ & $\begin{array}{l}\text { Rate of adhesion presence }{ }^{11} \\
\text { Percentage of adhesion-area }\end{array}$ \\
\hline Rat & 3 weeks & $\begin{array}{l}\text { Parietex Composite (Sofradim) VS } \\
\text { Parietene Composite (Sofradim); } \\
\text { Parietex Composite (Sofradim) VS Dual } \\
\text { Mesh (Gore-Tex) }\end{array}$ & No preference & $\begin{array}{l}\text { Rate of adhesion presence }{ }^{11} \\
\text { Percentage of adhesion-area }\end{array}$ \\
\hline Rat & 4 weeks & $\begin{array}{l}\text { Parietex composite (Sofradim) VS } \\
\text { Sepramesh (Genzyme) }\end{array}$ & $\mathrm{PP}$ mesh + HA/CMC & Percentage of adhesion-area ${ }^{5}$ \\
\hline Pig & 4 weeks & $\begin{array}{l}\text { Parietex Composite (Sofradim) VS Proceed } \\
\text { (Ethicon) }\end{array}$ & $\mathrm{PP}$ mesh + ORC & Percentage of adhesion-area ${ }^{63}$ \\
\hline $\begin{array}{l}\text { Rat } \\
\text { Rabbit }\end{array}$ & 4 weeks & $\begin{array}{l}\text { Parietex Composite (Covidien) VS Ultrapro } \\
\text { (Ethicon) } \\
\text { Parietex Composite (Covidien) VS Timesh } \\
(\text { GfE })^{4} ; \\
\text { Parietex Composite (Covidien) VS Proceed } \\
\text { (Ethicon) })^{4,26} \text {; } \\
\text { Parietex composite (Sofradim) VS Vypro II } \\
\text { (Johnson \& Johnson) }{ }^{18} ; \\
\text { Parietex composite (Sofradim) VS } \\
\text { Sepramesh (Genzyme })^{52} ; \\
\text { Parietex Composite }(\text { Covidie) VS PP mesh } \\
+ \text { polylactide composite }\end{array}$ & $\begin{array}{l}\text { Polyester mesh }+ \\
\text { collagen }\end{array}$ & $\begin{array}{l}\text { Percentage of adhesion-area }{ }^{4} \text { (rat); } \\
\text { Breaking strength of graft-organ }{ }^{18} \text { (rat); } \\
\text { Rate of bowel adhesion presence }{ }^{52} \text { (rabbit); } \\
\text { Adhesion-area }{ }^{52} \text { (rabbit); } \\
\text { Adhesion score composed of extent, type and } \\
\text { tenacity }^{26} \text { (rat) }\end{array}$ \\
\hline $\begin{array}{l}\text { Rat } \\
\text { Rabbit } \\
\text { Pig }\end{array}$ & 4 weeks & 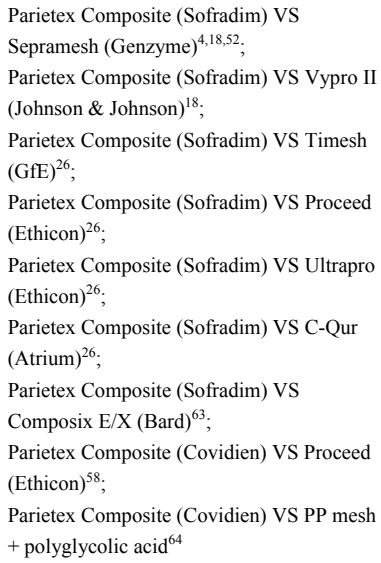 & No preference & $\begin{array}{l}\text { Percentage of adhesion-area }{ }^{4} \text { (rat), }{ }^{63} \text { (pig), }{ }^{52} \\
\text { (rabbit), }{ }^{26} \text { (rat); } \\
\text { Breaking strength of graft-omentum }{ }^{18} \text { (rat); } \\
\text { Extent score of adhesion }{ }^{58} \text { (rabbit); } \\
\text { Number of bands of adhesions between viscera and } \\
\text { abdominal wall }{ }^{64} \text { (rabbit) }\end{array}$ \\
\hline Rabbit & 6 weeks & $\begin{array}{l}\text { Parietex Composite Ventral Patch } \\
\text { (Covidien) VS Proceed Ventral Patch }\end{array}$ & $\begin{array}{l}\text { Polyester mesh }+ \\
\text { collagen }\end{array}$ & Percentage of adhesion-area ${ }^{57,60}$ \\
\hline Rabbit & 6 weeks & $\begin{array}{l}\text { Parietex Composite Ventral Patch } \\
\text { (Covidien) VS Ventralex ST Hernia Patch } \\
\text { (Bard) }\end{array}$ & No preference & Percentage of adhesion-area ${ }^{57,60}$ \\
\hline
\end{tabular}


Table S8.12 (continued)

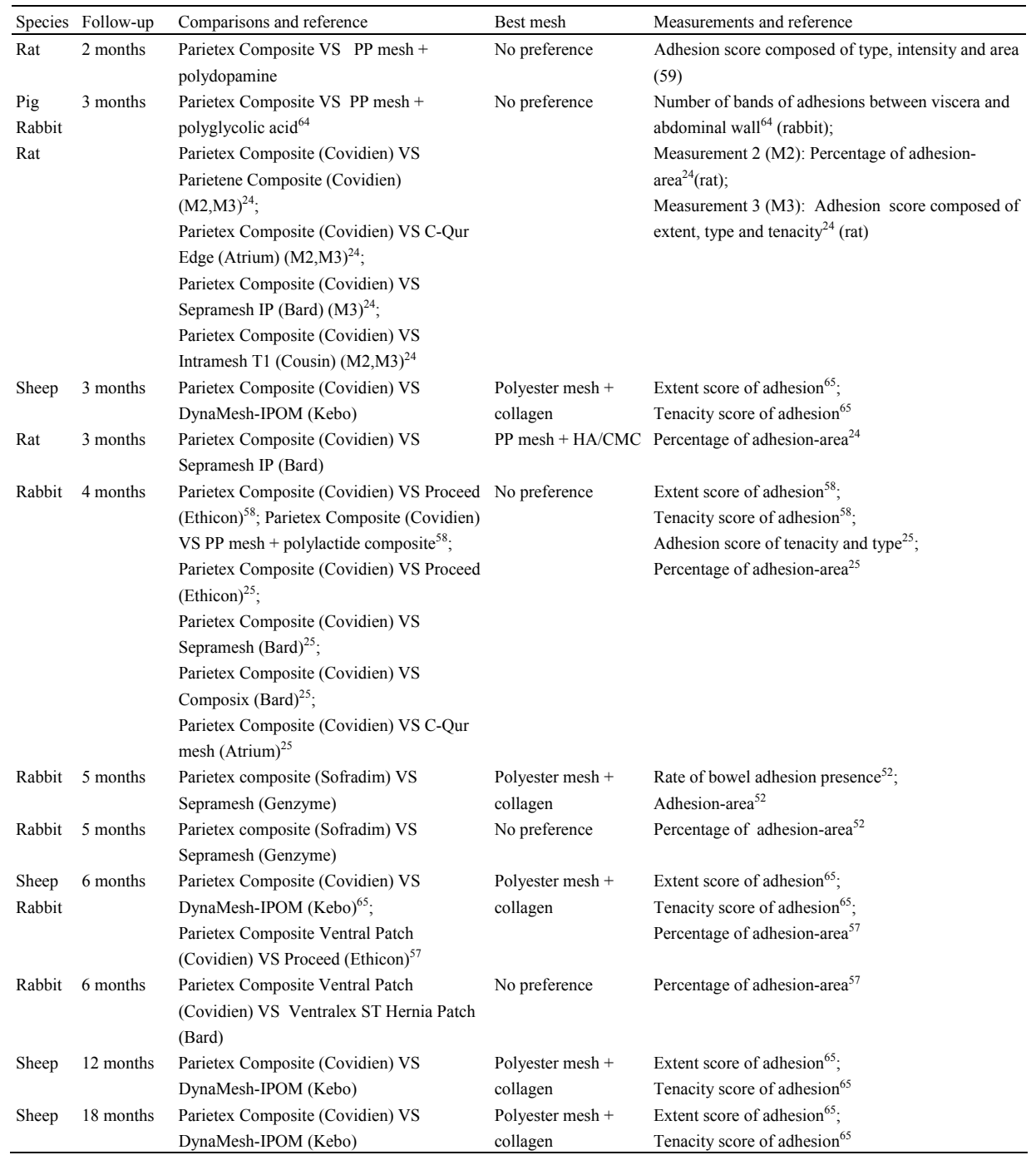




\section{References (for Supplementary Tables S8.2-S8.12)}

1. Alponat A, Lakshminarasappa SR, Teh M, Rajnakova A, Moochhala S, Goh PM, et al. Effects of physical barriers in prevention of adhesions: an incisional hernia model in rats. The Journal of surgical research. 1997;68(2):126-32.

2. Felemovicius I, Bonsack ME, Hagerman G, Delaney JP. Prevention of adhesions to polypropylene mesh. Journal of the American College of Surgeons. 2004;198(4):543-8.

3. Malazgirt Z, Ulusoy AN, Gok Y, Karagoz F, Tac K. Bioabsorbable membrane prevents adhesions to polypropylene mesh in rats. Hernia : the journal of hernias and abdominal wall surgery. 2000;4(3): 129-33.

4. Burger JW, Halm JA, Wijsmuller AR, ten Raa S, Jeekel J. Evaluation of new prosthetic meshes for ventral hernia repair. Surgical endoscopy. 2006;20(8):1320-5.

5. van 't Riet M, de Vos van Steenwijk PJ, Bonthuis F, Marquet RL, Steyerberg EW, Jeekel J, et al. Prevention of adhesion to prosthetic mesh: comparison of different barriers using an incisional hernia model. Annals of surgery. 2003;237(1):123-8.

6. Baptista ML, Bonsack ME, Delaney JP. Seprafilm reduces adhesions to polypropylene mesh. Surgery. 2000;128(1):86-92.

7. Besim H, Yalcin Y, Hamamci O, Arslan K, Sonisik M, Korkmaz A, et al. Prevention of intraabdominal adhesions produced by polypropylene mesh. European surgical research Europaische chirurgische Forschung Recherches chirurgicales europeennes. 2002;34(3):239-43.

8. Ozmen MM, Aslar AK, Terzi MC, Albayrak L, Berberoglu M. Prevention of adhesions by bioresorbable tissue barrier following laparoscopic intraabdominal mesh insertion. Surgical Laparoscopy, Endoscopy and Percutaneous Techniques. 2002;12(5):342-6.

9. Bellon JM, Garcia-Honduvilla N, Serrano N, Rodriguez M, Pascual G, Bujan J. Composite prostheses for the repair of abdominal wall defects: effect of the structure of the adhesion barrier component. Hernia : the journal of hernias and abdominal wall surgery. 2005;9(4):338-43.

10. Szabo A, Haj M, Waxsman I, Eitan A. Evaluation of seprafilm and amniotic membrane as adhesion prophylaxis in mesh repair of abdominal wall hernia in rats. European surgical research Europaische chirurgische Forschung Recherches chirurgicales europeennes. 2000;32(2):125-8.

11. Gonzalez R, Rodeheaver GT, Moody DL, Foresman PA, Ramshaw BJ. Resistance to adhesion formation: a comparative study of treated and untreated mesh products placed in the abdominal cavity. Hernia : the journal of hernias and abdominal wall surgery. 2004;8(3):213-9.

12. Borrazzo EC, Belmont MF, Boffa D, Fowler DL. Effect of prosthetic material on adhesion formation after laparoscopic ventral hernia repair in a porcine model. Hernia : the journal of hernias and abdominal wall surgery. 2004;8(2):108-12.

13. Mayagoitia-Gonzalez JC, Gudino-Amezcua LM, Rivera-Barragan V, Mellado-Diaz AV, Diaz-Chavez EP. Prevention of intestinal adhesions as a result of intraperitoneal mesh with the addition of hyaluronic acid/carboxymethylcellulose gel. Experimental model in rats. Cir Cir. 2012;80(2):150-6.

14. Greenawalt KE, Butler TJ, Rowe EA, Finneral AC, Garlick DS, Burns JW. Evaluation of sepramesh biosurgical composite in a rabbit hernia repair model. The Journal of surgical research. 2000;94(2): 92-8.

15. Dinsmore RC, Calton WC, Jr., Harvey SB, Blaney MW. Prevention of adhesions to polypropylene mesh in a traumatized bowel model. Journal of the American College of Surgeons. 2000;191(2):131-6.

16. Dasiran F, Eryilmaz R, Isik A, Okan I, Somay A, Sahin M. The effect of polyethylene glycol adhesion barrier (Spray Gel) on preventing peritoneal adhesions. Bratislavske lekarske listy. 2015;116(6): 379-82.

17. Dilege E, Coskun H, Gunduz B, Sakiz D, Mihmanli M. Prevention of adhesion to prosthetic mesh in incisional ventral hernias: comparison of different barriers in an experimental model. European surgical research Europaische chirurgische Forschung Recherches chirurgicales europeennes. 2006;38(3):358-64. 
18. Kayaoglu HA, Ozkan N, Hazinedaroglu SM, Ersoy OF, Erkek AB, Koseoglu RD. Comparison of adhesive properties of five different prosthetic materials used in hernioplasty. Journal of investigative surgery : the official journal of the Academy of Surgical Research. 2005;18(2):89-95.

19. Yelimlies B, Alponat A, Cubukcu A, Kuru M, Oz S, Ercin C, et al. Carboxymethylcellulose coated on visceral face of polypropylene mesh prevents adhesion without impairing wound healing in incisional hernia model in rats. Hernia : the journal of hernias and abdominal wall surgery. 2003;7(3):130-3.

20. Kramer K, Senninger N, Herbst H, Probst W. Effective prevention of adhesions with hyaluronate. Archives of surgery (Chicago, Ill : 1960). 2002;137(3):278-82.

21. Erpek H, Tuncyurek P, Soyder A, Boylu S. Hyaluronic acid/carboxymethylcellulose membrane barrier versus taurolidine for the prevention of adhesions to polypropylene mesh. European surgical research Europaische chirurgische Forschung Recherches chirurgicales europeennes. 2006;38(4):414-7.

22. Sikkink CJ, Vries de Reilingh TS, Malyar AW, Jansen JA, Bleichrodt RP, van Goor H. Adhesion formation and reherniation differ between meshes used for abdominal wall reconstruction. Hernia : the journal of hernias and abdominal wall surgery. 2006;10(3):218-22.

23. Nohuz E, Alaboud M, Darcha C, Alloui A, Aublet-Cuvelier B, Jacquetin B. Effectiveness of Hyalobarrier and Seprafilm to prevent polypropylene mesh shrinkage: a macroscopic and histological experimental study. International urogynecology journal. 2014;25(8):1081-7.

24. Schreinemacher MH, van Barneveld KW, Dikmans RE, Gijbels MJ, Greve JW, Bouvy ND. Coated meshes for hernia repair provide comparable intraperitoneal adhesion prevention. Surgical endoscopy. 2013;27(11):4202-9.

25. Pierce RA, Perrone JM, Nimeri A, Sexton JA, Walcutt J, Frisella MM, et al. 120-day comparative analysis of adhesion grade and quantity, mesh contraction, and tissue response to a novel omega-3 fatty acid bioabsorbable barrier macroporous mesh after intraperitoneal placement. Surgical innovation. 2009;16(1):46-54.

26. Schreinemacher MH, Emans PJ, Gijbels MJ, Greve JW, Beets GL, Bouvy ND. Degradation of mesh coatings and intraperitoneal adhesion formation in an experimental model. The British journal of surgery. 2009;96(3):305-13.

27. Bellon JM, Rodriguez M, Garcia-Honduvilla N, Pascual G, Gil VG, Bujan J. Peritoneal effects of prosthetic meshes used to repair abdominal wall defects: monitoring adhesions by sequential laparoscopy. Journal of Laparoendoscopic and Advanced Surgical Techniques. 2007;17(2):160-6.

28. Bellon JM, Garcia-Carranza A, Jurado F, Garcia-Honduvilla N, Carrera-San Martin A, Bujan J. Peritoneal regeneration after implant of a composite prosthesis in the abdominal wall. World journal of surgery. 2001;25(2):147-52.

29. Lamber B, Grossi JV, Manna BB, Montes JH, Bigolin AV, Cavazzola LT. May polyester with collagen coating mesh decrease the rate of intraperitoneal adhesions in incisional hernia repair? Arquivos brasileiros de cirurgia digestiva : $\mathrm{ABCD}=$ Brazilian archives of digestive surgery. 2013;26(1):13-7.

30. Biondo-Simoes ML, Carvalho LB, Conceicao LT, Santos KB, Schiel WA, Arantes M, et al. Comparative study of Polypropylene versus Parietex composite(R), Vicryl(R) and Ultrapro(R) meshes, regarding the formation of intraperitoneal adhesions. Acta cirurgica brasileira. 2017;32(2):98-107.

31. Voskerician G, Jin J, White MF, Williams CP, Rosen MJ. Effect of biomaterial design criteria on the performance of surgical meshes for abdominal hernia repair: a pre-clinical evaluation in a chronic rat model. Journal of materials science Materials in medicine. 2010;21(6):1989-95.

32. Ditzel M, Deerenberg EB, Grotenhuis N, Harlaar JJ, Monkhorst K, Bastiaansen-Jenniskens YM, et al. Biologic meshes are not superior to synthetic meshes in ventral hernia repair: an experimental study with long-term follow-up evaluation. Surgical endoscopy. 2013;27(10):3654-62.

33. van't Riet M, Burger JW, Bonthuis F, Jeekel J, Bonjer HJ. Prevention of adhesion formation to polypropylene mesh by collagen coating: a randomized controlled study in a rat model of ventral hernia repair. Surgical endoscopy. 2004;18(4):681-5.

34. Aliabadi-Wahle S, Choe EU, Jacob-LaBarre J, Flint LM, Ferrara JJ. Evaluation of a novel synthetic material for closure of large abdominal wall defects. Surgery. 1996;119(2):141-5. 
35. Bellon JM, Garcia-Carranza A, Jurado F, Garcia-Honduvilla N, Carrera-San Martin A, Bujan J. Evaluation of a new composite prosthesis (PL-PU99) for the repair of abdominal wall defects in terms of behavior at the peritoneal interface. World journal of surgery. 2002;26(6):661-6.

36. Ansaloni L, Catena F, Coccolini F, Fini M, Gazzotti F, Giardino R, et al. Peritoneal adhesions to prosthetic materials: an experimental comparative study of treated and untreated polypropylene meshes placed in the abdominal cavity. Journal of laparoendoscopic \& advanced surgical techniques Part A. 2009;19(3):369-74.

37. Emans PJ, Schreinemacher MH, Gijbels MJ, Beets GL, Greve JW, Koole LH, et al. Polypropylene meshes to prevent abdominal herniation. Can stable coatings prevent adhesions in the long term? Annals of biomedical engineering. 2009;37(2):410-8.

38. Demir U, Mihmanli M, Coskun H, Dilege E, Kalyoncu A, Altinli E, et al. Comparison of prosthetic materials in incisional hernia repair. Surgery today. 2005;35(3):223-7.

39. Fitzgibbons Jr RJ, Salerno GM, Filipi CJ, Hunter WJ, Watson P. A laparoscopic intraperitoneal onlay mesh technique for the repair of an indirect inguinal hernia. Annals of surgery. 1994;219(2):144-56.

40. Aramayo AL, Lopes Filho Gde J, Barbosa Cde A, Amaral Vda F, Costa LA. Abdominal wall healing in incisional hernia using different biomaterials in rabbits. Acta cirurgica brasileira. 2013;28(4):307-16.

41. Rossi LF, Trindade MRM, AJ DA, Meurer L. PERITONEAL ADHESIONS TYPE I, III AND TOTAL COLLAGEN ON POLYPROPYLENE AND COATED POLYPROPYLENE MESHES: EXPERIMENTAL STUDY IN RATS. Arquivos brasileiros de cirurgia digestiva : ABCD = Brazilian archives of digestive surgery. 2017;30(2):77-82.

42. Novitsky YW, Harrell AG, Cristiano JA, Paton BL, Norton HJ, Peindl RD, et al. Comparative evaluation of adhesion formation, strength of ingrowth, and textile properties of prosthetic meshes after long-term intra-abdominal implantation in a rabbit. The Journal of surgical research. 2007;140(1):6-11.

43. Bellon JM, Rodriguez M, Garcia-Honduvilla N, Gomez-Gil V, Pascual G, Bujan J. Postimplant behavior of lightweight polypropylene meshes in an experimental model of abdominal hernia. Journal of Investigative Surgery. 2008;21(5):280-7.

44. de Vries Reilingh TS, van Goor H, Koppe MJ, Bodegom ME, Hendriks T, Bleichrodt RP. Interposition of Polyglactin Mesh Does Not Prevent Adhesion Formation Between Viscera and Polypropylene Mesh. Journal of Surgical Research. 2007;140(1):27-30.

45. Goldenberg A, Matone J, Marcondes W, Herbella FA, Farah JF. Comparative study of inflammatory response and adhesions formation after fixation of different meshes for inguinal hernia repair in rabbits. Acta cirurgica brasileira / Sociedade Brasileira para Desenvolvimento Pesquisa em Cirurgia. 2005;20(5):347-52.

46. Liu L, Petro C, Majumder A, Fayezizadeh M, Anderson J, Novitsky YW. The use of Vicryl mesh in a porcine model to assess its safety as an adjunct to posterior fascial closure during retromuscular mesh placement. Hernia : the journal of hernias and abdominal wall surgery. 2016;20(2):289-95.

47. Schug-Pass C, Sommerer F, Tannapfel A, Lippert H, Kockerling F. The use of composite meshes in laparoscopic repair of abdominal wall hernias: are there differences in biocompatibily?: experimental results obtained in a laparoscopic porcine model. Surgical endoscopy. 2009;23(3):487-95.

48. Bellon JM, Rodriguez M, Garcia-Honduvilla N, Gomez-Gil V, Pascual G, Bujan J. Real-time monitoring of the peritoneal behavior of composite prostheses by sequential laparoscopy: Applicability in ventral hernia repair. [Spanish]. Cirugia espanola. 2007;82(5):290-6.

49. Rodriguez M, Pascual G, Sotomayor S, Perez-Koihler B, Cifuentes A, Bellon JM. Chemical adhesion barriers: Do they affect the intraperitoneal behavior of a composite mesh? Journal of Investigative Surgery. 2011;24(3):115-22.

50. Konerding MA, Chantereau P, Delventhal V, Holste JL, Ackermann M. Biomechanical and histological evaluation of abdominal wall compliance with intraperitoneal onlay mesh implants in rabbits: a comparison of six different state-of-the-art meshes. Medical engineering \& physics. 2012;34(7):806-16.

51. Matthews BD, Mostafa G, Carbonell AM, Joels CS, Kercher KW, Austin C, et al. Evaluation of adhesion formation and host tissue response to intra-abdominal polytetrafluoroethylene mesh and composite prosthetic mesh. Journal of Surgical Research. 2005;123(2):227-34. 
52. Johnson EK, Hoyt CH, Dinsmore RC. Abdominal wall hernia repair: a long-term comparison of Sepramesh and Dualmesh in a rabbit hernia model. The American surgeon. 2004;70(8):657-61.

53. Deeken CR, Matthews BD. Comparison of contracture, adhesion, tissue ingrowth, and histologic response characteristics of permanent and absorbable barrier meshes in a porcine model of laparoscopic ventral hernia repair. Hernia : the journal of hernias and abdominal wall surgery. 2011: $1-8$.

54. Gruber-Blum S, Petter-Puchner AH, Brand J, Fortelny RH, Walder N, Oehlinger W, et al. Comparison of three separate antiadhesive barriers for intraperitoneal onlay mesh hernia repair in an experimental model. The British journal of surgery. 2011;98(3):442-9.

55. Leblebici IM, Bozkurt S, Sever B. Comparison of adhesion-preventing meshes in a rat model of incisional hernia in terms of intra-abdominal adhesions and other complications. Biomedical Research (India). 2014;25(3):414-9.

56. Celik A, Altinli E, Koksal N, Celik AS, Onur E, Ozkan OF, et al. The shrinking rates of different meshes placed intraperitoneally: a long-term comparison of the TiMesh, VYPRO II, Sepramesh, and DynaMesh. Surgical laparoscopy, endoscopy \& percutaneous techniques. 2009;19(4):e130-4.

57. Garcia-Moreno F, Sotomayor S, Perez-Lopez P, Perez-Kohler B, Bayon Y, Pascual G, et al. Intraperitoneal behaviour of a new composite mesh (Parietex Composite Ventral Patch) designed for umbilical or epigastric hernia repair. Surgical endoscopy. 2014;28(12):3479-88.

58. Dolce CJ, Keller JE, Stefanidis D, Walters KC, Heath JJ, Lincourt AL, et al. Evaluation of soft tissue attachments to a novel intra-abdominal prosthetic in a rabbit model. Surgical innovation. 2012;19(3):295-300.

59. Hu M, Lin X, Huang R, Yang K, Liang Y, Zhang X, et al. Lightweight, Highly Permeable, Biocompatible, and Antiadhesive Composite Meshes for Intraperitoneal Repairs. Macromolecular bioscience. 2018;18(7):e1800067.

60. Garcia-Moreno F, Perez-Lopez P, Sotomayor S, Perez-Kohler B, Bayon Y, Pascual G, et al. Comparing the host tissue response and peritoneal behavior of composite meshes used for ventral hernia repair. The Journal of surgical research. 2015;193(1):470-82.

61. Bellon JM, Garcia-Honduvilla N, Jurado F, Garcia-Carranza A, Garcia-Moreno F, Martin AC, et al. Use of composite prostheses in the repair of defects in the abdominal wall: prosthetic behaviour at the peritoneum. The European journal of surgery = Acta chirurgica. 2001;167(9):666-71.

62. Duffy AJ, Hogle NJ, LaPerle KM, Fowler DL. Comparison of two composite meshes using two fixation devices in a porcine laparoscopic ventral hernia repair model. Hernia : the journal of hernias and abdominal wall surgery. 2004;8(4):358-64.

63. Jacob BP, Hogle NJ, Durak E, Kim T, Fowler DL. Tissue ingrowth and bowel adhesion formation in an animal comparative study: polypropylene versus Proceed versus Parietex Composite. Surgical endoscopy. 2007;21(4):629-33.

64. de Araujo UR, Czeczko NG, Ribas-Filho JM, Malafaia O, Budel VM, Balderrama CM, et al. Intraperitoneal meshes in the repair of abdominal wall defects: comparison of polyester with collagen versus polypropylene with polyglycolic acid. Revista do Colegio Brasileiro de Cirurgioes. 2009;36(3):241-9.

65. Zinther NB, Wara P, Friis-Andersen H. Intraperitoneal onlay mesh: an experimental study of adhesion formation in a sheep model. Hernia : the journal of hernias and abdominal wall surgery. 2010;14(3):283-9. 

Part IV

Discussion and summary 



\section{Chapter 9}

General discussion and future perspectives 
Chapter 9 
Incisional hernias and abdominal adhesions are two of the most common complications postsurgery linked to tissue healing. ${ }^{1,2}$ Tissue healing is a dynamic and complex process which entails regenerating or repairing damaged tissues in response to injury. Different tissue types or differently-located tissues have unequal healing rate even following an identical surgical procedure. ${ }^{3,4}$ The underlying cellular and molecular events of healing vary in specific tissues despite the fact that virtually all types of soft tissues have comparable healing stages. A better understanding of the healing process in specific tissue could lead to therapies that prevent healing failure or speed up normal healing. Normal fascial healing is essential to avoid the formation of incisional hernia. In this thesis, we examined the cellular and molecular events in normal fascial healing in the early postoperative period. Moreover, we investigated novel physical barriers that could prevent the formation of abdominal adhesions in the form of excessive tissue restoration.

\section{Part I The ideal animal model}

Animal experiments play a significant role in filling up knowledge gaps in human disease and health. High reporting quality of animal studies is necessary for the reproducibility of animal experiments and to avoid unnecessary animal studies. ${ }^{5}$ ARRIVE guidelines (Animal Research: Reporting of In Vivo Experiments) have been developed to maximise the reporting information and improve the reporting quality of animal experiments. They were first published in $2010,{ }^{6}$ and updated in 2019 and formally published in 2020. ${ }^{7}$ Chapter 2 investigated the effect of ARRIVE guidelines on reporting quality of animal experiments using hernia models repaired with meshes. Comparisons were performed between articles published five years before ARRIVE2010 guidelines (from July of 2005 to June of 2010; pre-ARRIVE) and articles published within five years until the updated ARRIVE-2020 (from July of 2014 to June of 2019; post-ARRIVE), using the original ARRIVE-2010 guidelines. Articles published last year (from July of 2019 to July of 2020) were evaluated using ARRIVE2020 guidelines as criteria for presenting the most recent reporting quality. We found that, compared to pre-ARRIVE, mean numbers of fully-reported (sub)items per article significantly increased post-ARRIVE for all species. However, the mean numbers of fully-reported (sub)items per article post-ARRIVE were only 14 out of 35 (sub)items that should be reported according to ARRIVE checklists. Species-specific analysis revealed a significant increase of mean numbers of fully-reported items/subitems per article in rabbit studies and pig studies, but not in rat studies. Every (sub)item of ARRIVE-2010 was also compared in studies between pre-ARRIVE and post-ARRIVE for overall species, rats, rabbits, and pigs. A few of (sub)items with significant improvements post-ARRIVE were distributed unequally among specific species. For 
example, the item Experimental procedures (subitem 7a in ARRIVE-2010 and essential item 9 in ARRIVE-2020, more specifically anaethesia, analgesia, surgical procedure, euthanasia, and interventions, has been improved significantly for post-ARRIVE overall. The improvement of this subitem was however only found in studies using rats and rabbit species but not in pig studies. Despite these improvements, a large number of (sub)items remain reported poorly. For instance, essential subitems, concerning time, location and rationale of experimental procedures, animal details, sample size calculation, allocation details, units for and assumptions of statistical analysis, and a measure of precision, appeared not significantly improved overall. Therefore, on the one hand, the ARRIVE-2010 guidelines have a positive effect on improving the reporting quality of animal experiments, although an imbalance is observed between the different animal species. On the other hand, many (sub)items remain un(der)reported, leaving room for the improvement for future animal studies.

The sex of animals is an important variable to take into account when designing and analysing preclinical studies. ${ }^{8,9}$ Chapter 3 shows the difference in adhesion formation between male and female rats in an ischemic button model. In this chapter, the difference in abdominal wall healing between both sexes is also investigated due to the fact that the ischaemic button model established via a midline laparotomy. Both qualitative and quantitative adhesion scores were determined with no significant differences between male and female rats. Despite the fact that two males had wound dehiscence, the underlying fascia remained intact. Moreover, no significant sex-related difference was identified regarding the wound score. This indicates that there is macroscopically no significant difference between sexes regarding the intraperitoneal adhesion formation to the ischaemic button as well as the fascial healing in abdominal wall. However, compared to female rats, male rats gained weight faster and reached the baseline weight sooner during recovery. Multiple factors may explain the faster weight gain in male rats, for example, recovery rate of different sex, insufficient analgesia, or unrecognised need for pain relief in female groups. ${ }^{10}$

\section{PART II Repair of fascia in the abdominal wall}

Normal fascial healing is of importance to restore the integrity of abdominal wall. Early mechanical failure of fascia can result in the formation of incisional hernia. ${ }^{11}$ However, scarce studies concern the normal healing of acutely injured fascia, especially in the early period postsurgery. ${ }^{12}$

Cellular activities, such as migration, proliferation, and differentiation are essential to normal tissue healing. The temporal changes of cellular population and gene expression 
in fascial healing are described in chapter 4. Granulocytes, macrophages, and fibroblasts were evaluated microscopically using a histological scoring system. The scores of granulocytes and macrophages were significantly changed over time. The related gene expression levels of these cells were consistent with the histological scores. Additionally, the cellular dominations in rats in this study seem comparable to previous studies in guinea pigs. ${ }^{13}$ These findings indicate that granulocytes predominate in the inflammatory cells of fascia in the first postoperative days and are then replaced by the macrophages. We also found that fibroblasts began to appear at three days postsurgery and reached the highest level at seven days postsurgery. This suggests that fibroblasts may play an important role at the later stages of fascia healing.

Previous studies have illustrated that macrophage polarization is involved in the inflammatory response to acute skin healing. ${ }^{14,15}$ Classically activated macrophages (M1-like phenotypes) present pro-inflammatory function whereas alternatively activated macrophages (M2-like phenotypes) inhibit the inflammatory response and promote regeneration. The balance of M1 and M2 is a vital mechanism for normal wound healing. Our study demonstrated that in fascial healing, M1-related gene levels reached the highest level at six hours and decreased afterwards. M2-related gene levels increased to the highest level at three days. This is an indication that macrophages predominate from M1-like phenotypes to M2-like phenotypes in fascial healing within three days postsurgery. It should be noted that there is no absolute separation between the two macrophage phenotypes ${ }^{16}$ and that several subpopulations of macrophages have been described. ${ }^{17,18}$ Therefore, M1/M2 polarization in vivo may reflect a more complex response than initially thought. The domination of inflammatory cells between fascial healing and skin wound healing seems comparable based on our results.

However, previous studies have demonstrated that the healing rate in acutely injured fascia differs from skin. ${ }^{19,20}$ Furthermore, $T g f b l$ expression level, playing an important role in wound healing, seems different between fascial and skin wound healing. Overexpression or deficiency of $T g f b l$ may lead to a delay of full-thickness wound healing ${ }^{21,22}$ while overexpression of $T g f b l$ accelerates superficial wound healing (epidermis only). In deep skin wound, the $T g f b l$ expression level rapidly increased after injury and reached a peak at 3 days post-injury (21). Our results on fascial healing showed that rats healed in 7 days and $T g f b 1$ expression level did not change significantly during this period, indicating a stable level of $T g f b l$ expression level in normal fascial healing. This suggests that fascial healing differs from skin wound healing on gene expression level.

Lipids, as compounds of signalling and structural membrane of cells, play an important role of healing process. Lipid mediators, derived from fatty acids such as arachidonic, ${ }^{23}$ eicosapentaenoic, or docosahexaenoic acids, ${ }^{24}$ may affect skin wound healing. Omega- 
6 fatty acids can modulate cell migration and proliferation during the healing process. ${ }^{25}$ Chapter 5 focuses on evaluating the temporal changes and spatial distribution of lipids and their correlation with the acute inflammatory response and fibroblasts in fascial healing. Matrix - assisted laser desorption/ionization (MALDI) combined with time - of - flight mass spectrometry (TOF-MS) was used to analyse lipids directly from their native tissues in a label free way. Linear regression analysis was done with $\mathrm{R}$ to model the relationship between the intensity of mass to charge $(\mathrm{m} / \mathrm{z})$ values and healing time. A total of 18 different mass to charge $(\mathrm{m} / \mathrm{z})$ values for positive ionization mode and $35 \mathrm{~m} / \mathrm{z}$ values for negative ionization mode were found with significance over time using the linear regression analysis. From these $53 \mathrm{~m} / \mathrm{z}$ signals, we identified five different lipid species belonging to phosphatidylcholine (PC), phosphatidylethanolamine (PE), and monosialodihexosylganglioside (GM) that correlated with histological scores. These lipid species could be biomarkers for inflammatory cells and fibroblasts in fascial healing based on the correlation found in our study. One PE (PE O-36:5) and two PC lipid species (PC O-38:5 and PC 38:4) were linked with inflammation score. PC 32:0 and GM3 34:1;2 were correlated with fibroblast growth. PCs are a class of phospholipids with choline attached as a head group and have effects on wound healing process, however, the effect remains controversial. ${ }^{26-28} \mathrm{PEs}$ can be converted to PCs by methylations via catalyzation and are hence involved in the biogenesis of PCs. ${ }^{29,30}$ Therefore, PE O-36:5, PC O-38:5, PC 38:4 and PC 32:0 could be involved in abdominal fascial healing. Previous studies have illustrated that exogenetic PCs may promote mucosal healing and surgical wound healing. ${ }^{26,31,32}$ PCs and Pes, as found in our study, could be potential therapeutics in high-risk IH patients. Additionally, PC 32:0 and GM3 34:1;2 were significantly increased in normal healing and corresponded to fibroblasts growth, indicating they could be promoters of fibroblasts. However, GM3 belonging to gangliosides is observed to be a driver of impaired wound healing and depletion of GM3 can lead to improvement of wound healing in diabetic mice. ${ }^{33,34}$ This is inconsistent with our findings and could be explained by a difference in molecules in fascial healing and skin wound healing.

\section{PART III Prevention of intraperitoneal adhesions}

Abdominal adhesions occur in virtually all patients after laparotomy, leading to complications commonly known as pain, intestinal obstruction, female infertility, and difficulties during reoperation. Application of physical barriers is one of the most promising strategies for reducing abdominal adhesions, by preventing the direct contact between the surfaces of two traumatized tissues before they have a chance to stick to each other. ${ }^{35}$ Physical barriers consisting of dissolvable polymers have a significant 
impact on preventing the intraperitoneal adhesion formation. ${ }^{36}$ However, there is still room for improvement to develop novel polymer materials due to insufficient antiadhesive efficacy or other drawbacks of these barriers, such as poor wound healing, haemorrhage, inconvenient application during surgery, high costs, and the lack of convincing evidence. ${ }^{35,37}$ Currently, various types of antiadhesive barriers are commercially available or under laboratory testing, in the forms of films, gels, sprays, and fluids.

In order to evaluate the antiadhesive efficacy of barriers, Chapter 6 compared five new absorbable films in an adhesion rat model using peritoneal ischemic button technique. A previous study has demonstrated that the peritoneal ischemic button model is the most consistent and reproducible adhesion model, compared to parietal peritoneum excision models, peritoneum abrasion models, or cecal abrasion models. ${ }^{38} \mathrm{We}$ found that, in this model, the median number of buttons with adhesions in control animals was eight of eight buttons. This suggests that the peritoneal ischemic button technique is sufficient to induce the intraperitoneal adhesions in rats.

Nair's score and Zühlke score, referring to the quantity and quality of adhesions, ${ }^{39,40}$ respectively, were used to measure the antiadhesive efficacy of the different barriers in our study. No statistical differences were found between any of the five polymer films when using the two scoring systems. However, a significant difference between groups was found when counting the numbers of buttons involved in adhesions. Counting the numbers of buttons with adhesions could be a better method to evaluate intraperitoneal adhesions for this particular model. Among the five new polymer antiadhesive barriers, one barrier displayed a significantly lower number of buttons with adhesions. However, this barrier resulted in more complications than in other groups. Therefore, application of that barrier should be evaluated further before application in humans if possible.

Chapter 7 compared a novel hyaluronic acid gel to a commercially available antiadhesive gel, hyaluronate carboxymethyl cellulose (HA-CMC) gel, measuring the antiadhesive efficacy in the button-adhesion model. Macroscopic adhesions were measured by Nair's core, Zühlke score, vascularisation, number of buttons involved in adhesions, and number of organs involved in adhesions. Only the number of involved organs was reduced significantly for the hyaluronic acid gel compared to the HA-CMC gel. In order to gain more pathophysiological insight into the adhesion development, gene expression levels were compared between buttons with adhesions and buttons without adhesions. Two typical M2 makers, Clec $10 a$ and $C d 163$, were significantly higher expressed in buttons with adhesions compared to buttons without adhesions two weeks postoperatively. The prolonged M2 activation may be explained because of extended remodelling in the injured tissue. It is also possible that the prolonged M2 activation is correlated with the pathophysiological process of adhesion formation. 
Persistent activation of M2 macrophages can impair normal tissue healing and can lead to activation of fibrotic process. ${ }^{41}$ Blocking the persistent activation could be a strategy to prevent adhesion formation. It is important to mention that concerns remain regarding whether or not antiadhesive barriers have a negative effect on the healing of intestinal anastomosis. ${ }^{37,42}$

Intraperitoneal onlay meshes are widely applied in hernia repair. Adhesions can however form between internal abdominal tissues to the visceral surface of the mesh. ${ }^{43}$ Coatings on the mesh surface, as a protective layer separating the internal tissues and the mesh, have been introduced to prevent the adhesion-to-mesh formation. ${ }^{44}$ Due to the limitations of clinical studies such as complex regulatory requirements, multiple ethics approvals, slow enrolment, high costs, limited applicability and limited followup time, ${ }^{45,46}$ the evaluation of the antiadhesive efficacy of coated meshes is advisable in animal models prior to the application in humans. ${ }^{47,48}$ There are numerous animal experiments that investigated the antiadhesive characteristics of mesh coatings. In order to summarize these results, Chapter 8 presents a systematic review and meta-analysis aiming to evaluate the antiadhesive efficacy of the mesh coatings in animals.

Compared to polypropylene meshes (PP), PP meshes coated with hyaluronic acid/carboxymethyl cellulose (HA/CMC) were found to significantly reduce intraperitoneal adhesion formation. Heterogeneity was low for this comparison, indicating the consistent antiadhesive effect of HA/CMC-coatings on PP meshes in animal studies. Polyester meshes coated with collagen were not significantly superior to PP meshes in the meta-analysis. However, high heterogeneity for this comparison was identified despite mesh characteristics, follow-up, species, and fixation sutures for meshes being virtually identical. Sources for this heterogeneity are complicated to investigate due to the lack of details regarding allocation details of animals, housing conditions for animals, details of microbiomes, as well as unknown factors that might contribute to the heterogeneity.

Despite hundreds of animal experiments that were eligible for inclusion in the systematic review and meta-analysis, only several studies could finally be included for meta-analysis. This is mainly because of the limited availability of animal studies with an identical comparison between two mesh coatings in the same conditions. Many factors may contribute to this. Firstly, characteristics of a mesh, regarding mesh coating, material absorption, pore size, weight, constitution, knitting structure, and fixation may influence the adhesion-to-mesh formation. ${ }^{44}$ Secondly, there were a large number of scoring systems evaluating the quality and quantity of adhesion formation. No standard scoring systems were widely accepted for the adhesion evaluation in animal models. Lastly, animal models with various species, as well as techniques of adhesion establishment, were presented differently mimicking adhesion formation in 
humans. Therefore, a standard adhesion scoring system and an optimal animal model that can be widely accepted are urgently required for further animal studies assessing the antiadhesive efficacy of mesh coatings. Recently, a MEsh Tissue Adhesion scoring system (META score) has been published with a wide consensus of renowned experts. ${ }^{49}$ This scoring system can be used for the evaluation of mesh-related adhesions and could help improve comparability of further studies concerning mesh-related adhesions.

\section{The perspective of future}

Incisional hernias remain a frequent complication despite optimized fascial closure techniques and prophylactic mesh placements in high-risk patients, which have significantly reduced the incidence of incisional hernia. ${ }^{50-52}$ Failed healing of abdominal fascia due to early mechanical failure leads to the formation of incisional hernia. ${ }^{11,53}$ However, the pathophysiological knowledge of the fascial healing remains limited. The functional role of involved cellular and molecular events in the fascial healing needs further investigation. The better understanding of these events, the more likely we are to develop effective strategies for preventing and treating incisional hernia.

In our study, macrophage polarisation was discovered in fascial healing. Future studies establishing the role of macrophages in normal and failed fascial healing are promising; macrophages could become a therapeutic target in avoiding the formation of incisional hernia.

Time-dependent alterations in lipid species were found in our studies following acute injury of the abdominal fascia. Various types of lipid species have been investigated with different impacts on skin wound healing. ${ }^{54-57}$ Therefore, applications of novel lipid compounds that could promote fascial healing may be a promising approach to prevent incisional hernia. Besides lipids, growth factors were shown to have a positive effect on fascial healing in animal models. ${ }^{58-60}$ Identifying molecular compounds that could promote fascial healing should be high priority in future studies. In that context, protein profiling in fascial healing could be of interest for future studies. For instance, it has

been described that abnormal collagen synthesis could lead to IH formation. ${ }^{61,62}$ In addition to collagen, other proteins could also play a role, which needs to be further investigated.

Intraperitoneal onlay mesh placement has a clinical role in the repair of incisional hernia. ${ }^{63}$ Preventing the formation of the tissue-to-mesh adhesion is a challenge for the 
application of onlay meshes. Novel antiadhesive barriers with the more cost-effective and less negative influence of anastomotic healing should continue to receive attention in future studies. In the meantime, surgical hernia repair should be performed in a way that intraperitoneal meshes are avoided if possible ${ }^{50,63,64}$ since the ideal antiadhesive mesh is yet to be invented.

The efficacy of antiadhesive coatings is often tested in in vivo animal experiments prior to human use. However, standard adhesion evaluation systems matching specific animal models were lacking, which inhibits the comparative evaluations of different coating barriers. The META score has been published, which addresses this problem. Further studies concerning mesh-related adhesions should use this scoring system to minimize measurement variability between studies.

Animal experiments remain a critical role in biomedical studies. The 3Rs (Replacement, Reduction, Refinement) principle has been applied in animal testing for several decades. Future studies improving the design, conduct, analysis, and reporting of animal experiments are always required as part of a 3 Rs initiative. 


\section{References}

1. Lindmark M, Strigard K, Lowenmark T, Dahlstrand U, Gunnarsson U. Risk Factors for Surgical Complications in Ventral Hernia Repair. World J Surg. 2018;42(11):3528-36.

2. Okabayashi K, Ashrafian H, Zacharakis E, Hasegawa H, Kitagawa Y, Athanasiou T, et al. Adhesions after abdominal surgery: a systematic review of the incidence, distribution and severity. Surg Today. 2014;44(3):405-20.

3. Stubsgaard AJ, Andresen K, Rosenberg J. [The optimal timing of suture removal depends on the anatomical location]. Ugeskr Laeger. 2015;177(45):V05150390.

4. Thornton FJ, Barbul A. Healing in the gastrointestinal tract. Surg Clin North Am. 1997;77(3):549-73.

5. Kilkenny C, Browne WJ, Cuthill IC, Emerson M, Altman DG. Improving bioscience research reporting: the ARRIVE guidelines for reporting animal research. Osteoarthritis Cartilage. 2012;20(4):256-60.

6. Kilkenny C, Browne WJ, Cuthill IC, Emerson M, Altman DG. Improving bioscience research reporting: the ARRIVE guidelines for reporting animal research. PLoS Biol. 2010;8(6):e1000412.

7. Percie du Sert N, Hurst V, Ahluwalia A, Alam S, Avey MT, Baker M, et al. The ARRIVE guidelines 2.0: Updated guidelines for reporting animal research. PLoS Biol. 2020;18(7):e3000410.

8. Miller LR, Marks C, Becker JB, Hurn PD, Chen WJ, Woodruff T, et al. Considering sex as a biological variable in preclinical research. FASEB J. 2017;31(1):29-34.

9. Lee SK. Sex as an important biological variable in biomedical research. BMB Rep. 2018;51(4):167-73.

10. Waite ME, Tomkovich A, Quinn TL, Schumann AP, Dewberry LS, Totsch SK, et al. Efficacy of Common Analgesics for Postsurgical Pain in Rats. J Am Assoc Lab Anim Sci. 2015;54(4):420-5.

11. Xing L, Culbertson EJ, Wen Y, Franz MG. Early laparotomy wound failure as the mechanism for incisional hernia formation. J Surg Res. 2013;182(1):e35-42.

12. Lau FH, Pomahac B. Wound healing in acutely injured fascia. Wound Repair Regen. 2014;22 Suppl 1:14-7.

13. Ross R, Benditt EP. Wound healing and collagen formation. I. Sequential changes in components of guinea pig skin wounds observed in the electron microscope. J Biophys Biochem Cytol. 1961;11:677700 .

14. Krzyszczyk P, Schloss R, Palmer A, Berthiaume F. The Role of Macrophages in Acute and Chronic Wound Healing and Interventions to Promote Pro-wound Healing Phenotypes. Front Physiol. 2018;9:419.

15. Ferrante CJ, Leibovich SJ. Regulation of Macrophage Polarization and Wound Healing. Adv Wound Care (New Rochelle). 2012;1(1):10-6.

16. Murray PJ, Allen JE, Biswas SK, Fisher EA, Gilroy DW, Goerdt S, et al. Macrophage activation and polarization: nomenclature and experimental guidelines. Immunity. 2014;41(1):14-20.

17. Chavez-Galan L, Olleros ML, Vesin D, Garcia I. Much More than M1 and M2 Macrophages, There are also CD169(+) and TCR(+) Macrophages. Front Immunol. 2015;6:263.

18. Allavena P, Sica A, Garlanda C, Mantovani A. The Yin-Yang of tumor-associated macrophages in neoplastic progression and immune surveillance. Immunol Rev. 2008;222:155-61.

19. Franz MG, Smith PD, Wachtel TL, Wright TE, Kuhn MA, Ko F, et al. Fascial incisions heal faster than skin: a new model of abdominal wall repair. Surgery. 2001;129(2):203-8.

20. Lemonnier F, Gautier M, Wolfrom C, Lemonnier A. Some metabolic differences between human skin and aponeurosis fibroblasts in culture. J Cell Physiol. 1980;104(3):415-23.

21. Wang XJ, Han G, Owens P, Siddiqui Y, Li AG. Role of TGF beta-mediated inflammation in cutaneous wound healing. J Investig Dermatol Symp Proc. 2006;11(1):112-7.

22. Pakyari M, Farrokhi A, Maharlooei MK, Ghahary A. Critical Role of Transforming Growth Factor Beta in Different Phases of Wound Healing. Adv Wound Care (New Rochelle). 2013;2(5):215-24.

23. Tallima H, El Ridi R. Arachidonic acid: Physiological roles and potential health benefits - A review. J Adv Res. 2018;11:33-41.

24. McDaniel JC, Belury M, Ahijevych K, Blakely W. Omega-3 fatty acids effect on wound healing. Wound Repair Regen. 2008;16(3):337-45. 
25. Silva JR, Burger B, Kuhl CMC, Candreva T, Dos Anjos MBP, Rodrigues HG. Wound Healing and Omega-6 Fatty Acids: From Inflammation to Repair. Mediators Inflamm. 2018;2018:2503950.

26. Kornilova ZK, Selishcheva AA, Perel'man MI. Effect of phosphatidylcholine liposome on regeneration of surgical wound in guinea pig lung. Bull Exp Biol Med. 2001;131(2):191-4.

27. Kurinets A, Lichtenberger LM. Phosphatidylcholine-associated aspirin accelerates healing of gastric ulcers in rats. Dig Dis Sci. 1998;43(4):786-90.

28. Burger B, Kuhl CMC, Candreva T, Cardoso RDS, Silva JR, Castelucci BG, et al. Oral administration of EPA-rich oil impairs collagen reorganization due to elevated production of IL-10 during skin wound healing in mice. Sci Rep. 2019;9(1):9119.

29. Becker T, Horvath SE, Bottinger L, Gebert N, Daum G, Pfanner N. Role of phosphatidylethanolamine in the biogenesis of mitochondrial outer membrane proteins. J Biol Chem. 2013;288(23):16451-9.

30. Kawalek A, Jagadeesan C, van der Klei IJ. Impaired biosynthesis of the non-bilayer lipids phosphatidylethanolamine or cardiolipin does not affect peroxisome biogenesis and proliferation in Saccharomyces cerevisiae. Biochem Biophys Res Commun. 2016;480(2):228-33.

31. Antonelli E, Villanacci V, Bassotti G. Novel oral-targeted therapies for mucosal healing in ulcerative colitis. World J Gastroenterol. 2018;24(47):5322-30.

32. Karner M, Kocjan A, Stein J, Schreiber S, von Boyen G, Uebel P, et al. First multicenter study of modified release phosphatidylcholine "LT-02" in ulcerative colitis: a randomized, placebo-controlled trial in mesalazine-refractory courses. Am J Gastroenterol. 2014;109(7):1041-51.

33. Dam DHM, Paller AS. Gangliosides in Diabetic Wound Healing. Prog Mol Biol Transl Sci. 2018;156:229-39.

34. Wang XQ, Lee S, Wilson H, Seeger M, Iordanov H, Gatla N, et al. Ganglioside GM3 depletion reverses impaired wound healing in diabetic mice by activating IGF-1 and insulin receptors. J Invest Dermatol. 2014;134(5):1446-55.

35. Li J, Feng X, Liu B, Yu Y, Sun L, Liu T, et al. Polymer materials for prevention of postoperative adhesion. Acta Biomater. 2017;61:21-40.

36. Park H, Baek S, Kang H, Lee D. Biomaterials to Prevent Post-Operative Adhesion. Materials (Basel). 2020;13(14).

37. Diamond MP, Burns EL, Accomando B, Mian S, Holmdahl L. Seprafilm((R)) adhesion barrier: (2) a review of the clinical literature on intraabdominal use. Gynecol Surg. 2012;9(3):247-57.

38. Whang SH, Astudillo JA, Sporn E, Bachman SL, Miedema BW, Davis W, et al. In search of the best peritoneal adhesion model: comparison of different techniques in a rat model. J Surg Res. 2011;167(2):245-50.

39. Nair SK, Bhat IK, Aurora AL. Role of proteolytic enzyme in the prevention of postoperative intraperitoneal adhesions. Arch Surg. 1974;108(6):849-53.

40. Zuhlke HV, Lorenz EM, Straub EM, Savvas V. [Pathophysiology and classification of adhesions]. Langenbecks Arch Chir Suppl II Verh Dtsch Ges Chir. 1990:1009-16.

41. Conway B, Hughes J. Cellular orchestrators of renal fibrosis. QJM. 2012;105(7):611-5.

42. Diamond MP, Burns EL, Accomando B, Mian S, Holmdahl L. Seprafilm(R) adhesion barrier: (1) a review of preclinical, animal, and human investigational studies. Gynecol Surg. 2012;9(3):237-45.

43. Kokotovic D, Bisgaard T, Helgstrand F. Long-term Recurrence and Complications Associated With Elective Incisional Hernia Repair. JAMA. 2016;316(15):1575-82.

44. Baylon K, Rodriguez-Camarillo P, Elias-Zuniga A, Diaz-Elizondo JA, Gilkerson R, Lozano K. Past, Present and Future of Surgical Meshes: A Review. Membranes (Basel). 2017;7(3).

45. Jones CW, Platts-Mills TF. Understanding commonly encountered limitations in clinical research: an emergency medicine resident's perspective. Ann Emerg Med. 2012;59(5):425-31 e11.

46. de la Torre Hernandez JM, Edelman ER. From Nonclinical Research to Clinical Trials and Patientregistries: Challenges and Opportunities in Biomedical Research. Rev Esp Cardiol (Engl Ed). 2017;70(12):1121-33.

47. Bittner R, Bingener-Casey J, Dietz U, Fabian M, Ferzli GS, Fortelny RH, et al. Guidelines for laparoscopic treatment of ventral and incisional abdominal wall hernias (International Endohernia Society (IEHS)-part 1. Surg Endosc. 2014;28(1):2-29. 
48. Schreinemacher M, Henatsch D, van Barneveld K, Bouvy N. The need for standardised animal models and scoring systems in assessing mesh biocompatibility. Hernia. 2010;14(3):335-6.

49. van den Hil LCL, Mommers EHH, Bosmans J, Morales-Conde S, Gomez-Gil V, LeBlanc K, et al. META Score: An International Consensus Scoring System on Mesh-Tissue Adhesions. World J Surg. 2020;44(9):2935-43.

50. Kohler A, Lavanchy JL, Lenoir U, Kurmann A, Candinas D, Beldi G. Effectiveness of Prophylactic Intraperitoneal Mesh Implantation for Prevention of Incisional Hernia in Patients Undergoing Open Abdominal Surgery: A Randomized Clinical Trial. JAMA Surg. 2019;154(2):109-15.

51. Jairam AP, Lopez-Cano M, Garcia-Alamino JM, Pereira JA, Timmermans L, Jeekel J, et al. Prevention of incisional hernia after midline laparotomy with prophylactic mesh reinforcement: a meta-analysis and trial sequential analysis. BJS Open. 2020.

52. Muysoms FE, Antoniou SA, Bury K, Campanelli G, Conze J, Cuccurullo D, et al. European Hernia Society guidelines on the closure of abdominal wall incisions. Hernia. 2015;19(1):1-24.

53. Pollock AV, Evans M. Early prediction of late incisional hernias. Br J Surg. 1989;76(9):953-4.

54. Kim BE, Goleva E, Hall CF, Park SH, Lee UH, Brauweiler AM, et al. Skin Wound Healing Is Accelerated by a Lipid Mixture Representing Major Lipid Components of Chamaecyparis obtusa Plant Extract. J Invest Dermatol. 2018;138(5):1176-86.

55. Khezri K, Farahpour MR, Mounesi Rad S. Accelerated infected wound healing by topical application of encapsulated Rosemary essential oil into nanostructured lipid carriers. Artif Cells Nanomed Biotechnol. 2019;47(1):980-8.

56. Mazutti da Silva SM, Rezende Costa CR, Martins Gelfuso G, Silva Guerra EN, de Medeiros Nobrega YK, Gomes SM, et al. Wound Healing Effect of Essential Oil Extracted from Eugenia dysenterica DC (Myrtaceae) Leaves. Molecules. 2018;24(1).

57. Prommaban A, Utama-Ang N, Chaikitwattana A, Uthaipibull C, Srichairatanakool S. Linoleic acidrich guava seed oil: Safety and bioactivity. Phytother Res. 2019;33(10):2749-64.

58. Franz MG, Kuhn MA, Nguyen K, Wang X, Ko F, Wright TE, et al. Transforming growth factor beta(2) lowers the incidence of incisional hernias. J Surg Res. 2001;97(2):109-16.

59. Dubay DA, Wang X, Kuhn MA, Robson MC, Franz MG. The prevention of incisional hernia formation using a delayed-release polymer of basic fibroblast growth factor. Ann Surg. 2004;240(1):179-86.

60. Korenkov M, Yuecel N, Koebke J, Schierholz J, Morsczeck C, Tasci I, et al. Local administration of TGF-beta1 to reinforce the anterior abdominal wall in a rat model of incisional hernia. Hernia. 2005;9(3):252-8.

61. Franz MG. The biology of hernia formation. Surg Clin North Am. 2008;88(1):1-15, vii.

62. Henriksen NA. Systemic and local collagen turnover in hernia patients. Dan Med J. 2016;63(7).

63. Sharma A, Berger D. The current role of laparoscopic IPOM repair in abdominal wall reconstruction. Hernia. 2018;22(5):739-41.

64. Yang GPC. From intraperitoneal onlay mesh repair to preperitoneal onlay mesh repair. Asian J Endosc Surg. 2017;10(2):119-27. 



\section{Chapter 10}

Summary 
Chapter 10 
Tissue healing is a biological response of traumatized tissues to restore the structural origination and normal function after surgical injury. Failure of the restore process in abdominal fascial incision can result in incisional hernia (IH) formation while overrestored fibrous tissues can form intraperitoneal adhesions. IH and intraperitoneal adhesions are two of the most common complications following laparotomies. This thesis aims to investigate the normal healing process of abdominal fascia overtime and the efficacy of antiadhesive barriers that could prevent the formation of intraperitonal adhesions.

Animal experiment plays an indispensable role in translational research, although alternative models have been developed. High quality of paper reporting maximizing details of animal experiments can increase the reproducibility of the study and decrease the number of animal use. Chapter 2 presents the effect of ARRIVE-2010 and ARRIVE-2020 ((Animal Research: Reporting of In Vivo Experiments) guidelines on improving the reporting quality of articles using IH animal models repaired with meshes. Compared to articles published in 5 years before the publication of ARRIVE2010 guidelines (pre-ARRIVE), articles published in the latest 5 years until the update of ARRIVE-2020 guidelines (post-ARRIVE) were found with significantly higher mean numbers of fully reported (sub)items listed in ARRIVE. Compared with preARRIVE articles for per (sub)item, post-ARRIVE articles were significantly different in a few (sub)items, mostly reported with improvements while few reported worse post-ARRIVE. A large number of (sub)items remained unreported or inadequately reported post-ARRIVE. This was also found in articles published in the latest year using ARRIVE-2020 as the scoring criteria. Therefore, ARRIVE-2010 guidelines may have a limited effect on improving the reporting quality of animal experiments. There is much room left for further improvement of reporting for animal experiments. Furthermore, the reporting quality of articles between pre-ARRIVE and post-ARRIVE were also compared for specific species (rats, rabbits, and pigs, respectively), suggesting unbalanced effects of ARRIVE guidelines on the improvement of reporting in animal studies.

Animal gender should be taken into account in the design of animal experiments. Chapter 3 demonstrates the difference between male and female rats regarding the intraperitoneal adhesion formation as well as normal abdominal wall healing rate. No gender-related difference of adhesion score and abdominal wall wound score were identified between male and female rats using an ischemic-button adhesion model. However, we found male rats seem to gain weight faster than females during the oneweek follow-up postsurgery.

Failure of fascial healing in abdominal incision can form IH, while over-restored fibrous tissues can lead to intraperitoneal adhesions. Despite the importance of normal 
fascial healing, few studies have been focusing on fascial healing process. Chapter 4 displays the temporal changes of cellular population and gene expression levels in normal fascial healing. Animals received laparotomy closed with a single layer continuous suturing technique for fascia were allocated evenly to four different time points. Granulocytes macrophages were found with significant alteration during the normal healing process. Gene expression levels of markers for the two subtype macrophages (classically activated macrophages and alternatively activated macrophages) were significantly altered at three postoperative days. These results indicate that the inflammatory cells predominate from granulocytes to macrophages in early postoperative days. Furthermore, macrophage polarisation, a switched predomination from classically activated macrophages to alternatively activated macrophages, occurs in early fascial healing process.

Besides cells in the normal fascial healing, the temporal changes of lipid molecules in the healing process are important as potential treatment targets promoting fascial healing. Chapter 5 focuses on the investigation of changes of lipid spatial distribution related to inflammatory cells. Animals healed normally were sacrificed at seven different time points. Lipids were analyzed using matrix - assisted laser desorption/ionization combined with time - of - flight mass spectrometers (MALDITOF). The relationship between the intensity of any $m / z$ species and the healing time was analysed with linear regression using R. A significant difference was found in the analysis in a total of 35 mass to charge $(\mathrm{m} / \mathrm{z})$ values for negative ion mode and a total of $18 \mathrm{~m} / \mathrm{z}$ values for positive ion mode. Five different lipid species, belonging to phosphatidylcholines, phosphatidylethanolamines, and gangliosides, were found with correlation to inflammatory response or fibroblast growing postsurgery. These lipid species are potential biomarkers for predicting incisional hernia and could be the therapeutic targets preventing incisional hernia.

Chapter 6 compares the antiadhesive efficacy of five novel antiadhesive barriers using an ischaemic button animal model. These physical barriers were placed intraperitoneally isolating internal organs and peritoneum from ischaemic buttons. One of these antiadhesive polymer barriers (A2 barrier) was found with significantly lower numbers of buttons involved with adhesions in comparison to others. However, no significant difference of adhesion score was found between these antiadhesive barriers, using Nair's score (quantity score) or Zühlke score (quality score). Therefore, the antiadhesive efficacy of A2 barrier needs further investigation. Chapter 7 evaluates the antiadhesive efficacy of a novel hyaluronic acid gel using the aforementioned ischaemic button model. Compared to the hyaluronic acid/carboxymethyl cellulose gel, the novel gel significantly reduced intraperitoneal adhesions only when measured with the number of involved organs. Results from pathophysiological analysis of the 
adhesion tissues together with ischaemic buttons indicate a prolonged activation of alternatively activated macrophages (M2-like phenotypes).

Antiadhesive barriers coated on meshes are commonly used to prevent tissue-to-mesh adhesion formation. A large number of previous animal experiments were used to test the antiadhesive efficacy of mesh coatings, lacking consistent results. Chapter 8 summarizes the antiadhesive performance of mesh coatings. A systematic review and meta-analysis was performed aiming to make comparisons between meshes with coatings and meshes without coatings. In our integrated results, polypropylene meshes coated with hyaluronic acid/carboxymethyl cellulose showed a significant reduction of intraperitoneal adhesion formation compared to polypropylene meshes. Heterogeneity was low for this integration. There was no significant reduction of adhesion formation for polyester meshes coated with collagen compared to polypropylene meshes. However, heterogeneity for this comparison was high while the source of the heterogeneity was complex. These findings indicate that hyaluronic acid/carboxymethyl cellulose as a mesh coating shows antiadhesive property in animal models. 

第10章 总结 
Chapter 10 
术后创伤组织的愈合是创伤组织恢复其原有结构和功能的正常生物学反应。就腹 部切口而言, 腹壁切口中肌腱膜层愈合不良会导致腹壁切口疝, 而腹腔内过多纤 维组织的形成及黏附会形成腹腔粘连, 进而引发腹腔粘连相关的并发症, 例如疼 痛, 肠梗阻，女性不孕，以及给再次手术时带来困难甚至导致肠道损伤。本论文 旨在探讨术后早期腹壁腱膜的正常愈合过程以及评估新型抗粘连材料预防腹腔粘 连的功效。

虽然目前已发展了多种实验方法替代动物模型以减少实验动物的数量, 但是, 动 物实验在目前的生物医学研究中仍旧起着不可或缺的作用。最大限度的报告动物 实验的细节信息, 可以提高动物实验的可重复性, 进而可能减少实验动物的使用 数量。本论文第二章介绍了 ARRIVE2010（动物研究：体内实验的报告）指南对 动物实验类科学论文的报告质量的改善效果，侧重于评估使用疝补片修补腹壁疝 相关的动物实验。将 ARRIVE2010 指南发布之前 5 年内发表的论文与更新的 ARRIVE2020 发布之前 5 年内发布的论文进行比较, 以 ARRIVE2010 中的条目/子 条目的平均报告数量为评价标准, 我们发现后者明显改善, 有统计学差异。然而 , 在 ARRIVE2010 指南发布后的动物实验类论文中, 仍有许多根据 ARRIVE 指南应 该报告的条目/子条目未进行报告。由此，我们得出结论：ARRIVE2010 指南可能 改善了动物实验类论文的报告质量, 但是该类论文的报告质量仍有很大的改善空 间。同时, 我们也比较了在特定单独的物种中, 主要在大鼠, 兔子和猪中, ARRIVE2010 发布前组和 ARRIVE2010 发布后组相关论文的报告质量。结果显示, 以不同物种为模型的动物实验, ARRIVE2010 指南对其论文的报告质量的改善效 果是不均衡的。对最近一年内发表的论文以 ARRIVE2020 为标准进行评估后发现 ，同样的，许多应该报告的核心条目仍未被充分的报告。

在动物实验的设计及分析中, 动物性别作为一个很重要的独立变量需要考虑。第 三章比较了由雌性和雄性大鼠的性别差异导致的在腹腔粘连形成和腹壁正常愈合 方面的差异。其结果显示雌雄大鼠之间并没有性别导致的粘连得分以及腹壁愈合 得分的统计学差异。但是, 在为期一周的术后恢复中, 我们发现雄性大鼠的体重 增长明显快于雌性。

腹壁腱膜切口愈合失败会导致切口疝的形成。尽管腹壁腱膜愈合很重要, 但很少 有相关的研究针对术后早期腹壁腱膜的正常愈合。第四章研究了腹壁腱膜切口正 常愈合中细胞数量和基因表达水平随时间的变化。将实验动物分配给术后一周中 四个不同的时间点。经过正中切口的剖腹手术后, 将腹壁腱膜单层连续缝合, 在 各时间点收获腹壁腱膜愈合的标本。我们发现粒细胞和巨噬细胞在正常愈合过程 中有显著的改变。占主导地位的主要炎症细胞在术后早期的愈合中从粒细胞过渡 到了巨噬细胞。在术后第三天，巨噬细胞亚型（“经典激活型巨噬细胞” 和 “非 经典激活型巨噬细胞）的标志物在基因表达水平上发生了显著的变化。因此，巨 噬细胞在腹壁腱膜愈合的早期就发生了极化，从经典激活型巨噬细胞占主导地位 切换到了由非经典激活型巨噬细胞占主导地位。 
除了涉及正常腹壁腱膜愈合的细胞和基因外，脂类分子在愈合过程中的变化也值 得关注。之前的研究显示脂类化合物可以成为促进腹壁腱膜愈合的治疗制剂。第 五章着重研究了与炎症细胞相关的脂类分子随时间变化的空间分布。在术后一周 中七个不同的时间点获取腹壁腱膜正常愈合的动物标本, 使用基质辅助激光解吸 /电离结合飞行时间质谱仪 (MALDI/TOF) 进行脂类分析。以 $\mathrm{R}$ 语言构建了线性回 归模型以研究质荷比的信号强度和愈合时间的关联。在负离子模式下总共有 35 个不同质荷比的粒子被发现在线性回归中存在显着差异，而在正离子模式下有 18 个不同质荷比的粒子存在这种显著性差异。五种隶属于磷脂酰胆碱，类磷脂 酰乙醇胺及神经节苷酯的脂类物质与腹壁腱膜急性损伤后的炎症反应和纤维母细 胞生长显著相关。这些脂质可能成为潜在的腹壁腱膜愈合的生物标志物, 并可能 成为预防切口疝的治疗靶标。

使用材料屏障抗腹腔粘连是目前主流的抗腹腔粘连的策略, 然而理想的此类材料 仍在搜寻中。第六章在 “缺血性纽扣” 腹腔粘连的动物模型中评估了五种新型材 料的抗粘连功效。将这些抗粘连材料于手术中放置于 “缺血性纽扣” 表面和内脏 器官表面之间, 隔离其相互粘连。与其他的抗粘连材料相比, 我们发现其中一种 高分子聚合物抗粘连材料（A2）明显降低了内脏器官与 “缺血性纽扣”之间粘连 形成的数量。但是, 使用 Nair 评分系统（侧重于评估粘连产生的数量）或 Zühlke 评分系统（侧重于评估粘连产生的质量），我们在参与评估的抗粘连材 料之间没有发现有统计学意义的显著性差异。因此, A2 抗粘连材料的抗粘连性 能需要进一步实验进行研究和评估。第七章评估了一种新型抗腹腔粘连的透明质 酸凝胶, 其结果是其抗粘连效果也仅仅降低了 “缺血性纽扣” 的粘连数量。以 Nair 或 Zühlke 粘连评分系统作为评价标准，各种参与评估的凝胶的抗粘连效果 相互之间并无显著性差异。

在腹壁疝补片涂上抗粘连表层材料可用于防止粘附于疝补片的粘连形成。虽然许 多动物实验测试了疝补片表面涂层材料的抗粘连效果, 但是在动物实验中这些结 果并不一致而且不易相互比较。第八章归纳了有关评估疝补片涂层材料的抗粘连 功能的动物实验。我们进行了系统综述和荟萃分析, 对有涂层的和无涂层的疝补 片进行了比较。与聚丙烯疝补片相比, 涂有透明质酸/羧甲基纤维素的聚丙烯疝 补片显著地抑制了粘连的形成。其中各荟萃分析中的动物实验的异质性很低, 结 果相对可信。与聚丙烯疝补片相比, 涂有胶原蛋白的聚酯疝补片没有显著性的减 少粘连形成。但是, 在此比较中, 各荟萃分析中的动物实验之间异质性很高, 异 质性的来源复杂, 不易辨别。由此可以得到的结论是, 透明质酸/羧甲基纤维素 在动物模型中作为网片涂层显示出良好的抗粘连性能。 


\title{
Chapter 11
}

\author{
Valorisation
}


Chapter 11 
Translational research has solidified its critical role in current health, medicine and life science thanks to the overriding goal to benefit human health, providing information on health or disease from various insights. Information from this type of research helps to improve strategies for prevention, prediction, diagnosis, and treatment of disease. More than 200 years after the first elective laparotomy, healing-related complications post laparotomies, such as incisional hernia (IH) and intraperitoneal adhesion formation, still generate discomfort experienced by patients as well as high costs for society. All studies performed in this thesis are intended to improve the knowledge of regeneration processes that could prevent the formation of intraperitoneal adhesions or promote normal healing of abdominal fascia. This chapter describes possible clinical relevance, as well as related individual/social benefits, using the results in the present thesis.

\section{Economic and social relevance}

Around 100,000 laparotomies are performed annually in the Netherlands. ${ }^{1}$ One common complication following laparotomy is the formation of intraperitoneal adhesions, ranging from $60 \%$ to $80 \%$ post laparotomy for both short-term and longterm follow-up. ${ }^{2}$ Intraperitoneal adhesions can lead to chronic abdominal pain, female infertility, and small bowel obstruction. Furthermore, adhesiolysis, the procedure of releasing the adhesions, at a repeat surgery links to a higher risk of full-thickness bowel defects, sepsis, longer operative time, delayed hospital stay, more readmissions, and increased costs, compared to non-adhesiolysis. ${ }^{3}$ The use of antiadhesive products have beneficial effects on patients and is cost-effective. ${ }^{4,5}$ For example, if an antiadhesive product costs 130 euros and has an antiadhesive efficacy of $25 \%$ in one year, more than 40 million euros over ten years postsurgery could be saved using this product in the UK. ${ }^{6}$ Therefore, the evaluations of antiadhesive efficacy for the newly developed barriers in this thesis potentially bring economic benefits to society, besides from the beneficial effects on the quality of life of the patients.

$\mathrm{IH}$ is another common complication following laparotomy, leading to discomfort, bowel obstruction, incarceration and strangulation. ${ }^{7,8}$ Despite watchful waiting as a treatment strategy for a large number of IH patients, IH repairs are declared yearly on an average of 4,200 patients by the Dutch Hernia Authority in The Netherlands. Compared to the use of only sutures for IH repair, the use of synthetic meshes for the IH repair can significantly reduce IH recurrence $(23.8 \%$ vs. $7.7 \%$ recurrence for suture vs. mesh repair). ${ }^{9}$ Moreover, prophylactic mesh augmentation in primary fascial closure for patients with high-risk of IH occurrence can effectively decrease IH formation. ${ }^{10}$ However, mesh placement for IH repair or prevention facilitates possible mesh-related complications, including infection, seroma, hematoma, and fistulas. 
Additionally, the mean age for incisional hernia patients is in the fifth decade of life. ${ }^{11}$ It is a working-age for most patients and subsequently can cause much social burden. Furthermore, obesity is one of the independent risk factors for the formation of $\mathrm{IH}$. Cases of obesity are growing in The Netherlands, the number of IH patients could increase subsequently. This thesis revealing the cell and molecular events in the normal fascial healing overtime could provide knowledge to develop preventive strategies for IH. Consequently, it can have beneficial effects on preventing postoperative complications as well as saving costs for society.

\section{Scientific relevance}

IH represents a defect of continuity of abdominal fascia, caused by the failed healing of abdominal fascia. Normal fascial healing plays a pivotal role in the prevention of IH formation. Despite the fact that virtually all tissues have similar healing stages, various types of cells, molecules, and pathways during the healing process differ in specific tissues. Few studies concern the normal healing process of abdominal fascia, especially in the early postoperative period. To fill this gap, this thesis has assessed the involved cells and related molecules in the normal fascial healing process.

There is concern about the translation of preclinical results to humans. However, van den Hil et al. have shown that the results could be comparable between rats and humans in IH. ${ }^{12}$ Furthermore, Occleston et al. have clearly described the molecular comparability in wound healing between rats and humans. ${ }^{13}$ Therefore, the results regarding fascial healing in this thesis could be translated to humans despite further human trials are encouraged.

Compared to IH repair only using sutures, mesh augmentation correcting $\mathrm{IH}$ reduces the recurrence of IH significantly. Placement of prophylactic meshes in high-risk patients (for example, patients with obesity or abdominal aortic aneurysm) could provide augmentation to the integrity of abdominal wall and help to prevent $\mathrm{IH}$ effectively. Intraperitoneal onlay mesh placement has an important role in laparoscopic hernia repair in clinical practice. One problem after the mesh placement is the formation of adhesions to the mesh. Antiadhesive coatings on the mesh are one of the major strategies to prevent this adhesion formation. This thesis has systematically summarized the antiadhesive efficacy of different types of coatings in animal models, which could facilitate the development of mesh coatings in the future.

Implantation of physical barriers separating the contact between tissue layers is the main strategy to prevent intraperitoneal adhesion formation. Commercially available 
forms of these physical barriers can be films, gels, or solutions. The search for ideal barriers is ongoing because of insufficient antiadhesive efficacy, interruption of normal healing of adjacent organs, or inconvenience of application for previous products. One aim of this thesis is to evaluate the anti-adhesive efficacy of alternative newly developed barriers.

Animal models contribute scientific benefits to humans because products first tested successfully in animal models could reduce the failure opportunities in humans. Maximizing the reported information of animal experiments could reduce the use of animals and increase the reproductively. ARRIVE (Animal Research: Reporting of In Vivo Experiments) guidelines have been published with a purpose to improve reporting quality of animal experiments. This thesis has evaluated the effect of ARRIVE guidelines on reportied quality of previously published articles, trying to encourage awareness of maximizing reported information and subsequently reducing the animal use.

Gender-related difference of animals, as a basic variable in animal experiments, should be taken into account in the design of animal experiments. This thesis has compared the baseline difference of the quantity and quality score of adhesions, as well as the normal fascial healing rate, between male and female rats using an ischaemic- button adhesion model. Evaluating the baseline gender difference of an animal model could provide evidence-based data for the sex consideration in further design in animal testing. Moreover, it could provide data to calculate sample size of further animal experiments, complying with the refinement principle for animal use.

\section{Target groups}

Intraperitoneal adhesions and $\mathrm{IH}$ are two of the most common complications after laparotomies, surgeons performing any abdominal surgery are therefore the target groups for this thesis. Gastro-Intestinal surgeons are especially suitable target group. This is because small bowel-obstructions are mainly caused by intraperitoneal adhesions and consequent adhesiolysis releasing the adhesions can lead to iatrogenic full-thickness bowel injury in $10.5 \%$ adhesiolysis patients. ${ }^{3}$ Moreover, the Dutch Association of Surgeons suggested that "incisional hernias may only be operated/supervised by a certified Gastro-Intestinal surgeon" from May 2019. All patients undergoing laparotomies can potentially benefit from this thesis, because this thesis focuses on promoting normal fascial healing and preventing intraperitoneal adhesion formation after laparotomies. Additionally, the findings in this thesis have beneficial effects on academic researchers who are interested in the prevention of 
intraperitoneal adhesions and the promotion of abdominal fascial healing. Both researchers using animal models and animal caretakers, encouraged to be aware of the gaps of current animal experiment reporting, are also target groups.

\section{Innovation and implementation}

Despite much progress has been achieved regarding the prevention of intraperitoneal adhesions and $\mathrm{IH}$, for instance, the application of preventive materials and strategies, perfect ones lack currently. Knowledge of the underlying pathophysiology of these two complications on cellular and molecular levels, as well as novel effective materials, is needed. The findings in Chapter $\mathbf{4}$ and $\mathbf{5}$ could help reveal temporal changes in normal fascial healing in the early postoperative days. Evaluation of novel materials preventing the over healing of intra-abdominal adhesions in Chapter 6 and 7 could promote the development of novel antiadhesive barriers. Systematic comparisons of anti-adhesive mesh coatings in chapter 8 could benefit the searching for more effective mesh coatings. Additionally, evaluation of reporting quality based on ARRIVE guidelines in Chapter 2 could increase the awareness of maximizing animal reporting information and subsequently promote the reproductivity of animal experiments, benefiting further animal experiments. Sex-related difference of baseline performance between male and female animals in Chapter 3 could provide evidence-based data for gender consideration in the design of further animal experiments. 


\section{References}

1. Prismant. Utrecht, the Netherlands: National Medical Registration. 2002.

2. Stommel MWJ, Ten Broek RPG, Strik C, Slooter GD, Verhoef C, Grunhagen DJ, et al. Multicenter Observational Study of Adhesion Formation After Open-and Laparoscopic Surgery for Colorectal Cancer. Ann Surg. 2018;267(4):743-8.

3. ten Broek RP, Strik C, Issa Y, Bleichrodt RP, van Goor H. Adhesiolysis-related morbidity in abdominal surgery. Ann Surg. 2013;258(1):98-106.

4. ten Broek RP, Bakkum EA, Laarhoven CJ, van Goor H. Epidemiology and Prevention of Postsurgical Adhesions Revisited. Ann Surg. 2016;263(1):12-9.

5. Wilson MS. Practicalities and costs of adhesions. Colorectal Dis. 2007;9 Suppl 2:60-5.

6. Wegdam JA, de Vries Reilingh TS, Nienhuijs SW, Simons MP. Abdominal wall hernia surgery in The Netherlands: a national survey. Hernia. 2020;24(3):601-11.

7. Petersson J, Koedam TW, Bonjer HJ, Andersson J, Angenete E, Bock D, et al. Bowel Obstruction and Ventral Hernia After Laparoscopic Versus Open Surgery for Rectal Cancer in A Randomized Trial (COLOR II). Ann Surg. 2019;269(1):53-7.

8. Azin A, Hirpara D, Jackson T, Okrainec A, Elnahas A, Chadi SA, et al. Emergency laparoscopic and open repair of incarcerated ventral hernias: a multi-institutional comparative analysis with coarsened exact matching. Surg Endosc. 2019;33(9):2812-20.

9. Mathes T, Walgenbach M, Siegel R. Suture Versus Mesh Repair in Primary and Incisional Ventral Hernias: A Systematic Review and Meta-Analysis. World J Surg. 2016;40(4):826-35.

10. Muysoms FE, Antoniou SA, Bury K, Campanelli G, Conze J, Cuccurullo D, et al. European Hernia Society guidelines on the closure of abdominal wall incisions. Hernia. 2015;19(1):1-24.

11. Burger JW, Luijendijk RW, Hop WC, Halm JA, Verdaasdonk EG, Jeekel J. Long-term follow-up of a randomized controlled trial of suture versus mesh repair of incisional hernia. Ann Surg. 2004;240(4):578-83; discussion 83-5.

12. van den Hil LCL, Vogels RRM, van Barneveld KWY, Gijbels MJJ, Peutz-Kootstra CJ, Cleutjens JPM, et al. Comparability of histological outcomes in rats and humans in a hernia model. J Surg Res. 2018;229:271-6.

13. Occleston NL, Metcalfe AD, Boanas A, Burgoyne NJ, Nield K, O'Kane S, et al. Therapeutic improvement of scarring: mechanisms of scarless and scar-forming healing and approaches to the discovery of new treatments. Dermatol Res Pract. 2010;2010. 

Part V

Appendix 

Acknowledgements 



\section{Acknowledgements}

I am grateful to my Assessment Committee, prof. dr. L.P.S Stassen, prof. dr. R. Van der Hulst, prof. dr. J. Lange, prof. dr. L. Moroni, and dr. R.P.G. ten Broek. Thank you very much for your effort and time to evaluate this thesis critically.

Lots of grateful moments accompany the completion of this thesis. I truly appreciate all the help and support I have received leading to this thesis, from my supervision team, my colleagues, my friends, and my family. I could not finish it without your help and encouragement.

First of all, I would like to show my sincere gratitude to prof. Nicole D. Bouvy. Dear Nicole, I could not even believe the first time that a professor with profound knowledge can be so kind to a student, especially from a quite different cultural country until I contacted you. It is very impressive for me that you shared your lunch to me during a tight break of surgeries you performed when I arrived at Maastricht University the first time. Under your guidance and arrangements, I felt comfortable to join your team without a too hard beginning. Every week you enquired all the problems I met in both research and life and helped me solve these problems in time. Every time after I met you, difficult $\mathrm{PhD}$ time seems to become easier for me. Even during the pandemic period, I still received your support for my academic progressing. I learnt a lot from you, not only about the academic knowledge but also the respectful attitude to every communicator you faced. There are lots of beautiful moments you left in my mind, driving me and my family to the Fun Valley, attending a conference together, playing underground laser games together, joining us to taste foods and drinks at restaurants, providing Asian food for us at your home, making nice cucumber tea yourself for us, birthday greetings to me, and so on. I am so lucky being one member of your team. Thank you from the bottom of my heart, Nicole!

Dear Kaatje, You provided me with professional suggestions for my research projects and always helped me out when I lost in the researches. You are really helpful, especially for the finalisation of this thesis! I learnt much from your professional manner in academic researches. Please accept my best thanks!

Dear prof. Lorenzo, thank you very much for providing me with opportunities to conduct animal experiments, as well as to continue the histology. I gained a lot of experience from this, which could be very helpful for my future career. You are really great!

Dear prof. Ron, I am most grateful for your support to push the progress of the project. Your profound knowledge and strict scholarly working attitude in academic research 
while accessible and kind in common life are so impressive to me. Your enthusiasm during a meeting or discussion is very attractive to me.

Dear prof. Steven, I was glad to hear your comments and inspiring questions on the weekly research meeting and learnt much from you. It was lucky for me to be allocated to your team during a labout. You took a big stone for a long distance to complete a task for our whole team. It is just like that you supported the whole research department with the wonderful academic environment.

Dear Benjamin, I truly appreciate that you helped me out during the most difficult period of my PhD. You are so nice! You spent much time and energy on coding to save the project. Each time when we sit together for the project, I was triggered by your nice help. Without you, the project could not have been done. Thanks again for your amazing work and academic guidance!

Dear Marion, since the first time I contacted you, you were always enthusiastic to give a response. You helped me much for the histological evaluation of my samples. You frankly speaking style with a warm voice is very nice. I am very thankful that you contributed a lot to the project!

Dear CPV staff, Richard, Saskia, Rik, Clarice, Petra, Huub, Inger, and Rachelle, I really enjoy these days spent with you during animal experiments. I still remember the inspired words for animal experiments, 'nothing is impossible until it is done'. Truly thank you all, you guys!

Dear Bjorn, it is very nice of you to help me with the complex statistics. You are very professional and have many nice suggestions. Thanks again!

Dear dr. Pieter, it is very kind that you trained me on performing orthopaedic surgeries in rabbits. Without your guidance and hand-to-hand teaching, I could not overcome the surgical skill difficulties. Many thanks!

Dear Sander, it is very kind of you to talk a lot with me. Your research questions inspired me a lot. I really appreciate it!

Dear Mo and Bas, I received generous help from you! You booked the computer and laptop for me and kept it updated. You left your own work and accompanied me to pick up opium medications. Whenever I had a request, you were always patient to help out. You are always the 'first aid' for me in the lab. Deeply thank you! 
Dear Filipa, thanks a lot for teaching me all the histological skills with much patience to explain all details. You arranged everything of the histology well me. You are a nice teacher and really helpful!

Dear Marloes and David Koper, I appreciate your help in animal experiments! Together with you, I felt much better during those surgeries. You made my day!

Dear Elwin, the aboard journey was a bit hard for me in the first months. Fortunately, you guided me a lot and did much for me, ranging from getting the university access, introducing office and labs, taking me to a course, inviting me to your home, driving me to a climbing game. Thank you, my friend!

Dear Leontine, thanks for sharing your projects before you left, as well as the cakes and the book. I was not aware of the importance of these projects at that moment, but I realised that later how precious they are.

Dear Audrey, I always followed in your footsteps on research in the first year of my $\mathrm{PhD}$. I disturbed you many times, but you were always very kind to help me even when you were very busy. Many thanks!

Dear Jacqueline, you were the girl sitting beside me in the office. I got much help from you. I still remember that you accompanied me to the mail service and closed the envelope in a special way. Thank you!

Dear Anne-Claire, thank you for your effort contributing to our project even you were very busy dealing with clinical affairs. Your suggestions for our project always hit the point!

Dear Sebastiaan, it is really good to meet you, my friend! You really helped me a lot in both research and life. Together for animal experiments, workday lunch, weekly meeting, conferences, and parties, I felt very comfortable to stay with you!

Dear Anne, it is very happy to do animal experiments together with you. It is very impressive that you organised everything well even for those experimental procedures you never experienced, well-done! Your kindness, humour, and excellent communication skills always saved my day! A large number of colourful moments together with you are left in my heart.

Dear Aurelia, it is nice that I received your proactive help for my project. You explained all details patiently for the animal reviews. Thanks! 
Dear Maurits-Jan, you made a lot of effort to our articles, thank you! You are active, working hard, and excellent at academic English writing. I wish you success in your future career!

家平哥, 初来荷兰时一无所知, 遇到很多困惑。你不愿其烦, 有问必答, 答解 时又务求详尽, 贴合我的实际情形, 十分感谢! 也正是有你的帮助, 来这后住 的舒适, 还少了不少搬家的烦心事儿。也感谢你和你夫人的盛情款待, 让我感 受到了真同胞的温暖。今后的日子还得多多麻烦你。但凡有需要我的地方, 开口 便是。

王萍, 开美, 很荣幸认识你们, 生活上得到你们不少的帮助, 非常感谢! 铭烨 非常开心认识你们, 结交了开美这个朋友, 留下了很多美好的回忆。疫情过后, 我们还是一如往常, 多多走动。

高叶, 杨奇, 很开心认识你们, 也很开心可以就小孩学校的事情相互交流。经常 能碰见你们, 来日方长, 继续交流。

俊芳, 初到科室时受到你莫大的照顾, 解决了不少实际难题。也让我有了学习的 榜样, 多了一份信心完成攻博。也感谢你的款待, 离开荷兰时还送了我东西。如 今你已定居武汉，相信来日必有再相见。

新伟, 陈琳, 邓敏, 建花, 秀香, 同一屋檐下的同事朋友, 感谢你们课题上的支 持, 生活上的帮助。希望将来继续多多交流, 相互团结, 互帮互助, 成功完成 课题, 同时留下美好的生活回忆。

最后, 感谢我的家庭成员, 感谢你们付出了代价, 让我重返学校得以提升。同时 不远万里, 前来陪伴。 
List of publications 



\section{List of publications}

1. Liu H, Gielen M-JJCAM, Bosmans JWAM, Winkens B, Bouvy ND. Inadequate awareness of adherence to ARRIVE guidelines, regarding reporting quality of hernia models repaired with meshes: a systematic review. Hernia. 2021.

2. Mommers EH, Hong L, Jongen A, Bouvy ND. Baseline performance of the ischaemic button model for induction of adhesions in laboratory rats. Lab Anim. 2019;53(1):63-71.

3. Liu H, Jongen ACHM, Chang X, Melenhorst J, Lenaerts K, Gijbels MJ, et al. Temporal changes in cell populations and gene expression during abdominal wall fascial healing. Molecular biology.(Submitted)

4. Liu H, Cao J, Balluff B, Jongen ACHM, Gijbels MJ, Melenhorst J, et al. Abdominal fascial healing in the first postoperative week: a MALDI-TOF mass spectrometry imaging to investigate lipids in rats Clinical mass spectrometry.(In revision)

5. van Steensel S, Liu H, Mommers EHH, Lenaerts K, Bouvy ND. Comparing Five New Polymer Barriers for the Prevention of Intra-abdominal Adhesions in a Rat Model. J Surg Res. 2019;243:453-9.

6. van Steensel S, Liu H, Vercoulen TF, Hadfoune M, Breukink SO, Stassen LP, et al. Prevention of intra-abdominal adhesions by a hyaluronic acid gel; an experimental study in rats. J Biomater Appl. 2020:885328220954188.

7. Liu H, van Steensel S, Gielen M, Vercoulen T, Melenhorst J, Winkens B, et al. Comparison of coated meshes for intraperitoneal placement in animal studies: a systematic review and meta-analysis. Hernia. 2019. 

Curriculum Vitae 



\section{Curriculum Vitae}

Hong Liu was born on $17^{\text {th }}$ of February 1981 in Xiangyang City, China. After graduating from a high school in Xiangyang, Hong started his studies at Zhejiang University. After spending five years there, he got his bachelor degree with a major in clinical medicine. In the last half-year of the five years, he witnessed the first time how surgeries work at a hospital and how animal researches go at the lab. He began his clinical internship at the Second Affiliated Hospital (Zhejiang University School of Medicine) and Ningbo First Hospital. At the end of the second year of the internship, he finished his Master degree in clinical medicine at Zhejiang University and was certificated with a resident in surgery.

After graduated from Zhejiang University, he commenced his work at general surgery department at the Third Hospital in Wuhan City, where he was awarded 'Outstanding Young Doctors' in 2009. He passed the Doctor's License in 2013. In 2016, he was awarded a scholarship under the State Scholarship Fund from China Scholarship Council. With this scholarship, together with the guidance and support of prof. dr. Nicole Bouvy, he set out his research in the Netherlands. He started his full- time PhD candidate under the supervision of prof. dr. Nicole Bouvy and dr. Kaatje Lenaerts, leading to this thesis. In 2020, he will continue his research work at the MERLN department at Maastricht University with combined support of prof. dr. Nicole Bouvy and prof. Lorenzo Moroni. 
